University of Louisville

ThinkIR: The University of Louisville's Institutional Repository

Electronic Theses and Dissertations

8-2002

\title{
Structure-function studies of the vaccinia virus complement control protein.
}

Scott Alan Smith

University of Louisville

Follow this and additional works at: https://ir.library.louisville.edu/etd

\section{Recommended Citation}

Smith, Scott Alan, "Structure-function studies of the vaccinia virus complement control protein." (2002). Electronic Theses and Dissertations. Paper 1347.

https://doi.org/10.18297/etd/1347

This Doctoral Dissertation is brought to you for free and open access by ThinkIR: The University of Louisville's Institutional Repository. It has been accepted for inclusion in Electronic Theses and Dissertations by an authorized administrator of ThinkIR: The University of Louisville's Institutional Repository. This title appears here courtesy of the author, who has retained all other copyrights. For more information, please contact thinkir@louisville.edu. 


\title{
STRUCTURE-FUNCTION STUDIES OF THE VACCINIA VIRUS COMPLEMENT CONTROL PROTEIN
}

\author{
By \\ Scott Alan Smith \\ A.S. Community College of Allegheny County, 1995 \\ B.S. California University of Pennsylvania, 1997
}

\begin{abstract}
A Dissertation
Submitted to the Faculty of the

Graduate School of the University of Louisville in Partial Fulfillment of the Requirements

for the Degree of

Doctor of Philosophy

Department of Microbiology and Immunology

University of Louisville

Louisville, Kentucky
\end{abstract}

August 2002 


\title{
STUCTURE-FUNCTION STUDIES OF THE VACCINIA VIRUS COMPLEMENT CONTROL PROTEIN
}

\author{
By
}

Scott Alan Smith

A.S. Community College of Allegheny County, 1995

B.S. California University of Pennsylvania, 1997

A Dissertation Approved on

March 27, 2002

by the following Dissertation Committee:

Dissertation Director 


\section{DEDICATION}

I dedicate the work presented in this dissertation to my mother, Toni Smith, who not only gave me quality genes, but also the nurturing and knowledge to exploit them. She has always encouraged me to explore my curiosity, and has taught me to be determined and persistent with my dreams. 


\section{ACKNOWLEDGEMENTS}

I would first like to thank my advisor, Dr. Girish Kotwal, for all his time, guidance, and endless diligence. I am extremely grateful for his kindness and all the effort he made in ensuring that my goals were achieved. I will never forget the many long nights we spent preparing manuscripts for submission, or the long discussions we have had. I am also indebted to my dissertation committee, Drs. David Justus, Larry Hunt, Jaydev Dholakia, and Robert Stout for their advise, guidance, and thorough review of my dissertation. In addition, I would like to express thanks to my fellow students and lab mates: Jaime Anderson, David Reynolds, Barry Billings, Purushottam Jha, Ming Yan Yang, Zhouning Zhang, and Kristen Keeling, for all their assistance and comradeship over the last four years.

Many collaborating labs also need to be acknowledged. I would first like to thank Dr. Paul Barlow and his group from the University of Edinburgh, Scotland, including: Nick Mullins, Krystyna Bromek, Alan Cooper, and John Parkinson for their work with the structure and stability of VCP. Next, I would like to thank Dr. Krishna Murthy and his group from the University of Alabama at Birmingham, including: Gunasekaran Krishnasamy, and Ken Judge, for their work on the crystal structure of VCP and preparation $\mathrm{C} 3 / \mathrm{C} 4$. In addition, thanks must be given to my friends and collaborators in South Africa, Drs. Shrihari Arjunwadkar, and David Pugh for their help with the Biacore experiments. 
Finally, and most importantly, I would like to thank the love of my life, Helen, for enduring many long years of only brief encounters and innumerable phone calls and letters. Thank you for your support, encouragement, and never-ending faith in my ability. You are, and will always be my driving and inspirational force. I would also thank my family and friends for their patience while I am so distant. 


\begin{abstract}
Structure-Function Studies of the Vaccinia Virus Complement Control Protein Scott Alan Smith
\end{abstract}

March 27, 2002

The vaccinia virus complement control protein (VCP) is involved in the modulation of the host inflammatory response during vaccinia virus infection. It possesses the ability to inhibit both classical and alternative pathways of complement activation, as well as bind to heparin or heparan sulfate proteoglycans, making it a unique multifunctional protein with therapeutic potential. VCP is able to block complement activity through its ability to bind $\mathrm{C} 3 \mathrm{~b}$ and $\mathrm{C} 4 \mathrm{~b}$. Other very novel activities arise from VCP's ability to bind heparin and heparan sulfate proteoglycans, allowing the protein to attach itself to cell surfaces. The goal of this research was to fully characterize the structure and various functions of VCP.

The structural basis for VCP's ability to bind heparin was investigated using heparin affinity chromatography, surface plasmon resonance analysis, and homology modeling with well-characterized heparin binding proteins. VCP was found to possess two regions involved in heparin binding, one involved in weak binding located at the junction of complement control protein (CCP) modules 1 and 2, and a second involved in strong binding located at the extreme C-terminal tip of the protein. Additional functions, involved in blocking molecular interactions with cells, were also identified and determined to be a result of VCP's ability to bind heparin. 
The structural basis for VCP's ability to bind complement was investigated using the hemolysis assay, surface plasmon resonance analysis, and homology modeling with well-mapped complement regulatory proteins. VCP was found to be the smallest functional unit able to bind and cause factor I cleavage of $\mathrm{C} 3 \mathrm{~b} / \mathrm{C} 4 \mathrm{~b}$, thus inhibiting complement activity. VCP was also shown to possess the ability to simultaneously bind and inhibit complement activation, using its entire exposed surface, while remaining attached to heparin by its C-terminal heparin binding site.

The structure of VCP and its structural and functional stability were determined using X-ray crystallography, NMR, DSC, and the hemolysis assay. VCP was found to be an elongated filamentous protein, with defined CCP modular regions with limited intermodular interfacing, existing as a monomer in solution. VCP was found to possess the ability to structurally withstand temperatures in excess of $90^{\circ} \mathrm{C}$. In addition, VCP was able to retain functional integrity following exposure to many adverse physical conditions, which would result in irreversible denaturation of most other proteins.

Lastly, the in vivo pharmacokinetics of VCP was investigated in rats. VCP was injected as a bolus at different routes and using various concentrations. Continuous and intermittent intravenous injection strategies were also investigated. In all cases, the serum $\mathrm{CH} 50$ was monitored over time to determine the half-life of VCP activity in the serum. Traces of intact VCP was detected in urine by SDS-PAGE analysis, giving clues to its excretion and elimination from the body.

This research has provided greater understanding of the architectural features of a protein that contribute to heparin and complement binding. In addition, this research may prove important for the future use of VCP as a novel immunomodulating agent, as well as 
future protein-engineering efforts to design a better treatment for many complement mediated diseases, such as: Alzheimer's disease, central nervous system (CNS) injury, systemic lupus erythematosus, and xenograft transplant rejections. 
TABLE OF CONTENTS

PAGE

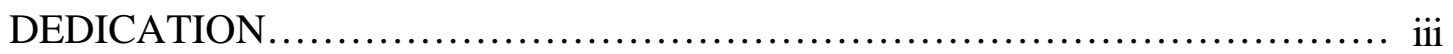

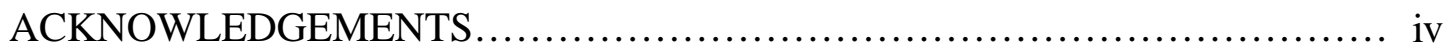

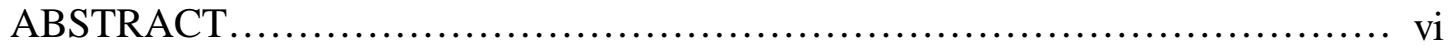

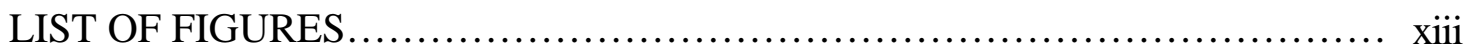

LIST OF ABBREVIATIONS........................................... xvii

\section{CHAPTER}

1. POXVIRUSES: VACCINIA VIRUS

Introduction.................................................. 1

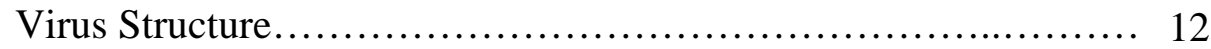

Genome Organization........................................ 16

Virus Life Cycle................................................. 20

Poxvirus Immunomodulation................................... 23

Complement Regulatory Proteins......................... 27

Cytokine Homologues...................................... 27

Cytokine Receptor Homologues (Viroceptors)............... 28

Chemokine Homologues.................................. 31

Chemokine Inhibitors.................................. 31

Serine Protease Inhibitors................................ 32

Vaccinia Virus Complement Control Protein (VCP)............... 35

\section{EXPRESSION, PURIFICATION, AND CHARACTERIZATION} OF VCP

VCP Produced from Natural VV Infection of Mammalian

Cell Culture................................................. 37

Pichia pastoris Yeast Expression System........................... 39

Growth and Affinity Purification................................ 44

BMGY -versus- BMMY ............................... 44

Affinity Purification and Endotoxin Removal................. 45

Assays and Characterization.................................... 54

Hemolysis Assay and Activity Units........................ 54

Protein Estimation Assay.................................... 57 
VCP Production for NMR, X-Ray Crystallography, and

Antibodies............................................... 58

Labeling for NMR................................ 58

Large Scale Purification for X-Ray Crystallography......... 61

Anti-VCP Production, Purification, and

Characterization......................................... 61

\section{HEPARIN BINDING ACTIVITY OF VCP}

Introduction to Heparin Binding................................ 67

Materials and Methods............................................ 73

Flow Microfluorimetric Analysis.......................... 73

Heparin Binding Ability................................. 74

Sequencing of the VCP Homologue in MPV.............. 75

Biacore Instrumentation............................... 75

Tri-peptide Studies................................... 78

Model of VCP.......................................... 79

X-Ray Crystallography................................ 80

Results................................................... 81

Specific Antibody Blocking to HUVECs.................. 81

Heparin Binding of rVCP and rVCP Fragments............ 86

Conserved K/R-X-K/R Sites.............................. 91

Biacore Heparin Binding................................. 96

Tri-peptide Studies .................................... 96

Dextran Sulfate Affinity Chromatography.................. 99

NMR Modeled Structure................................. 110

Electrostatic Charge Distribution......................... 110

X-Ray Crystal Structure Homology..................... 110

Discussion.................................................. 118

\section{COMPLEMENT BINDING ACTIVITY OF VCP}

Introduction to Complement Binding........................... 125

Materials and Methods............................................ 134

Hemolysis Assay........................................ 134

Preparation of rVCP and rVCP Truncations............... 134

Complement Component Purification..................... 135

Primary and Secondary Antibody Binding Analysis.......... 138

Simultaneous Heparin and Complement Binding............ 139

X-Ray Crystallography................................. 139

Results................................................... 140

Hemolysis Assay of Various Animal Sera.................. 140

Hemolysis Assay of rVCP Constructs.................... 140

Biacore Complement Binding.......................... 147 
Simultaneous Heparin and Complement Binding............ 154

X-Ray Crystal Structure Homology........................ 161

Discussion

\section{VCP STRUCTURE AND STABILITY}

Introduction to CCP Structure ................................ 174

Materials and Methods......................................... 178

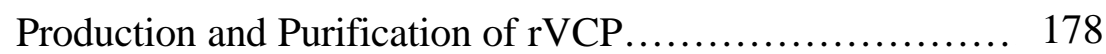

X-Ray Crystal Structure of rVCP....................... 178

Shelf-life............................................... 178

Freeze-thawing Sensitivity............................ 178

Temperature Sensitivity.............................. 179

pH Sensitivity........................................ 179

Hemolysis Assay....................................... 179

Gel Filtration.......................................... 179

Nuclear Magnetic Resonance (NMR)................... 180

Results

Differential Scanning Calorimetry (DSC).................. 180

X-Ray Crystal Structure of rVCP......................... 182

VCP is a Monomer in Solution.......................... 187

Shelf-life and Freeze-thawing Tolerance................... 187

Temperature and $\mathrm{pH}$ Tolerance......................... 196

Nuclear Magnetic Resonance (NMR) and Differential

Scanning Calorimetry (DSC) Studies..................... 197

Discussion................................................... 203

\section{PHARMACOKINETICS OF VCP}

Introduction.................................................. 216

Materials and Methods.......................................... 220

Single Injection Strategies.......................... 220

Multiple and Continuous Injection Strategies............. 220

CH50 Hemolytic Activity Assay........................ 221

Detection of rVCP in Urine.............................. 221

Oligomerization and Modification of $\operatorname{rVCP} \ldots \ldots \ldots \ldots \ldots \ldots . \ldots 222$

Results.................................................. 223

Single Bolus Injection Studies....................... 223

Multiple and Continuous Injection Studies................ 231

Presence of rVCP in Urine................................. 232

Attempts to Oligomerize and Modify rVCP.............. 235

Discussion............................................... 238 
Species Specificity Studies of rVCP and the Variola Virus

Homologue

rVCP Cell Binding and Kinetic Studies Using Electron

Microscopy

Pharmacokinetic and Xenotransplantation Studies in Baboons....... 246

$\mathrm{C} 3 \mathrm{~b} / \mathrm{rVCP}$ and Heparin/rVCP Co-crystallization Studies............ 247

$\mathrm{C} 3 \mathrm{~b} / \mathrm{rVCP}$ and Heparin/rVCP Binding Kinetics Using Biacore....... 248

Identification of C3 Fragments Involved in rVCP Binding Using

Biacore.

Protein Engineering and Use of VCP as an Immunomodulatory

Agent. 250

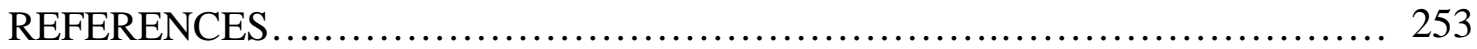

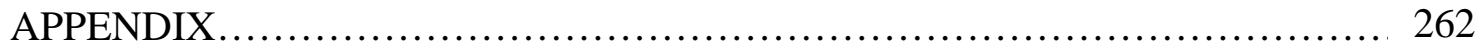

CURRICULUM VITAE.................................................. 275 


\section{LIST OF FIGURES}

FIGURE

PAGE

1. Vaccinia virus genome organization................................ 4

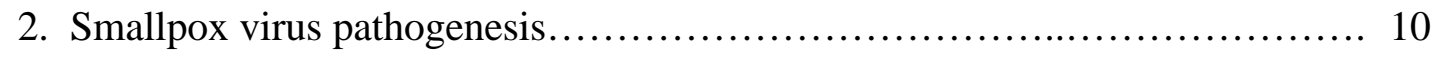

3. Life cycle of the vaccinia virus................................... 14

4. Important sequences for transcription and concatemer resolution............. 19

5. Poxvirus immune modulation....................................... 25

6. Pichia pastoris yeast expression system.............................. 41

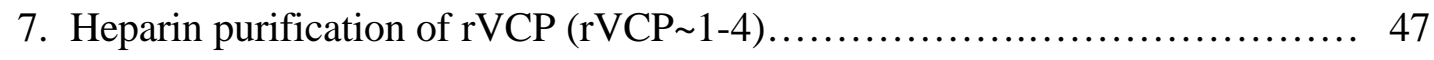

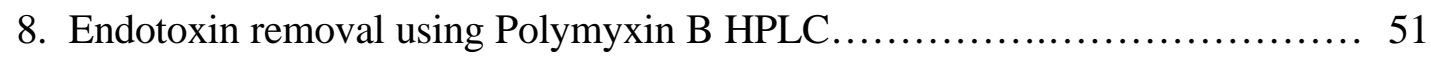

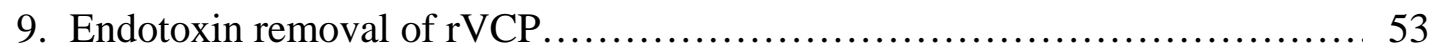

10. Hemolysis assay.............................................. 56

11. Heparin affinity chromatography of ammonium sulfate $\mathrm{rVCP} \ldots \ldots \ldots \ldots \ldots \ldots$

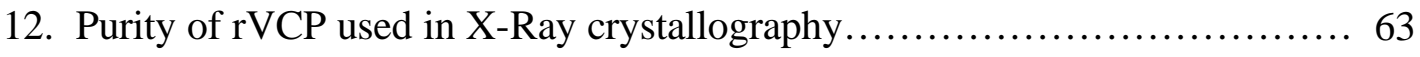

13. Purification and characterization of anti-rVCP IgY..................... 66

14. Heparan sulfate proteoglycans................................... 70

15. Standard amine coupling procedure for Biacore instrument............... 77

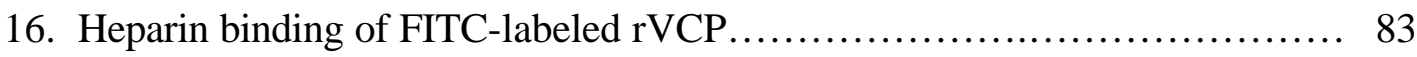

17. Representative histograms showing that VCP is able to reduce mouse antihuman HLA class I antibody binding to HUVECs................... 85 
18. PAGE analysis of the heparin binding activity of BSA, HBP, lysozyme,

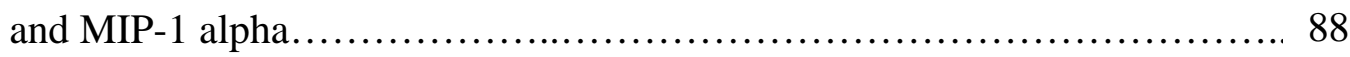

19. PAGE analysis of the heparin binding activity of VCP and rVCPs.......... 90

20. Sequence alignment including termini of rVCP constructs and putative heparin binding sites......................................... 93

21. Structure-function summary table of VCP, VCP homologues, and rVCPs.... 95

22. Heparin binding of rVCP fragments.............................. 98

23. Heparin binding of rVCP in presence of KRR tri-peptide................. 101

24. Heparin binding of rVCP in presence of DID tri-peptide.................. 103

25. Heparin binding of rVCP in presence of GSS tri-peptide.................. 105

26. Heparin purification of $\mathrm{rVCP} \sim 1,2, \mathrm{rVCP} \sim 2,3$ (using KRR),

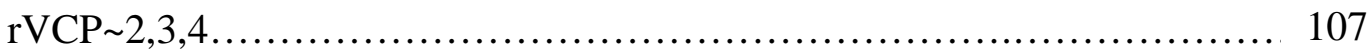

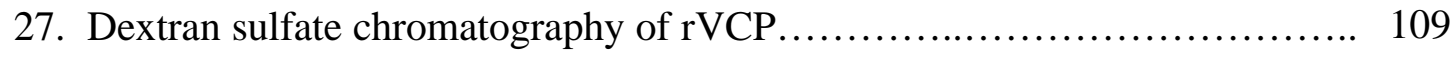

28. VCP model showing the heparin binding sites........................ 112

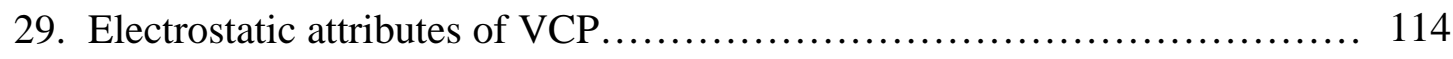

30. Stereo view of interaction of VCP with heparin $\ldots \ldots \ldots \ldots \ldots \ldots \ldots \ldots \ldots \ldots \ldots \ldots$

31. Residues involved in binding heparin in VCP....................... 123

32. Several members of the complement control protein (CCP) family........... 128

33. Complement cascade and points of VCP inhibition..................... 130

34. C3 purification using BioCAD 20HQ column.......................... 137

35. Hemolysis assay of various animal sera............................. 142

36. Hemolysis assay of rat sera using VCP and rVCP...................... 144

37. Structure-function summary table of VCP, VCP homologues, and rVCPs.... 146 
38. Control $1^{\circ}$ and $2^{\circ}$ antibody binding ................................... 149

39. Coomassie blue stained SDS-PAGE of C3, C3b, and rVCP constructs........ 151

40. C3b binding of rVCP fragments...................................... 153

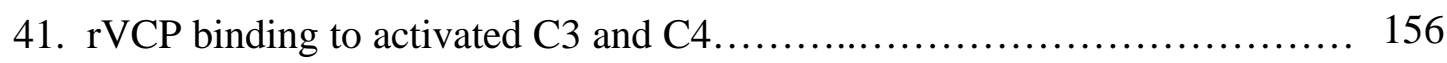

42. Simultaneous heparin binding and hemolysis inhibition by $\mathrm{rVCP} \ldots \ldots \ldots \ldots \ldots 158$

43. Simultaneous heparan sulfate binding and hemolysis inhibition by rVCP...... 160

44. Simultaneous binding to heparin and complement by $\operatorname{rVCP} \ldots \ldots \ldots \ldots \ldots \ldots \ldots$

45. Model for simultaneous heparin and complement binding.................... 165

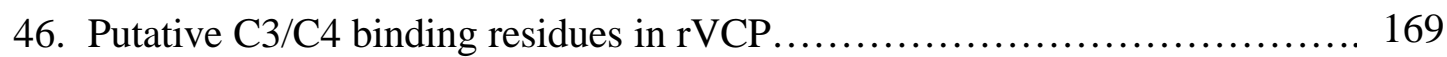

47. Packing in form I and form II crystals.................................... 184

48. Five independent molecules are similar................................ 186

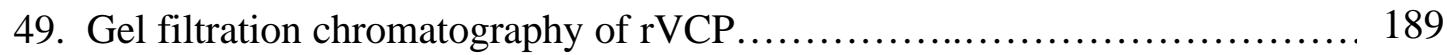

50. Gel filtration chromatography of rVCP and marker proteins................ 191

51. rVCP tolerance to extreme temperature and shelf-life....................... 193

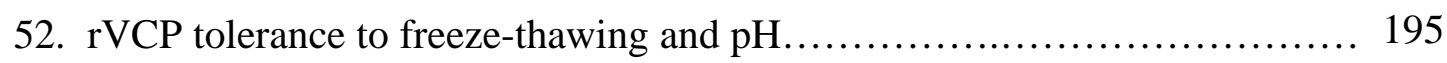

53. Differential scanning calorimetry profiles..................................... 199

54. NMR spectroscopy .............................................. 202

55. Diverse orientations of modules in CCP containing proteins................. 205

56. (A) Extensive H-bonding within VCP structure contributes to its stability.... 209

(B) Extensive H-bonding within VCP structure contributes to its stability.... 210

57. Disulphide bonding pattern within CCP modules of VCP.................. 213

58. CH50 following I.V. and I.P. injection of $3 \mathrm{mg}$ rVCP.................... 225

59. Serum CH50 of I.V. injected rVCP at 3, 6 and $12 \mathrm{mg} / \mathrm{kg} \ldots \ldots \ldots \ldots \ldots \ldots \ldots . \ldots 227$ 
60. Baboon 479 and 397 CH50 following $9 \mathrm{mg} / \mathrm{kg} \mathrm{I.V.} \mathrm{rVCP.................} 230$

61. Presence of rVCP in urine........................................... 234

62. Modification of rVCP using standard amine coupling ..................... 237

63. Rat serum CH50 following $24 \mathrm{mg} / \mathrm{kg}$ SubQ bolus........................ 264

64. Baboon $479 \mathrm{CH} 50$ following $9 \mathrm{mg} / \mathrm{kg} \mathrm{I.V.} \mathrm{bolus.......................} 266$

65. Rat serum CH50 following continuous $16 \mathrm{mg} / \mathrm{kg} / \mathrm{hr}$ via catheter............ 268

66. Rat serum CH50 following $6 \mathrm{mg} / \mathrm{kg} \mathrm{I.V.}+3 \mathrm{mg} / \mathrm{kg} / \mathrm{hr}$ via pump............ 270

67. Rat Serum CH50 following $6 \mathrm{mg} / \mathrm{kg} \mathrm{I.V.}+6 \mathrm{mg} / \mathrm{kg} / \mathrm{hr}$ via pump............ 272

68. Rat Serum CH50 following $6 \mathrm{mg} / \mathrm{kg} \mathrm{I.V.}+6 \mathrm{mg} / \mathrm{kg} / \mathrm{hr}$ via catheter.......... 274 


\section{LIST OF ABBREVIATIONS}

antibody-dependent cell-mediated cytotoxicity .................................. ADCC

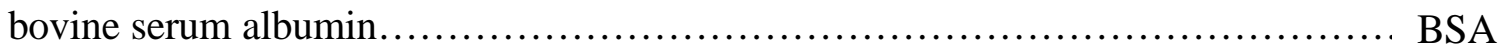

buffered minimal-complex glycerol medium................................... BMGY

buffered minimal-complex methanol medium.................................. BMMY

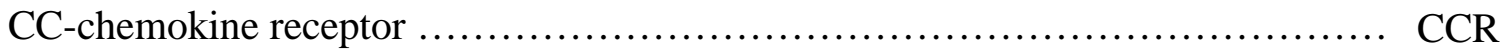

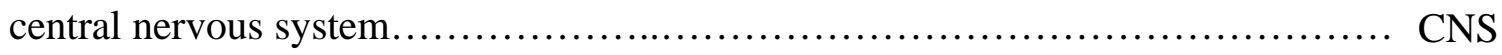

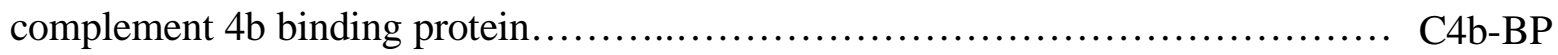

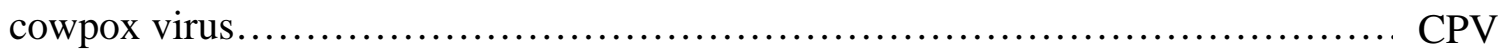

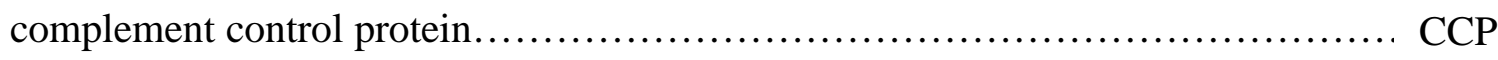

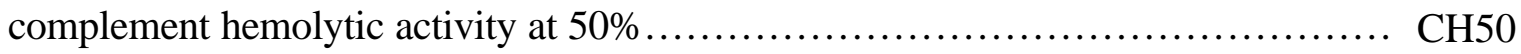

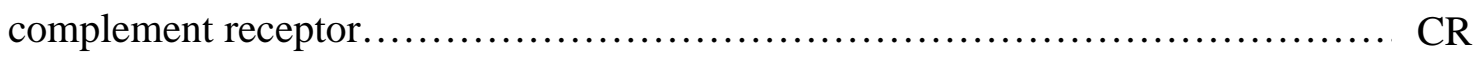

cowpox cytokine response modifier A ..................................... CrmA

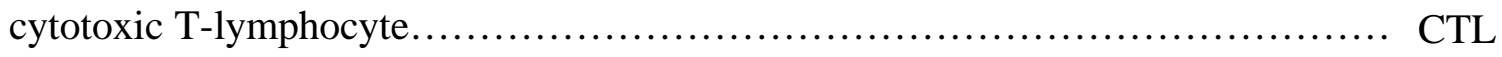

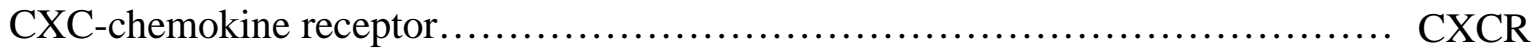

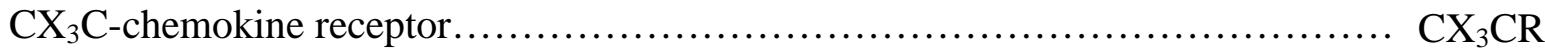

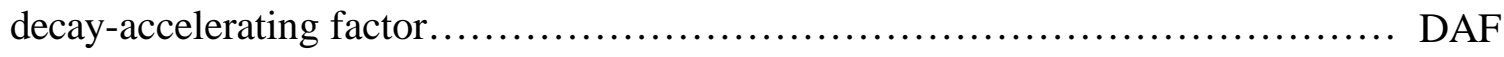

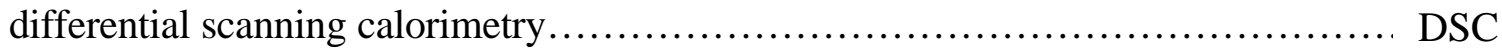

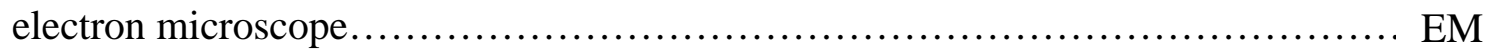

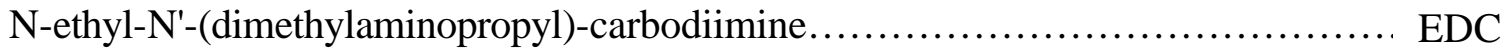

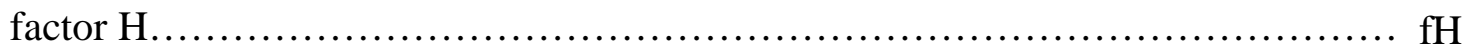




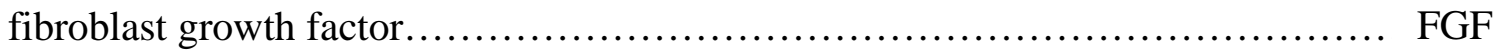

fluorescein-isothiocyanate...................................................... FITC

granulocyte-macrophage colony-stimulating factor-inhibitory factor.............. GIF

granulocyte-macrophage colony-stimulating factor............................ GM-CSF

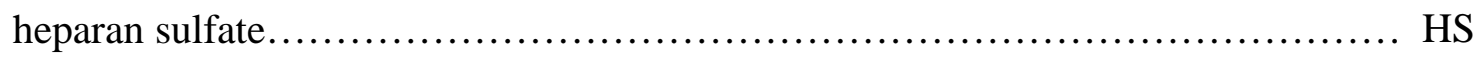

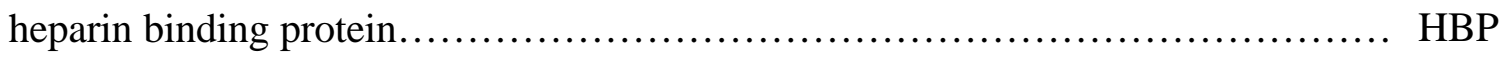

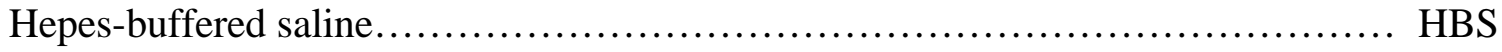

human leukocyte antigen................................................... HLA

human umbilical cord vascular endothelial cells................................... HUVECs

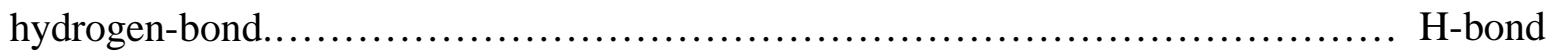

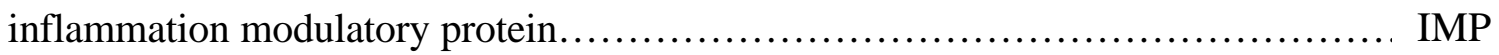

interleukin- $1 \beta$-converting enzyme.......................................... ICE

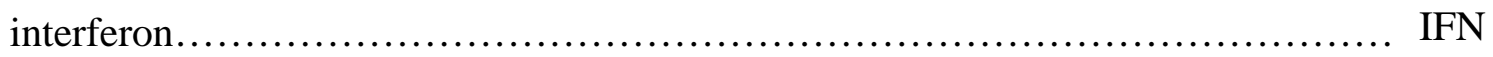

interferon receptor............................................................ IFNR

interleukin......................................................................... IL

interleukin 1 receptor......................................................... IL

interleukin 18 binding protein............................................. IL-18BP

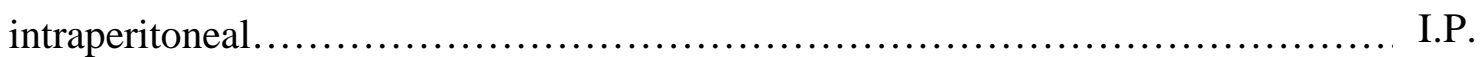

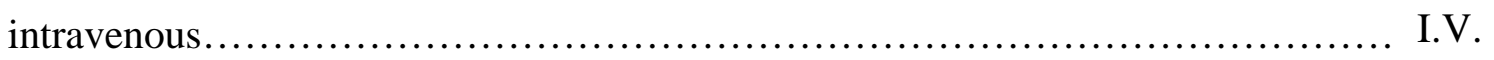

mean fluorescent intensity.................................................... MFI

membrane attack complex.................................................. MAC

membrane cofactor protein..................................................

molluscum contagiosum chemokine homologue................................ MCCH 
molluscum contagiosum virus...

monkeypox virus

MPV

myxoma virus growth factor.

MGF

major histocompatibility complex.

$\mathrm{MHC}$

macrophage inflammatory protein.

MIP

myxoma virus.

MV

natural killer.

NK

N-hydroxysuccinimide.

NHS

nuclear factor

NF

nuclear magnetic resonance.

NMR

open reading frame.

ORF

phenylmethylsulfonyl fluoride....

PMSF

polyethylene glycol.

PEG

pig aortic endothelial cells

PAECs

recombinant vaccininia virus complement control protein

rVCP

regulators of complement activation

RCA

root mean square.

$\mathrm{rms}$

sensitized sheep red blood cell. SSRBC

serine protease inhibitor.

Serpin

Shope fibroma virus.

SFV

short consensus repeats.

SCR

smallpox inhibitor of complement enzymes.

SPICE

sodium dodecyl sulfate-polyacrylamide gel electrophoresis

SDS-PAGE 
soluble complement receptor one....

SCR1

soluble tumor necrosis factor receptor.

sTNFR

subcutaneous

SubQ

surface plasmon resonance

SPR

transforming growth factor

TGF

tumor necrosis factor

TNF

tumor necrosis factor receptor.

TNFR

tissue-type plasminogen activator.

tPA

urokinase-type plasminogen activator

UPA

vaccinia virus

VV

vaccinia virus complement control protein.

VCP

vaccinia virus growth factor

VGF

viral interferon receptor.

vIFNR

viral interleukin.

vIL

viral interleukin 1 receptor

vIL-1R

viral interleukin 18 binding protein

vIL-18BP

viral macrophage inflammatory protein

vMIP

viral tumor necrosis factor receptor.

vTNFR

World Health Organization.

WHO

C-chemokine receptor.

$\mathrm{XCR}$ 


\section{CHAPTER 1}

\section{POXVIRUSES: VACCINIA VIRUS}

\section{$\underline{\text { Introduction }}$}

This chapter contains material adapted from the following publications:

Smith S.A., and Kotwal G.J. Virokines: novel immunomodulatory agents. Exp. Opin. Biol. Ther., 1(3):343-357, 2001.

Smith S.A., and Kotwal G.J. Vaccinia virus. In: Encyclopedia of Molecular Medicine. John Wiley \& Sons Inc., Vol. 5, pp. 3324-3330, 2001.

Smith S.A., and Kotwal G.J. Immune response to poxvirus infections in various animals. Crit. Rev. Microbiol., In press, 2002.

Vaccinia virus (VV), the most widely studied member of the poxvirus family, serves as a model to enhance our understanding of this extraordinary family of complex DNA viruses. Like other poxviruses, which have the largest virions of any animal virus family, VV virions are substantial in size. They appear as smooth, rectangular sacks, approximately $350 \mathrm{~nm}$ in length by $270 \mathrm{~nm}$ in width. Because of its relatively large size, VV was the first animal virus to have beeen seen microscopically, in 1886. Vaccinia virus contains a large, linear, double-stranded DNA genome of over $191 \mathrm{kbp}$. This large genome is responsible for encoding all the proteins necessary for vaccinia's unique cytoplasmic lifestyle. Like other members of the poxvirus family, VV replicates almost entirely independent of the infected cell nucleus. Therefore, being unable to utilize the host cell's machinery to synthesize RNA or replicate DNA, VV must encode these 
important proteins in its own genome. Another interesting feature of the vaccinia genome is the presence of terminal hairpin loops. The ends of the double-stranded DNA are covalently linked to form a continuous single-stranded polynucleotide chain (figure 1). This unique telomeric structure seems to be very important in replication.

Upon entry into the host cell, the VV life cycle is naturally divided into three stages according to gene expression: early, intermediate, and late. Early genes are expressed via transcriptional regulatory proteins packaged within the infecting virion. It is during this time that the genome is replicated to high numbers in cytoplasmic factories (virosomes) through the formation of large concatemers, utilizing many proteins produced during this early stage of gene transcription. The intermediate stage of vaccinia replication is defined by a strong spike in gene expression, falling shortly after the initiation of DNA replication and before the expression of the more abundant late genes. Late gene expression is responsible for production of the major virion components, including those that are structurally important and those necessary for early stage gene expression upon entry into a new host cell. Late gene expression continues to produce structural proteins that accumulate in large amounts in the cytoplasm, allowing for viral assembly and maturation for at least 48 hours after infection.

In addition to those viral proteins encoded by VV required for transcription, replication and virion assembly, there are several nonessential proteins utilized by VV to establish a suitable habitat. Vaccinia virus encodes a growth factor that, when secreted from infected cells, results in the proliferation of neighboring cells, increasing the number of local cells that can be infected by viral progeny. Other interesting nonessential proteins encoded by vaccinia virus include: complement control protein (VCP), 
Figure 1. Vaccinia virus genome organization. The vaccinia virus genome is a very large double stranded DNA. The end $5 \%$ of the genome is made up of inverted terminal repeats and the very ends are covalently closed by terminal hairpin loops. The genome is divided into three main areas: variable left end, conserved central region, and variable right end. Listed are many of the important proteins coded by these areas of the vaccinia virus genome. 


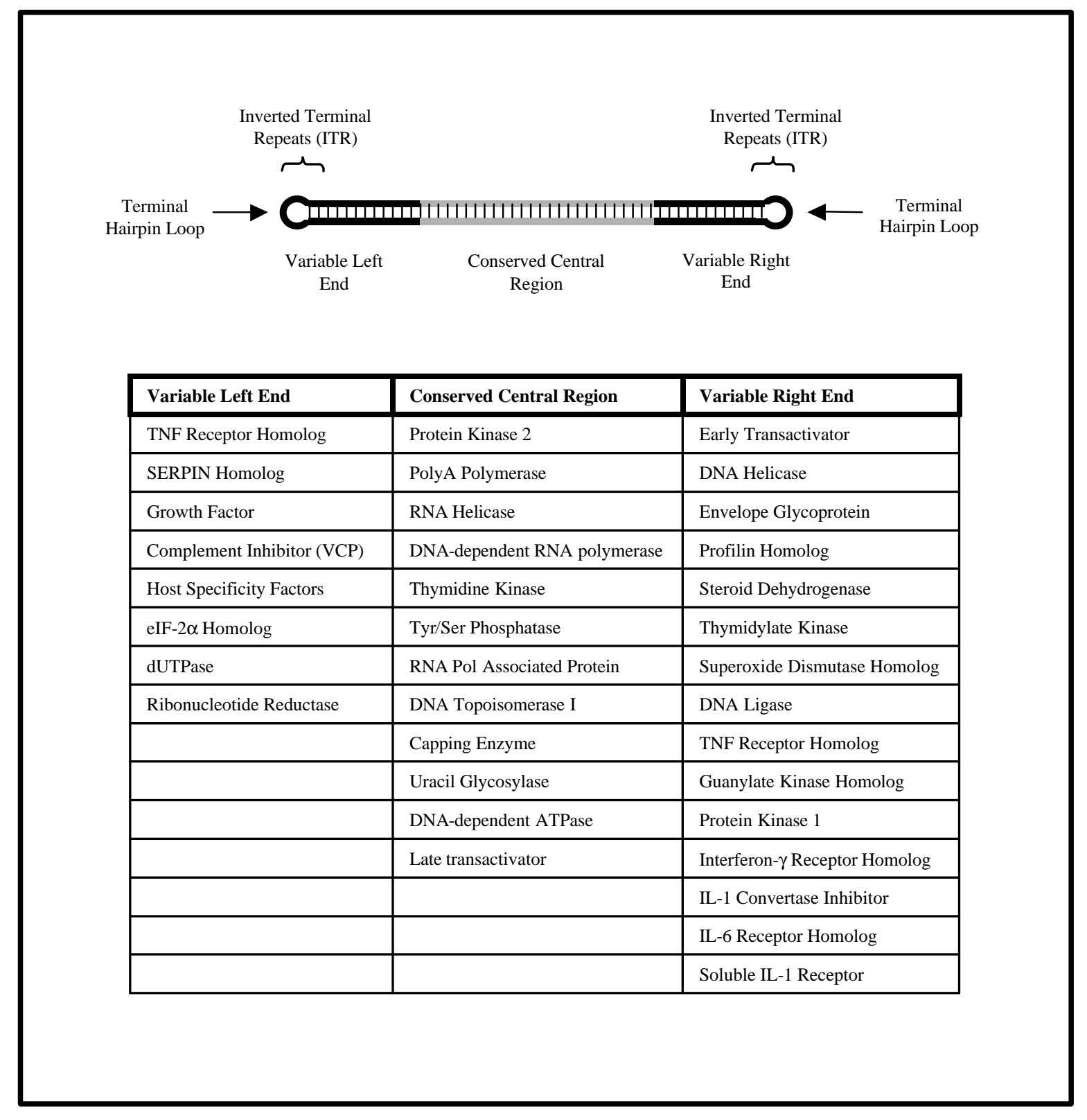


interleukin one receptor (IL-1R) homologue, tumor necrosis factor receptor (TNFR) homologue, and interferon receptor (IFNR) homologue. Although not all of the nonessential proteins have been studied, each is presumed to play an important role in modulating the host's immune response to infection, thereby maintaining the viral habitat.

Vaccinia virus also played an important role in the eradication of the deadly variola virus. Edward Jenner, in 1796, started immunizing individuals with a very close relative of $\mathrm{VV}$, cowpox virus (CPV). Eventually, $\mathrm{VV}$ replaced $\mathrm{CPV}$ as the chosen vaccine, and the World Health Organization (WHO) launched its extremely successful eradication campaign. Interestingly, the exact origin of VV still remains a mystery today. It is postulated that $\mathrm{VV}$ arose in the $19^{\text {th }}$ or early $20^{\text {th }}$ centuries from all the mixing of vaccine strains (or possibly variola and cowpox), since the nature of viruses was still quit unknown at that time. Therefore, it is still believed that the lack of a natural host reservoir and broad host range exhibited by vaccinia virus is due to its creation in a laboratory.

As mentioned earlier, the poxvirus family, Poxviridae, has the largest virions of any animal virus family. They appear oval or brick shaped, approximately $350 \mathrm{~nm}$ in length and $270 \mathrm{~nm}$ in width. Poxviruses contain a large, linear, double-stranded DNA genome of $130-280 \mathrm{kbp}$. This large genome is responsible for encoding all the proteins necessary for their unique cytoplasmic lifestyle. Members of the poxvirus family replicate almost entirely independent of the nucleus of the infected cell. Unable to utilize the host cell's enzymes to synthesize RNA or replicate DNA, they encode these essential proteins in their own genome. In addition to those proteins encoded by poxviruses required for transcription, replication and virion assembly, there are several nonessential 
proteins employed to establish a suitable habitat. Several interesting nonessential proteins encoded by poxviruses include: complement control protein, IL-1R homologue, TNFR homologue, and IFNR homologue (Kotwal, 1997). These nonessential proteins, and several others, play an important role in modulating the host's immune response to the infection, thereby maintaining the viral habitat (Howard et al., 1998).

The family Poxviridae is divided into two subfamilies, Chordopoxvirinae, which are vertebrate poxviruses, and Entomopoxvirinae, which are insect poxviruses - we will only discuss the Chordopoxvirinae (Moss, 1996). There are eight genera of vertebrate poxviruses - see table 1. Poxviruses have been found to infect vertebrates ranging from birds to humans. Some of these infections can be highly fatal; smallpox virus (variola), which replicates only in humans, has killed more people than any other infectious disease. In addition, myxoma virus, which infects rabbits, can have as high as a 99\% mortality rate. Much of the success of poxviruses as pathogens is due to the immune evasion strategies they employ (Kotwal, 1997).

The poxvirus infection generally follows one of two courses, a localized infection resulting in benign skin lesions, or a systemic infection resulting in viral dissemination and usually death. Local pathogenesis of molluscum contagiosum virus (MCV) and Shope fibroma virus (SFV) infection has been well documented. Although the pathogenesis and host response to infection is quite different, both poxvirus diseases are self-limiting and remain localized to the skin. The generalized infections, however, are characterized by several stages, ending in an explosion of viral lesions. Ectromelia virus infection of mice starts with an infection of the skin, usually via the footpad. After some replication, the virus spreads through the local lymphatics to the bloodstream, resulting in 
Table 1. Poxvirus classification. The family Poxviridae, subfamily Chordopoxvirinae is composed of eight genera. Listed are the most studied members of each genus.

\begin{tabular}{|c|c|c|c|c|}
\hline \multicolumn{5}{|c|}{ Family Poxviridae, Subfamily Chordopoxvirinae } \\
(Vertebrate poxviruses)
\end{tabular}


primary viremia. The virus then replicates to high titers in the spleen and liver before reentering the blood, resulting in a secondary viremia. Finally, the virus spreads to the skin and a severe rash of ulcerating lesions very rapidly overcomes the mouse. The disease progression of variola virus infection in humans occurs similarly, with high mortality rates - see figure 2 .

The immune response to poxvirus infection is very complex. Each arm of the immune system plays a vital role in the segregation and elimination of a poxvirus infection. Early in the infection, the innate immune response attempts to keep the infection under control, while the acquired immune response is mounted. Complement, interferon (IFN), NK and inflammatory cells help control the early infection. Within a few days, a very strong humoral immune response is mounted, and poxvirus-specific antibodies are produced. The antibodies can then help control the infection through a variety of mechanisms, including virus neutralization and complement activation, opsonization, and ADCC. In addition, a cell-mediated immune response is mounted a few days into the infection. Poxvirus-specific CTLs are generated and attack virusinfected cells, ultimately resulting in clearance of the poxvirus infection. Animal models have been used quite extensively for the study of poxvirus disease pathogenesis and exploring the therapeutic potential of poxvirus immunomodulatory proteins. To gain a better understanding of the infection processes and genes involved in interactions with the host, several animal models have been developed to study various poxvirus diseases and recombinant mutant poxviruses. Animal infection models are important for testing the efficacies of new anti-viral drugs or to explore new treatment strategies. Models have been developed to study the inflammatory response generated by 
Figure 2. Smallpox virus pathogenesis. Smallpox virus infection occurs via the respiratory route or direct skin-to-skin contact with a lesion. An incubation period of approximately 10 to 12 days follows while the virus replicates in the draining lymph node(s). After this incubation period, the virus enters the blood and disseminates throughout the body, causing fever, malaise, headache and backache; these are the first signs of sickness. For the next 3 to 6 days, the virus can be recovered from the patient's blood. An eruptive exantham then ensues, starting as small macular lesions of the mouth and pharynx, face and forearms, before spreading to the trunk and legs. The rash becomes popular, then pustular and often protrudes from the skin before scabbing over on the eighth or ninth day of the rash. If the individual recovers, permanent damage to the skin (pockmarks) becomes evident. Overall there is a $20-50 \%$ mortality rate associated with smallpox infection, however, in the rapidly progressing type of infection, mortality may be as high as $95 \%$. 


\title{
Smallpox Pathogenesis
}

\author{
Exposure \& replication \\ in local lymph nodes
}

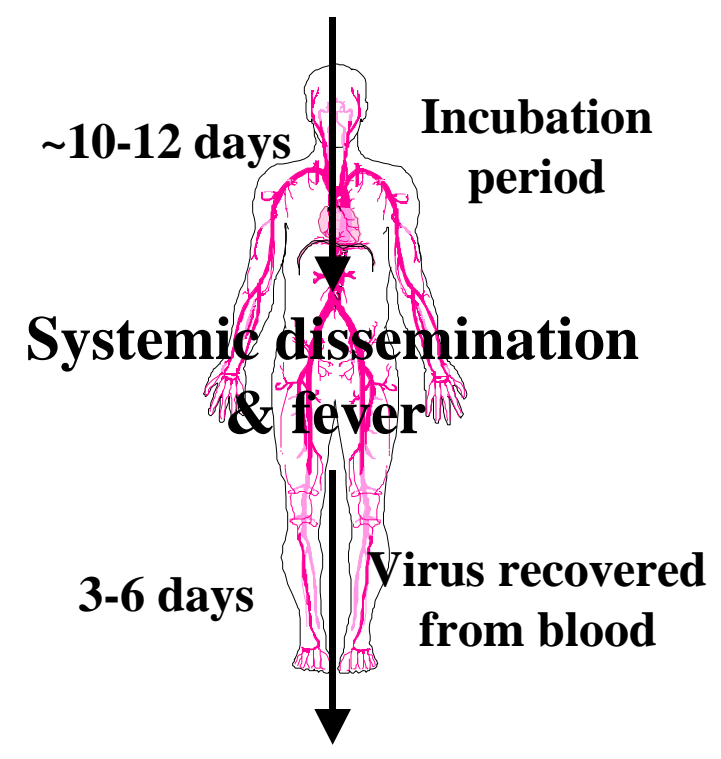

Eruptive exanthem

(Photographs from the World Health Organization)

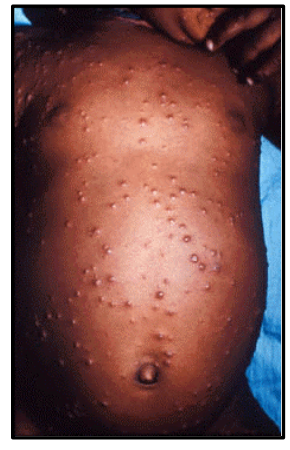

Day 3 of rash

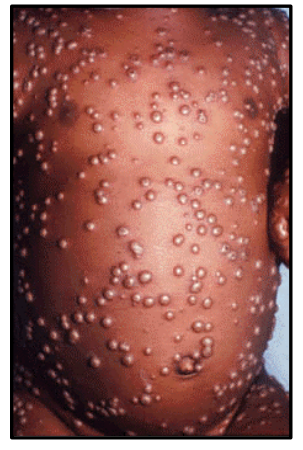

Day 5 of rash

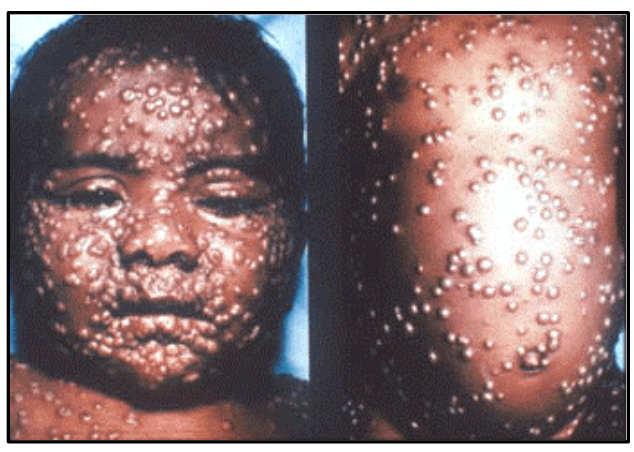

Day 7 of rash

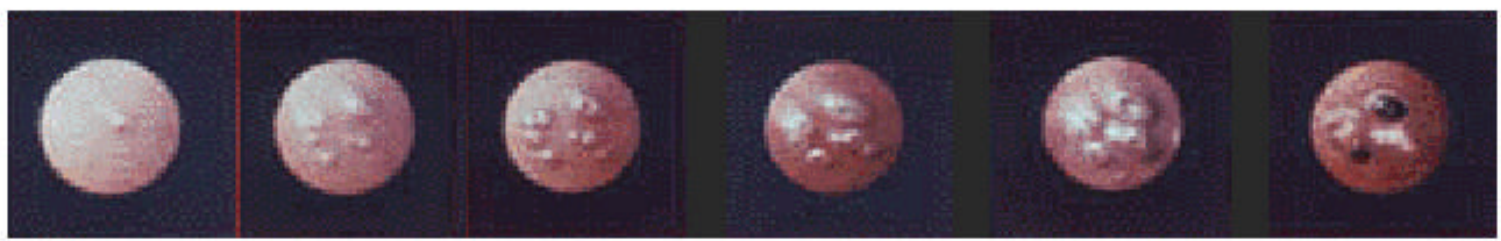

Macules

$\longrightarrow$ Papules

Pustular vesicles Scabs 
poxvirus infection, analyzing the degree of inflammation and cellular infiltration both quantitatively and qualitatively. This allows for a better understanding of the genes associated with poxvirus pathogenesis and results in better characterization of mutant strains. Understanding pathogenesis assists in the generation of more attenuated vaccine strains and poxvirus vectors for use in live vaccines, in hopes of reducing complications associated with traditional vaccines. Animal models have also been instrumental in the study of poxvirus immune modulating proteins. Several poxvirus immunomodulatory proteins have been investigated extensively for their potential use as therapeutic agents. These immune blocking proteins have very powerful anti-inflammatory properties that may be employed for treatment of many injuries or diseases that involve excessive inflammation. Animal model systems have been used to test the value of these proteins for use in transplantation, vascular injury, CNS injury, sepsis, arthritis, and restenosis. The therapeutic effects of these proteins, revealed by these model systems, are often quite fascinating. 


\section{$\underline{\text { Virus Structure }}$}

Several methods utilizing electron microscopy have been used to visualize viral particles. Thin section electron microscopy (often using immunostaining) and cryoelectron microscopy are widely used and have played a very important role in deciphering both VV morphology, as well as the various intracellular stages of its life cycle. Vaccinia virus virions appear as large, smooth surfaced rectangles ( $350 \mathrm{~nm}$ by 270 nm) when their surface is viewed by cryoelectron microscopy. When fixed, stained, and viewed as thin sections, the vaccinia virions appear much more elliptical. The dumbbellshaped virion core (within which resides the large double-stranded DNA genome and essential proteins) can also be seen through this method along with the lateral bodies (unknown function) lying adjacent, within its cavities. This characteristic shape is now believed to be an artifact caused by dehydration during section preparation. Regardless of the method used, however, a $30 \mathrm{~nm}$ external surface membrane can be clearly seen surrounding the entire virion.

One very interesting feature of the VV is how it makes many intracellular and extracellular morphological forms, two of which are infectious. During virion maturation, several structurally unique forms can be discerned by electron microscopy: the immature virus (IV), the infectious intracellular mature virus (IMV), the intracellular enveloped virus (IEV), the cell-associated enveloped virus (CEV), and the infectious extracellular enveloped virus (EEV) - see figure 3. The immature virus (IV) occurs early and is characterized by a single outer membrane and incompletely formed core. The intracellular mature virus (IMV) also contains a single outer membrane; however, its core is completely formed, allowing the virus to be infectious when released by cell lysis 
Figure 3. Life cycle of the vaccinia virus. (1) Either of the two infectious forms of vaccinia virus (IMV or EEV) may bind a receptor on a host cell, allowing for membrane fusion, and viral core entry into the cytoplasm of the cell. (2) Essential viral core proteins are then able to initiate early gene transcription from the viral genome (completely independent of the host cell's nucleus). (3) Early viral mRNA is translated by host cell ribosomes into early proteins (many are involved in inhibition of the host's immune response). (4) Many early proteins aid in the replication of the vaccinia virus genome. This replication involves the formation of large concatomeric structures. (5) After the genome is replicated to high numbers, transcription factors (early protein) and a cellular protein (Vitf2), relocating from the nucleus, allows for transcription of intermediate genes from the newly synthesized genomes. (6) These intermediate mRNAs are then translated to proteins by host cell ribosomes. (7) Transcription factors produced during the intermediate stage then lead to late gene transcription. (8) Again, the host cell ribosomes translate the late vaccinia virus mRNAs into late proteins. (9) Late structural proteins and mature genomes assemble on intracellular membranes and form immature virus particles (IV). (10) Cleavage of core proteins occurs and the IV becomes an intracellular mature virus (IMV). (11) The IMV may escape the host cell by lysis and infect a neighboring cell. (12) Alternatively, the IMV may bud through the golgi network and acquire two additional membranes and becomes an intracellular enveloped virus (IEV). (13) The IEV can then polymerize actin and push to the cell membrane, where it fuses and becomes known as a cell-associated enveloped virus (CEV). The CEV may bud free of the host cell, losing one of its three membranes and becoming known as an extracellular enveloped virus (EEV). This form of vaccinia virus is also infectious and may be important in dissemination. 


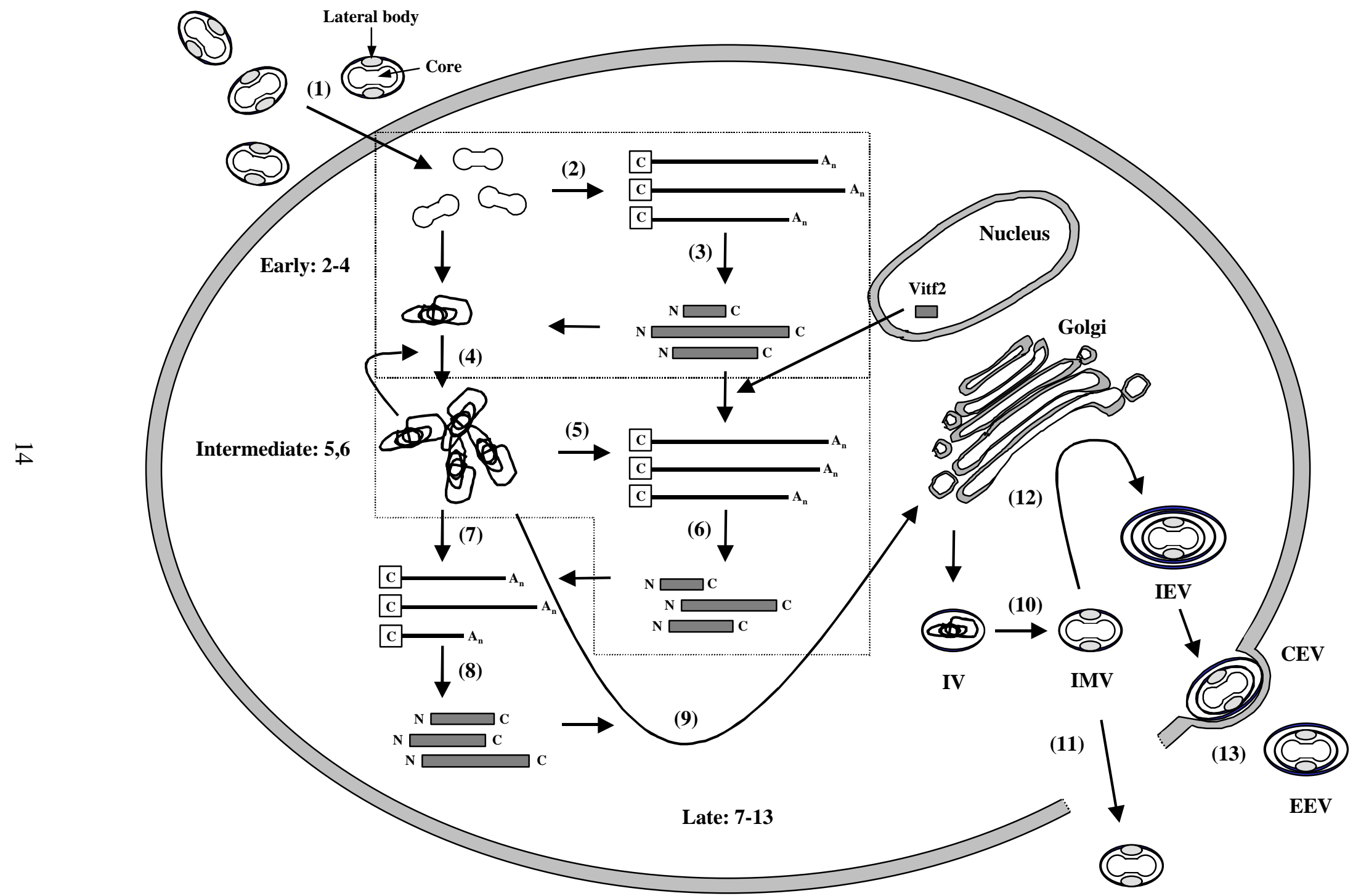


(Hollinshead et al., 1999). After acquiring two additional membranes, the IMV becomes known as the intracellular enveloped virus (IEV), possessing three membranes in total. The IEV can move to the plasma membrane (through actin polymerization), fusing to become known as the cell-associated enveloped virus (CEV). This morphological form may be released by further budding through the membrane, losing one of its membranes, and is known as the extracellular enveloped virus (EEV). This form of VV is also infectious and is responsible for cell-to-cell transmission as well as dissemination throughout the host. 


\section{Genome Organization}

The complete sequence of VV, Copenhagen strain, has been known for many years now. This large linear, double-stranded genome was found to be 191,636 bp, possessing 263 open reading frames, 65 of which are contained within a larger open reading frame (ORF) (Johnson et al., 1993). The genome as a whole is roughly divided into three general regions: variable left end, variable right end, and conserved central region - see figure 1. The variable left and right ends of the genome, for the most part, contain nonessential ORFs responsible for encoding proteins used in host specificity and modulating host defense. Some of these are close homologues of host immune proteins, clearly indicating that genes were derived and adapted from the host cell. The conserved central region of the genome contains ORFs that encode all the essential proteins required for replication in cell culture. This includes those proteins necessary for RNA synthesis, DNA replication, and virion assembly. The extreme ends of the VV genome are composed of identical, but oppositely oriented, inverted terminal repeats. Contained within the $12 \mathrm{kbp}$ inverted terminal repeats found at either end of the genome are several tandem repeating sequences of approximately $70 \mathrm{bp}$ in length.

As mentioned earlier, the far ends of vaccinia virus's DNA are covalently linked hairpin loops, which form a continuous polynucleotide chain. These terminal hairpin loops are composed of highly A+T rich sequence that do not fully base-pair. This telomeric structure allows DNA replication to occur through a continuous simple elongation step, creating large concatemers, resulting in large numbers of replicated genomes. Just adjacent to the terminal loop lies an extremely conserved $100 \mathrm{bp}$ region. This region contains the resolution sequence - see figure 4 - responsible for the precise 
cleavage of these large concatemeric DNA structures into individual genomes, which are complete and ready to be packaged to form an infectious virion. 
Figure 4. Important sequences for transcription and concatemer resolution. Listed are the important nucleotides involved in early, intermediate, and late promoter function. An important spacer region between the core promoter and initiation site, responsible for proper positioning of the polymerase, in noted. The early termination signal, which is highly conserved among early genes, results in termination of the mRNA 20-50 base pairs downstream of this sequence. The efficiency of termination is about $80 \%$. The concatemer resolution site is highly conserved and located at the far end of the genome. Cleavage of large concatemers into genome length fragments occurs specifically at this site. 
Important Sequences for Transcription and Concatemer Resolution.

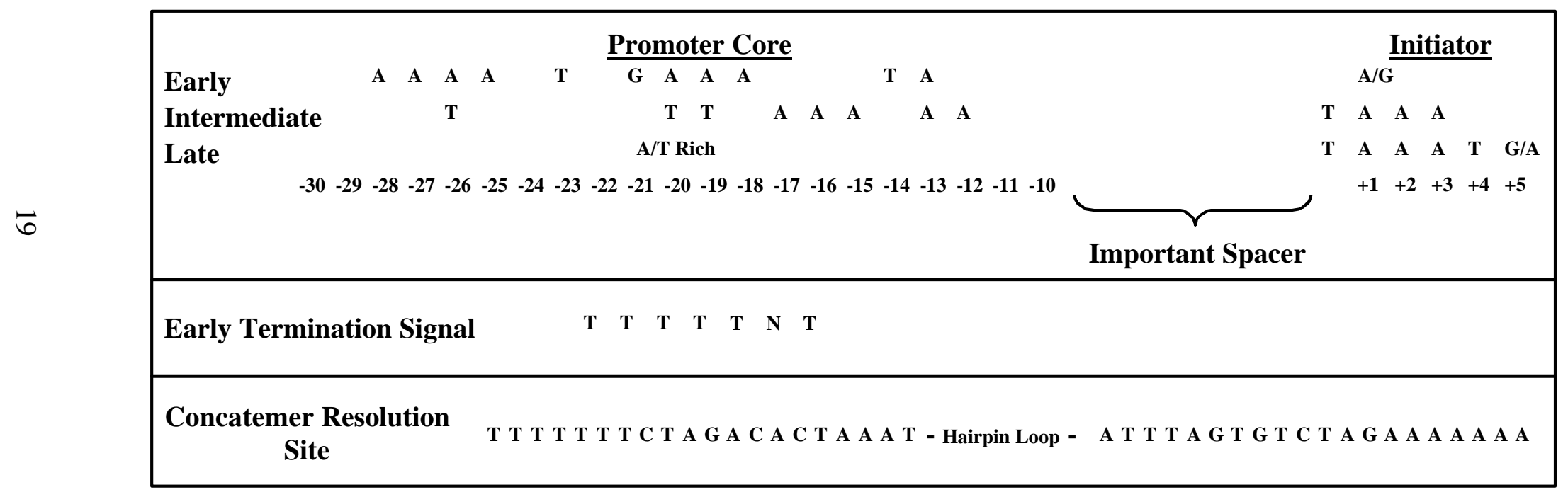




\section{$\underline{\text { Virus Life Cycle }}$}

Vaccinia virus replicates exclusively within the cytoplasm of host cells in three stages temporally regulated by viral transcriptional activating proteins (Beaud, 1995). The various stages of replication - early, intermediate, and late - are summarized in figure 3. Infection is initiated through attachment of the virion, followed by entry of the core into the host cell. The cellular receptor responsible for VV attachment and entry has recently been shown to be heparan sulfate proteoglycan (Chung et al., 1998). A protein present on infectious VV binds this very common cell surface component, allowing for membrane fusion and ultimately core entry. The ubiquitous nature of this receptor helps explain the broad host range exhibited by this virus. As soon as the VV core enters the cytoplasm of the host cell, early transcription is initiated.

Early viral mRNAs, synthesized by the transcription system packaged in the core, are detectable in the cytoplasm within 20 minutes and reach a maximum level between one and two hours after entry. Approximately one hour after entry, intermediate viral mRNAs are detected and reach a maximum level in two hours. In addition, two hours after infection, late viral mRNAs can be detected and will continue to be expressed until the end of infection, approximately 48 hours after initial entry. Various transcriptional activating proteins control the temporal regulation of these various stages of gene expression. Early gene expression occurs through the specific promoter binding (figure 4) of early transcription activating proteins, produced and packaged into infectious virions during the late stage of infection. Intermediate gene expression occurs similarly through the specific promoter binding of intermediate transcription activating proteins, 
produced during the early stage of gene expression. Late genes are expressed through the binding of transactivating proteins produced in the intermediate stage of infection.

During early transcription, all of the necessary proteins required for DNA replication are synthesized and immediately utilized to replicate the genome. Large concatemers of DNA are thereby synthesized within cytoplasmic foci often referred to as factory areas or virosomes. The process occurs with very little help from the host cell nucleus. This has been demonstrated through various experiments using enucleated cells. Genome replication occurs very rapidly and results in around 10,000 new genomes per cell (only half of which will be packaged into virions). The speed at which new DNA can be synthesized is greatly enhanced by several viral enzymes involved in synthesizing deoxyribonucleotides, increasing the cytoplasmic precursor pools. The large concatemers that result are then cleaved into genome length segments by a specific enzyme that recognizes a region of DNA located near the terminus of the mature genome. The specific enzyme responsible for this is still not known. The most likely candidate, however, seems to be the virally encoded DNA topoisomerase I enzyme, known for sitespecific cleavage of DNA (Berger, 1998).

Once late transcription has been initiated, and structural proteins accumulate in the cytoplasm, mature genomic DNA can be packaged along with proteins required for early gene transcription. The assembly process probably occurs in association with an internal host cell membrane, presumably the Golgi network. After acquiring a double membrane through association with the Golgi network, an immature virus (IV) is formed. Further maturation from this immature state then results in the development of an intracellular mature virus (IMV) that is fully capable of binding and infecting other cells, 
but only through lysis of the host cell. Alternatively, this IMV may acquire two additional double membranes via the golgi network (three double membranes total), becoming an intracellular enveloped virus (IEV) (Hollinshead et al., 1999; Wilton et al., 1995). By polymerizing cellular actin, the IEV is able to force itself to the cell surface, allowing the outermost membrane to fuse with the plasma membrane, where it becomes known as a cell-associated enveloped virus (CEV). The CEV is now capable of escaping the host cell and disseminating, or may pass directly into a neighboring cell. The extracellular enveloped virus (EEV) that results from budding out of the host cell still retains two double membranes and is capable of binding and infecting a new host cell. 


\section{$\underline{\text { Poxvirus Immunomodulation }}$}

In order to defend themselves from the many arms of the immune system, poxviruses must constantly evolve new, more powerful methods of modulating the host immune response. Being large DNA viruses, poxviral genomes have enough capacity to encode proteins not directly involved in replication. Therefore, they often encode a repertoire of proteins involved in habitat formation and immune evasion - see figure 5. These proteins cover a wide range of activities, reflecting the scope and complexity of the immune response that they counter in their host. The survival of the virus depends on its ability to create and perfect strategies to conceal itself from this much larger and more sophisticated opponent. The creativity and potency of the resulting proteins is fascinating and cover both intracellular and extracellular processes. Thus far, most of the proteins that have been discovered are secreted and extracellular. The term "virokine" was coined and used to describe virally encoded proteins that are secreted from the infected host cell. Most virokines are powerful viral immune modulating proteins created by capturing and modifying genes responsible for regulating the host immune response. These viral proteins therefore share many structural and functional features similar to that of the host proteins. However, since there is limited room within the viral genome, only those genes very useful to the virus are possibly maintained and constantly adapted to make them smaller and more potent. There have been virokines discovered and shown to modulate many important aspects of the immune response. Many cytokine (IL-6, IL-10) and

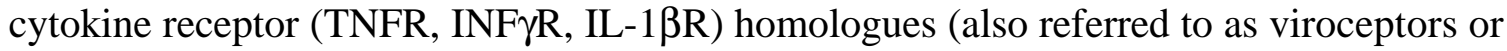
virokines that mimic receptors) have been shown to significantly disrupt cytokinesignaling pathways. This often results in the masking of the host immune system from 
Figure 5. Poxvirus immune modulation: (1) Poxvirus binds the host cell, allowing for core entry and early gene transcription to initiate. At this time, many viral proteins are produced to interfere with the host's immune response, in order to establish a suitable habitat for optimal growth. (2) Vaccinia virus complement control protein (VCP) is produced and secreted from infected cells. VCP can block the activation of complement through its ability to bind $\mathrm{C} 3 \mathrm{~b}$ and C4b complement components. VCP can also bind heparan sulfate proteoglycans on the cell surface, blocking many molecular and cellular interactions with the cell, like chemokine and antibody binding. (3) Poxviruses also produce a soluble interleukin one receptor homolog (vIL$1 \beta R$ ) that is secreted from the cell, binding up soluble IL-1 $\beta$ before it has a chance to reach the cell's receptor. (4) Chemokine binding proteins, such as T1 and T7, inhibit chemokine binding to heparan sulfate proteoglycans on the cell surface. (5) Soluble viral interleukin ten (vIL-10) is secreted from the cell, binds to IL-10 receptors on inflammatory cells, blocking cell activation. (6) Soluble poxvirus interferon gamma receptor homologue (vIFN- $\gamma \mathrm{R}$ ) is also secreted from infected cells, binding and preventing host IFN- $\gamma$ from binding the cellular receptor. (7) Another poxvirus early protein that is responsible for immune modulation, soluble tumor necrosis factor receptor (vTNFR) homologue, binds host TNF and prevents its interaction with the cellular receptor. (8) Poxvirus interleukin eighteen binding protein (vIL-18BP) is secreted form the infected host cell and binds soluble IL-18, inhibiting its pro-inflammatory functions. (9) Orf virus encoded granulocyte-macrophage colony stimulating factor-inhibitory factor (GIF) is secreted and binds to granulocyte-macrophage colony-stimulating factor (GM-CSF), blocking its ability to stimulate the production and secretion of IFNQ. (10) Myxoma virus SERP-1, which is the only secreted poxvirus serpin, blocks several serine proteases, leading to reduction of inflammation. (11) The intracellular poxvirus serine protease inhibitor proteins, such as SPI-2, serp-2, and crmA, block the conversion of pre-caspase to active caspase one, thereby blocking its ability to convert pro-IL-1 $\beta$ to IL-1 $\beta$ and the cell's ability to undergo programmed cell death. 


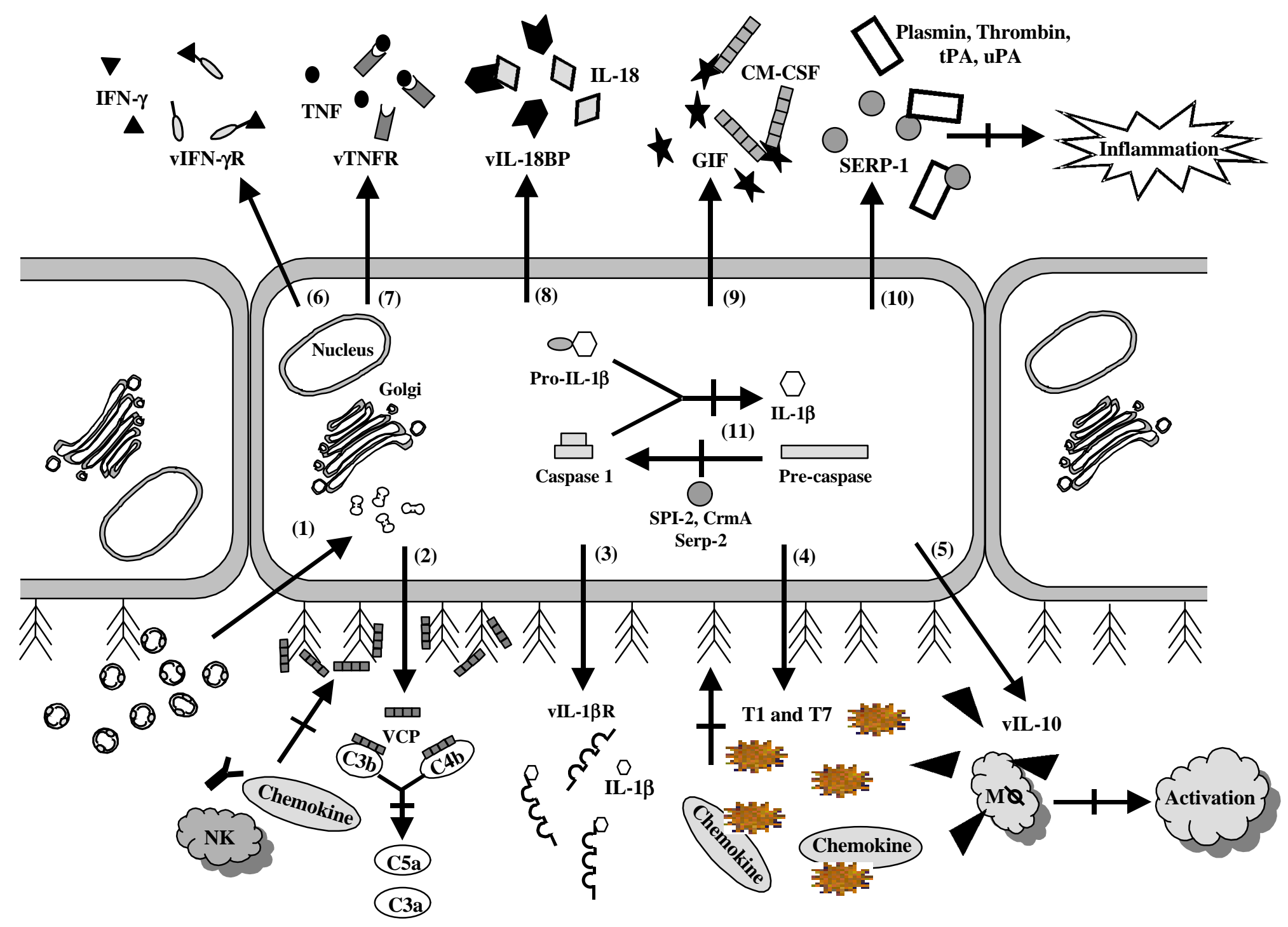


the viral infection, allowing the virus to replicate to high titers. Chemokine homologues and chemokine inhibitors (some of which are chemokine receptor homologues) were found to have a dramatic effect on leukocyte chemotaxis. These virokines have been shown to either help the virus evade immune detection, in some cases by blocking the influx of leukocytes into the area of infection, or in other cases, actually cause the recruitment of leukocytes to increase the pool of new host cells to allow for better dissemination. Chemokine inhibitors have been identified and shown to block cellular influx into the areas of infection. There are also virokines that block one of the most important aspects of the innate immune system, the complement system. These proteins are very powerful; they not only prevent the formation of the membrane attack complex (MAC), but also block the complement cascade at multiple sites upstream to block the formation of proinflammatory factors. They are therefore also able to greatly reduce the migration of leukocytes into the area of viral infection.

In addition to those proteins encoded by poxviruses that are secreted from the host cell, there are also proteins that act intracellularly. The most studied group of intracellular poxvirus immune modulating proteins are the serine proteinase inhibitors (serpins) (Kettle et al., 1997). Many poxviruses have been found to encode serpins that modulate programmed cell death, apoptosis. By blocking the cysteine proteinase interleukin-1 $\beta$-converting enzyme (ICE), and the serine proteinase granzyme B, the cowpox cytokine response modifier A (CrmA), vaccinia virus SPI-2, and myxoma virus Serp-2, all have been shown to inhibit apoptosis (Nash et al., 1999). By blocking these intracellular enzymes, the poxvirus serpins are able to prevent TNF- and IFN-induced, as well as T-lymphocyte-mediated, Fas-induced apoptosis (Tewari et al., 1995). By 
protecting the infected cell from programmed cell death, the virus is able to replicate to higher titers and continue host evasion.

\section{Complement Regulatory Proteins:}

Many poxviruses encode proteins that mimic components of the host complement system in order to block the activation of complement and thereby modulate the inflammatory response mounted by the host (Kotwal, 2000). See the section below for information on the vaccinia virus complement control protein, the best-studied poxvirus complement regulatory protein.

\section{Cytokine Homologues:}

The parapoxvirus, Orf virus, which often infects sheep and goats (sometimes humans), has very recently been shown to encode a viral homologue of IL-10 (vIL-10) (Fleming et al., 2000). This protein shows the greatest degree of homology, 80\%, to sheep IL-10. Endogenous IL-10 plays many important roles in down regulating the immune system. One very important function is to suppresses activation of macrophages, thereby blocking their ability to act as accessory cells to activate T-cells (especially $\mathrm{T}_{\mathrm{H}} 1$ ) or natural killer (NK) cells via IL-12 production and secrete cytokines. Thus, IL-10 acts as a potent anti-inflammatory agent and a powerful immunosuppressor. Because of this activity, it is believed that the Orf virus produces vIL-10 in order to inhibit expression of various cytokines that regulate the immune response against viral infection, suppressing activation of macrophages. The benefits of vIL-10 production for the virus are thereby to mask viral infection from the immune system long enough to replicate to high titers. 
Very recently, a new virokine, having homology to human interleukin-18 binding protein (hIL-18BP), was uncovered in molluscum contagiosum virus (MCV) (Xiang and Moss, 1999). Since this time, it has also been found in other poxviruses, including ectromelia, vaccinia, and cowpox (Smith et al., 2000). IL-18 is a proinflammatory cytokine important in activating natural killer (NK) cells, inducing a T-helper 1 response, and inducing the production of interferon gamma (IFN $\gamma$ ). The MCV vIL-18BP has been shown to bind to both human and murine IL-18 and inhibit the production of IFN $\gamma$ in a dose dependent manner (Xiang and Moss, 1999). Deletion of the gene encoding vIL18BP in VV was shown to dramatically attenuate the virus (Smith et al., 2000). In addition, the ectromelia virus vIL-18BP was shown to block IL-18 induced NF- $\mathrm{KB}$ activation and induction of IFN $\gamma$. This strategy of viral evasion therefore also acts to indirectly block the strong antiviral effects of IFN $\gamma$.

\section{Cytokine Receptor Homologues (Viroceptors):}

Many members of the poxvirus family, including myxoma, vaccinia, and cowpox, encode homologues of the tumor necrosis factor receptor (TNFR). This viral tumor necrosis factor receptor (vTNFR) was originally described in myxoma virus where it is known as T2, and in Shope fibroma viruses (SFV), and the term viroceptor was proposed to describe them (Upton et al., 1991). TNF is a very important cytokine for the early response to viral infection. It is a very powerful proinflammatory agent, triggering numerous immunological events, including apoptosis of virally infected cells. In order to block the effects of this powerful cytokine, these poxviruses have captured and modified the host gene for TNFR so that it may be secreted in large amounts from the infected cell. 
The vTNFR can bind soluble TNF present in the nearby area, preventing its binding to the cellular TNFR, thereby blocking the action of this cytokine. Many poxviruses encode several copies of this gene; cowpox virus, for example, contains three separate copies, showing its importance to viral replication (Loparev et al., 1998). In addition, when vTNFR (T2) was deleted from myxoma virus the virulence was significantly reduced. Interestingly, vTNFRs show a great deal of host specificity. Myxoma virus vTNFR, T2, will only bind to rabbit TNF (not at all to human), while the vTNFR encoded by vaccinia and cowpox viruses will bind human, mouse, and rat TNF effectively.

Many poxviruses, including variola, ectromelia, vaccinia, cowpox, and camelpox, also encode an additional interesting cytokine receptor homologue, viral IFN $\gamma$ receptor (vIFN $\gamma \mathrm{R})$. These proteins greatly resemble the N-terminal, ligand-binding (extracellular domain) region of their mammalian counterparts. As with vTNFR, these cytokine receptor homologues are also very species specific. The first and most extensively studied of these proteins comes from the myxoma virus and is known as T7 (Upton et al., 1992). T7 is a $37 \mathrm{kDa} \mathrm{N}$-glycosylated protein that is the most abundantly secreted protein of myxoma virus. Exhibiting great species specificity, T7 was shown to bind and block the effects of rabbit IFN $\gamma$, but had no effect in blocking human or murine IFN $\gamma$. In contrast, vaccinia virus, which has a broad host range, can bind and block a number of mammalian IFN $\gamma$ s. The importance of this encoded protein to myxoma virus virulence is evident, not only because it is the most abundantly secreted protein, but also because deletion of the gene eliminates the $99 \%$ mortality rate exhibited by the virus, and all infected rabbits recover. Therefore, the $\mathrm{T} 7$ protein represents the major virulence factor of myxoma virus. The importance of this protein to viral virulence is due to IFN $\gamma$ 's 
powerful antiviral effects. IFN $\gamma$ serves numerous functions, including switching on many intracellular antiviral activities, and giving the signal to nearby cells that a viral infection is occurring. Another cytokine receptor homologue, produced most notably by the orthopox members, vaccinia and cowpox viruses, is viral interleukin 1 receptor (vIL-1R). This $33 \mathrm{kDa}$ protein was first identified with vaccinia virus, and found to have the ability to bind murine IL-1 $\beta$ in 1992 (Alcami and Smith, 1992). A similar ORF was later identified in cowpox virus and the protein product was found to bind murine IL-1 $1 \beta$. Interestingly, supernatants taken from vaccinia virus-infected cells were found to inhibit IL-1 induced murine lymphocyte proliferation. In addition, recombinant vaccinia, unable to express vIL-1R, showed remarkable attenuation in vivo, compared to the wild type (Spriggs et al., 1992). This demonstrates how the protein plays a critical role in the pathology of vaccinia virus infection. The importance of IL-1 in combating viral infection is large, and was demonstrated many years ago (Vacheron et al., 1990). Expression of IL-1 is induced early on in many viral infections, and results in a broad spectrum of inflammatory responses. NK cells and B-cells proliferate in response to IL1, neutrophils degranulate, and T-cells produce IL-2 and proliferate.

Another very recently identified virokine was found encoded by the parapoxvirus, Orf (Deane et al., 2000). The Orf virus produces a secreted protein, termed granulocytemacrophage colony stimulating factor-inhibitory factor (GIF), which was shown to bind to and block the activity of GM-CSF, as well as IL-2. Both of these cytokines are very important in host antiviral immunity; IL-2 is essential for its role in the activation of Tcells, and GM-CSF for its ability to stimulate the production and secretion of IFN $\alpha$. This 
remarkable virokine plays a very novel role in modulation of host defense as a means of preserving viral habitat.

\section{Chemokine Homologues:}

Many poxviruses encode proteins that inhibit chemokines; the $\mathrm{CC}(\beta)$ chemokine homologue encoded by the molluscipoxvirus, molluscum contagiosum virus (MCV), designated as molluscum contagiosum chemokine homologue (MCCH) or MC148, was only recently discovered (Senkevich et al., 1996). This human disease-causing virus presumably utilizes this virokine to block leukocyte trafficking to the site of infection. MC148 is highly homologous to members of the $\mathrm{CC}$ or $\beta$ chemokine family; however, it is truncated in the $\mathrm{N}$-terminus of the protein. The $\mathrm{N}$-terminal portion of the chemokine is required for proper binding and activation of the cell (Senkevich et al., 1996). Therefore, the MC148 chemokine homologue of MCV acts by binding to the chemokine receptor, identified as being specifically CCR8, thereby blocking endogenous chemokine binding. The overall result of this protein is reduced cellular influx and therefore reduced inflammation. This protein seems to be geared toward specifically blocking monocyte infiltration and dendritic cell function.

\section{Chemokine Inhibitors:}

Many poxviruses encode a protein that blocks the effects of chemokines.

Collectively these virokines have been termed the T1/35kDa family, all of which possess eight conserved cysteine residues, and are shown to interact with members of the CC and/or CXC classes of chemokines (Graham et al., 1997). The myxoma virus T1 and T7 
proteins are the most widely studied members of this group. T1 shows no homology to any host chemokine or chemokine receptor and binds strongly (specifically) to members of the CC class of chemokines, but only weakly (nonspecifically) to CXC chemokines (Kettle et al., 1997). Because of this ability, T1 has been shown to block human monocyte migration, in the presence of MIP-1 $\alpha$, in vitro. Deletion of the coding sequence for $\mathrm{T} 1$ resulted in a pronounced increase in infiltrating monocytes and macrophages in the site of infection in vivo (Graham et al., 1997). However, the loss of T1 had no significant effect on mortality, indicating that this gene does not play a critical role in the pathogenesis of the infection. T7, on the other hand, has been shown to inhibit the activity of members of $\mathrm{C}, \mathrm{CC}$, and $\mathrm{CXC}$ classes of chemokines in a nonspecies specific manner. This protein, which is most noted for its anti-IFN $\gamma$ activity, has been shown to nonspecifically bind to the heparan sulfate proteoglycan binding domain of the chemokine, preventing gradient formation along the vascular wall, and inhibiting chemotaxis (Lalani et al., 1997). Addition of excess soluble heparin completely prevents this activity of the T7 protein, supporting this hypothesis. Since the T7 protein also exhibits no homology to any known chemokine, this strategy of chemokine inhibition is quite novel.

\section{Serine Protease Inhibitors:}

Several poxviruses have been shown to encode proteins that are able to prevent apoptosis, programmed cell death. The cowpox cytokine response modifier A (CrmA), vaccinia virus SPI-2, and myxoma virus Serp-2 have all been shown to inhibit apoptosis through the ability to block both, the cysteine proteinase interleukin- $1 \beta$-converting 
enzyme (ICE), and the serine proteinase granzyme B (Nash et al., 1999; Quan et al., 1995; Turner et al., 1999; Kettle et al., 1997; Turner et al., 1999). By blocking these intracellular enzymes, these poxvirus serine protease inhibitor (serpins) proteins are able to prevent TNF and IFN-induced, as well as T-lymphocyte-mediated $\left(\mathrm{Ca}^{+2}\right.$-independent), Fas-induced apoptosis (Nash et al., 1999; Tewari et al., 1995; Kettle et al., 1997). Therefore, these proteins prevent destruction of infected cells, even if they are recognized as being infected, thus maintaining a habitat conducive to virus replication.

Many members of the poxvirus family encode proteins that inhibit serine proteases intracellularly, but only myxoma virus encodes one that acts as a serine protease inhibitor (serpin) and is secreted extracellularly. First identified in 1990, this secreted glycoprotein, Serp-1, has structural features that place it in the serpin family of serine protease inhibitors (Upton et al., 1990). Before its discovery, intracellular serpins were found with VV (Kotwal and Moss, 1989). Serpins have many functions in maintaining homeostasis of eukaryotic systems, from complement activation and blood clotting, to the inflammatory response. In addition, mutations in serpins have been linked to over 90 human diseases. Given the importance of these proteins, many pathogens have evolved to carry their own serpins to disrupt host processes. Myxoma virus Serp-1, for example, has been shown in vivo to weaken the inflammatory response to viral infection. When this gene is deleted from the virus, a much greater inflammatory process is observed and the infection is resolved much more rapidly. Purified recombinant Serp-1 has been shown to bind a variety of proteases, including plasmin, thrombin, tissue-type plasminogen activator (tPA), and urokinase-type plasminogen activator (uPA), although the exact target has not been found (Lomas et al., 1993). This protein acts as a 
pseudosubstrate for these serine proteases, forming a stable complex, preventing the protease from binding its true substrate. Due to the role of Serp-1 in modulating inflammation, it was recently tested in a restenosis model system to evaluate its therapeutic value. 


\section{$\underline{\text { Vaccinia Virus Complement Control Protein (VCP) }}$}

The vaccinia virus complement control protein (VCP) was the first soluble microbial protein shown to have complement binding activity and postulated to have a role in evasion of host complement (Kotwal and Moss, 1988). In the years since its discovery, VCP has become one of the best studied and the best characterized virokine. Structurally, it is composed of four complement control protein (CCP) modules [also called short consensus repeats (SCR)], making it and other homologous proteins members of the regulators of complement activation (RCA) superfamily. VCP exhibits functional similarity to factor $\mathrm{H}(\mathrm{fH})$, membrane cofactor protein (MCP), decayaccelerating factor (DAF), complement receptor one (CR1), and is structurally most similar to human complement $4 \mathrm{~b}$ binding protein (C4b-BP). Purified bioactive VCP has been shown to inhibit both the classical and alternative pathways of complement activation through its ability to bind $\mathrm{C} 3$ and $\mathrm{C} 4$ and act as a cofactor for factor I cleavage of C3b and C4b (Sahu et al., 1998). VCP, secreted from virally infected cells, is believed to protect the cell and the released viral progeny from host complement attack. In addition, by blocking complement activation at multiple sites, there is a large reduction of C3a, C4a, and C5a proinflammatory chemotactic factors, resulting in reduced cellular influx and inflammation. VCP has also been shown to possess the ability to bind heparin, as well as, heparan sulfate proteoglycans (heparin-like molecules) on the surface of human endothelial cells. Because of this activity, VCP has been shown to exhibit many properties that all other complement control proteins are lacking. In vivo studies have indicated that FITC-labeled VCP, when injected intravenously, becomes localized in the endothelium. This very interesting property of VCP has been shown in vitro to reduce 
chemotactic migration of leukocytes in the presence and absence of the chemokine MIP$1 \alpha$ (Reynolds et al., 2000). This suggested that VCP could interfere with molecular interactions with infected cells, and possibly prevent antibody-dependent cell-mediated cytotoxicity (ADCC) as well as other cytotoxic cell interactions with target cells. Amazingly, VCP was shown to block interactions between pig aortic endothelial cells (PAECs) and naïve neutrophils and NK cells, preventing cell killing (Al-Mohanna et al., 2001). In addition, cell culture studies demonstrate that VCP blocks complementmediated killing of PAECs by human serum, in a dose-dependent manner. VCP is further discussed in chapters 2-6. 


\section{CHAPTER 2}

\section{EXPRESSION, PURIFICATION, AND CHARACTERIZATION OF VCP}

\section{$\underline{\text { VCP from VV Infection of Mammalian Cell Culture }}$}

This chapter contains material adapted from the following publication:

Smith S.A., N.P. Mullin, J. Parkinson, S.N. Shchelkunov, A.V. Totmenin, V.N. Loparev, R. Srisatjaluk, D.N. Reynolds, K.L. Keeling, D.E. Justus, P.N. Barlow, and G.J. Kotwal. Conserved surface-exposed K/R-X-K/R motifs and net positive charge on poxvirus complement control proteins serve as putative heparin binding sites and contribute to inhibition of molecular interactions with human endothelial cells: a novel mechanism for evasion of host defense. J. Virol. Jun. 74(12):5659-66, 2000 [Cover of J. Virol. Oct. 74(20), 2000].

Natural VCP, produced by VV during a natural infection process of cultured cells, was produced in large quantities with technical assistance. Briefly, RK-13 cells were grown to confluency in large cell factories before infecting with VV WR strain. After 48 hours the media was harvested, filtered through a $0.22 \mu \mathrm{m}$ filter, and concentrated to $\sim 50$ ml using an Amicon stirred-cell (with $10 \mathrm{kDa}$ molecular size cut-off). Heparin HiTRAP (Pharmacia) chromatography was then performed on the media for specific purification of VCP. A NaCl step gradient (100 mM - 1 M) was used to rinse and elute VCP from the column. Finally, the fractions were examined by SDS-PAGE and Coomassie blue staining. VCP eluted from the heparin column and was primarily located in the $400 \mathrm{mM}$ $\mathrm{NaCl}$ elution fraction. All fractions containing VCP were then pooled, the protein concentration was estimated using a Bio-Rad protein assay kit, and the protein's activity 
estimated using the hemolysis assay (described in chapter 4) (Kotwal and Moss, 1988). Natural VCP was frequently isolated at greater than 90\% purity (using SDS-PAGE analysis) by this method. 


\section{Pichia pastoris Yeast Expression System}

Nick P. Mullin from the Edinburgh Center for Protein Technology, University of Edinburgh, UK expressed VCP and fragments of VCP in Pichia pastoris using the secretory expression vector pPIC9, see figure 6 . The fragments corresponded to amino acids 18-146 (rVCP 1,2), 82-204 (rVCP 2,3), 145-263 (rVCP 3,4) and 18-262 (rVCP 1-4). Genomic DNA from vaccinia virus was used as template for the amplification of the DNA fragments encoding the above protein fragments by PCR. In all cases except for VCP 3,4 the oligonucleotides used introduced a 5' Eco RI site and a 3' Not I site which were used for cloning the fragments into the expression vector. For VCP 3,4, cloning was carried out as described earlier (Wiles et al., 1997).

Selection of clones and expression of $\mathrm{rVCP} \sim 3,4$ has been described previously (Wiles et al., 1997). For rVCP 1,2, rVCP 2,3 and rVCP 1-4 clones transformed with the expression vectors described above where selected on the basis of their ability to grow on histidine deficient medium. Small-scale expression screening was performed by inoculating $5 \mathrm{ml}$ of buffered minimal glycerol (BMG) and incubating at $30{ }^{\circ} \mathrm{C}$ until the $\mathrm{OD}_{600}$ reached between 6 and 10. The cells were harvested and resuspended in $2 \mathrm{ml}$ (for KM71 cells) or $15 \mathrm{ml}$ (for GS115 cells) of buffered minimal methanol (BMM). Cells were grown with vigorous shaking for 4 to 5 days with daily addition of methanol to reach a concentration of $0.5 \%$ [for activation of gene expression (see figure 5)]. Media from these inductions was analysed by SDS-PAGE and the highest-level expressers selected. For $\mathrm{rVCP} \sim 1,2, \sim 2,3$, and $\sim 1-4$, the KM71 cell line proved to produce the most protein. For large-scale growth of $\mathrm{rVCP} 1,2$ and $\mathrm{rVCP} \sim 1-4,100 \mathrm{ml}$ of $\mathrm{BMG}$ was inoculated with $5 \mathrm{ml}$ of an overnight culture and grown at $30^{\circ} \mathrm{C}$ with vigorous shaking 
Figure 6. Pichia pastoris yeast expression system. Listed are the steps used for cloning and growth using the Pichia pastoris yeast expression system by Invitrogen. Also shown, is the pPIC9 expression vector used in the cloning of VCP and the various VCP truncations. Special features of the pPIC9 vector are depicted in the diagram. 


\section{|III/ Invitrogen}

1) Select the appropriate expression vector.

2) Clone your desired gene into vector.

3) Transform E. coli, select ampicillin-resistant transformants, and confirm presence and orientation of desired gene.

4) Linearize constructs with appropriate restriction enzymes to generate $\mathrm{His}^{+} \mathrm{Mut}^{\mathrm{s}}$ recombinant strain.

5) Transform and select for $\mathrm{His}^{+}$transformants.

6) Screen $\mathrm{His}^{+}$transformants for $\mathrm{His}^{+} \mathrm{Mut}^{\mathrm{s}}$ strains

7) Confirm integration of your gene in $\mathrm{His}^{+} \mathrm{Mut}^{\mathrm{s}}$ strain by PCR.

8) Grow recombinants in $\mathbf{1 0 0 - 2 0 0} \mathrm{ml}$ of buffered glycerol medium (BMGY) to an OD $_{600}=2-6$.

9) Harvest cells and resuspend to an $\mathrm{OD}_{600}$ of 10 with methanol medium (BMMY) and place in $250 \mathrm{ml}$ flask.

10) Incubate at $30^{\circ} \mathrm{C}$ with shaking and take samples for analysis at $0,24,48,72,96,120$, and 144 hours.

11) Analyze medium for protein via PAGE/Coomassie Blue staining, western blot, activity, ELISA, or immunoprecipitation.

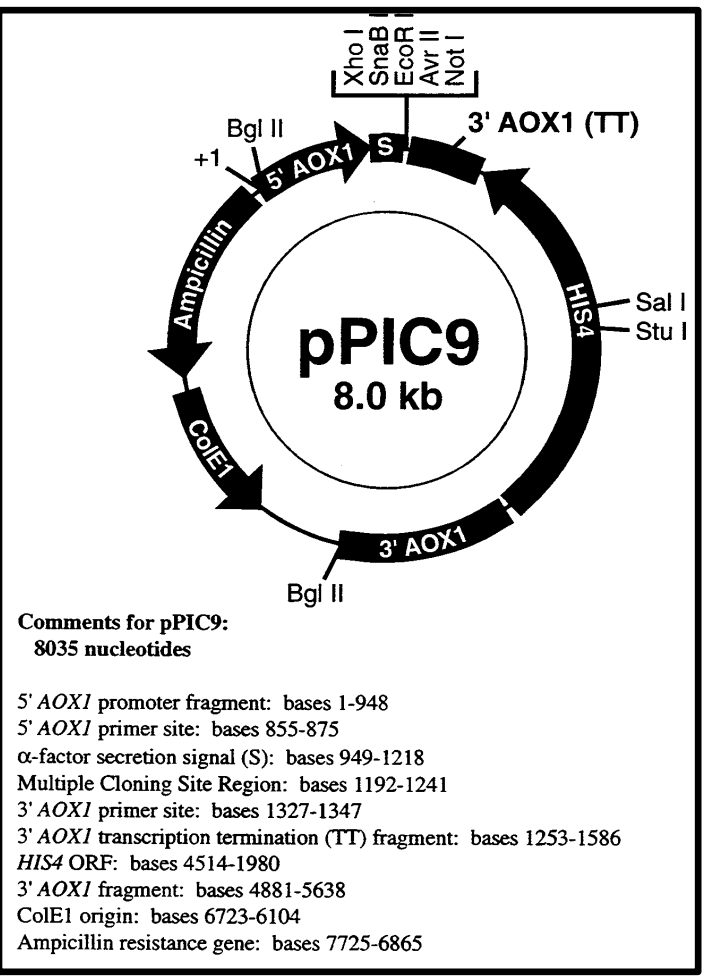


overnight. This culture was then used to inoculate several liters of BMG. The cultures were grown for 48 hours until the OD reached approximately 20. The cells were spun down, resuspended in the same volume of BMM, and grown for 4 to 5 days with vigorous shaking. Cells were fed methanol to a concentration of $1 \%$ every 24 hours. For rVCP 2,3, BMG media was inoculated with an overnight culture and grown at $30^{\circ} \mathrm{C}$ with vigorous shaking until an OD of approximately 6 had been attained. The pellet was then harvested by centrifugation, resuspended in BMM media (40\% of the total BMG volume) and grown for 5 days with daily addition of methanol to a concentration of $0.5 \%$.

Purification of rVCP 3,4 has been described previously (Wiles et al., 1997). For purification of $\mathrm{rVCP} 1,2$, media was concentrated down to a small volume using a combination of a Millipore Prep/Scale-TFF cartridge (3 kDa molecular size cut-off) and Amicon stirred-cell (with $3 \mathrm{kDa}$ molecular size cut-off). The sample was exchanged into 50 mM Tris- $\mathrm{HCl}(\mathrm{pH}$ 9.0) using a Pharmacia PD-10 column and loaded onto a Pharmacia Mono-Q column, equilibrated in the same buffer. The protein was then eluted with a $\mathrm{NaCl}$ gradient of 0 to $100 \%$ over twenty minutes. Fractions containing $\mathrm{rVCP} 1,2$ were collected and concentrated using Amicon stirred-cell ultrafiltration and then loaded onto a Brownlee Aquapore C4 reverse-phase column and the protein eluted with an acetonitrile gradient. Fractions containing pure rVCP 1,2 were collected and lyophilized. For purification of $\mathrm{rVCP} 2,3$, media was concentrated to approximately 50 $\mathrm{ml}$ using an Amicon concentrator (3 kDa cut-off) before being centrifuged at 20,000 x g for 1 hour and the pellet discarded. The sample was exchanged into $5 \mathrm{mM}$ sodium acetate ( $\mathrm{pH} 4.0)$ using a PD-10 column and applied to a Mono-S cation exchange column (Amersham Pharmacia, Uppsala, Sweden), equilibrated in the same buffer. Protein was 
eluted with a $\mathrm{NaCl}$ gradient and fractions corresponding to pure $\mathrm{rVCP} 2,3$ were pooled and lyophilized. For purification of $\mathrm{rVCP} \sim 1-4$, media was concentrated to a small volume by the same means as $\mathrm{rVCP} 1,2$. The protein was exchanged into $20 \mathrm{mM}$ phosphate $\mathrm{pH} 6$ using a PD-10 column and dried. 


\section{Growth and Affinity Purification}

$\underline{B M G Y-\text { versus- BMMY: }}$

The various rVCP yeast clones described previously were stored in $10 \%$ glycerol and frozen to $-80^{\circ} \mathrm{C}$. When growth is desired, the frozen stock was plated on minimal methanol histidine media $(\mathrm{MMH})$ for selecting and maintaining a pure culture of the clone. Optimal growth on $\mathrm{MMH}$ was achieved through incubation at $30^{\circ} \mathrm{C}$ for several weeks. A single colony was then used for inoculation of starter culture.

Using sterile technique, a single colony was removed from the MMH plate and inoculated into $1 \mathrm{ml}$ buffered minimal-complex glycerol medium (BMGY). One liter of BMGY consists of: $750 \mathrm{ml}$ peptide base (30 g YEP in one liter $\mathrm{H}_{2} \mathrm{O}$, autoclaved), $100 \mathrm{ml}$ 10X glycerol (100 ml glycerol in $900 \mathrm{ml} \mathrm{H}_{2} \mathrm{O}$, autoclaved), $100 \mathrm{ml}$ phosphate buffer $\mathrm{pH}$ 6.0 ( $\sim 132 \mathrm{ml} 1 \mathrm{M} \mathrm{K}_{2} \mathrm{HPO}_{4}$ and $\sim 8681 \mathrm{M} \mathrm{KH}_{2} \mathrm{PO}_{4}$, autoclaved), 50 ml yeast nitrogen base (1.7 $\mathrm{g}$ YNB in $100 \mathrm{ml} \mathrm{H}_{2} \mathrm{O}$, sterile filtered), and $2 \mathrm{ml} 500 \mathrm{X}$ biotin (sterile filtered). The $1 \mathrm{ml}$ starter culture was grown at $30^{\circ} \mathrm{C}$ overnight. The starter culture was then used to inoculate the remaining $999 \mathrm{ml}$ BMGY in a large 4 L Erlenmeyer flask, the mouth covered with cheesecloth. The culture was then incubated at $30^{\circ} \mathrm{C}$ with vigorous shaking (200 rpm) for 2-3 days. The cells were then removed by swing bucket centrifugation (3,000 rpm) for $30 \mathrm{~min}$ using $250 \mathrm{ml}$ polypropylene bottles. Being careful to prevent contamination, the cells were then resuspended in $500 \mathrm{ml}$ (50\% of the total BMGY volume) buffered minimal-complex methanol medium (BMMY). BMMY consists of: $250 \mathrm{ml}$ peptide base (30 g YEP in one liter $\mathrm{H}_{2} \mathrm{O}$, autoclaved), $500 \mathrm{ml}$ autoclaved $\mathrm{H}_{2} \mathrm{O}$, $100 \mathrm{ml} 20 \%$ methanol (200 ml methanol in $800 \mathrm{ml} \mathrm{H}_{2} \mathrm{O}$, autoclaved), $100 \mathrm{ml}$ phosphate buffer pH 6.0 ( $\sim 132 \mathrm{ml} 1 \mathrm{M} \mathrm{K}_{2} \mathrm{HPO}_{4}$ and $\sim 8681 \mathrm{M} \mathrm{KH}_{2} \mathrm{PO}_{4}$, autoclaved), $50 \mathrm{ml}$ yeast 
nitrogen base (1.7 $\mathrm{g} \mathrm{YNB}$ in $100 \mathrm{ml} \mathrm{H}_{2} \mathrm{O}$, sterile filtered), and $2 \mathrm{ml} 500 \mathrm{X}$ biotin (sterile filtered). The culture was then incubated at $30^{\circ} \mathrm{C}$ with vigorous shaking (200 rpm) for 2 days. Additional methanol was added to a final concentration of $2 \%(10 \mathrm{ml})$ after the first day. After two days of incubation, the cells were then removed by high-speed centrifugation (12,000 rpm) for $45 \mathrm{~min}$ using $250 \mathrm{ml}$ polycarbonate bottles. The media was then carefully decanted and passed through a $0.22 \mu \mathrm{m}$ bottle-top filter. The media was then concentrated using an Amicon stirred-cell with $10 \mathrm{kDa}$ molecular weight cut-off membrane to $\sim 50 \mathrm{ml}$.

\section{Affinity Purification and Endotoxin Removal:}

Recombinant VCP $(\mathrm{rVCP}=\mathrm{rVCP} \sim 1-4)$ was purified from the concentrated BMMY media by using heparin HiTRAP (Pharmacia) affinity chromatography. BMMY was first pumped at a rate of $1 \mathrm{ml} / \mathrm{min}$ through a sterile filter $(0.22 \mu \mathrm{m}$ syringe filter $)$ into a series of five heparin HiTRAP columns run in tandem. The unbound material was collected and the column was washed with $50 \mathrm{ml}$ sterile $\mathrm{H}_{2} \mathrm{O}$. A NaCl $30 \mathrm{ml}$ step gradient ranging from $250 \mathrm{mM}$ to $550 \mathrm{mM}$, increasing in $50 \mathrm{mM}$ increments, was then applied to the column to elute the bound protein. Finally, the column was washed with $50 \mathrm{ml} 2 \mathrm{M} \mathrm{NaCl}$ to remove any remaining proteins. The fractions were then visualized by SDS-PAGE and coomassie blue staining, see figure 7. The fractions containing rVCP were then pooled, the protein concentration was estimated using a Bio-Rad protein assay kit, and the protein's activity estimated using the hemolysis assay (Kotwal and Moss, 1988). The purity of the recovered rVCP was frequently greater than $95 \%$ by this 
Figure 7. Heparin purification of $\mathbf{r V C P}(\mathbf{r V C P} \sim 1-4)$. Coomassie blue stained SDSPAGE gel shows fractions following heparin affinity chromatography. Fractions shown are from a $\mathrm{NaCl}$ elution step gradient ranging from $250 \mathrm{mM}$ to $550 \mathrm{mM}$ (by $50 \mathrm{mM}$ increments) and a $2 \mathrm{M}$ wash. S.M. = starting material, Unb. = unbound fraction. 


\section{Heparin Purification of rVCP}

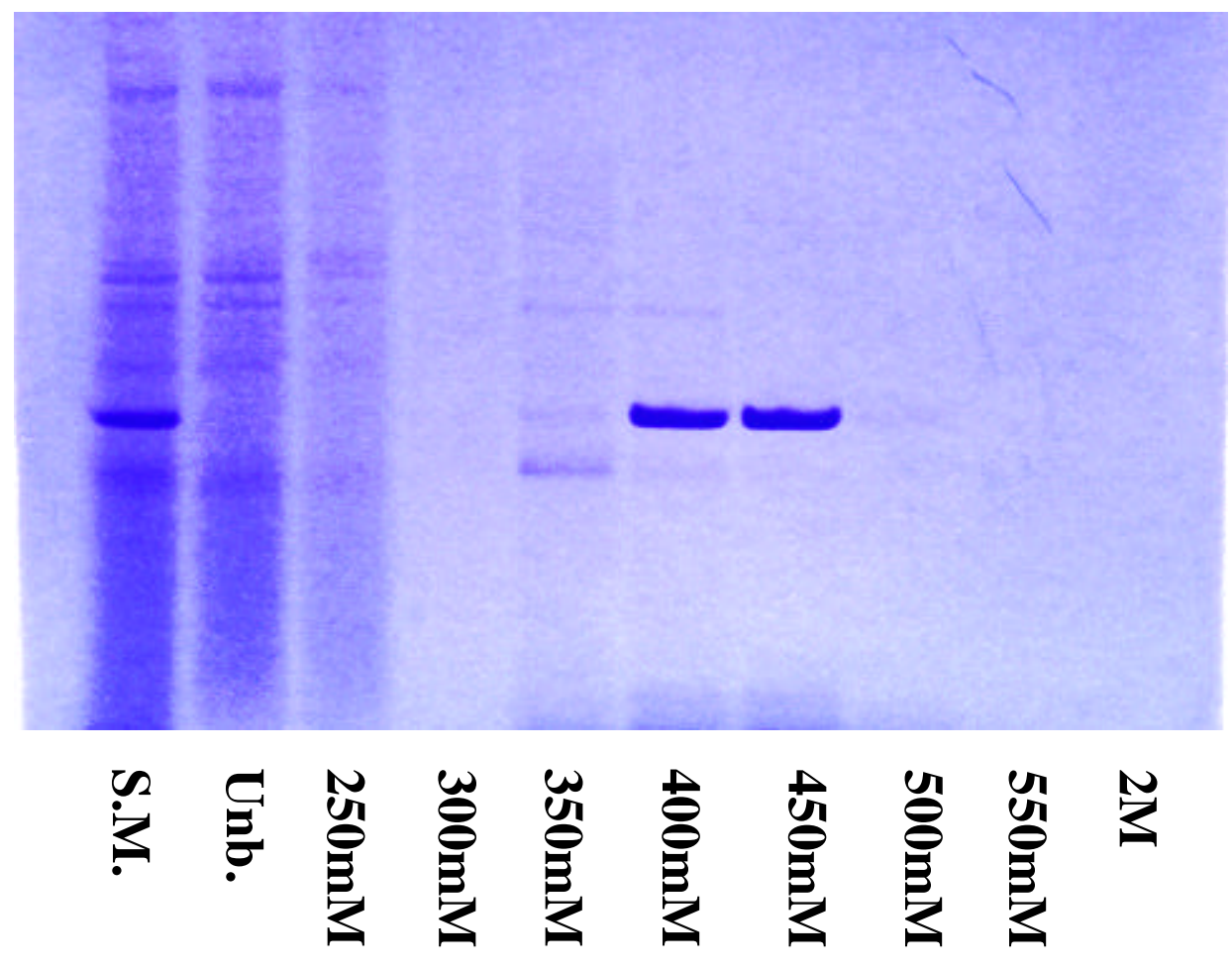


method. This procedure can be used for purification of $\operatorname{rVCP} 1-4, \operatorname{rVCP} \sim 1,2, \operatorname{rVCP} \sim 3,4$, and $\mathrm{rVCP} \sim 2,3,4$, but cannot be used to purify $\mathrm{rVCP} 2,3$.

rVCP 2,3 was purified most effectively by affinity chromatography using HiTRAP ANX (high sub) sepharose fast flow (Pharmacia). ANX sepharose is a weak anion exchanger, similar to DEAE, with a high degree of cross-linking. This allows for better purification of $\operatorname{rVCP} 2,3$ and higher yields. Similar methodology was used for chromatographic purification of $\mathrm{rVCP} \sim 2,3$ as compared to the other $\mathrm{rVCP}$ clones, with the exception of the step gradient. $\mathrm{A} \mathrm{NaCl}$ step gradient ranging from $50 \mathrm{mM}$ to 350 $\mathrm{mM}$, increasing in $50 \mathrm{mM}$ increments, was applied to the column to elute the bound protein. Visualization and characterization of the protein was the same as above.

If contamination of the yeast culture does not occur, samples of rVCP will contain only trace amounts of endotoxin and do not need further purification. However, if contamination occurs, and endotoxin is present (BioWhittaker Limulus Amebocyte Lysate endotoxin detection assay), endotoxin removal can be carried out as follows. Twenty milliliters of endotoxin removal gel, polymyxin B sepharose, was seeded into a $30 \mathrm{ml}$ all-purpose HPLC column, washed with endotoxin free water, and activated using the appropriate activation buffer. Keeping in mind that the matrix has an endotoxin binding capacity of 5,000 EU/ml, the appropriate amount of $\mathrm{rVCP}$ was then passed through the column at a $0.5 \mathrm{ml} / \mathrm{min}$ flow rate. The column was then rinsed with a column volume of endotoxin free water and a $\mathrm{NaCl}$ gradient $\left(\mathrm{H}_{2} \mathrm{O}\right.$ to $\left.1 \mathrm{M}\right)$ was used to elute the rVCP that was nonspecifically bound. Fraction collection was continually monitored at $280 \mathrm{~nm}$, and two rVCP peaks are visible; the first peak was rVCP which flowed directly through the matrix, and the second was rVCP which had a nonspecific interaction with 
the matrix and was eluted with low $\mathrm{NaCl}$, see figure 8 . Fractions were then visualized by SDS-PAGE and coomassie blue staining, the fractions were pooled, and the protein concentration estimated using a Bio-Rad protein assay kit. Finally, the final concentration of endotoxin was estimated using the BioWhittaker Limulus Amebocyte Lysate endotoxin detection assay and compared with the original estimate to determine the endotoxin reduction, see figure 9. 
Figure 8. Endotoxin removal using Polymyxin B HPLC. A representative graph of the $280 \mathrm{~nm}$ scan and fractions following endotoxin removal of rVCP using Polymixin B chromatography is shown. The $280 \mathrm{~nm}$ scan is shown in blue, the $\mathrm{NaCl}$ gradient shown in red, and peaks one and two are labeled. rVCP elutes from the column as two peaks because of a low affinity which some rVCP exhibits toward Polymixin B. 


\section{Polymyxin B Gel Chromatography}

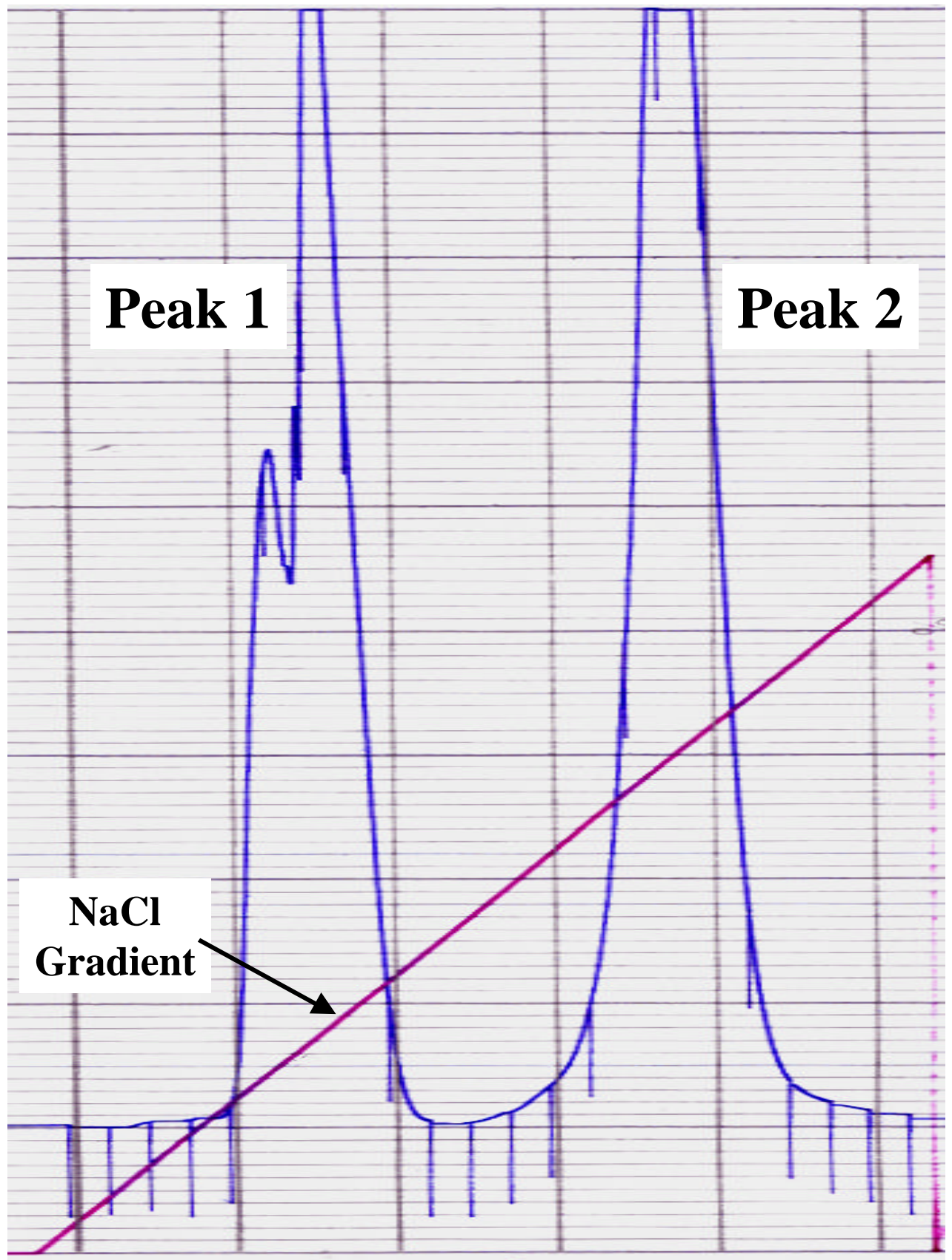


Figure 9. Endotoxin removal of rVCP. The concentration of endotoxin in a sample of rVCP is shown before and after each step of purification. Heparin affinity chromatography was performed as described in the "Growth and Affinity Purification" section of chapter 2, protein concentrations were estimated using the Bio-Rad protein assay kit, and endotoxin was estimated using the BioWhittaker Limulus Amebocyte Lysate endotoxin detection assay. 


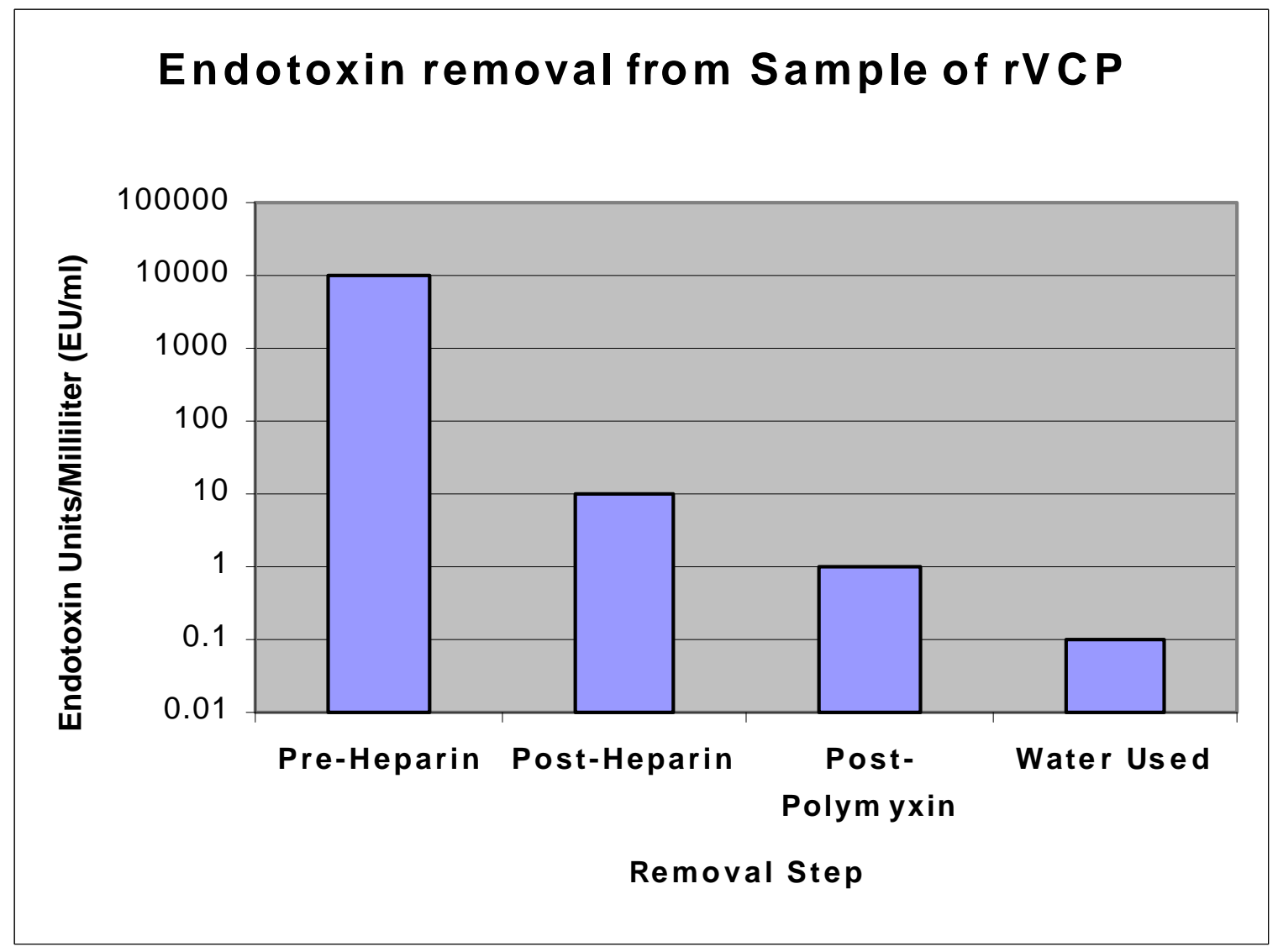




\section{Assays and Characterization}

\section{Hemolysis Assay and Activity Units:}

The hemolysis assay was developed to test the activity of VCP quickly and easily using a microtiter plate reader (Kotwal and Moss, 1988). At the heart of the assay is the sensitized sheep red blood cell (sSRBC) (Diamedix). In the presence of serum, the classical complement pathway will be activated on the surface of the erythrocyte, resulting in its lysis. In the presence of $\mathrm{VCP}$, however, the complement pathway activation will be inhibited and the erythrocyte will not be lysed. The assay was performed in $0.65 \mathrm{ml}$ general-purpose polypropylene Eppendorf tubes on ice, see figure 10 for diagram. First, half of the sSRBC buffer $(1.5 \mathrm{ml})$ was removed to obtain the correct concentration for the assay (OD of $5.0 @ 405 \mathrm{~nm}$ ). The VCP sample was then serially diluted in $20 \mu \mathrm{l}$ of the buffer. Next, $30 \mu \mathrm{l}$ of diluted serum ( 1:70) was added (must be able to only lyse $95 \%$ of the sSRBCs). Finally, $150 \mu 1$ of sSRBCs was added to each tube to give a final volume of $200 \mu \mathrm{l}$. A positive control was made by adding no $\mathrm{VCP}$, and a negative control was made by adding no serum. The tubes are incubated for one hour at $37^{\circ} \mathrm{C}$, and then centrifuged at $7,000 \mathrm{rpm}$ for 20 seconds. Directly after centrifugation, $150 \mu \mathrm{l}$ of the supernatant was then pipetted into a 96 well flat-bottomed microtiter plate. The plate was then read at $405 \mathrm{~nm}$, and the activity units are derived from the percent lysis inhibition which was calculated using the formula \{[(O.D. sample - O.D. negative control)/(O.D. positive control - O.D. negative control) X 100] - 100\}. Specific activity for VCP can be determined using the hemolysis assay. Specific activity units are defined as the quantity of VCP required to inhibit hemolysis to $50 \%$ in the hemolysis assay. To determine the total activity units of the VCP sample, the 
Figure 10. Hemolysis assay. Illustrated are the procedures used to perform the hemolysis assay to measure the activity of VCP. After serially diluting the sample of VCP, enough serum is added to lyse $95 \%$ of the sSRBSs. Lastly, the sSRBCs are added and the tubes are incubated at $37{ }^{\circ} \mathrm{C}$ for $1 \mathrm{~h}$. After incubation, the tubes are centrifuged for $20 \mathrm{sec}$ and the supernatant pipetted into a 96 well flat-bottomed microtiter plate. The absorbance is read at $405 \mathrm{~nm}$ using a microtiter plate reader $\left(\mathrm{EL}_{X} 800\right.$ BIO-TEK Instruments, Inc.) and the activity of the sample is calculated, as indicated in the "Assay and Characterization" section of chapter 2. 


\section{Hemolysis Assay}

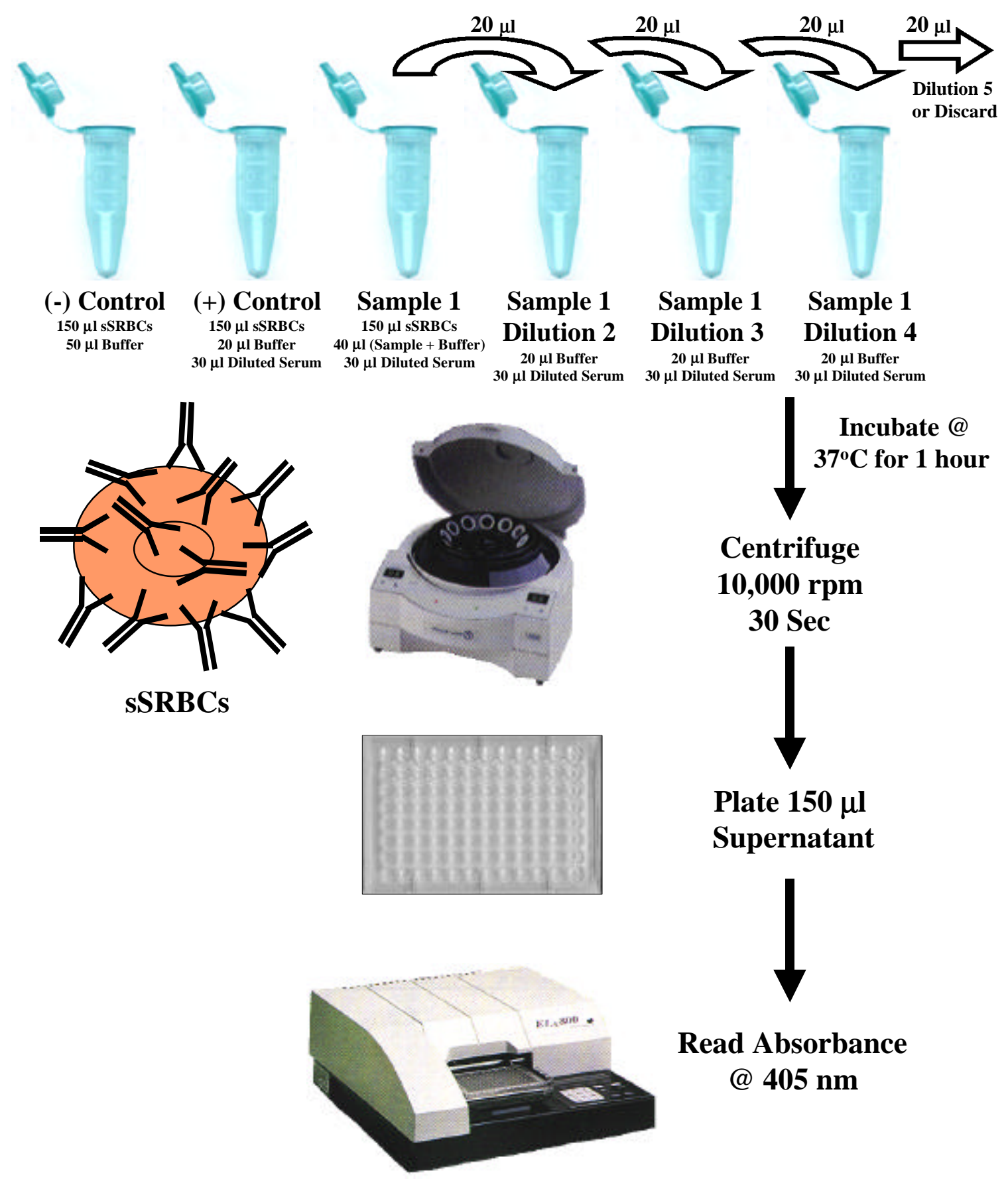


appropriate dilution (producing 50\% lysis inhibition) is multiplied by the total volume of the sample. In most cases, one activity unit equates to approximately $0.25-0.50 \mu \mathrm{g}$ of VCP.

\section{Protein Estimation Assay:}

Accurate estimation of the amount of purified rVCP and rVCP fragments can be done quickly and easily using the Blue Bio-Rad protein assay. Bovine serum albumin was used as a standard for accurate concentration estimates. A microtiter plate reader was used for this assay, which reduces the quantity of protein required (5 $\mu \mathrm{l})$ to do the estimation. This was far superior to the Red Bio-Rad protein assay, which uses a spectrophotometer and $100 \mu 1$ of sample. 


\section{VCP Production for NMR, X-Ray Crystallography, and Antibodies}

\section{Labeling for NMR:}

In order to obtain an NMR structure of VCP, all amino acids of the protein must be labeled with $\mathrm{N}^{15}$ non-radioactive isotope. Typically, the best way to achieve this is by growing the yeast in the presence of $\mathrm{N}^{15}$ labeled ammonium sulfate. Due to the great expense of this labeled compound, optimal growth and labeling conditions were first established using unlabeled ammonium sulfate. Yeast were first grown under normal conditions in $500 \mathrm{ml} \mathrm{BMGY}$, as described above. After removing the medium by centrifugation, the yeast were starved for 6 hours in ammonium sulfate media. This media consisted of the following: $2.5 \mathrm{~g}$ ammonium sulfate, $225 \mathrm{ml}$ sterile $\mathrm{H}_{2} \mathrm{O}$, and $25 \mathrm{ml}$ 10X glycerol. This medium is designed to replace the unlabeled amino acid reserves in the yeast with what would normally be $\mathrm{N}^{15}$ labeled amino acids, from the ammonium sulfate. As a control, the same experiment was done without ammonium sulfate, to test the turnover of the amino acid reserves, see figure 11A. Finally, the yeast were growth for 3 days, with daily addition of methanol (to a final concentration of $2 \%$ ), in ammonium sulfate methanol medium. This media consisted of the following: $2.5 \mathrm{~g}$ ammonium sulfate, $225 \mathrm{ml}$ sterile $\mathrm{H}_{2} \mathrm{O}$, and $25 \mathrm{ml}$ of $10 \%$ methanol. After removing the yeast by high-speed centrifugation, the media was filtered and the protein was purified by heparin affinity chromatography, see figure 11B. Yeast grown without ammonium sulfate produced only a small amount of protein (no nitrogen source), indicating that the vast majority of $\mathrm{rVCP}$ produced by yeast growing in the presence of ammonium sulfate was the result of this compound being present. The fractions containing rVCP were then pooled and the protein concentration was estimated. Using this technique, $1 \mathrm{mg}$ of 
Figure 11. Heparin affinity chromatography of ammonium sulfate rVCP. In order to determine the amount of rVCP that can be produced, for use in NMR, by growing the Pichia pastoris yeast clone in minimal media, yeast were grown by two methods, with and without ammonium sulfate. A) rVCP produced after heparin chromatography following growth in minimal media containing no ammonium sulfate. B) rVCP produced after heparin chromatography following growth in minimal media containing ammonium sulfate. In both cases, $\mathrm{rVCP}$ was purified using a $\mathrm{NaCl}$ elution step gradient ranging from $100 \mathrm{mM}$ to $500 \mathrm{mM}$ (increasing increments of $50 \mathrm{mM}$ ). The starting material, unbound material and rVCP marker are also shown. 


\section{Heparin Purification of Ammonium Sulfate rVCP}

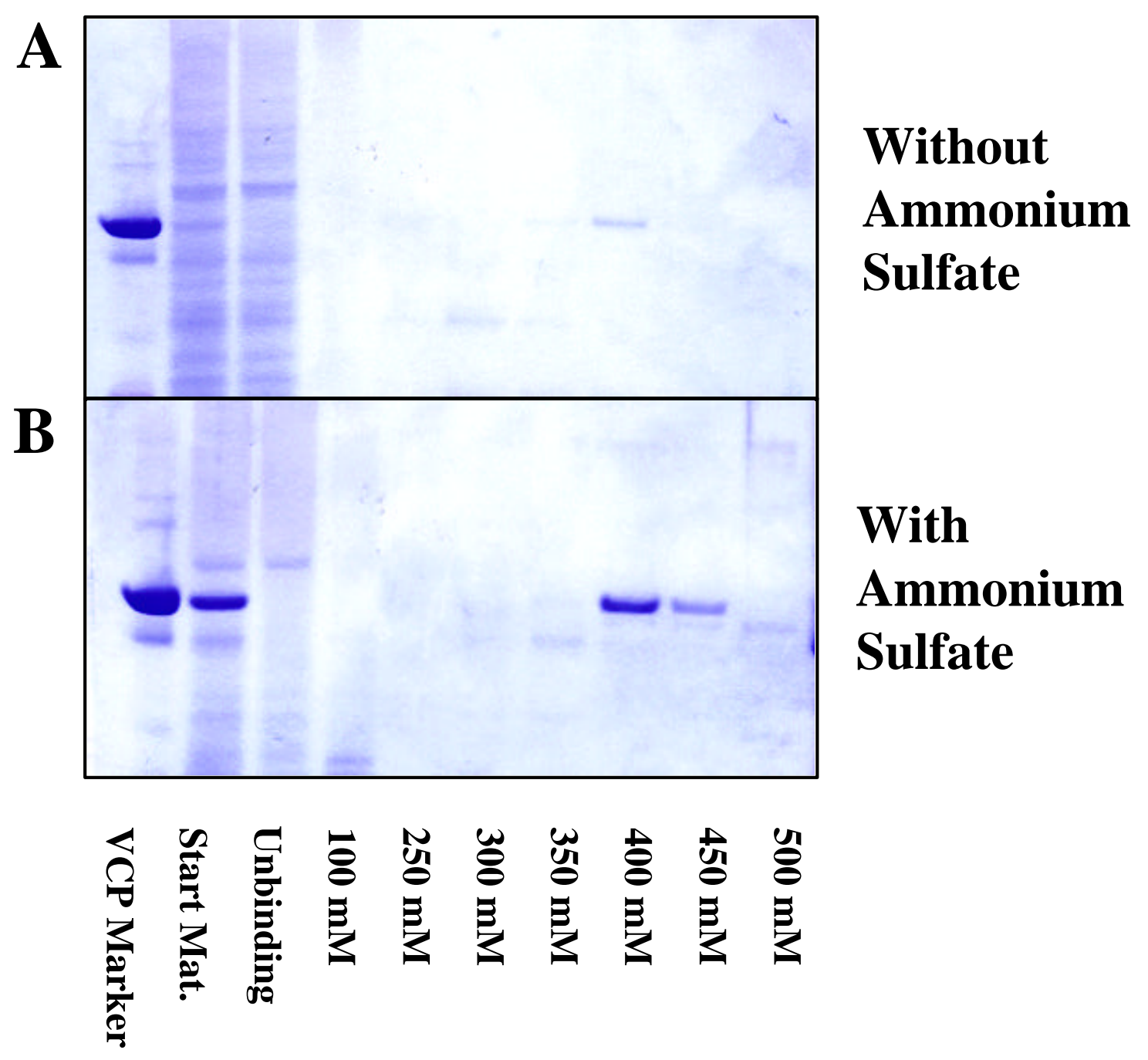


greater than $95 \%$ pure rVCP can be made using $5 \mathrm{~g}$ of ammonium sulfate. Due to the great cost required, $\mathrm{N}^{15}$ labeled ammonium sulfate was never used.

\section{Large Scale Purification for X-Ray Crystallography:}

In order to produce large quantities of highly purified rVCP for X-ray crystallography, the above procedure was scaled up to 2 liters. After heparin purification was repeated a second time, the purity of rVCP was found to be approximately $98 \%$, see

figure 12 . The protein concentration was estimated to be approximately $12.5 \mathrm{mg}$, and the activity units estimated to be approximately $5 \times 10^{5}$. The sample was then sent for crystallization as $10 \%$ glycerol at $4^{\circ} \mathrm{C}$. Crystals were produced and the structure of $\mathrm{rVCP}$ was obtained, discussed in chapters 3-5.

\section{Anti-VCP Production, Purification, and Characterization:}

In order to obtain antibodies to rVCP, for use in immunohistochemisty and Western blot analysis, purified protein was mailed to Washington Biotec (Baltimore, Maryland) for immunization of a chicken and a rabbit. Both the rabbit and chicken received three immunizations. In order to obtain antibodies to linearized epitopes on rVCP to be used for Western blotting, the second immunization given to the rabbit was of denatured rVCP. After running several milligrams of rVCP on SDS-PAGE with reducing agent, the protein was purified by gel extraction. Immunization of the chicken and purification of $\operatorname{IgY}$ from hen eggs was done by Washington Biotech, Inc. Due to the high concentration of non-specific antibodies in the samples, further purification of antirVCP antibodies was desired. Purification was achieved using a Peirce Amino-link Plus 
Figure 12. Purity of rVCP used in X-Ray crystallography. Various amounts of purified rVCP are shown by SDS-PAGE and Coomassie blue staining. Also shown is the total volume, number of milligrams, and total activity units of the sample. This sample contained $10 \%$ glycerol (total volume) and was sent to Dr. Krishna Murthy for crystallization studies, which ultimately resulted in the crystal structure of rVCP, described in chapters 3-5. 


\section{rVCP for X-ray Crystallography}

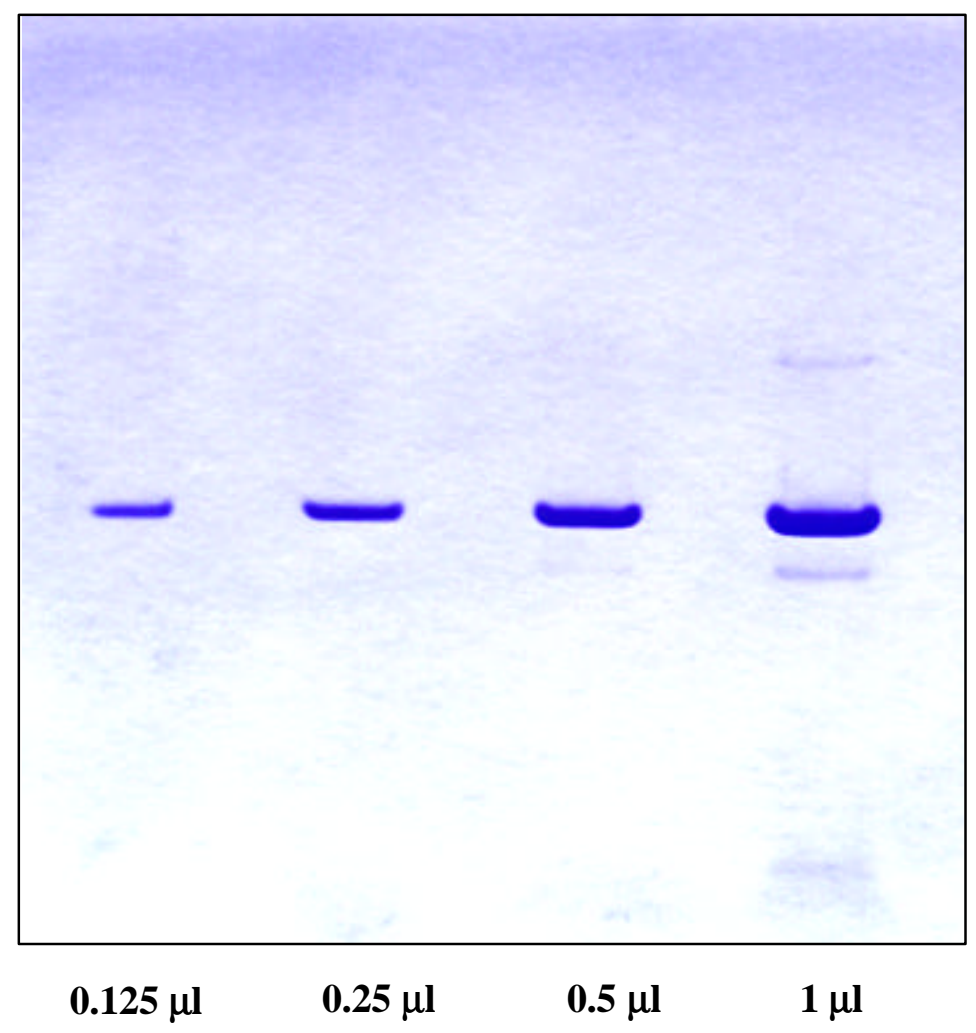

Sample: $\quad 1,863 \mu \mathrm{l}$

$12.5 \mathrm{mg}$

Total activity: $\quad 476,928$ Units 
protein immobilization kit. Using this kit, $20 \mathrm{mg}$ of purified rVCP was covalently immobilized to $2 \mathrm{ml}$ activated sepharose beads for use in chromatography. Using this column, rVCP specific antibodies could be purified from the immunoglobulin samples. After passing $5 \mathrm{ml}$ of purified $\mathrm{IgY}$ through the column, a $\mathrm{NaCl}$ step gradient was used to elute the bound antibodies, see figure 13A. As can be seen, different strengths of antibody affinity are evident. Only the higher $\mathrm{NaCl}$ fractions were then pooled and concentrated. Similar techniques were used in an attempt to purify rabbit anti-rVCP IgG, but were unsuccessful due to very low concentrations of specific antibodies. The low concentration of specific IgG antibodies against VCP found in the immunized rabbit sera was most likely the result of VCP being only mildly immunogenic in mammals. This is due to the great structural similarity between VCP and the mammalian complement regulatory proteins.

To better characterize the affinity-purified anti-rVCP IgY, a Biacore X instrument (University of Western Cape, Cape Town, SA), utilizing surface plasmon resonance technology, was employed. With rVCP covalently immobilized to a CM-5 sensor chip, $10 \mathrm{mM}$ anti-rVCP IgY was injected. As can be seen in figure 13B, strong binding occurs. To test the specificity of this antibody, $10 \mathrm{mM}$ pre-immune (non-affinity purified) $\operatorname{Ig} \mathrm{Y}$ was injected. Figure $13 \mathrm{C}$ shows that no binding was evident, indicating that binding antibodies arose after immunization. Finally, anti-rVCP was pre-incubated with $50 \mathrm{nM} \mathrm{rVCP}$ before injecting. As can be seen in figure 13D, the binding to the chip (with rVCP immobilized) was completely abolished, compared to that seen in figure 13B. The results obtained in these experiments demonstrated the specificity of the anti-rVCP IgY; this antibody was then available for many future immunohistochemistry studies. 
Figure 13. Purification and characterization of anti-rVCP IgY. IgY anti-rVCP was obtained from Washington Biotech through multiple immunization of a single chicken. A) rVCP affinity purification of ant-rVCP IgY using an rVCP column, made by immobilizing rVCP to a Pierce Amino-Link Plus column. Anti-rVCP IgY was eluted using a $\mathrm{NaCl}$ step gradient ranging from $50 \mathrm{mM}$ to $2 \mathrm{M}$. IgY with various affinities can be seen eluting from the column at different $\mathrm{NaCl}$ concentrations. Only the higher affinity antibodies were pooled and concentrated for use in immunohistochemistry and Biocore studies. B) With rVCP immobilized to a CM5 Biacore sensor chip, $10 \mathrm{nM}$ of anti-rVCP IgY was injected and binding was observed. The kinetics of this binding are very strong, as indicated by the extended disassociation time. C) With rVCP immobilized to a CM5 Biacore sensor chip, $10 \mathrm{nM}$ of pre-immune IgY was injected and binding was monitored. No binding was observed, indicating that no anti-rVCP IgY was present. D) With rVCP immobilized to a CM5 Biacore sensor chip, $10 \mathrm{nM}$ of anti-rVCP IgY and $50 \mathrm{nM} \mathrm{rVCP}$ was mixed together, injected, and binding was monitored. Again, no binding was observed, indicating that the antibody was specific to the immobilized protein, rVCP. 

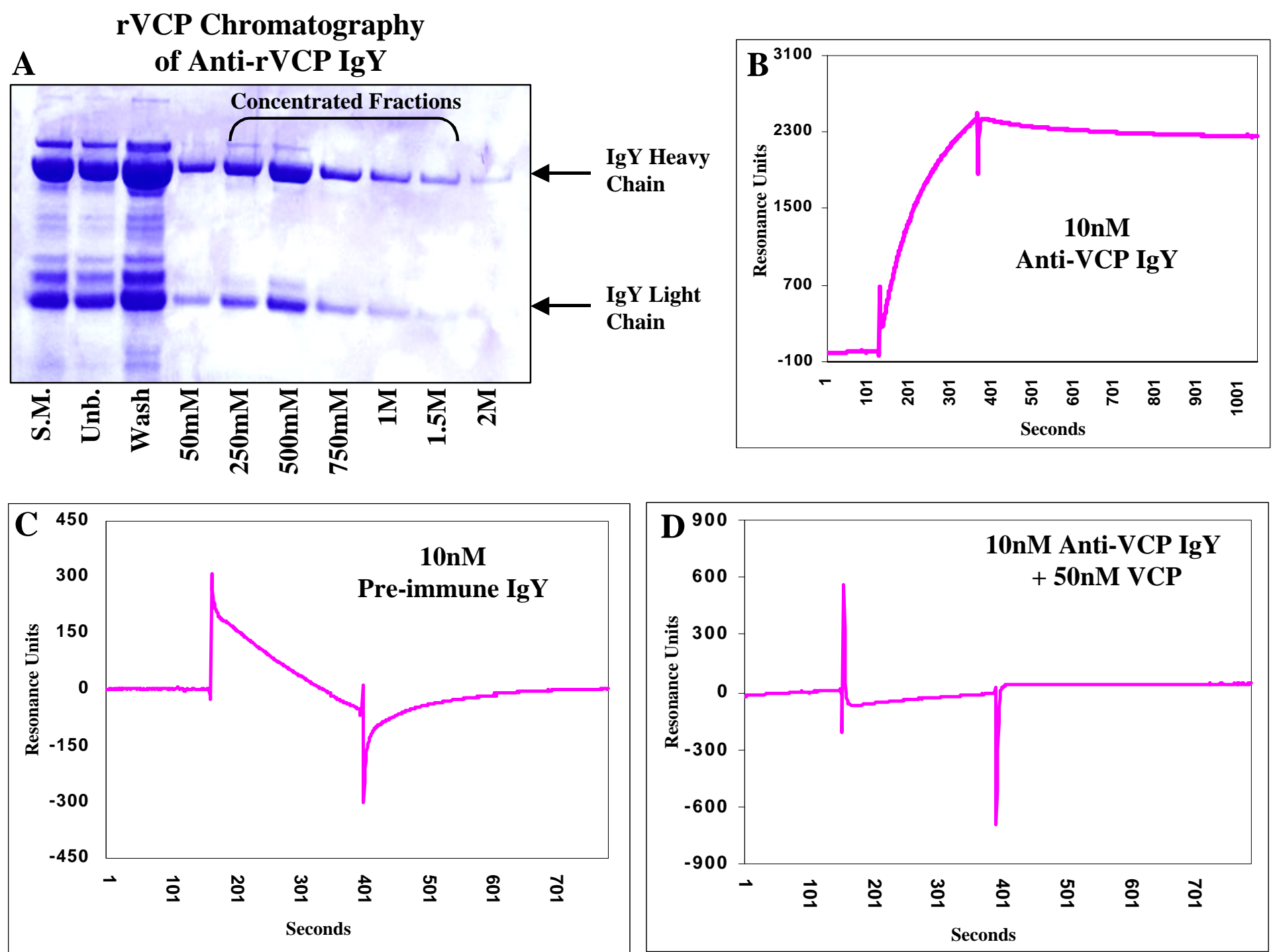


\title{
CHAPTER 3
}

\section{HEPARIN BINDING ACTIVITY OF VCP}

\author{
Introduction to Heparin Binding
}

This chapter contains material adapted from the following publications and manuscript:

Smith S.A., N.P. Mullin, J. Parkinson, S.N. Shchelkunov, A.V. Totmenin, V.N. Loparev, R. Srisatjaluk, D.N. Reynolds, K.L. Keeling, D.E. Justus, P.N. Barlow, and G.J. Kotwal. Conserved surface-exposed K/R-X-K/R motifs and net positive charge on poxvirus complement control proteins serve as putative heparin binding sites and contribute to inhibition of molecular interactions with human endothelial cells: a novel mechanism for evasion of host defense. J. Virol. Jun. 74(12):5659-66, 2000 [Cover of J. Virol. Oct. 74(20), 2000].

Murthy K.H., Smith S.A., Ganesh V.K., Judge K.W., Mullins N., Barlow P.N., Ogata C.M., and Kotwal G.J. Crystal structure of a complement control protein that regulates both pathways of complement activation and binds heparan sulfate proteoglycans. Cell. Jan. 26, 104:301-11, 2001.

Smith S.A., Arjunwadkar S., Krishnasamy G., Judge K., Murthy K.H., Pugh D.R., and Kotwal G.J. Mapping of regions within the vaccinia virus complement control protein involved in binding to key complement components and heparin using surface plasmon resonance. Submitted to Virology, 2002.

The most recently identified property of the multifunctional $\mathrm{VCP}$ is its ability to bind heparin. It has been shown in previous studies, as well as this one, to exhibit lysozyme-like heparin binding activity. Because of this activity, VCP can be taken up by mast cells and possibly persist in the tissue for extended periods of time, helping to preserve the viral habitat (Kotwal et al., 1998b). In vivo studies have indicated that FITC-labeled VCP injected intravenously becomes localized along the endothelium. It has also been shown to reduce chemotactic migration of leukocytes in the presence and 
absence of the chemokine MIP-1 $\alpha$ (Reynolds et al., 2000). This suggests that VCP can bind to heparin-like (heparan sulfate proteoglycans, see figure 14) molecules lining the surface of endothelial cells, blocking chemokine binding and thereby blocking the chemotactic signal.

It is clear that the ability to bind polyanions, such as heparin, is crucial for the function of many immune-regulating proteins. Factor $\mathrm{H}(\mathrm{fH})$, for example, binds to the polyanion sialic acid on the surface of mammalian cells, preventing activation of the alternative pathway (Meri and Pangburn, 1994). Like VCP, fH is made up of SCRs, three of which have been shown to have heparin binding abilities (Blackmore et al., 1998). It is postulated that these heparin-binding domains are responsible for binding sialic acid, thereby preventing alternative pathway activation. The heparin binding regions of many other proteins exhibiting heparin binding activity have been mapped (Cardin and Weintraub, 1989; Cardin et al., 1991; Marino et al., 1999; Sendak and Bensadoun, 1998; Wong et al., 1995; DuBose and Haugland, 1993). VCP, fH, and C4b$\mathrm{BP}$ are the only complement control proteins found to possess this ability (Blom et al., 1999; Blackmore et al., 1998). In most cases (including ours), binding is the result of dense regions of positively charged amino acids. These arginine and/or lysine rich regions vary in sequence and length among heparin binding proteins. Interestingly, the heparin binding sequences used by VCP and its homologues are short and relatively conserved.

We have attempted to further characterize the biological significance of VCP's ability to bind heparin. Using flow cytometry, the amount of specific antibody binding to human endothelial cells - in the presence and absence of VCP - was measured. It was 
Figure 14. Heparan sulfate proteoglycans. Heparan sulfate proteoglycan structure is illustrated, along with their location and the monomers that make up the large polysaccharide chains. Heparan sulfate proteoglycans are present on many cell surfaces. The main body, or peptide core, possesses a short intercellular domain, membrane anchoring region, and extracellular peptide core to which the polysaccharide, glycosaminoglycan, chains are attached. Heparan sulfate proteoglycans are named by the number of sulfate groups per disaccharide unit of the glycosaminoglycan chain; for example, heparan monosulfate contains one sulfate group per disaccharide unit. Also shown are the monomers that can make up the glycosaminoglycan chain. Depending on the degree of sulfation, heparan sulfate proteoglycans may have varying degrees of electronegative charge. 


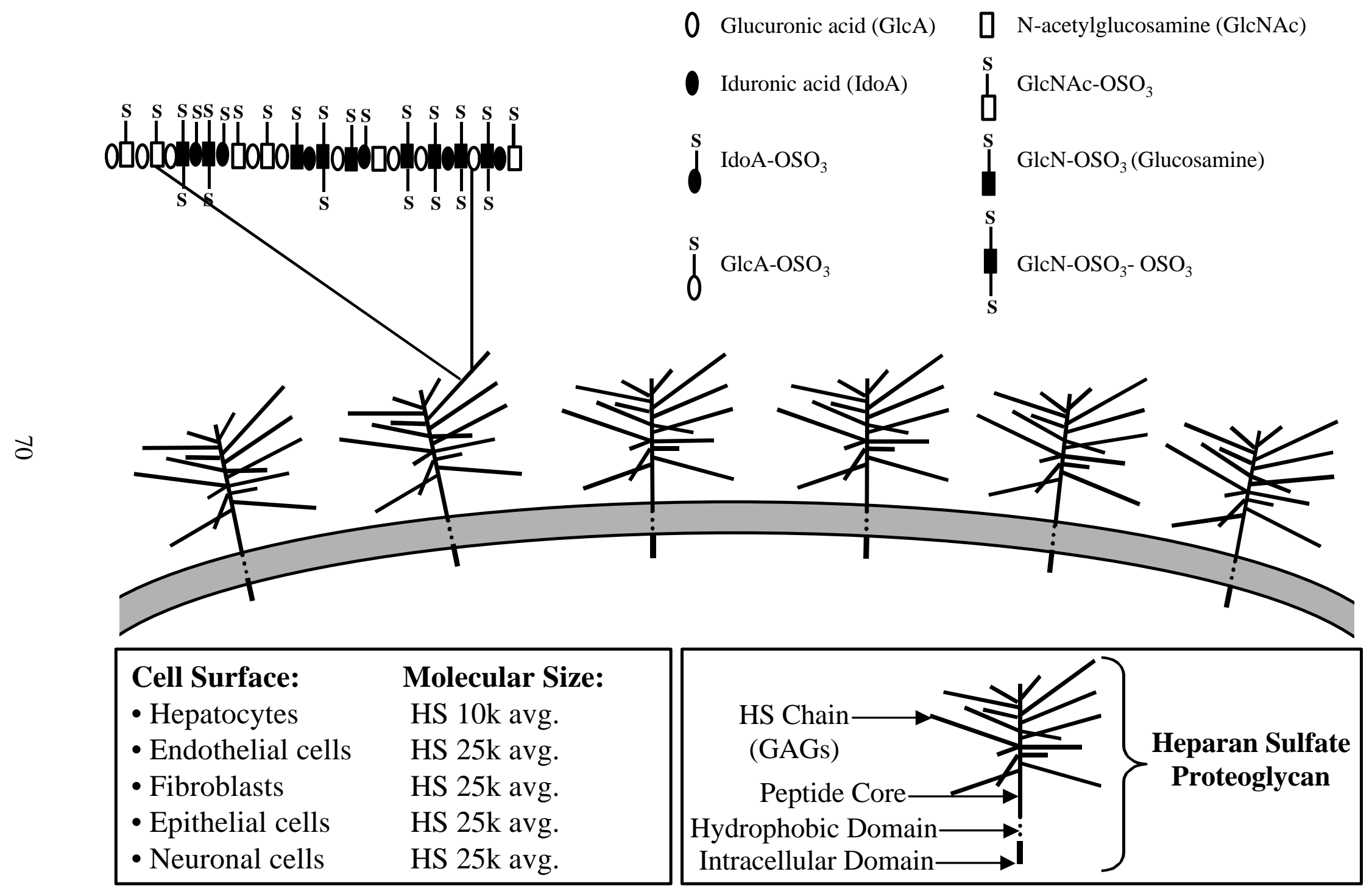


found that $\mathrm{VCP}$ was able to inhibit antibody binding to MHC class I molecules on human endothelial cells. This suggests that VCP can interfere with molecular interactions with infected cells and could prevent antibody-dependent cell-mediated cytotoxicity (ADCC) as well as other cytotoxic cell interactions with target cells. The ability of VCP to bind heparin-like molecules suggests that it plays many roles and therefore may have a variety of applications. It is for these reasons that we have been interested in obtaining a better understanding of the molecular basis for the VCP-heparin interaction. Through examination of several recombinant VCP fragments, it has now been determined that the percentage of positively charged amino acids, overall charge, and the number of putative heparin binding sites, are all important factors governing the heparin binding ability of rVCP. In addition, the heparin binding ability of rVCP and the various truncated rVCP molecules was tested using surface plasmon resonance (SPR) binding analysis (Biacore $\mathrm{X}^{\circledR}$ ) with heparin immobilized to the sensor chip. Positive binding was determined by subtraction of ligand channel readings from the readings of the control channel, and each experiment was repeated several times to confirm results. Next, in order to test whether the putative heparin binding site, $\mathrm{K} / \mathrm{R}-\mathrm{X}-\mathrm{K} / \mathrm{R}$, was actually involved in this interaction, synthetic peptides (tri-peptides) were designed to resemble the putative heparin binding sites present on rVCP, and competition assays were performed. The presence of this synthetic heparin binding site resulted in a stronger binding between rVCP and heparin, which was believed to result from the bridging of the protein's central net negative charge. With the results of these assays in hand, attempts were then made to purify rVCP 2,3 using this technique. Finally, due to the extreme cost of heparin chromatography, dextran sulfate chromatography was also investigated. As a result of 
the similar high degree of sulfation, rVCP was found to bind very effectively to sulfated dextran beads.

Utilizing the data collected in the above experiments, an NMR model of rVCP was created and the positions of the heparin binding sites highlighted. Two areas, located at each end of the protein, were identified as being involved in binding. In addition, with the X-ray crystal structure in hand, an accurate model of the electrostatic field distribution across rVCP could be predicted and illustrated. Finally, spatial homology with other well-characterized heparin binding domains was investigated for comparison. 


\section{$\underline{\text { Materials and Methods }}$}

\section{Flow Microfluorimetric Analysis:}

Ratchapin Srisatjaluk and David Reynolds, Department of Microbiology and Immunology, aided in all experiments involving flow microfluorimetric analysis. Human umbilical cord vascular endothelial cells (HUVECs) were obtained from the American Type Culture Collection (ATCC, Rockville, MD) at passage thirteen. Monolayer cultures were maintained using F12K Ham's media supplemented with $10 \%$ fetal bovine serum, $30 \mu \mathrm{g} / \mathrm{ml}$ endothelial cell growth supplement (Sigma), and $100 \mu \mathrm{g} / \mathrm{ml}$ heparin (Sigma), at $37^{\circ} \mathrm{C}$ in humidified air containing $5 \% \mathrm{CO}_{2}$. Cells were cultured to approximately $80 \%$ confluency in $75 \mathrm{~cm}^{2}$ vented flasks coated with $1.5 \%$ gelatin in phosphate buffered saline. Cells were trypsinized ( $0.25 \%$ trypsin, $1 \mathrm{mM}$ EDTA, Sigma), and $4 \times 10^{5}$ cells $/ \mathrm{ml}$ were placed in a 6-well flat-bottom culture plates $(3.5 \mathrm{~cm}$ diameter, Falcon, Lincoln Park, $\mathrm{NJ})$, coated with $1.5 \%$ gelatin, and incubated for $24 \mathrm{~h}$ in F12K media without growth factor (Lian et al., 1996). In order to determine the ability of $\mathrm{rVCP}, \mathrm{rVCP} \sim 3,4$, and $\mathrm{HBP}$ to bind HUVECs, duplicate wells ( $2 \mathrm{ml}$ each) were trypsinized and rinsed with FTA hemagglutination buffer (Becton Dickinson) and stained for 30 min on ice with either 20 $\mu \mathrm{g}$ of FITC-labeled rVCP, $30 \mu \mathrm{g}$ of FITC-labeled rVCP 3,4, or $30 \mu \mathrm{g}$ of FITC-labeled HBP. For analysis of antibody interaction with cell surface class I HLA-ABC molecules, triplicate wells ( $2 \mathrm{ml}$ each) were trypsinized and rinsed with FTA hemagglutination buffer and stained for $30 \mathrm{~min}$ on ice with $0.25 \mu \mathrm{g}$ of phycoerythrin-conjugated mouse antihuman HLA-ABC mAb (Caltag, Burlingame, CA), or a mouse IgG2a mAb (an isotype-match negative control) in the presence or absence of 2 or $5 \mu \mathrm{g}$ of VCP $-90-98 \%$ pure from natural infection. After incubation, cells were rinsed three times in FTA 
buffer, and then fixed in Hank's balanced salt solution (HBSS) containing 2\%

paraformaldehyde. Before staining, cell cultures were assessed for viability by trypan blue dye exclusion, and the cells were found to be $>95 \%$ viable. The percentage of positively stained cells was determined using a flow cytometer (Becton Dickinson FACScan, San Jose, CA) equipped with a single 15-mW argon laser tuned to $488 \mathrm{~nm}$, technical assistance and use of the FACS facilities was provided by Dr. Sam Welhausen of the J. Graham Brown Cancer Center. Forward and $90^{\circ}$ angle light scatter, integrated log phycoerythrin and FITC fluorescence signals were collected and analyzed.

Variability between duplicate samples was less than $10 \%$. To compensate for any background fluorescence, the control threshold was set less than $1 \%$ binding of control mAbs. Data were acquired from analysis of $>3000$ events. A single homogenous cell population was indicated as detected by forward and $90^{\circ}$ light scatter.

\section{Heparin Binding Ability:}

In order to establish a basis for comparing heparin binding ability, $10 \mu \mathrm{g}$ each of bovine serum albumin (BSA), heparin binding protein (HBP)[described earlier (Flodgaard et al., 1991)], lysozyme, and MIP-1 $\alpha$ were pooled, dissolved in $1 \mathrm{ml}$ of ultapure water, and passed through a $1 \mathrm{ml}$ HiTRAP heparin column and the unbound material collected. The bound proteins were rinsed with $1 \mathrm{ml}$ of ultrapure water and then eluted with increasing sodium chloride concentrations ranging from $250 \mathrm{mM}$ up to $4.0 \mathrm{M}$. Next, $20 \mu \mathrm{g}$ of purified $\mathrm{rVCP} \sim 1,2, \mathrm{rVCP} 2,3, \mathrm{rVCP} \sim 3,4, \mathrm{rVCP}$ or $10 \mu \mathrm{g}$ of wild type VCP (from VV infection of cell culture) were each dissolved in $1 \mathrm{ml}$ of ultrapure water and passed through separate $1 \mathrm{ml}$ HiTRAP heparin columns and the unbound materials 
collected. After washing the column with $1 \mathrm{ml}$ of ultrapure water, the proteins were eluted with sodium chloride concentrations ranging from $250 \mathrm{mM}$ to $2.5 \mathrm{M}$. The fractions were then separated using SDS-PAGE and silver stained. Densitometric readings were taken using the AlphaImager 2000 software system. Finally, $0.5 \mathrm{ml}$ of the fraction containing the protein was concentrated by ultrafiltration and its activity tested using the hemolysis assay.

Sequencing of the VCP Homologue in MPV:

Sequencing was done by Alexei Totmenin, from the Department of Molecular Biology of Genomes, State Research Center of Virology and Biotechnology Koltsovo, Novosibirsk Region, Russia, as described previously (Shchelkunov et al., 1998).

\section{Biacore Instrumentation:}

Instruction and use of the Biacore $\mathrm{X}^{\circledR}$ facilities was provided by David Pugh and Jasper Rees from the Department of Biochemistry, University of Western Cape, Cape Town, South Africa. Carboxylated dextran sensor chip CM5 was chosen for amine coupling. Chip was docked and primed in a Biacore $\mathrm{X}^{\circledR}$ instrument. Hepes-buffered saline (HBS) (Biacore, Biagrade) was used as the running buffer throughout the study. Standard amine coupling was used to immobilize heparin to the sensor chip - see figure 15 for amine coupling procedure. First, channel one was selected with a flow rate of 10 $\mu 1 /$ min. The surface of the sensor chip was then activated by injecting $35 \mu l$ of a freshly prepared 1:1 mixture of N-hydroxysuccinimide (NHS) to N-ethyl-N'(dimethylaminopropyl)-carbodiimine (EDC). Directly following surface activation, $30 \mu 1$ 
Figure 15. Standard amine coupling procedure for Biacore instrument. The carboxylated dextran sensor chip (CM5) was chosen for amine coupling. Chip was docked and primed in a Biacore $\mathrm{X}^{\circledR}$ instrument. The surface of the sensor chip was then activated by injecting a freshly prepared 1:1 mixture of N-hydroxysuccinimide (NHS) to N-ethyl-N'-(dimethylaminopropyl)-carbodiimine (EDC). Directly following surface activation, a mixture of streptavidin (the ligand) $(1 \mathrm{mg} / \mathrm{ml})$ and coupling buffer $3(0.1 \mathrm{M}$ glycine $\mathrm{pH} 4.8$ ) was injected. The surface was then deactivated (unreacted groups were blocked) by injecting $20 \mu \mathrm{l}$ ethanolamine $\mathrm{HCl}(1 \mathrm{M}, \mathrm{pH} 8.5)$. 


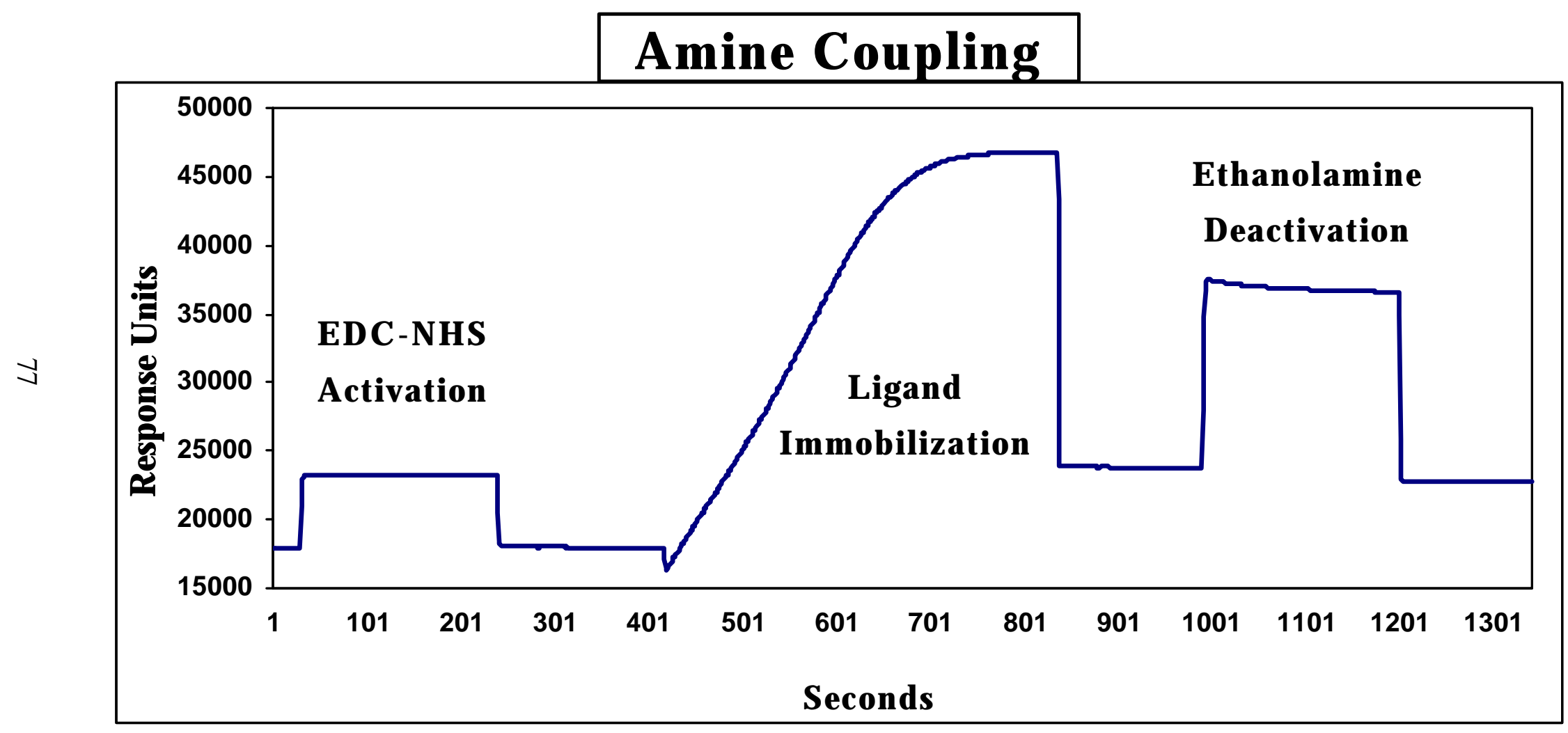


of a mixture of $5 \mu \mathrm{l}$ streptavidin $(1 \mathrm{mg} / \mathrm{ml})$ and $95 \mu$ coupling buffer $3(0.1 \mathrm{M}$ glycine $\mathrm{pH}$ 4.8) was injected. The surface was then deactivated (unreacted groups were blocked) by injecting $20 \mu \mathrm{l}$ ethanolamine $\mathrm{HCl}(1 \mathrm{M}, \mathrm{pH} 8.5)$. Surface regeneration was frequently achieved by injecting $10 \mu \mathrm{l}$ of Biacore regeneration solutions 1,2 , or 3 . In order to arrive at satisfactory regeneration in studies testing heparin binding of rVCP, 1-10 $\mu 1$ injections of 50-70 $\mathrm{mM} \mathrm{NaOH}$ were used. If regeneration was not adequate after the first injection, more than one injection was tried. Care was taken not to damage ligand on chip surface. BiaEvaluation 3.0 software was used to evaluate all data collected. For comparison, data is presented by displaying both ligand channel and control channel for all binding studies.

In order to test the interaction between heparin and rVCP, heparin was immobilized to a CM5 sensor chip in the following manner. Using standard amine coupling, streptavidin (Sigma) was immobilized to channel one of the sensor chip. Next, heparin-albumin-biotin (Sigma) was passed over the streptavidin, resulting in binding of this heparin coated albumin molecule (albumin with heparin and biotin covalently bound) to the sensor chip. Finally, the interaction between rVCP and heparin could be measured. The interaction of various fragments of rVCP with heparin was also tested.

\section{Tri-peptide Studies:}

To test the hypothesis that synthetic peptides resembling the putative heparin binding sites could interfere with binding of rVCP to heparin by competitive inhibition, three tri-peptides were purchased from Bio-Synthesis Incorporated. The KRR tri-peptide was synthesized to act as the competitive inhibitor and both negatively charged (DID) and neutral (GSS) tri-peptides were used as a control. In the presence and absence of 
various concentrations ( $300 \mu \mathrm{g}-3 \mathrm{mg}$ ) of each tri-peptide, heparin chromatography of rVCP was performed. The tri-peptide was suspended in water in the presence of $300 \mu \mathrm{g}$ rVCP. This starting material was then passed through a $1 \mathrm{ml}$ HiTRAP heparin column and the unbinding material was collected. The column was then washed and the protein was eluted with a $\mathrm{NaCl}$ step gradient ranging from $100 \mathrm{mM}$ to $500 \mathrm{mM}$. The fractions were then visualized using SDS-PAGE and coomassie blue staining. The rVCP containing fractions were then compared between tri-peptides in order to determine the effect that they had on the heparin binding ability of rVCP.

\section{Model of rVCP:}

The structure of the two unknown VCP modules (VCP SCR 1 and VCP SCR 2) were modeled by homology with the four known VCP module structures: VCP SCR 3, VCP SCR 4, fH SCR 15 and fH SCR 16, using the program MODELLER (Sali and Blundell, 1993). Five models of each module were created. The model which was closest to the average structure was used in the construction of the rVCP 1-4 model. Construction of the rVCP 1-4 was undertaken using the molecular visualization package, InsightII (Msi Inc. San Diego, Ca). This was achieved by simply bonding the two modeled VCP structures to the NMR derived structure of the $\mathrm{rVCP} \sim 3,4$ module pair (Wiles et al., 1997). No attempt was made to accurately model the junctions between modules 1 and 2 and modules 2 and 3. Rather, torsion angles in the two residues linking the module pairs were set to give a relatively extended conformation. 


\section{X-Ray Crystallography:}

Dr. Krishna Murthy from the Center for Biophysical Science and Engineering, University of Alabama at Birmingham performed the X-ray crystallography of rVCP. Two crystal forms, form I and form II, of rVCP expressed in yeast were grown. Both were obtained using a protein concentration of $5-12 \mathrm{mg} / \mathrm{ml}$ in $20 \mathrm{mM}$ TRIS, $\mathrm{pH} 7.5$ (form I) and 8.3 (form II), $100 \mathrm{mM} \mathrm{NaCl}$. Form I crystals were grown using 10\% PEG 6000 and form II crystals using 8-12\% PEG 8000 in hanging drop vapor diffusion experiments over $7-10$ days at $20^{\circ} \mathrm{C}$. 


\section{$\underline{\text { Results }}$}

Specific Antibody Blocking to HUVECs:

In order to determine the biological consequences of heparin binding by $\mathrm{VCP}$, we tested whether VCP was able to block functional molecular interactions with human umbilical cord vascular endothelial cells (HUVECs). First, the ability of FITC-labeled rVCP to bind heparin was tested by chromatography. As can be seen in figure 16 , the heparin binding ability of rVCP is diminished due to labeling. However, approximately $\sim 25 \%$ of the labeled material retains functional heparin binding ability. The ability of rVCP, $\mathrm{rVCP} 3,4$, and HBP to bind the surface of HUVECs was then tested using flow cytometric analysis. As shown in figure 17A and B, all three proteins possess the ability to bind strongly to HUVECs. A 3.0, 2.9, and 5.4 fold increase in mean fluorescent intensity (MFI) over the control was observed when $20 \mu \mathrm{g}$ FITC-labeled rVCP, $30 \mu \mathrm{g}$ FITC labeled rVCP 3,4, or $30 \mu \mathrm{g}$ FITC labeled HBP (respectively) was added to the suspension of HUVECs. Next, using flow cytometric analysis of HUVECs treated with mouse monoclonal antibodies to human MHC class I molecules - in the presence and absence of VCP - VCP was shown to be capable of modulating antibody binding in a dose-dependent manner. As shown in figure 17C, addition of $5 \mu \mathrm{l}(2 \mu \mathrm{g})$ of VCP to antibody treated HUVECs, reduced antibody binding from $91.5 \%$ down to $75.4 \%$. An additional $15 \mu \mathrm{l}$ (5 $\mu \mathrm{g}$ total) of VCP reduced antibody binding from $91.5 \%$ to $61.0 \%$ suggesting that it is blocking in a dose-dependent manner. 
Figure 16. Heparin binding of FITC-labeled rVCP. Heparin purification of rVCP labeled with FITC. Most of the FITC rVCP sample passed through the HiTRAP heparin column with out binding. Only $\sim 25 \%$ of the sample bound heparin with the same affinity as the unlabeled protein, see figure 7 for comparison. This observation may be due to FITC masking of the heparin binding sites on rVCP. 
Heparin Binding of FITC Labeled rVCP

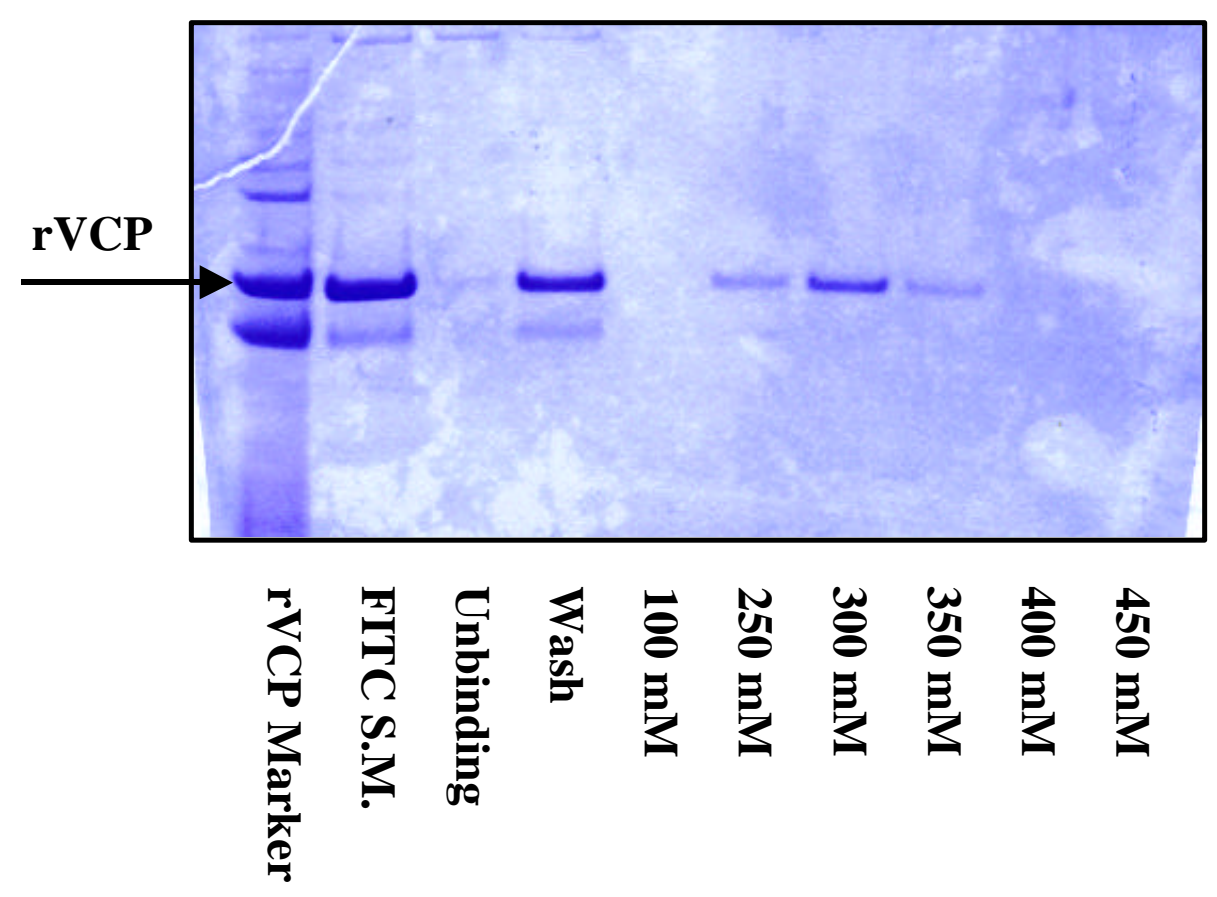


Figure 17. Representative histograms showing that VCP is able to reduce mouse antihuman HLA class I antibody binding to HUVECs. The results of flow microflourimetric analysis are as follows: A1) No treatment control A2) Binding of FITC-labeled rVCP to HUVECs B1) No treatment control B2) Binding of FITC-labeled rVCP SCR $(3,4)$ to HUVECs B3) Binding of FITC-labeled HBP to HUVECs C1) Nonspecific mouse monoclonal antibody (isotype-matched mouse IgG2a mAb) binding to HUVECs cells (negative control) C2) Mouse monoclonal antihuman HLA-ABC antibody binding to HUVECs (positive control) C3) Mouse monoclonal antihuman HLAABC antibody binding to HUVECs in the presence of $5 \mu \mathrm{l}(2 \mu \mathrm{g})$ of VCP C4) Mouse antihuman HLA-ABC antibody to HUVECs in the presence of $20 \mu \mathrm{l}(5 \mu \mathrm{g})$ of VCP. 

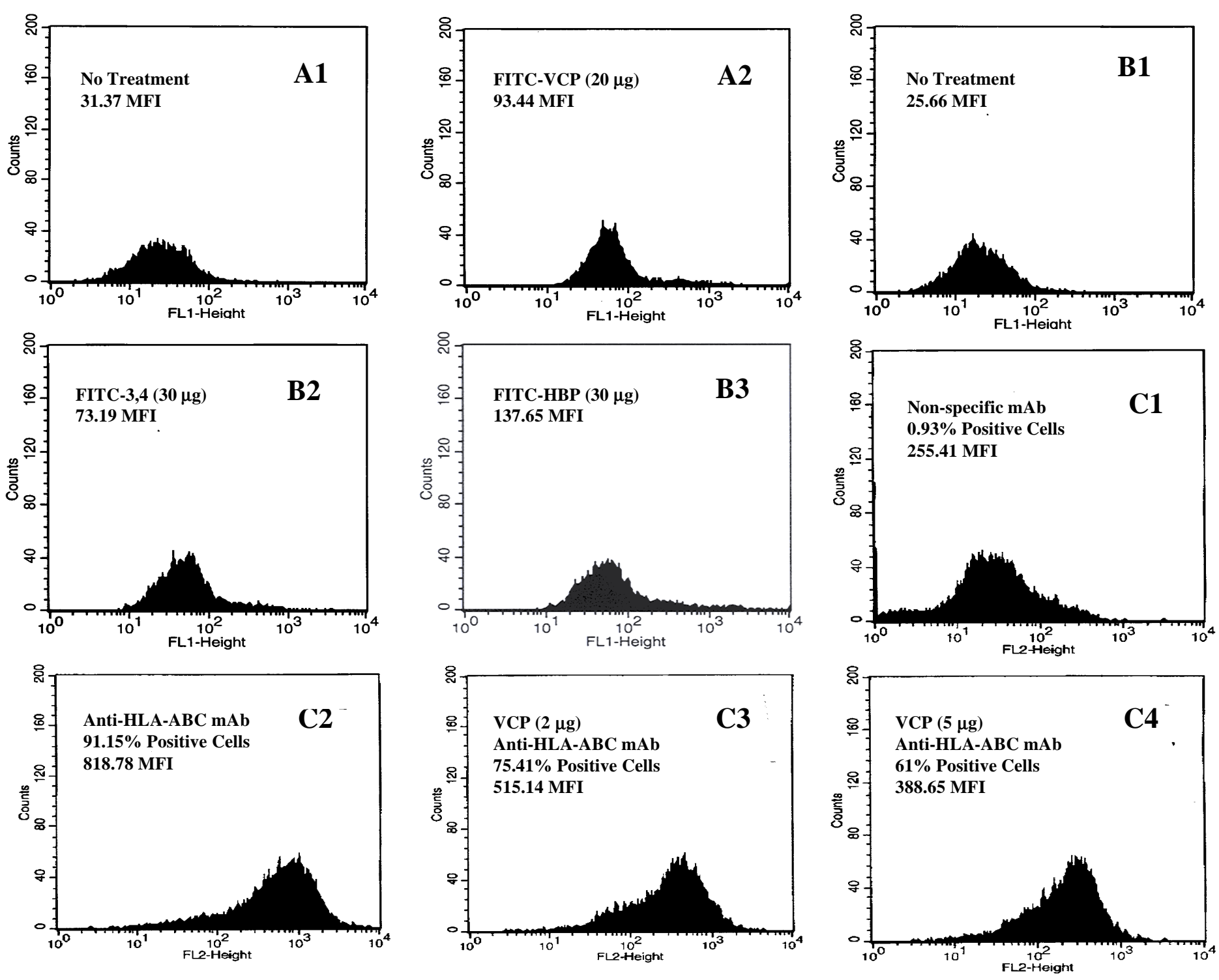


\section{Heparin Binding of rVCP and rVCP Fragments:}

To calibrate the HiTRAP heparin column, several proteins having different binding affinities for heparin were passed through the column and eluted with increasing sodium chloride concentrations. SDS-PAGE analysis of the eluted fractions containing these proteins of various heparin-binding activities can be seen in figure 18. Analysis suggests that bovine serum albumin (BSA) does not bind heparin, since it is present only in the unbound and wash fractions. Lysozyme and MIP-1 $\alpha$, which bound heparin with moderate and equal affinity, were contained within the $500 \mathrm{mM}$ and $750 \mathrm{mM}$ sodium chloride fractions. HBP, showing the highest affinity for heparin, was eluted by a sodium chloride concentration of $2.0 \mathrm{M}$.

In order to better characterize the heparin binding ability of the VCP molecule, VCP, recombinant VCP, and various recombinant segments of VCP were passed through HiTRAP heparin columns and analyzed using SDS-PAGE, shown in figure 19. The results suggest that the full-length natural and recombinant proteins bind heparin with close to or equal affinity as lysozyme and MIP-1 $\alpha$; both VCP and rVCP were concentrated primarily in the $500 \mathrm{mM}$ and $750 \mathrm{mM}$ sodium chloride fractions. PAGE analysis of the various recombinant VCP segments revealed data that are even more interesting. Recombinant $\mathrm{rVCP} \sim 1,2$ and $\mathrm{rVCP} \sim 3,4$ bound heparin with the same strength as the full-length protein, eluting once again at $500 \mathrm{mM}$ and $750 \mathrm{mM}$. In contrast, recombinant $\mathrm{rVCP} 2,3$ did not bind heparin at all and was found primarily in the unbound and wash fractions. The activity of the purified proteins was then tested using the hemolysis assay. The results indicate that only the full-length protein inhibits lysis of sensitized sheep red blood cells - anywhere from 60 to $90 \%$ inhibition. The rVCP 
Figure 18. PAGE analysis of the heparin binding activity of BSA, HBP, lysozyme, and MIP-1 alpha. In order to calibrate the HiTRAP heparin column, various proteins (BSA, HBP, lysozyme, and MIP-1 $\alpha$ ) with differing affinities for heparin were passed through the column and eluted with sodium chloride concentrations ranging from 250 $\mathrm{mM}$ to $4.0 \mathrm{M} . \mathrm{M}=$ molecular weight marker, S.M. = starting material, Unb. = unbound fraction, $\mathrm{W}=$ wash . 


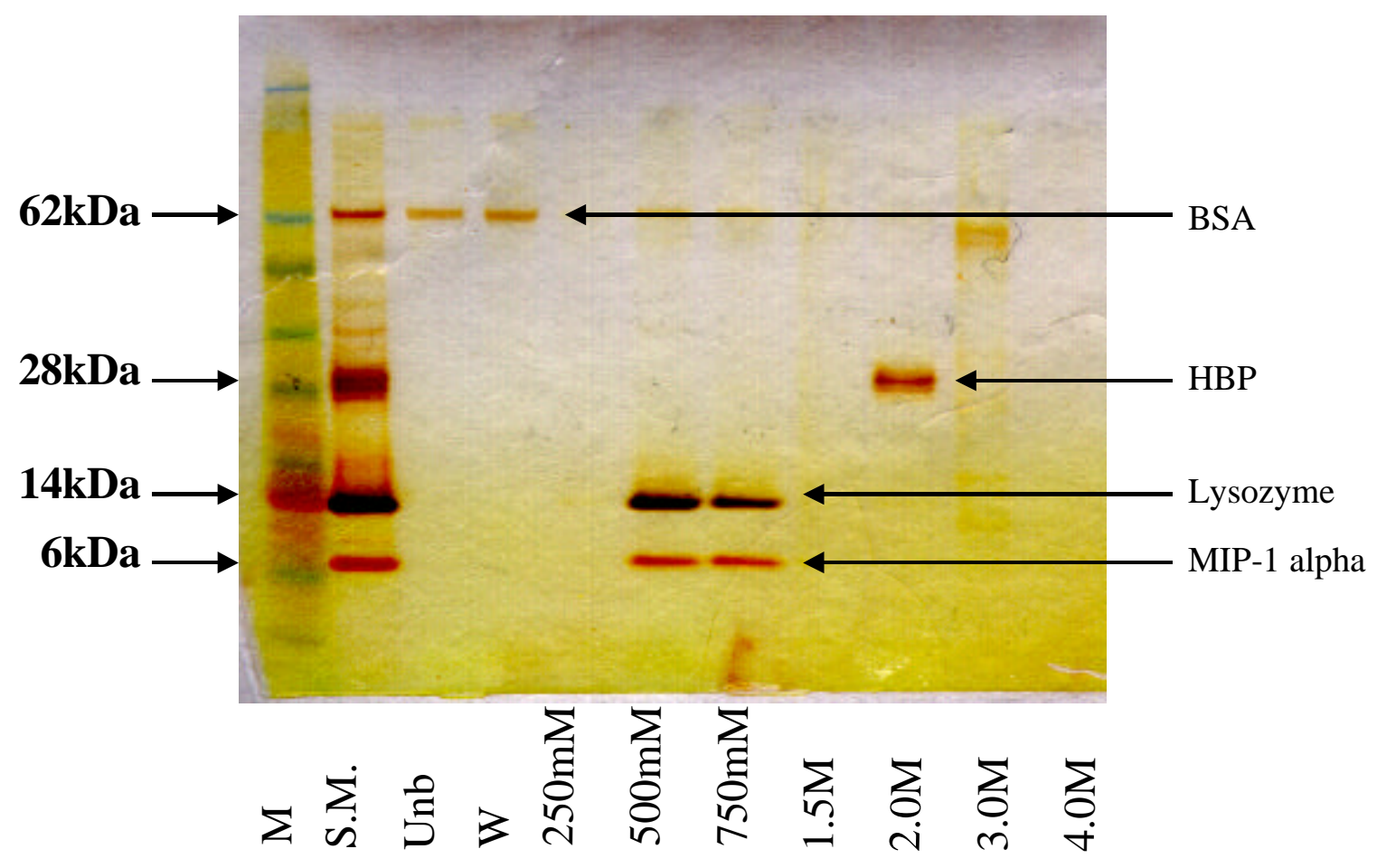


Figure 19. PAGE analysis of the heparin binding activity of VCP and rVCPs. VCP and various rVCPs were passed through separate HiTRAP heparin columns and eluted with sodium chloride concentrations ranging from $250 \mathrm{mM}$ to $2.5 \mathrm{M}$. The fractions were collected, run on SDS PAGE, silver stained, and the band densities measured. The results are shown as follows: A) recombinant $\mathrm{rVCP}$ SCR $(1,2)$ B) recombinant rVCP SCR $(2,3)$ C) recombinant rVCP SCR $(3,4)$ D) recombinant rVCP E) VCP from the natural infection process. For gels A-E, $\mathrm{M}=$ molecular weight marker, S.M. = starting material, Unb. $=$ unbound fraction, $\mathrm{W}=$ wash, $\mathrm{VCP}=\mathrm{VCP}$ from natural infection. $\mathrm{F}$ ) Densitometric scanning of above gels A-E. 


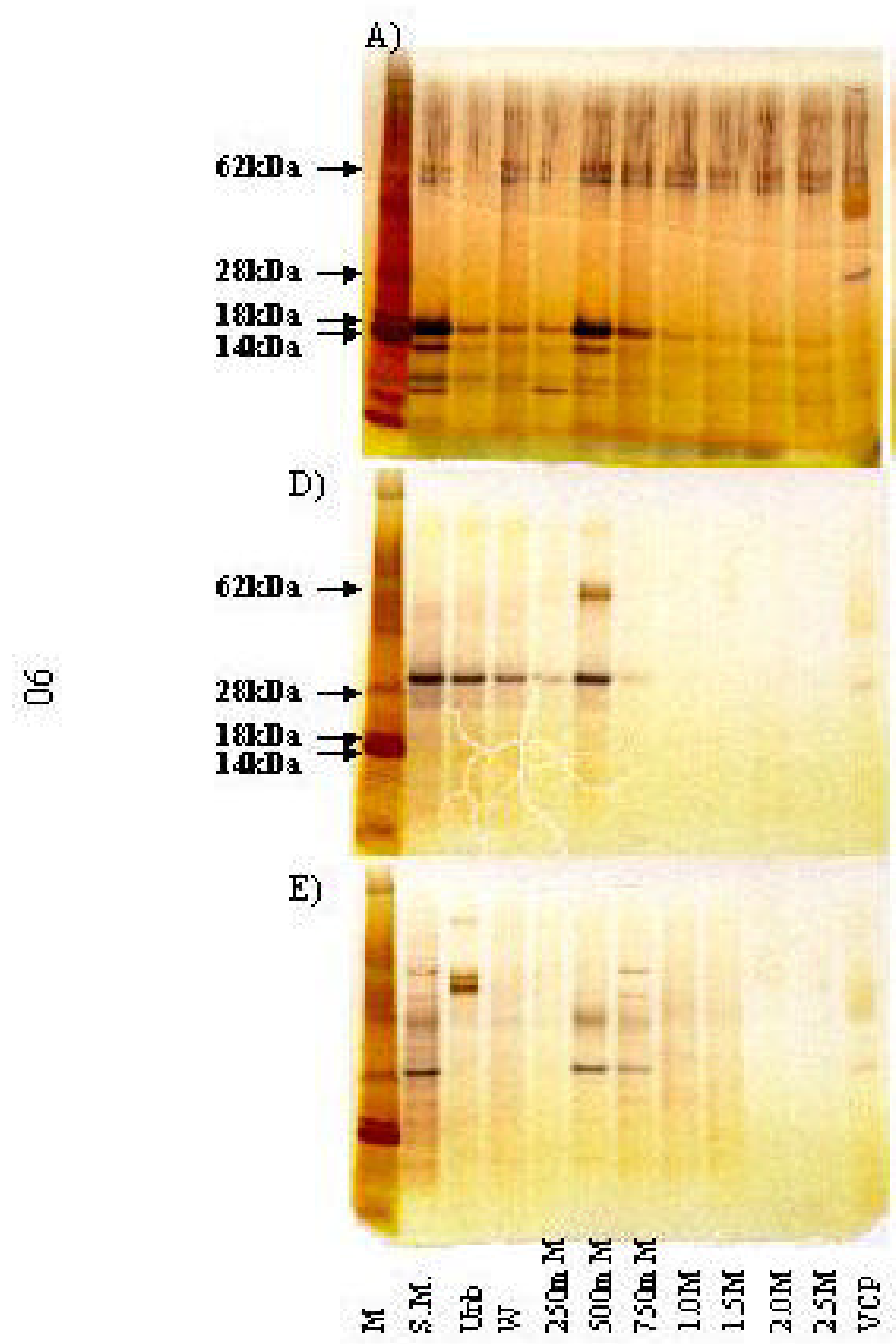

B)

C)
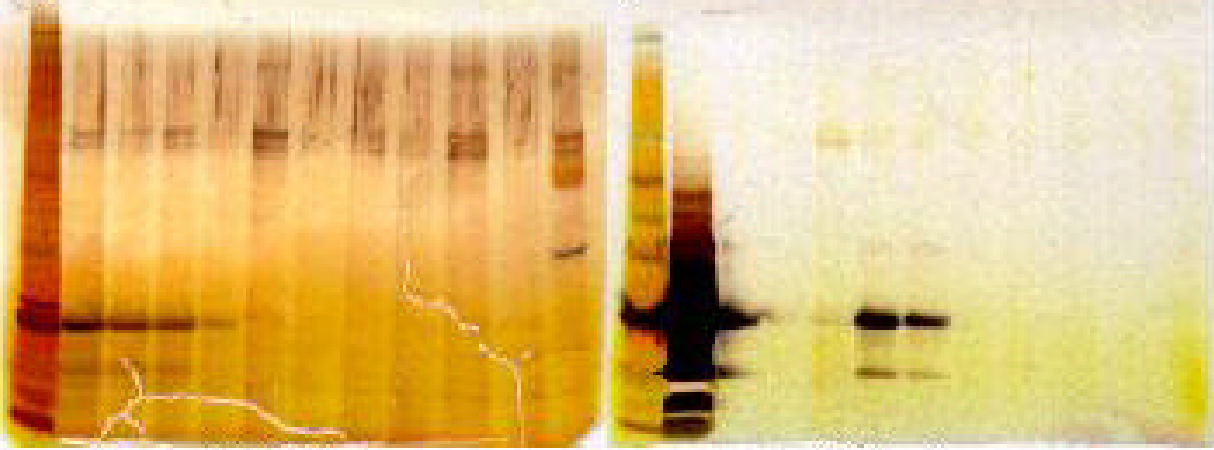

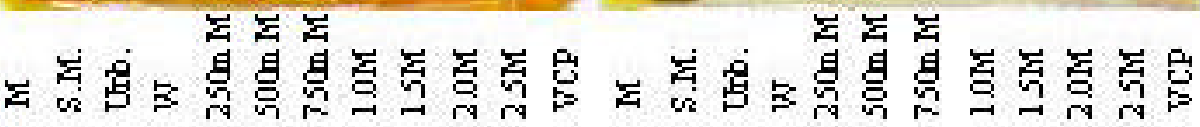
F)

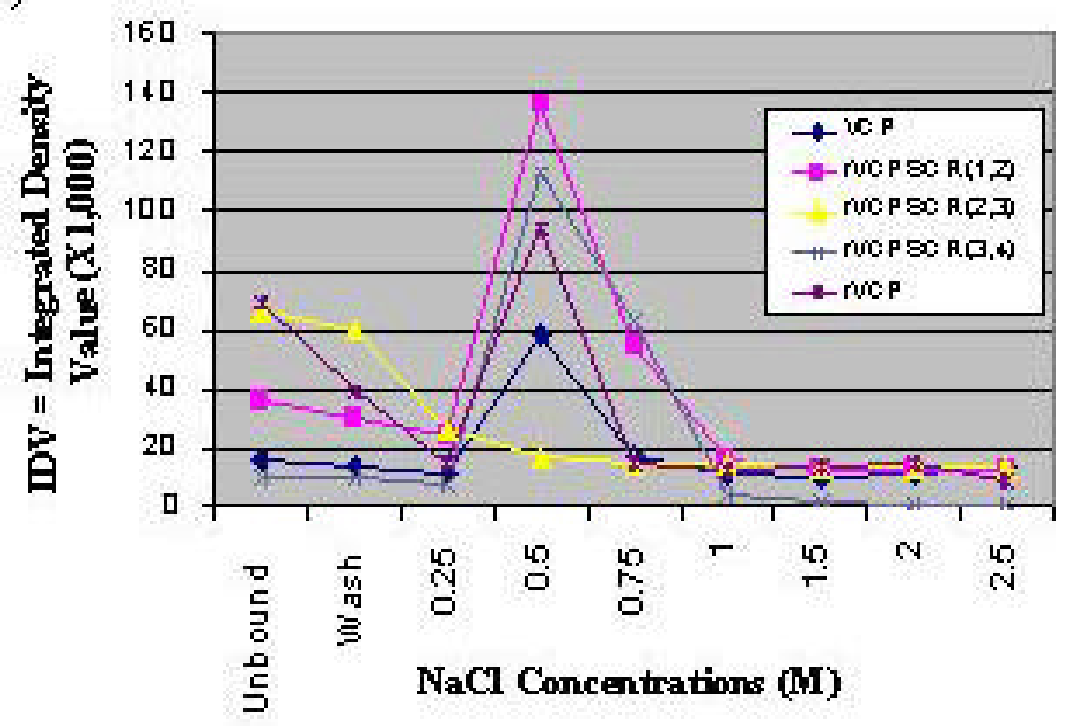


segments showed no inhibition of lysis, suggesting that the whole protein is needed to block complement activation. The naturally truncated VCP homologue produced by monkeypox virus (MPV)(shown in figures 20 and 21), which lacks almost the entire fourth SCR, has been shown to inhibit hemolysis of sensitized sheep red blood cells. Thus, this indicates that it is blocking the classical pathway of complement activation.

\section{Conserved $K / R-X-K / R$ Sites:}

To more precisely identify the molecular basis behind the VCP-heparin interaction, the amino acid sequences were analyzed using the MacVector software system. Putative heparin binding sites $(\mathrm{K} / \mathrm{R}-\mathrm{X}-\mathrm{K} / \mathrm{R})$ were first identified - shown in figure 20 along with the sequence alignment of different poxvirus VCP homologues and predicted to be located on the surface of the protein. The amino acid sequences were then scanned to determine the total number of positive amino acids $(K+R)$, the percentage made up of these positively charged amino acids $(\% \mathrm{~K}+\mathrm{R})$, and the overall $\mathrm{pI}$ of the protein - the results are summarized in figure 21. All of the proteins shown to bind heparin had an overall pI of greater than 7.0, and more importantly, were made up of greater than $9 \%$ positively charged amino acids. The results also suggest that SCR 1 and SCR 4 contribute the most to this interaction. Although the results show there is no simple answer to how VCP binds heparin, it is clear that the overall positive charge, number of putative binding sites, and the percentage of positive amino acids making up the protein, have a significant effect on its ability to interact with and bind heparin. 
Figure 20. Sequence alignment including termini of rVCP constructs and putative heparin binding sites. Multiple alignment of the four short consensus repeats (SCR) from orthopoxviruses VAC-COP (vaccinia virus, Copenhagen strain), VAC-WR (vaccinia virus, western reserve strain), CPV-GRI (cowpox virus, Russian isolate from human patient), CPV- BRI (cowpox virus, Brighton strain), VAR-BSH (variola virus, Bangladesh strain), VAR-IND (variola major virus, Indian strain), VAR-GAR (variola minor virus, alastrim Garcia strain), and MPV-ZAI (monkeypox virus, isolated from a human patient from Zaire in 1996). The putative heparin binding sites (K/R-X-K/R) are marked with solid bars, arrows indicate the termini of the rVCP constructs, and the cysteines are highlighted. See figure 57 for disulphide bonding pattern. 


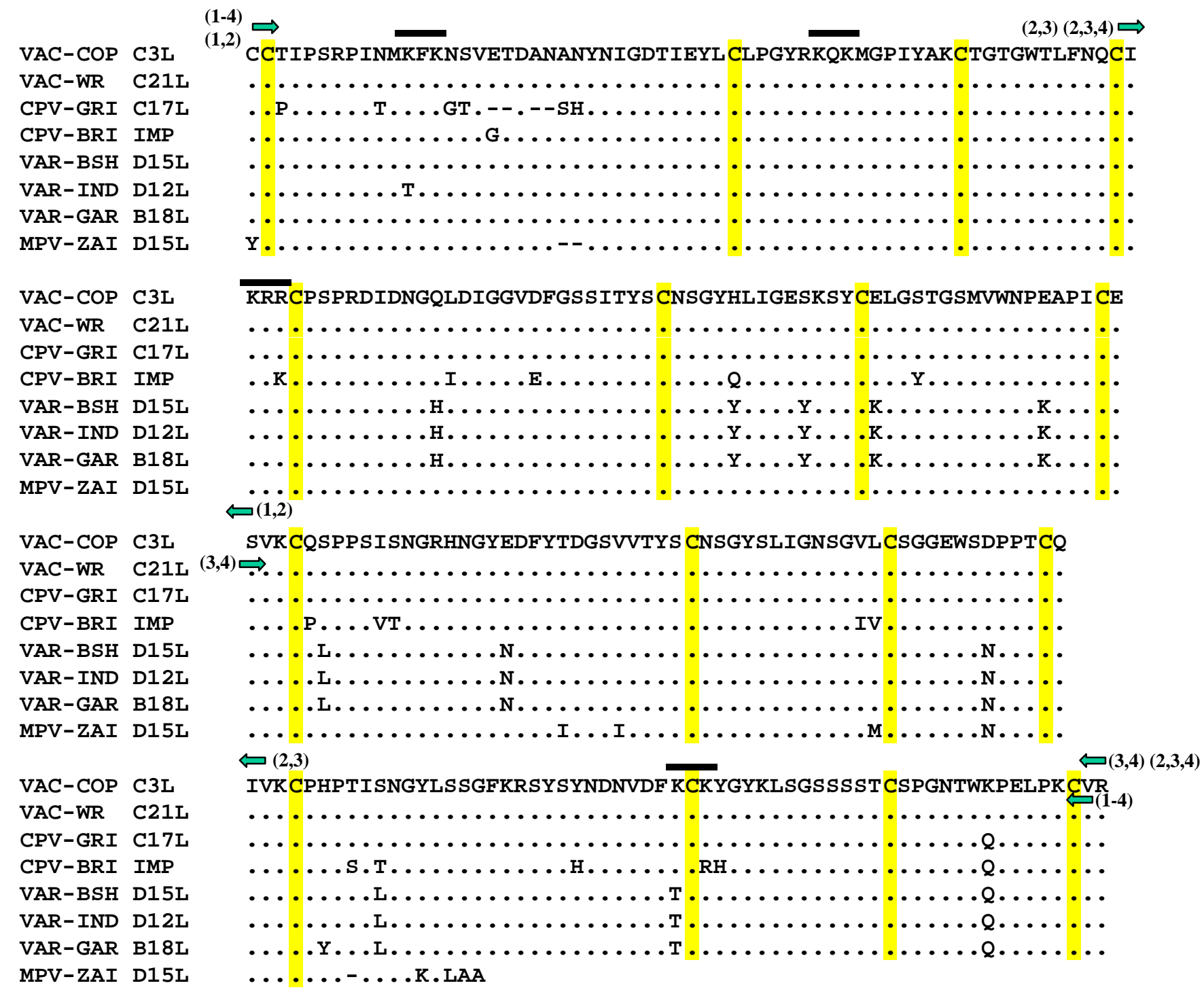


Figure 21. Structure-function summary table of VCP, VCP homologues, and rVCPs. VCP/IMP/SPICE, MPV homologue of VCP, recombinant VCP, and four overlapping recombinant segments of VCP with 2 or 3 SCRs are shown along with their ability to inhibit hemolysis of sensitized sheep red blood cells and/or bind heparin. Also listed are the number of positively charged amino acids $(K+R)$ found in the protein, percentage of positively charged amino acids $(\% \mathrm{~K}+\mathrm{R})$ making up the protein, $\mathrm{pI}$ of the protein, and number of putative heparin binding sites found on the surface of the protein. 


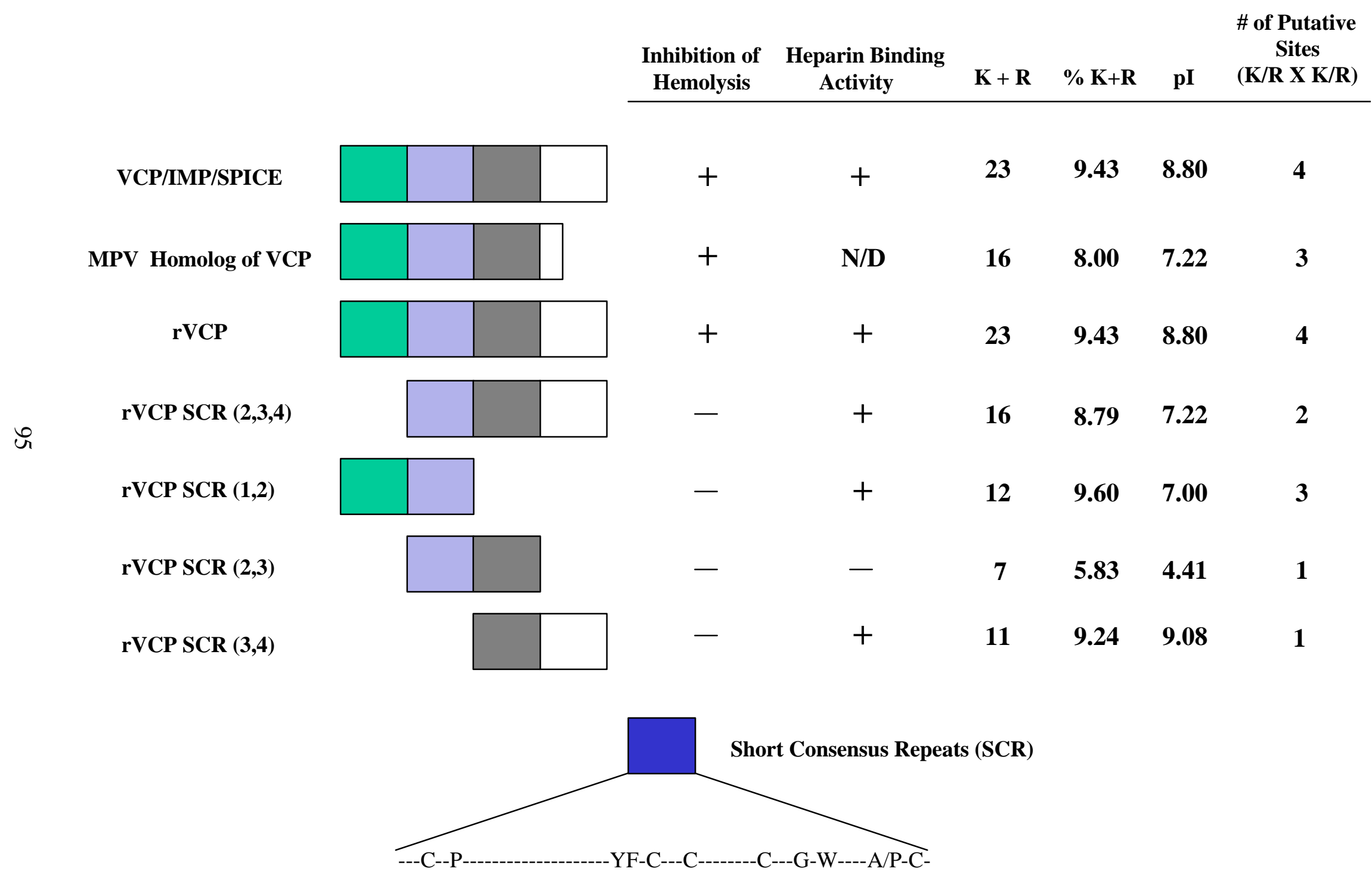




\section{Biacore Heparin Binding:}

To map the heparin binding site(s) on VCP using SPR, heparin was immobilized to a CM5 sensor chip and rVCP, or rVCP fragments, was injected and their ability to bind measured. As shown in figure 22 , both $\mathrm{rVCP}$ and $\mathrm{rVCP} \sim 3,4$ bound strongly to the immobilized heparin. Surprisingly, $\mathrm{rVCP} \sim 1,2$, although the protein was purified using heparin affinity chromatography, did not show the ability to interact with heparin immobilized to the sensor chip. The chromatography data suggests that two sites on rVCP, that are highly homologous to the well-characterized heparin binding site of fibroblast growth factor protein, may be involved in heparin binding. The two sites are located at the junction between CCP modules 1 and 2 (KRR), and at the far end of module $4(\mathrm{KQK})$. These sites were first identified when mutant proteins $\mathrm{rVCP} 1,2$ and rVCP 3,4 were shown to bind and be purified using heparin affinity chromatography. The second heparin binding site found at the junction of CCP modules 1 and 2 may possess a much weaker affinity, and without the presence of the remaining half of the protein is unable to bind heparin using SPR. This region may therefore double as a heparin/C3b-C4b binding site, binding weakly to heparin only in the absence of complement.

\section{Tri-peptide Studies:}

Small tri-peptides were ordered from Bio-Synthesis Incorporated (Lewisville, Texis) to determine whether the heparin binding of rVCP could be inhibited in the presence of a high concentration of putative, $\mathrm{K} / \mathrm{R}-\mathrm{X}-\mathrm{K} / \mathrm{R}$, binding sites. The putative heparin binding site, KRR, as well as control tri-peptides DID (negatively charged) and 
Figure 22. Heparin binding of rVCP fragments. The ligand and control channel responses are shown for the binding between $\mathrm{rVCP}$, or rVCP fragments, and heparin immobilized to the sensor chip. Control channel response represents only bulk effects; no protein is immobilized. All proteins were tested at different concentrations to confirm the presented results. 


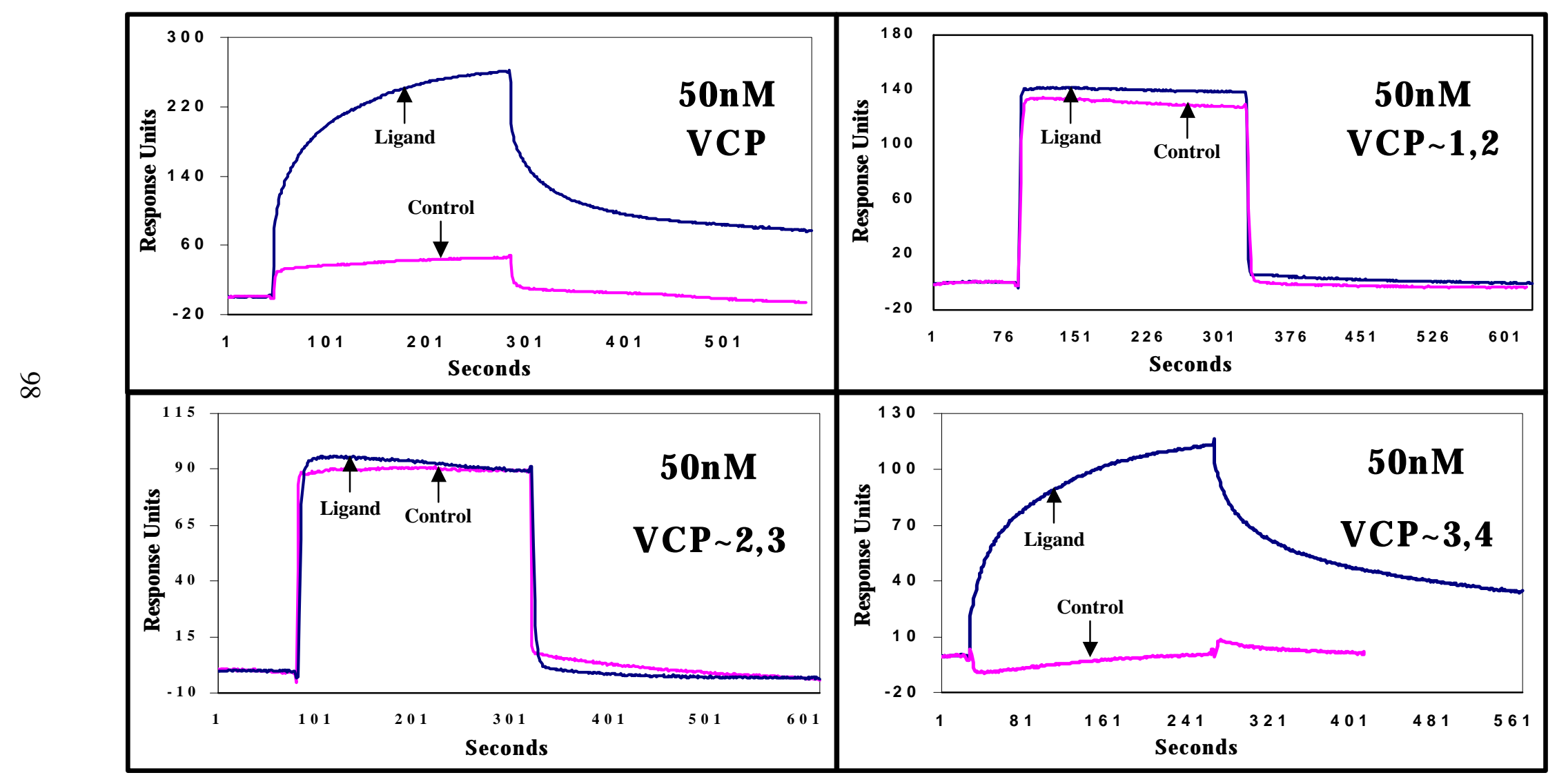


GSS (neutral), were each tested in various concentrations to see what effect they would have in rVCP's ability to bind heparin. As can be see in figure $23, \mathrm{rVCP}$ binding to heparin was actually enhanced with increasing concentrations of the KRR tri-peptide. This effect was also seen, though to a lesser extent at higher concentrations, with the DID and GSS tri-peptides - see figures 24 and 25. These results suggested that the KRR tripeptide might bridge the central negatively charged region of $\mathrm{VCCP}$, and thus increase its interaction with heparin by increasing its positive electrostatic field. If this hypothesis were true, $\mathrm{rVCP} 2,3$ (normally unable to bind heparin due to strong negative charge), in the presence of high concentrations of KRR, would bind heparin. As can be seen in figure 26, this was not the case. $\mathrm{rVCP} 2,3$ did not bind heparin even in the presence of high concentrations of the KRR tri-peptide.

\section{Dextran Sulfate Affinity Chromatography:}

As an alternative to heparin chromatography, in an attempt to reduce expense and increase binding capacity, dextran sulfate matrix was purchased from Sigma. Using a disposable $20 \mathrm{ml}$ Bio-Rad column, $20 \mathrm{ml}$ of sulfated dextran matrix was seeded and rinsed with water. Figure 27 shows the results obtained using a $\mathrm{NaCl}$ step gradient to elute rVCP. rVCP was found to bind dextran sulfate with a higher affinity $(500 \mathrm{mM}$ elution) than heparin (400 $\mathrm{mM}$ elution). In addition, its binding capacity seemed to be greater than that observed for heparin matrices. Unfortunately, however, the dextran sulfate matrix expanded too much to use for large-scale purification of rVCP. 
Figure 23. Heparin binding of $\mathbf{r V C P}$ in presence of KRR tri-peptide. Heparin affinity chromatography was performed on rVCP in the presence of varying amounts of the positively charged tri-peptide KRR. rVCP was eluted from the column using a $\mathrm{NaCl}$ gradient ranging from $100 \mathrm{mM}$ to $2 \mathrm{M}$. rVCP eluted in the $300 \mathrm{mM} \mathrm{NaCl}$ fraction in the absence of KRR, but eluted as high as $500 \mathrm{mM}$ in the presence of large concentrations of KRR. It is not known why the KRR tri-peptide was stained while the DID and GSS tripeptides (figures 24 and 25) were not. 
(KRR) Tri-peptide and rVCP Heparin Binding

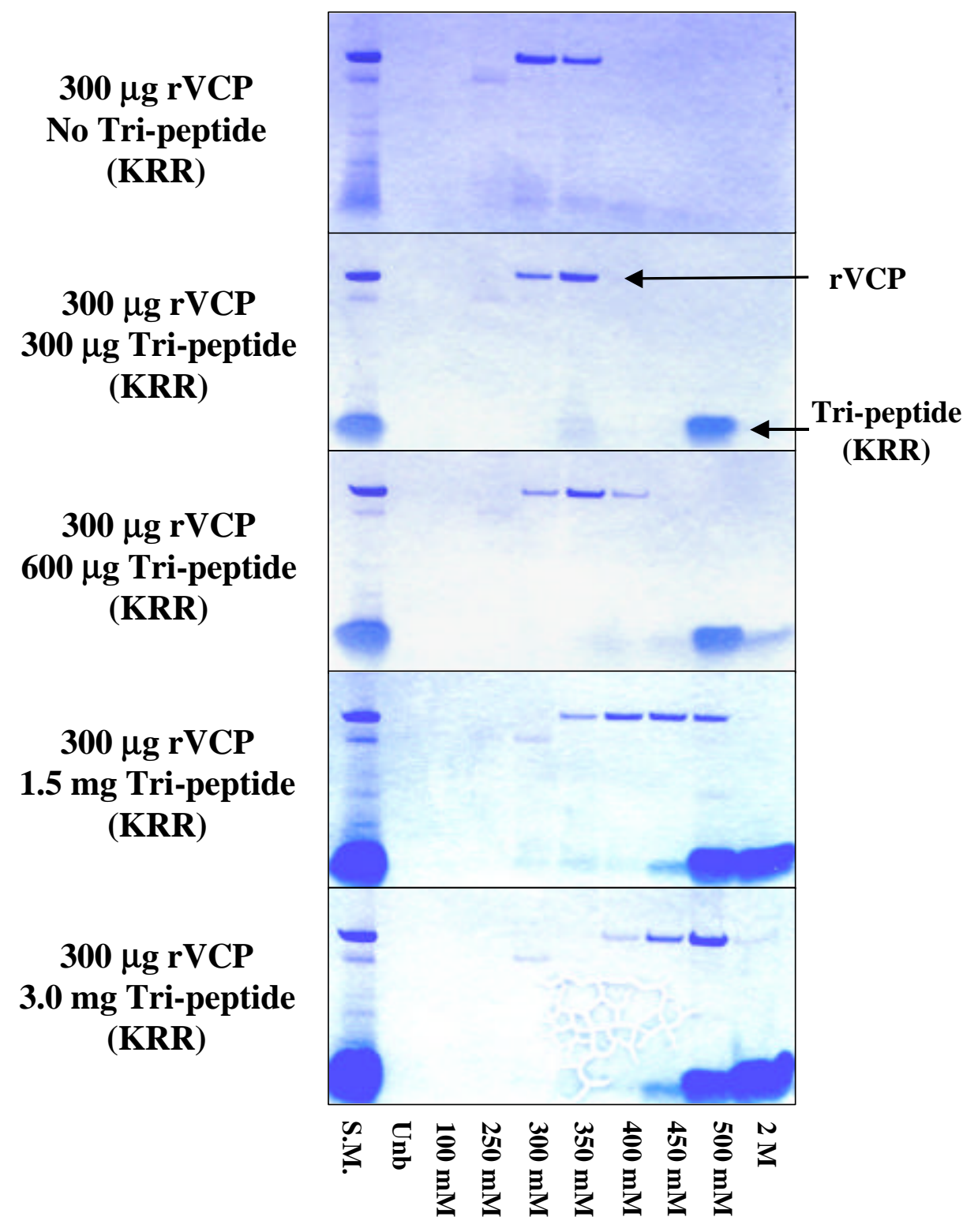


Figure 24. Heparin binding of $\mathbf{r V C P}$ in presence of DID tri-peptide. Heparin affinity chromatography was performed on rVCP in the presence of varying amounts of the negatively charged tri-peptide DID. rVCP was eluted from the column using a $\mathrm{NaCl}$ gradient ranging from $100 \mathrm{mM}$ to $2 \mathrm{M}$. $\mathrm{rVCP}$ eluted in the $300 \mathrm{mM} \mathrm{NaCl}$ fraction in the absence of DID, and eluted as high as $450 \mathrm{mM}$ in the presence of large concentrations of DID. Although DID resulted in rVCP having an increased affinity for heparin, this affinity was significantly lower at higher concentrations of tri-peptide than that observed in the presence of the KRR tri-peptide. 
(DID) Tri-peptide and rVCP Heparin Binding

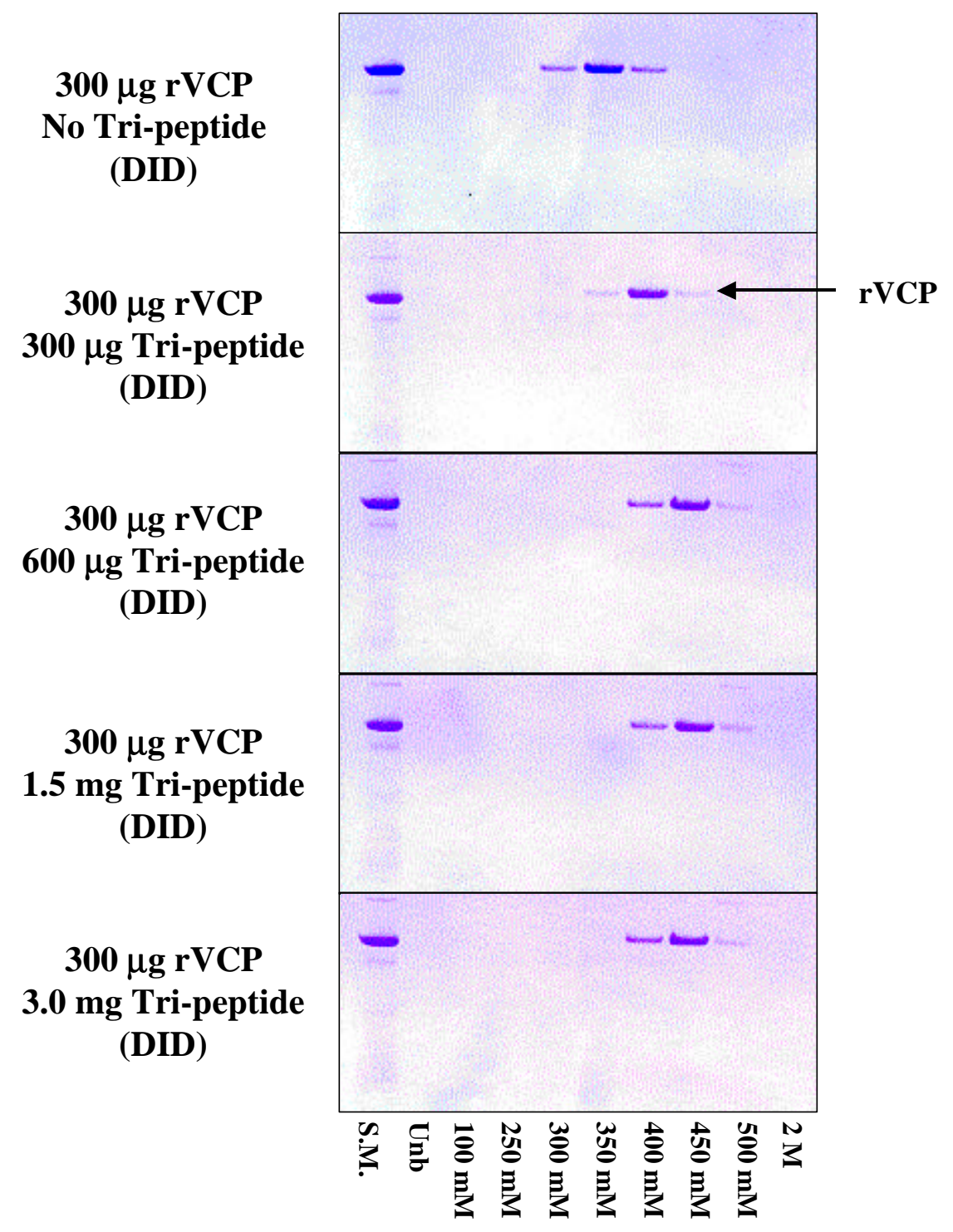


Figure 25. Heparin binding of rVCP in presence of GSS tri-peptide. Heparin affinity chromatography was performed on rVCP in the presence of varying amounts of the neutrally charged tri-peptide GSS. rVCP was eluted from the column using a $\mathrm{NaCl}$ gradient ranging from $100 \mathrm{mM}$ to $2 \mathrm{M}$. $\mathrm{rVCP}$ eluted in the $300 \mathrm{mM} \mathrm{NaCl}$ fraction in the absence of GSS, and eluted as high as $450 \mathrm{mM}$ in the presence of large concentrations of GSS. Although GSS resulted in rVCP having an increased affinity for heparin, this affinity was also significantly lower than that observed in the presence of the KRR tripeptide at the $1.5 \mathrm{mg}$ tri-peptide concentration. 
(GSS) Tri-peptide and rVCP Heparin Binding

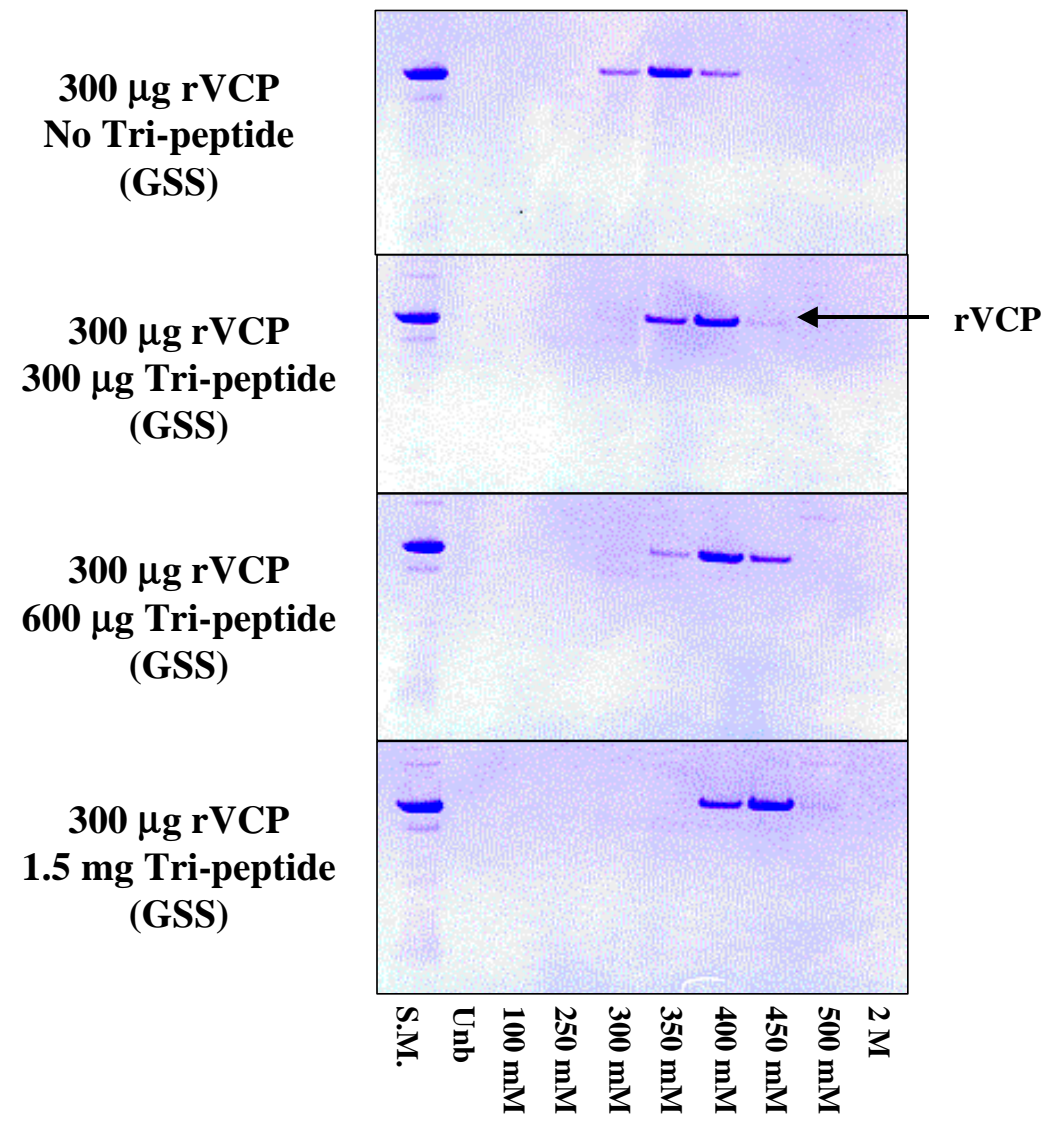


Figure 26. Heparin purification of $\operatorname{rVCP} \sim 1,2, \operatorname{rVCP} \sim 2,3$ (using KRR), rVCP 2,3,4. Purification of $\mathrm{rVCP} \sim 1,2, \quad \mathrm{rVCP} \sim 2,3$, and $\mathrm{rVCP} \sim 2,3,4$ using heparin affinity chromatography. $\mathrm{rVCP} \sim 1,2$ and $\mathrm{rVCP} \sim 2,3,4$ are easily purified by this method due to their strong affinity for heparin, however, $\mathrm{rVCP} \sim 2,3$ does not show this affinity and must be purified by other means. Attempts were made to use the tri-peptide KRR to purify rVCP 2,3 by heparin affinity chromatography, but failed. Proteins were eluted using a $\mathrm{NaCl}$ step gradient ranging from $100 \mathrm{mM}$ to $2 \mathrm{M}$. 


\section{Heparin Purification of rVCP $(1,2),(2,3),(2,3,4)$}

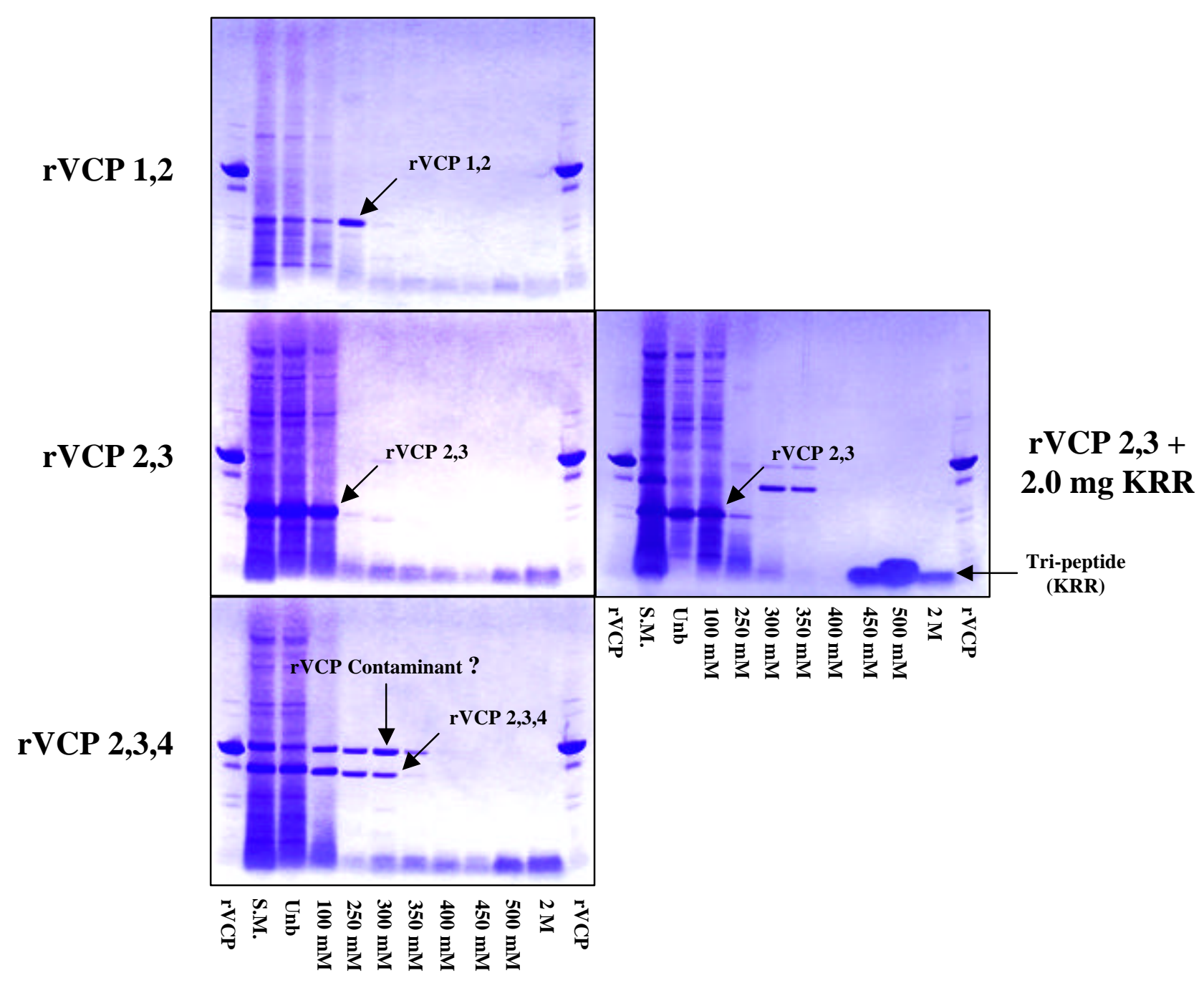


Figure 27. Dextran sulfate chromatography of rVCP. Do to the similarity between dextran sulfate and heparan sulfate, attempts were made to purify rVCP by dextran sulfate chromatography. $\mathrm{rVCP}$ was eluted using a $\mathrm{NaCl}$ step gradient ranging from 300 $\mathrm{mM}$ to $1 \mathrm{M}$. Interestingly, rVCP eluted from the dextran column at a significantly higher $\mathrm{NaCl}$ concentration (500 $\mathrm{mM}$ as compared to $350-400 \mathrm{mM}$ ) than seen using heparin chromatography. This indicates that rVCP possesses a higher affinity toward dextran sulfate than heparin. This may be due to the greater degree of sulfation present on the dextran sulfate molecule. 


\section{Dextran Sulfate Purification of rVCP}

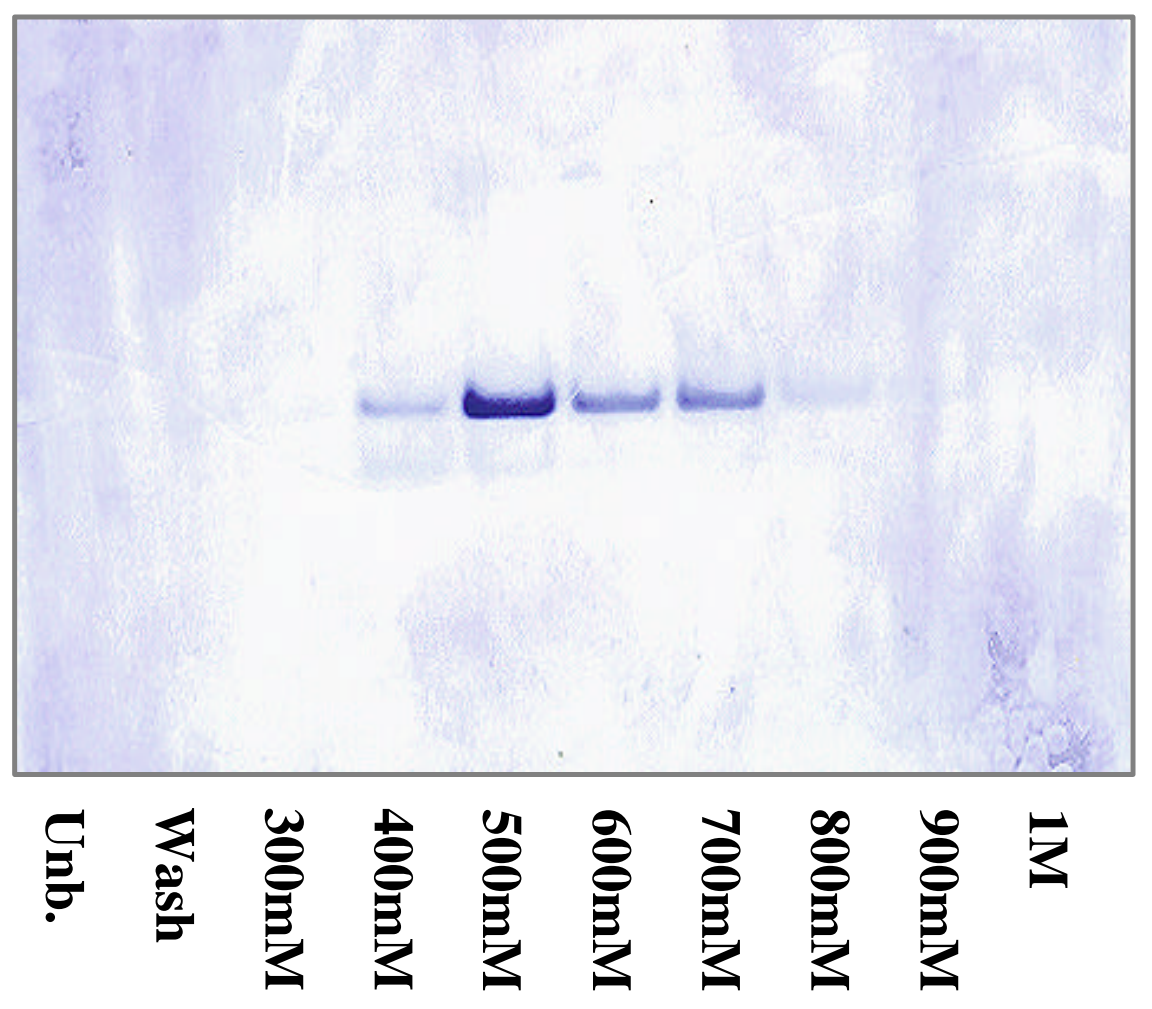




\section{NMR Modeled Structure:}

In order to better visualize the positioning of heparin binding sites in the VCP molecule, a model was developed using four known VCP module structures: VCP SCR 3, VCP SCR 4, factor H (fH) SCR 15 and fH SCR 16 - see figure 28. As can be seen in this model, heparin binding exists primarily at the ends - SCRs one and four. The middle of the molecule therefore does not contribute any to the binding (hence $\mathrm{rVCP} 2,3$ showed no ability to bind).

\section{Electrostatic Charge Distribution:}

Using the X-ray crystal structure of rVCP, the electrostatic field distribution was predicted. An extreme positively charged field over the fourth domain is very evident. In addition, concentration of basic residues [Lys $(\mathrm{K})$ and $\operatorname{Arg}(\mathrm{R})$ residues] at the junction of modules 1 and 2 makes a second highly positively charged patch - see figure 29. Most of the residues with carboxylic acid side chains are clustered in modules 2 and 3 with those two modules carrying a net negative charge.

\section{$\underline{X-R a y ~ C r y s t a l ~ S t r u c t u r e ~ H o m o l o g y: ~}$}

Other than VCP, only the serum complement regulatory proteins $\mathrm{C} 4 \mathrm{~b}-\mathrm{BP}$ and $\mathrm{fH}$ have heparin binding activity. Binding sites for heparin on CCP modules 1 and 2 on the $\alpha$ chain of C4b-BP (Blom et al., 1999; Hessing et al., 1990) and on CCP modules 7, 20 and 13 for fH (Blackmore et al., 1998; Sharma and Pangburn, 1996) have been identified. The above experimental results suggest the possible presence of two heparin binding sites on modules 1 and 4 of rVCP. 
Figure 28. VCP model showing the heparin binding sites. Front (A) and back (B) views of the modeled structure of VCP SCR (1-4) showing the heparin binding sites (differentially colored). In order to differentiate the extents of the individual modules, they are shaded appropriately. 

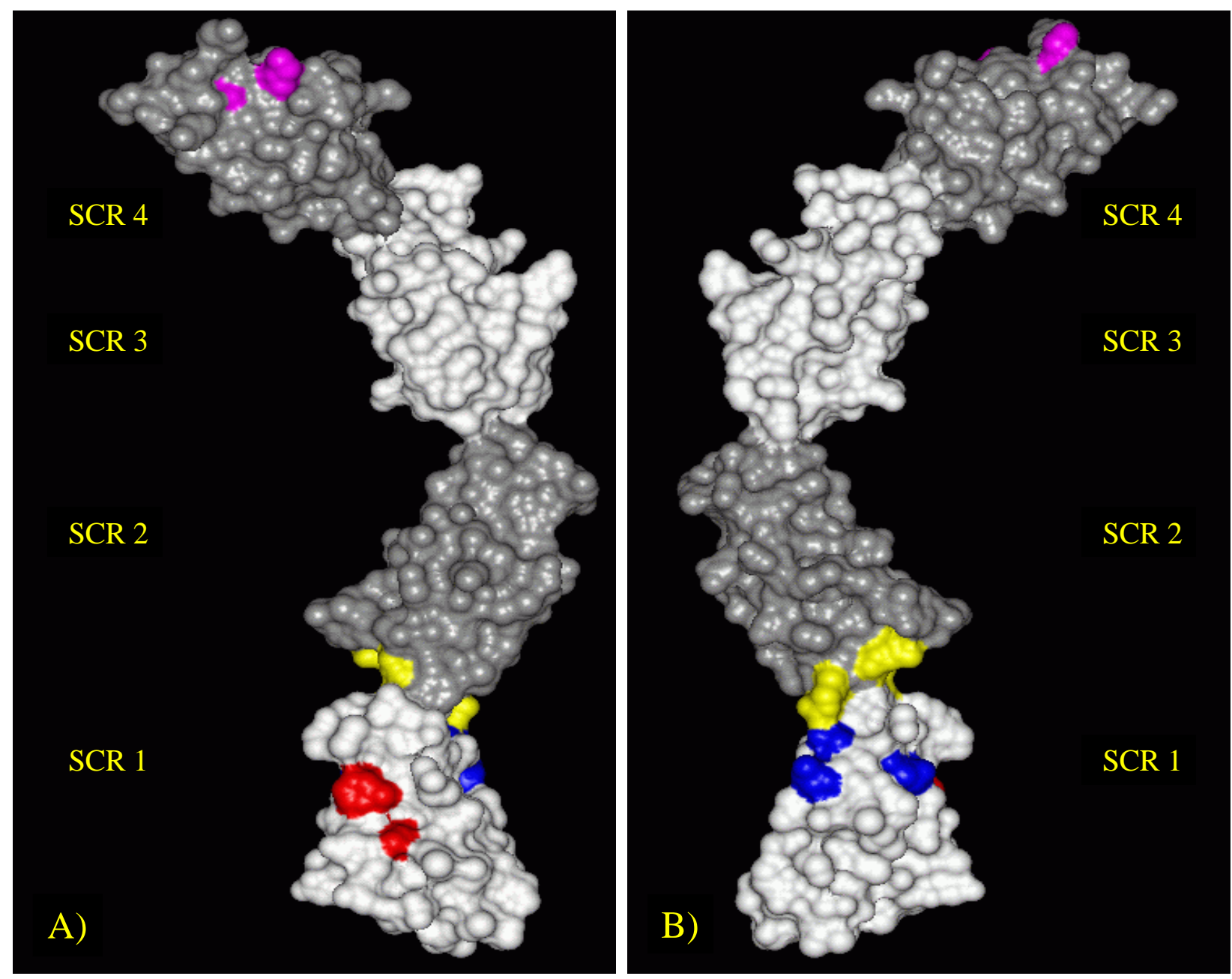
Figure 29. Electrostatic attributes of VCP. Domain 1 is at the top and domain 4 at the bottom in all panels. a-d) Electrostatic potential mapped on to the molecular surface of molecule A of form I crystals. Dark blue represents 10.2 electrons per $\AA$ and red represents -4.3 electrons per $\AA$. e) Representation of the $\pm 2 \mathrm{kT}$ contour of the potential field around the molecule calculated and displayed by GRASP using program defaults for all parameters, $\alpha$-carbon trace is shown in yellow. f) Expanded views of the two positive patches between domains 1 and 2 (top) and domain 4 (bottom). The top half of the panel is in approximately the same orientation as panel a; the bottom panel represents a view as seen from the bottom of panel a looking up at module 4 . $\mathrm{K}$ and $\mathrm{R}$ residues contributing to each patch are labeled. For the primary structure of rVCP, see figures 20 and 57. 

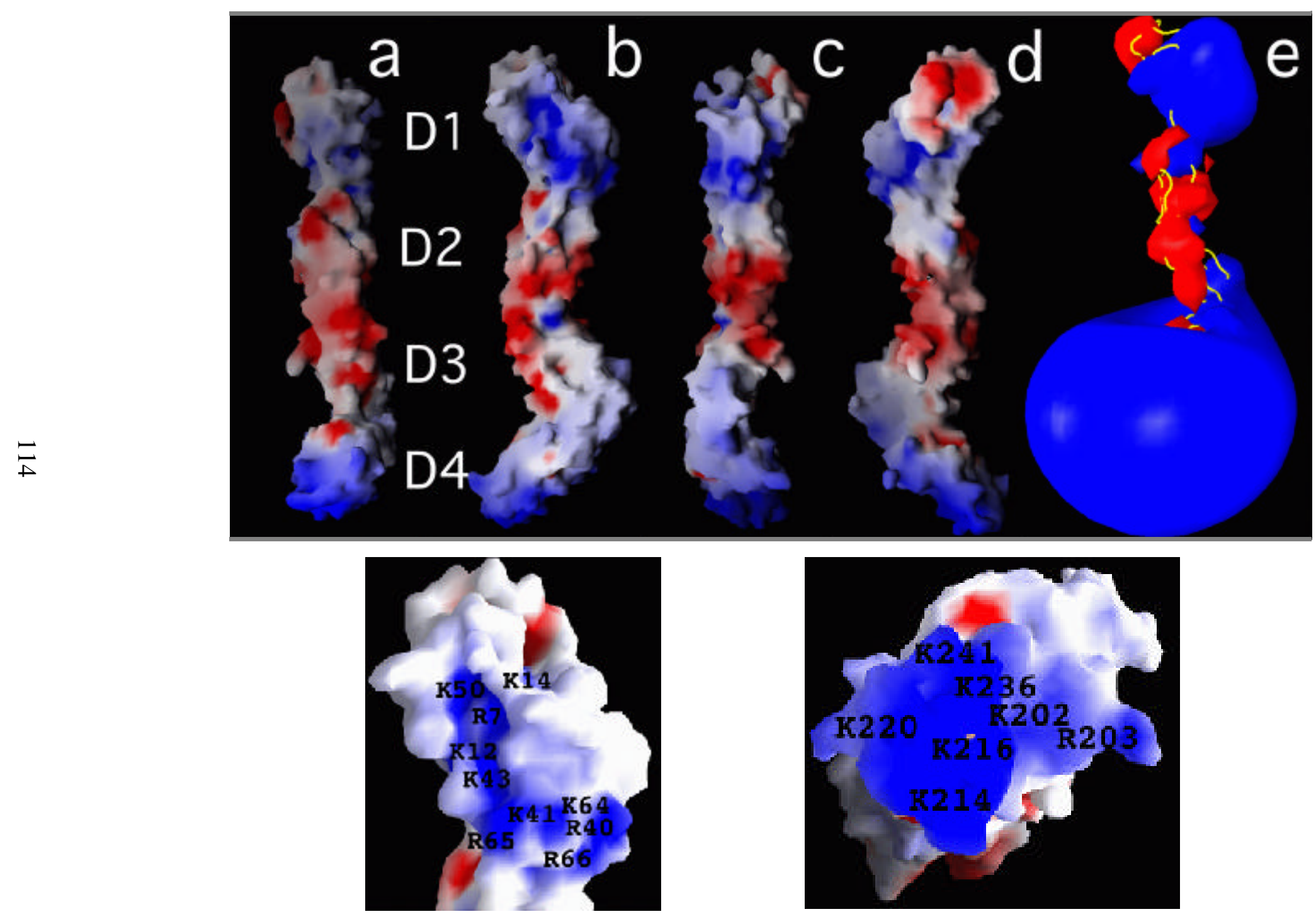
The structure of VCP confirms that there are two spatially distinct putative heparin binding sites in the molecule. rVCP, in the crystal structures, appears to be a long molecule with large positively charged domains at its two ends; a large patch of positive charge is located at the tip of domain 4 and a second positive patch straddles domains 1 and 2. Negatively charged groups on heparin and positively charged groups on proteins dominate heparin-protein interactions. Heparin binding sequence motifs in proteins consisting of suitably spaced $\mathrm{K}$ and $\mathrm{R}$ residues have been proposed (Margalit $e t$ al., 1993) and such motifs have been identified in VCP. Structures of acidic (DiGabriele et al., 1998; Schlessinger et al., 2000) and basic (Faham et al., 1996) fibroblast growth factors (FGFs) complexed with heparin have offered insights into details of binding of heparin by proteins. Modeling of heparin binding to rVCP employing FGF as a template and using Insight II (Biosym-Technologies, 2000) shows that $\alpha$ carbon atoms of $\mathrm{K}$ residues, 214, 216, 220 and 241, in a flexible region of module 4, can be superimposed on corresponding basic residues, K113, R119, R112 and K128 in acidic FGF (DiGabriele et al., 1998; Schlessinger et al., 2000). All the ligands in module 4 are either end residues of $\beta$ strands or are in flexible loops between strands - see figure 30. Similarly, residues K64, R65 and R66 in module 1 and the linker connecting modules 1 and 2 can be superimposed on residues K122, K118 and R119 of FGF. These two putative heparin binding regions are separated by about $80 \AA$. Figure 30 shows a stereo view of the model for the site at the tip of module 4. It is significant that the model for rVCP interactions with heparin resembles that in the FGFs closely, an unrelated protein, indicating heparinprotein recognition mode of wide applicability. 
Figure 30. Stereo view of interaction of VCP with Heparin. An C $\alpha$ trace of residues 213-242 of VCP is shown in orange. Carbohydrate is shown with carbons colored gray, oxygen, red, nitrogen blue and sulfur yellow. Sidechains of the four basic residues of acidic and basic FGF and VCP that were superimposed are shown in red, magenta and cyan respectively. VCP sidechains are labeled. The figure was composed using a rigid body transformation of VCP mainchain on to the basic FGF heparin binding segment. The sidechains of the $\mathrm{K}$ residues in VCP have been rotated to point towards the heparin fragment. The side chain of K 214 is beyond hydrogen bonding distance from heparin as currently modeled. Mainchain movements of $1-2 \AA$ will be required to bring its sidechain within interacting distance. 


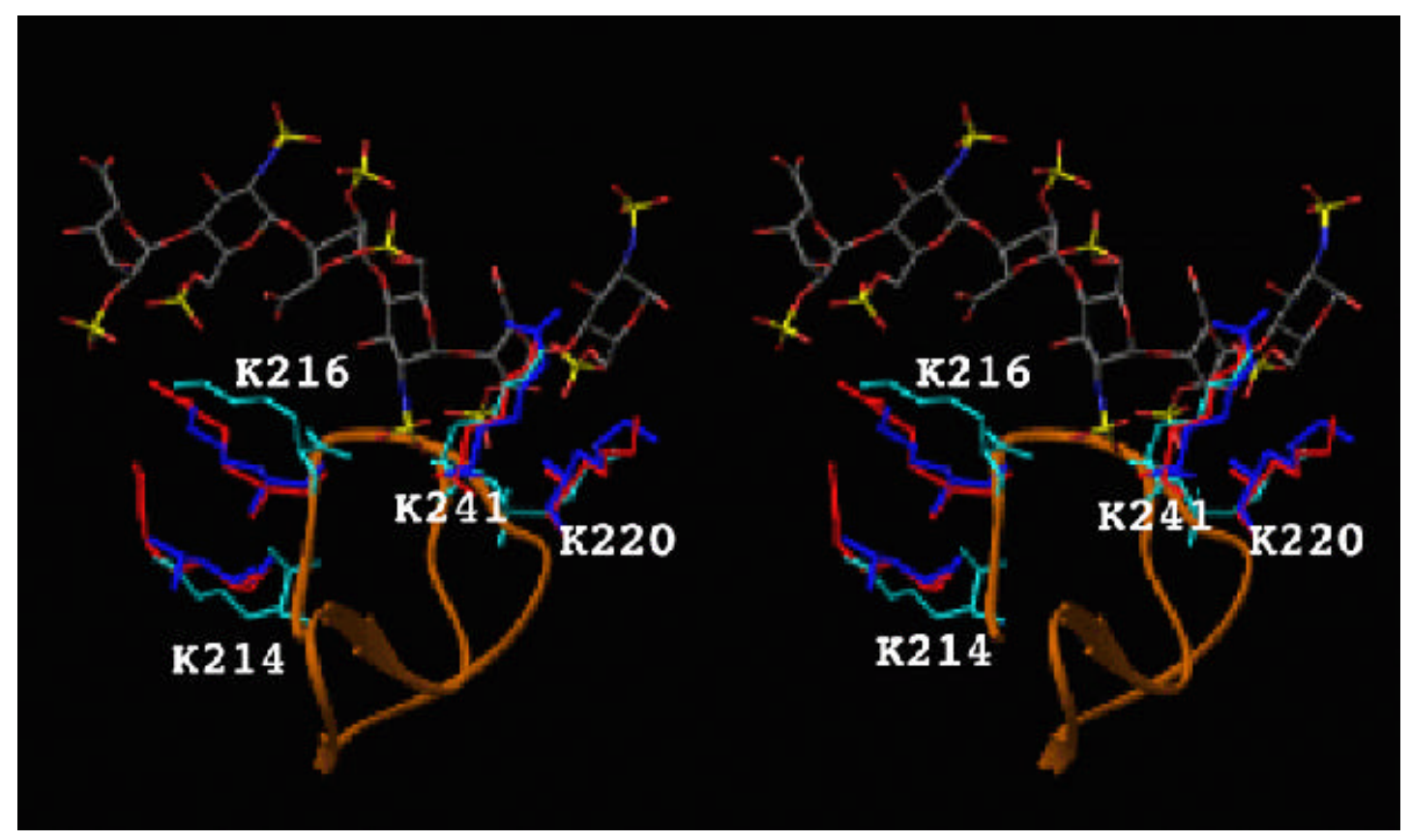




\section{$\underline{\text { Discussion }}$}

The ability of VCP to bind heparan sulfate proteoglycan molecules adds a new dimension to its role in modulating the host immune response. In previous studies, the ability of VCP to bind heparin was found to permit uptake by mast cells - possibly allowing for tissue persistence over an extended time. In addition, earlier studies have shown that binding to heparin-like molecules on the surface of endothelial cells can block chemokine adherence, resulting in reduced chemotactic signaling. The next step was to determine if VCP, bound to the endothelial cell surface, could interfere with antibody binding. In this study, flow cytometry was used to demonstrate that VCP is able to bind the surface of endothelial cells, interfering with antibody binding. More importantly, this study demonstrates that addition of VCP significantly decreases the amount of antibody able to attach to HUVECs in a dose-dependent manner. It is postulated that VCP, binding to heparin-like molecules on the surface of endothelial cells, prevents antibody adherence. This binding may therefore block antibody attachment through steric interference, and block both molecular and cellular interactions - which are dependent on target cell adhesion molecules binding to cytotoxic cells. Furthermore, this novel mechanism can enable virus-infected cells or the antigen presenting cell from destruction by host defense mechanism. Interestingly, it was estimated that the amount of VCP required for inhibition of roughly $5 \times 10^{5}$ cells is approximately the same as that produced by $2 \times 10^{5}$ infected cells, and therefore each infected cell can protect itself and one or two surrounding cells. Thus, VCP enables orthopoxviruses, all of which encode homologues of VCP, to evade the immune system by multiple mechanisms, e.g. by blocking complement pathways, blocking antibody-dependent cytotoxic cell activity 
(ADCC) or by interfering with attachment of cytotoxic T cells to virus infected cells. This observation is further strengthened by recent studies by Al-Mohanna et al. (2001), who showed that rVCP was able to block interactions between pig aortic endothelial cells (PAECs) and naïve neutrophils and NK cells, preventing cell killing (Al-Mohanna et al., 2001). In addition, cell culture studies demonstrate that VCP blocks complementmediated killing of PAECs by human serum, in a dose-dependent manner.

Due to the apparent importance heparin binding has in many of the biological activities exhibited by VCP, we became interested in better characterizing the molecular reasons for this interaction. Recombinant segments of VCP - each representing two of the four SCRs - were generated by genetic engineering vaccinia virus DNA, encoding VCP, into the Pichia pastoris expression system. The ability of these recombinant segments to bind heparin was then tested. It was determined that recombinant proteins $\mathrm{rVCP} \sim 1,2$ and $\mathrm{rVCP} \sim 3,4$ retained the same binding affinity as the full-length native protein. While recombinant protein $\mathrm{rVCP} 2,3$ retained no heparin binding ability at all. In order to explain this, the amino acid sequences of the recombinant protein segments were more closely examined. It was determined that the number, and more importantly, the percentage of positively charged amino acids making up the protein contributed greatly to its ability to interact with heparin. In addition, the overall charge and number of putative binding sites $(\mathrm{K} / \mathrm{R}-\mathrm{X}-\mathrm{K} / \mathrm{R})$ making up the protein, appears to play a role in heparin binding. For the most part, the ability of VCP to bind heparin lies in its first and fourth SCR. These two short consensus repeats possess within them the highest percentage of positive amino acids, the greatest number of putative binding sites, and have a very strong overall positive charge (data not shown). 
Using SPR, Biacore binding analysis, the ability of rVCP and rVCP fragments was tested in order to confirm the locations of the heparin binding sites. The two sites are located at the junction between CCP modules 1 and 2, and at the far end of module 4 . These sites were first identified in the above experiments where mutant proteins $\mathrm{rVCP} \sim 1,2$ and $\mathrm{rVCP} \sim 3,4$ are found to bind and be purified using heparin affinity chromatography. With heparin immobilized to the sensor chip, rVCP and the various mutant rVCP truncations were injected and their interaction measured. rVCP and rVCP 3,4 was found to bind strongly to the immobilized heparin. Surprisingly, rVCP 1,2 did not show the ability to interact with heparin immobilized to the sensor chip. The second heparin binding site found at the junction of CCP modules 1 and 2, may possess a much weaker affinity and without the presence of the remaining half of the protein is unable to bind heparin using SPR. It is now believed that this region may double as a heparin/C3b-C4b binding site, binding weakly to heparin only in the absence of complement.

In order to better visualize the positioning of heparin binding sites in the VCP molecule, a model was developed using four known VCP module structures: VCP SCR 3, VCP SCR 4, factor H (fH) SCR 15 and fH SCR 16. As can be seen in this model, heparin binding exists primarily at the ends - SCRs one and four. The middle of the molecule therefore does not contribute any to the binding (hence $\mathrm{rVCP} \sim 2,3$ showed no ability to bind). This model was confirmed when the crystal structure was resolved. Using the crystal structure, an accurate assessment of charge distribution could be used to authenticate the heparin binding site locations. Indeed, strong positive charge can be seen surrounding the locations of the heparin binding sites at the junction between CCP 
modules 1 and 2, and at the far end of module 4. In addition, using spatial modeling, both of these regions showed a considerable degree of homology with the heparin binding site of acidic and basic fibroblast growth factor (FGF) protein. The small heparin binding sequence of FGF is often used to define heparin binding sites on other proteins. Due to the very close alignment of critical basic amino acid residues on rVCP with FGF, it is almost certain that these two putative heparin sites are responsible for VCP's ability to interact with heparin.

Using synthetic tri-peptides to mimic the short conserved sequences (K/R-X-K/R) suspected to be involved in the heparin binding of rVCP, competition analysis was performed. The ability of rVCP to bind heparin was actually enhanced in the presence of increasing concentrations of the predicted heparin binding site sequence. This did not prove that these sequences were not involved in heparin binding, although it suggested that the sequences were not this size. Previously published heparin binding sites are much longer than three amino acids and containing spacer regions to spatially position the basic amino acids in alignment with the negatively charged heparin. All of the above data suggests that the $\mathrm{K} / \mathrm{R}-\mathrm{X}-\mathrm{K} / \mathrm{R}$ binding motif is, in fact, part of the heparin binding sites of $\mathrm{rVCP}$, but is not the entire site required for the interaction. It is now believed that the heparin binding site at the junction between CCP modules 1 and 2 does consist of amino acids K64, R65, and R66, (and possibly R40, K41, and K43) but does however involve the linker region between CCP 1 and 2 as well. Likewise, a series of $\mathrm{K}$ residues, 214, 216, 220 and 241, which also incorporates a K/R-X-K/R binding motif, makes up the second heparin binding site on a flexible region at the tip of module 4 - see figure 31 . 
Figure 31. Residues involved in binding heparin in VCP. Spatial relationship of two heparin binding sites on VCP. Residues K64, R65, and R66 (cyan) between modules 1 and 2 are believed to be involved in weak heparin binding in the absence of complement, image A. In the presence of complement, these basic residues are thought to double as a ligand for C3b/C4b, image B. Residues K214, K216, K220, and K241 (cyan) at the Cterminus of VCP are thought to bind heparin strongly, contributing significantly to VCP's heparin binding abilities. Heparin hexasaccharide (carbon, white; oxygen, red; nitrogen, blue; and sulfur, yellow) is modeled in A and B, with the four lysine residues which likely act as ligands. 

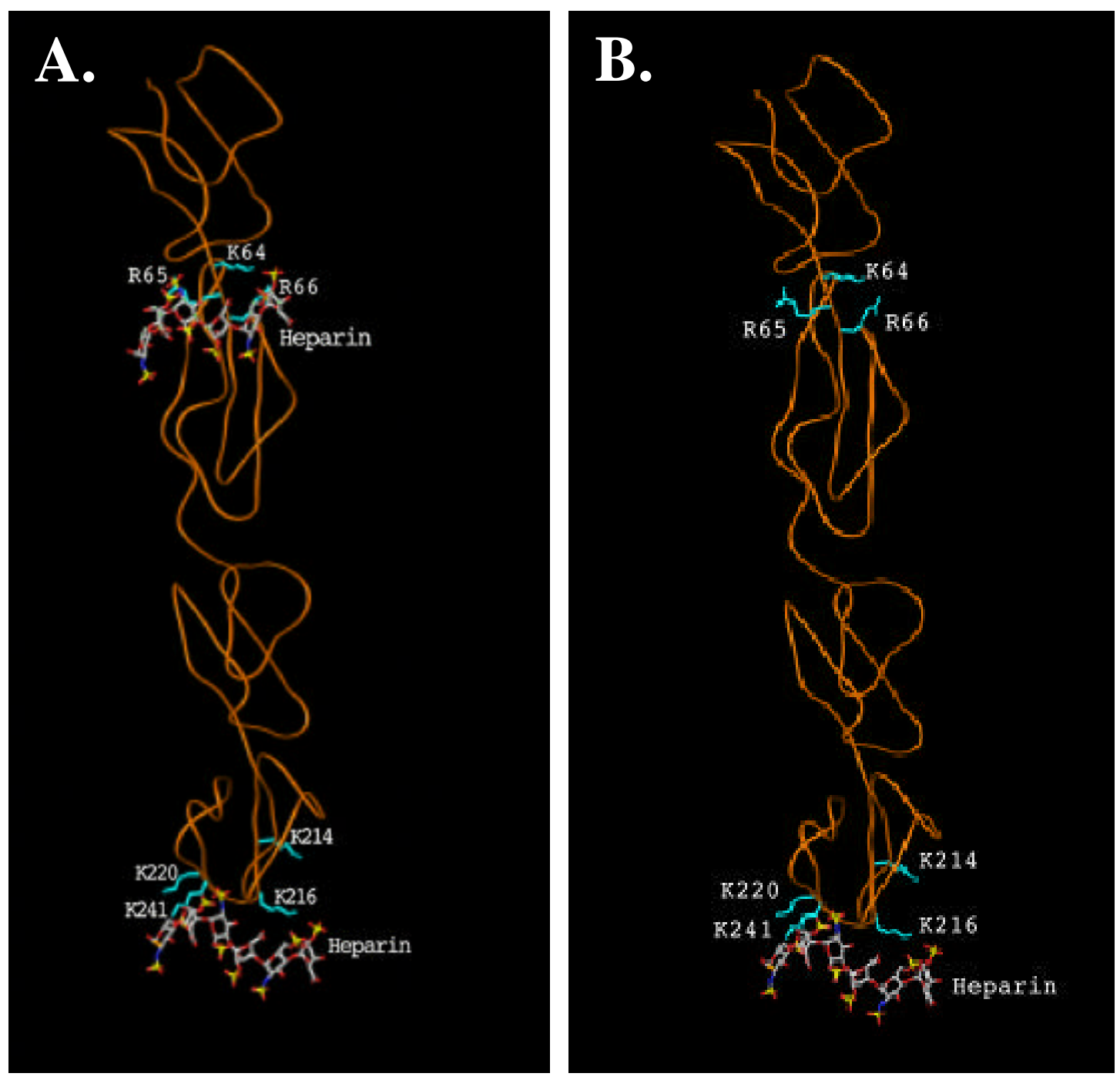
The importance of heparin binding to the function of soluble complement control proteins is becoming more evident. Studies of factor $\mathrm{H}(\mathrm{fH})$ show that heparin binding is very important in distinguishing activator surfaces from host cell surfaces (Pangburn et al., 2000). In addition, both C3b decay acceleration and co-factor activities of $\mathrm{fH}$ are enhanced in the presence of heparin (Meri and Pangburn, 1994). This may add strength to the hypotheses that VCP binds heparan sulfate on the surface of vaccinia virus infected host cells to protect the cell from complement activation, cell lysis, and thus preserves the viral habitat.

In summary, by examining the heparin-binding ability of VCP, we have gained a better understanding of the molecular basis by which this interaction occurs. In addition, the data presented here further enhances our understanding of this truly unique, yet compact, multifunctional viral protein. It resembles in structure and function giant complex human complement regulatory proteins human $\mathrm{C} 4 \mathrm{~b}$ binding protein $(\mathrm{C} 4 \mathrm{~b}-\mathrm{BP})$ and the complement receptor 1 (CR1), respectively. Not only is VCP a miniature version of these large molecules, it has retained all the complement modulating functions, and as we have described here, added a number of additional functions to its repertoire due to its ability to bind heparin-like molecules. This better understanding may some day help in the exploitation of VCP's possible therapeutic properties in the treatment of inflammatory conditions such as Alzheimer's Disease (Daly and Kotwal, 1998), CNS injury, systemic lupus erythematosus, and xenograft transplant rejections. 


\title{
CHAPTER 4
}

\section{COMPLEMENT BINDING ACTIVITY OF VCP}

\author{
Introduction to Complement Binding
}

This chapter contains material adapted from the following publications and manuscript:

Smith S.A., N.P. Mullin, J. Parkinson, S.N. Shchelkunov, A.V. Totmenin, V.N. Loparev, R. Srisatjaluk, D.N. Reynolds, K.L. Keeling, D.E. Justus, P.N. Barlow, and G.J. Kotwal. Conserved surface-exposed K/R-X-K/R motifs and net positive charge on poxvirus complement control proteins serve as putative heparin binding sites and contribute to inhibition of molecular interactions with human endothelial cells: a novel mechanism for evasion of host defense. J. Virol. Jun. 74(12):5659-66, 2000 [Cover of J. Virol. Oct. 74(20), 2000].

Murthy K.H., Smith S.A., Ganesh V.K., Judge K.W., Mullins N., Barlow P.N., Ogata C.M., and Kotwal G.J. Crystal structure of a complement control protein that regulates both pathways of complement activation and binds heparan sulfate proteoglycans. Cell. Jan. 26, 104:301-11, 2001.

Smith S.A., Arjunwadkar S., Krishnasamy G., Judge K., Murthy K.H., Pugh D.R., and Kotwal G.J. Mapping of regions within the vaccinia virus complement control protein involved in binding to key complement components and heparin using surface plasmon resonance. Submitted to Virology, 2002.

In order to modulate the inflammatory response mounted by the host, many poxviruses encode proteins that mimic components of the complement and chemokine systems (Kotwal, 2000). Our lab has been interested in the many properties of the vaccinia virus complement control protein (VCP). Functionally, VCP is very similar to factor $\mathrm{H}(\mathrm{fH})$, membrane cofactor protein (MCP), decay-accelerating factor (DAF), and complement receptor one (CR1). Structurally, it is composed of four complement control protein (CCP) modules, also known as short consensus repeats (SCR) or Sushi domains, 
making it along with homologous proteins members of the complement control protein or regulators of complement activation (RCA) family - see figure 32 (Kotwal and Moss, 1988). VCP has been shown to inhibit the classical and alternative pathways of complement activation, through its ability to bind $\mathrm{C} 3 \mathrm{~b}$ and act as a cofactor for factor I cleavage, preventing the formation of the alternative pathway $\mathrm{C} 3$ and classical pathway C5 convertase (McKenzie et al., 1992; Kotwal et al., 1990; Sahu et al., 1998). VCP is also able to bind $\mathrm{C} 4 \mathrm{~b}$, allowing it to block early steps in the classical pathway of complement activation - see figure 33. By blocking complement activation at multiple sites, VCP down-regulates $\mathrm{C} 3 \mathrm{a}, \mathrm{C} 4 \mathrm{a}$, and $\mathrm{C} 5 \mathrm{a}$ proinflammatory chemotactic factors, resulting in reduced cellular influx and inflammation. Experimental support for this comes from in vivo experiments using BALB/c mice injected in the footpad with cowpox virus (CPV) expressing or lacking the VCP homologue in CPV, termed the inflammation modulatory protein (IMP) (Miller et al., 1997). A significantly greater influx of inflammatory cells into the tissue was observed when the footpad was infected with CPV lacking IMP as compared to that found in IMP expressing CPV. Another experiment, using $\mathrm{BALB} / \mathrm{c}$ congenically matched $\mathrm{C} 5$-sufficient and $\mathrm{C} 5$-deficient mice injected in the footpad with CPV, showed that C5-deficient mice exhibited a significantly greater swelling response that persisted longer and also showed signs of hemorrhage, edema, and ulceration (Miller et al., 1995). In addition, the presence of IMP drastically diminished the inflammatory response elicited by CPV (Kotwal et al., 1998a; Howard et al., 1998). As mentioned in chapter 1, the origin of vaccinia virus remains unknown. Since VCP is secreted from the infected host cell in order to establish a suitable habitat for viral replication, it seems likely that VCP may have evolved to be host specific. This means 
Figure 32. Several members of the complement control protein (CCP) family. Illustrated are the cartoon structures of VCP, C4b-BP, and CR1. These are examples of proteins that belong to the family of SCR containing proteins, also known as the $\mathrm{CCP}$ family. These proteins are made of repeating units, SCRs, which have a common, relatively conserved sequence, which results in two disulfide bonds and a characteristic sushi structure. The size of VCP, and the MPV homologue, is compared to that of C4b$\mathrm{BP}$, which it shares the highest degree of sequence homology, and CR1, which it shares the greatest functional homology. 


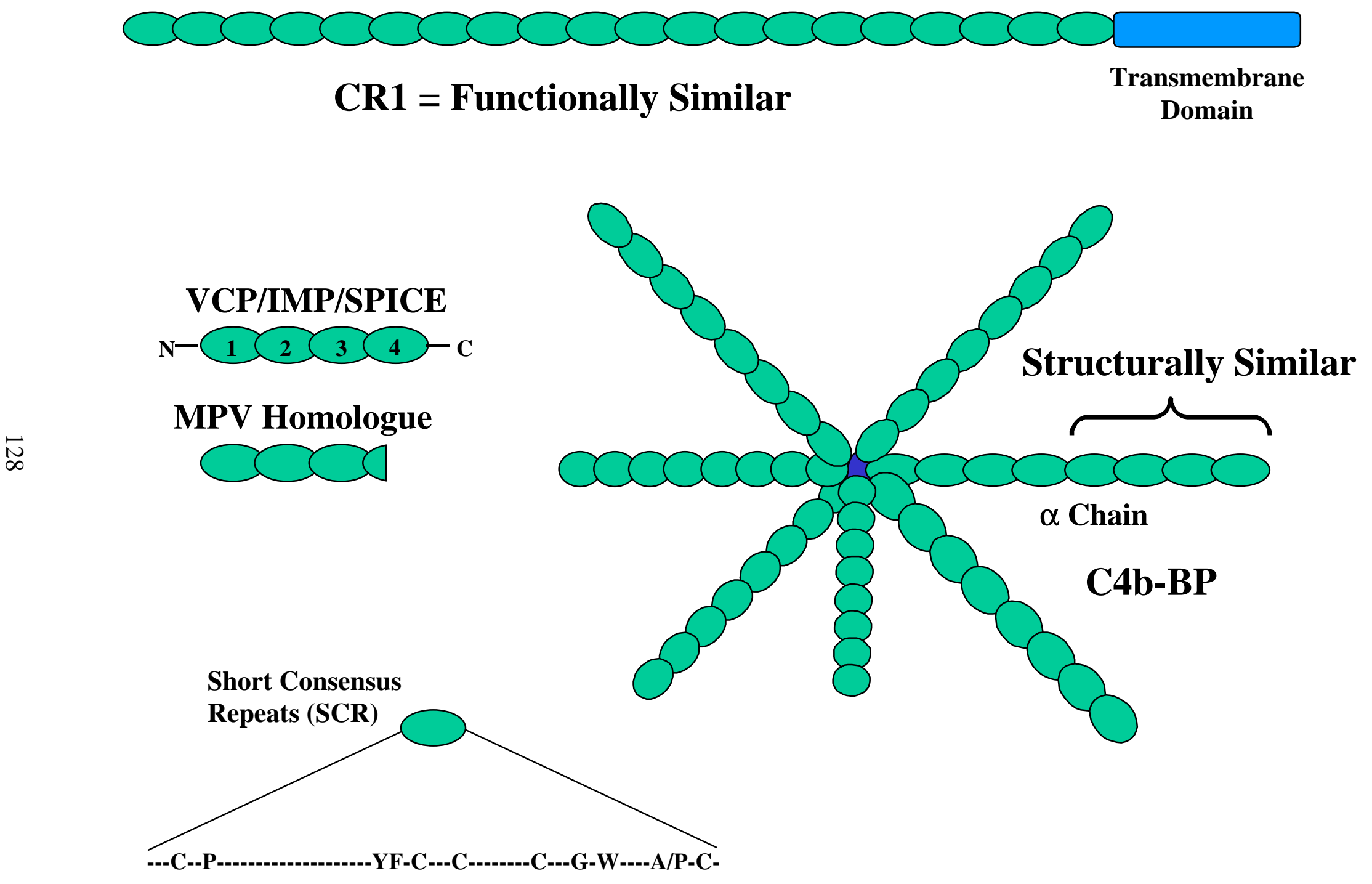


Figure 33. Complement cascade and points of VCP inhibition. Illustrated are the two main pathways of complement activation, the classical pathway, initiated by antibody fixation, and the alternative pathway, initiated by an activator surface. VCP possesses the ability to block both pathways of complement activation by binding $\mathrm{C} 3 \mathrm{~b}$ and $\mathrm{C} 4 \mathrm{~b}$ (depicted by red Xs), resulting in their cleavage and inactivation by factor I. This prevents the formation of the classical and alternative pathway C3 convertase, ultimately blocking activation at an early step. In addition, VCP blocks the formation of the C5 convertase in the classical pathway. By blocking multiple steps of complement activation, VCP is also able to prevent the release of proinflammatory chemotactic factors, such as, C4a, C3a, and C5a. 


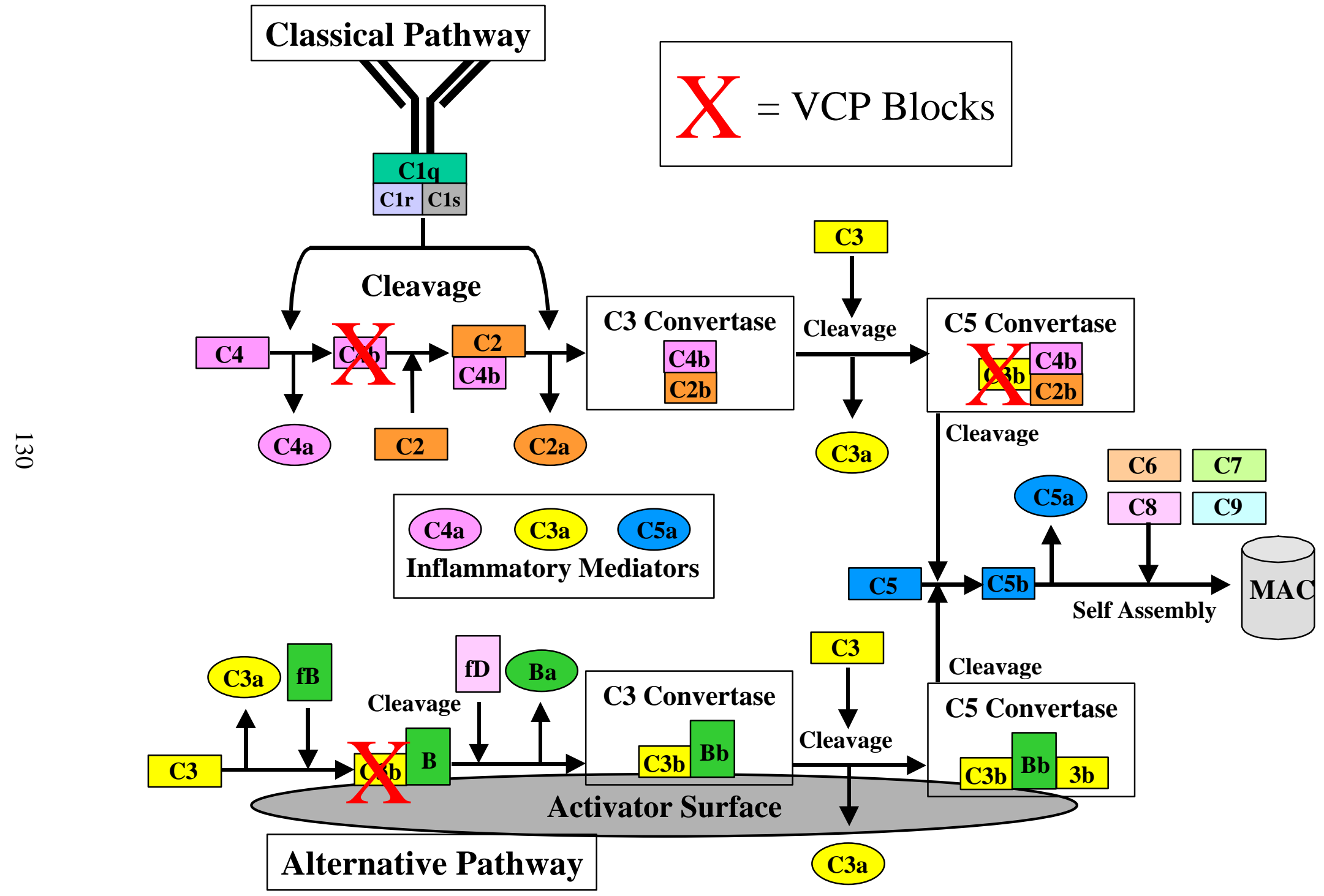


that VCP's affinity and/or activity for binding and inhibiting complement activation may be stronger for one animal species as compared to another. The animal species that vaccinia virus most commonly infects would be most susceptible to the complement inhibiting action of VCP. To initially test this hypothesis, the activity of VCP and rVCP was tested against various animal sera, including: pig, dog, rabbit, rat, human, horse, and baboon. Interestingly, VCP and rVCP possessed different activities against many of these sera.

To determine whether full-length rVCP is needed for hemolysis inhibition, factor I cofactor activity, or if one or more of the rVCP constructs retains this activity, each of the constructs purified and described in chapter 3 were tested in the hemolysis assay. In addition, V.N. Loparev, from the Poxvirus Section, Viral Exanthems and Herpesvirus Branch, Division of Viral and Rickettsial Diseases, National Center for diseases, Centers for Disease Control and Prevention, Atlanta, GA, used the hemolysis assay to test the activity of the monkeypox homologue of VCP. This VCP homologue is naturally truncated at its C-terminus, missing $75 \%$ of the fourth CCP module (figures 20 and 21).

In order to determine whether full-length rVCP is required to bind complement, various rVCP constructs were created using the Pichia pastoris expression system and subjected to plasmon resonance binding analysis (Biacore $\mathrm{X}^{\circledR}$ ) with $\mathrm{C} 3 \mathrm{~b}$. With $\mathrm{C} 3 \mathrm{~b}$ immobilized to the sensor chip, recombinant VCP or truncated rVCP molecules, consisting of CCP modules 1 and 2 (rVCP 1,2), 2 and 3 (rVCP 2,3), 3 and 4 $(\mathrm{rVCP} \sim 3,4)$, and 2-4 (rVCP 2,3,4), were injected to determine whether they possessed the ability to bind $\mathrm{C} 3 \mathrm{~b}$. In addition, with rVCP immobilized to a CM5 sensor chip, methylamine treated C3 or C4 (C3-MA, C4-MA) was injected and binding was observed. 
In all cases, positive binding was determined by subtraction of ligand channel readings from the readings of the control channel (nothing immobilized). Each experiment was repeated several times to confirm results.

The ability of rVCP to perform both of its functions simultaneously, binding complement and heparin, was tested using a modified hemolysis assay. This modified assay tests the ability of rVCP to inhibit hemolysis of sheep red cells in the presence of various concentrations of heparin or heparan sulfate. The results of these preliminary experiments suggest that $\mathrm{rVCP}$ is in fact able to bind complement while remaining bound to heparin by the C-terminus. To conclusively demonstrate this ability, Shrihari Arjunwadkar, from the Division of Medical Virology, University of Cape Town, Cape Town, South Africa, used plasmon resonance binding analysis (Biacore $\mathrm{X}^{\circledR}$ ). With heparin immobilized to the sensor chip (described in chapter 3), rVCP was injected and binding to heparin was observed. Shortly after injection of rVCP stopped, without regeneration, methylamine treated $\mathrm{C} 3$ or $\mathrm{C} 4$ (C3-MA, C4-MA) was injected to test for binding to rVCP. As a control, C3-MA and C4-MA were injected in the absence of rVCP, to test whether they possessed the ability to interact with heparin (data not shown). The results suggest that C3-MA and C4-MA do interact with heparin, but only slightly. The interaction was very small in comparison to that seen in the presence of rVCP. Each experiment was repeated three times to confirm the results and positive binding was observed by channel subtraction.

Using the results obtained by the above experiments, results obtained in chapter 3 , and homology found between rVCP and other complement control proteins, a model of the putative heparin and/or complement binding residues was created. These putative 
binding domains were visualized using the X-ray crystal structure of rVCP. The residues involved in complement binding were predicted using rVCP's homology with the wellcharacterized MCP. A recent study subjected MCP to extensive alanine-scanning mutagenesis and epitope mapping, which revealed the residues involved in cofactor activity and involved in binding C3b and/or C4b (Liszewski et al., 2000). The homology between VCP and MCP is so great that these residues could very confidently be projected upon VCP and thought to contribute the same function. 


\section{$\underline{\text { Materials and Methods }}$}

\section{Hemolysis Assay:}

The hemolysis assay was performed as described in chapter 2 with a few modifications. Serial dilutions of rVCP were mixed with various amounts of heparan sulfate (HS) (Sigma) before addition of serum and sSRBCs. In assays using heparin sepharose, $10 \mu \mathrm{l}$ of slurry, made from $1 \mathrm{ml}$ heparin sepharose (Pharmacia) and $1 \mathrm{ml}$ water, were added to a series of rVCP dilutions and incubated for $30 \mathrm{~min}$ on ice. After the incubation, the samples were centrifuged and the supernatants tested for hemolysis activity. The heparin sepharose beads were then washed with buffer before serum and sensitized sheep red blood cells were added. After the assay was performed, reaction supernatants and the pelleted heparin sepharose beads were analyzed by SDS-PAGE for the presence of rVCP. Supernatants were concentrated and the heparin beads resuspended in $600 \mathrm{mM} \mathrm{NaCl}$, to strip off rVCP, and concentrated. In all cases, both positive and negative controls received the same amount of heparin/heparan as all of the samples, in order to compensate for lysis inhibition caused by the heparin/heparan.

\section{Preparation of $r V C P$ and $r V C P$ Truncations:}

The cloning of $\mathrm{VCP}$ and fragments $\mathrm{rVCP} \sim 1,2 ; \mathrm{rVCP} \sim 2,3$; rVCP 3,4; rVCP 2,3,4 in the Pichia pastoris yeast expression system (Invitrogen) was described in chapter 2. rVCP; rVCP 1,2; rVCP 3,4; rVCP 2,3,4 were all purified from media using heparin (Pharmacia) affinity chromatography, described in chapter 2. $\mathrm{rVCP} 2,3$ was purified by ion exchange chromatography using a $1 \mathrm{ml}$ DEAE HiTrap fast flow (Pharmacia) column. All proteins were visualized by SDS-PAGE (4-12\% gel) with coomassie blue staining. 


\section{Complement Component Purification:}

Initial purification of $\mathrm{C} 3$ and $\mathrm{C} 4$ was performed by Gunasekaran Krishnasamy, from the Center for Biophysical Science and Engineering, University of Alabama at Birmingham, AL. Three milliliter of inhibitor solution containing $330 \mathrm{mM} \mathrm{KH} \mathrm{PO}_{4}(\mathrm{pH}$ 7.3), $66 \mathrm{mM}$ EDTA, $66 \mathrm{mM}$ benzamidine- $\mathrm{HCl}$ and $1 \mathrm{~g}$ of Lysine (free base) was added to the $20 \mathrm{ml}$ of blood plasma. The resultant treated plasma was added to polyethylene glycol 4000 D (PEG4K) for precipitation. The final concentration of PEG in the solution was $5 \%$. After removing the precipitate, the plasma solution was further made $21 \%$ PEG4K. The solution was stirred in cold room at $4^{\circ} \mathrm{C}$ for about $2 \mathrm{hrs}$. The precipitate formed was dissolved in $7 \mathrm{ml}$ of DEAE starting buffer [3 mM KH $2 \mathrm{PO}_{4}(\mathrm{pH} 7.3), 6.5 \mathrm{mM}$ EDTA, $6.5 \mathrm{mM}$ benzamidine- $\mathrm{HCl}, 1 \mathrm{mM}$ phenylmethylsulfonyl fluoride (PMSF), $40 \mathrm{mM}$ EACA and $30 \mathrm{mM} \mathrm{NaCl}]$. The sample was applied on a $100 \mathrm{ml}$ DEAE column, and after washing with two column volumes of starting buffer, a gradient was developed from 30 $\mathrm{mM}$ to $150 \mathrm{mM} \mathrm{NaCl}$. Those fractions containing $\mathrm{C} 3$ were dialyzed against a buffer containing $25 \mathrm{mM}$ Tris (pH 7.8), $50 \mathrm{mM}$ EACA, 2 mM EDTA, $2 \mathrm{mM}$ benzamidine- $\mathrm{HCl}$ and $50 \mathrm{mM} \mathrm{NaCl}$. The dialyzed sample was applied on a $5 \mathrm{ml}$ Blue Sepharose column and eluted with a gradient of 50-500 mM NaCl (Gresham et al., 1986).

To produce C3b, purified C3 was further purified by perfusion chromatography using BioCAD 20HQ column - see figure 34. C3b was prepared by limited trypsin digestion of $\mathrm{C} 3$ with sequencing grade trypsin (Roche). C3 was incubated with 5\% $\operatorname{trypsin}(\mathrm{w} / \mathrm{w})$ for $2 \mathrm{~min}$ at $37^{\circ} \mathrm{C}$, before adding $5 \%(\mathrm{w} / \mathrm{w})$ soybean trypsin inhibitor (SBTI) (Sigma) to stop the reaction. C3 and C3b were visualized by SDS-PAGE (4-12\% gel) with coomassie blue staining. 
Figure 34. C3 Purification using BioCAD 20HQ Column. Purification of C3 was achieved using BioCAD 20HQ ion exchange chromatography. The top panel shows the first passage, with a large amount of unbound material. The absorbance is shown in red and the $\mathrm{NaCl}$ gradient shown in blue. As can be seen in this panel, the $\mathrm{C} 3$ peak appeared half way through the $\mathrm{NaCl}$ gradient (SDS-PAGE analysis of this peak is shown in figure 39), indicating strong binding. The bottom panel shows a rerun of the unbinding material from the top panel, showing that all of the initial unbound material passed through the column without binding a second time. 


\section{C3 Purification using BioCAD 20HQ Column}
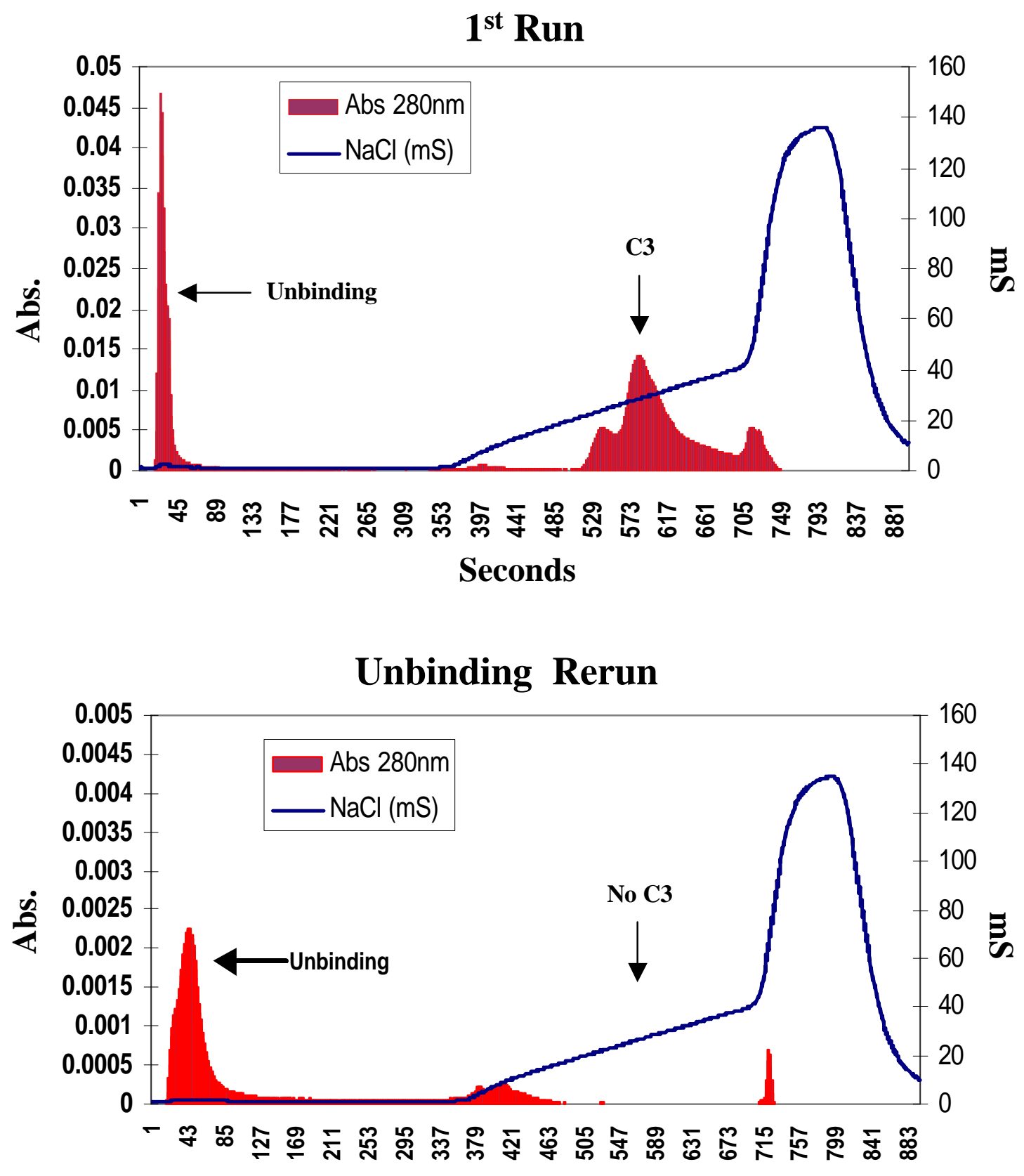

Seconds 
To isolate C4, $5 \mathrm{mM}$ PMSF, $5 \mathrm{mM}$ EDTA and $2.7 \mathrm{mM}$ iodoacetamide were added to $20 \mathrm{ml}$ of plasma and the $\mathrm{pH}$ was adjusted to 7.4. After 5\% PEG precipitation, the resultant supernatant was made 26\% PEG. The precipitate obtained was dissolved and diluted to a conductivity of $2.9 \mathrm{mS}$ in $20 \mathrm{mM}$ phosphate buffer (pH 7.0) with $5 \mathrm{mM}$ EDTA, $5 \mathrm{mM}$ PMSF and $10 \mathrm{mM} \mathrm{NaCl}$. The sample was applied to a DEAE-cellulose (DE-52 cellulose) column and eluted with the same buffer. The eluted fractions containing C4 were applied to a DE-52 cellulose column a second time and eluted with a gradient of 30-200 mM NaCl (Reboul et al., 1979; Press and Gagnon, 1981).

The purified $\mathrm{C} 3$ and $\mathrm{C} 4$ were dialyzed against $20 \mathrm{mM} \mathrm{KH}_{2} \mathrm{PO}_{4}, 2$ mM EDTA, $100 \mathrm{mM} \mathrm{NaCl}, 20 \mathrm{mM}$ EACA (pH 7.5). The C3 or C4 was treated with $20 \mathrm{mM}, 50 \mathrm{mM}$ and $100 \mathrm{mM}$ methylamine in steps for a period of $3 \mathrm{hrs}$ at $4^{\circ} \mathrm{C}$ (Tack et al., 1980). Then, the solution was allowed to stand in the same temperature for about $10 \mathrm{hrs}$. The resultant methylamine-treated $\mathrm{C} 3$ or $\mathrm{C} 4$ was dialyzed against $20 \mathrm{mM} \mathrm{KH} \mathrm{KO}_{4}, 2 \mathrm{mM}$ EDTA and $100 \mathrm{mM} \mathrm{NaCl}(\mathrm{pH} 7.5)$ and concentrated.

\section{Primary and Secondary Antibody Binding Analysis:}

The binding between a mono-specific anti-rVCP $\operatorname{IgY} 1^{\circ}$ antibody (Washington Biotec, described in chapter 2) and rVCP was visualized using BiaEvaluation 3.0 software system. With rVCP amine coupled to a CM5 sensor chip, $40 \mu \mathrm{l}$ of $10 \mathrm{nM}$ purified mono-specific $\operatorname{IgY}$ was diluted in HBS, and injected with a flow rate of 10 $\mu \mathrm{l} / \mathrm{min}$ at $25^{\circ} \mathrm{C}$. The wash was delayed for 600 seconds. The binding between a $2^{\circ}$ antichicken IgG (Vector Laboratories) and the $1^{\circ}$ mono-specific anti-rVCP IgY was 
visualized by the same method. The $1^{\circ}$ antibody was immobilized and the $2^{\circ}$ antibody was used as the analyte.

\section{Simultaneous Heparin and Complement Binding:}

Hemolysis assays were performed as described in chapter 2, with a few modifications. Serial dilutions of rVCP were mixed with various amounts of heparan sulfate (HS) (Sigma) before addition of serum and sensitized sheep red blood cells. In assays using heparin sepharose, $10 \mu \mathrm{l}$ of slurry, made from $1 \mathrm{ml}$ heparin sepharose (Pharmacia) and $1 \mathrm{ml}$ water, was added to a series of rVCP dilutions and incubated for 30 min on ice. After the incubation, the samples were centrifuged and the supernatants tested for hemolysis activity. The heparin sepharose beads were then washed with buffer before serum and sSRBCs were added. After the assay was performed, reaction supernatants and the pelleted heparin sepharose beads were analyzed by SDS-PAGE for the presence of rVCP. Supernatants were concentrated and the heparin beads resuspended in $600 \mathrm{mM} \mathrm{NaCl}$, to strip off rVCP, and concentrated. In all cases, both positive and negative controls received the same amount of heparin/heparan as all of the samples, in order to compensate for lysis inhibition caused by the heparin/heparan.

\section{X-Ray Crystallography:}

The procedures for the X-ray crystallography are described in chapter 3 . Homology with MCP was obtained using a BLAST search and through comparison with the study by Liszewski et al., 2000. 


\section{$\underline{\text { Results }}$}

\section{Hemolysis Assay of Various Animal Sera:}

The ability to block the activity of complement in various animal species was tested using the hemolysis assay. The outcomes of experiments using VCP and rVCP are presented in figure 35 . VCP was found to behave very similarly to rVCP with most animal sera tested - see figure 36 . For rat sera, the results very strongly suggest that VCP has the greatest degree of activity against rat sera. Melanie Scott, from the Department of Microbiology and Immunology, University of Louisville, obtained very similar results using mouse sera (personal communication). In addition, VCP clearly possesses very limited activity against rabbit sera. Most importantly, however, is the moderate activity that VCP exhibits against human sera. This activity was also found to be identical in baboon (data not shown). Therefore, VCP was found to have very strong activity against rat, moderate activity against human, and little activity against rabbit sera.

\section{Hemolysis Assay of rVCP Constructs:}

The activity of the purified recombinant protein constructs was tested using the hemolysis assay. The results indicate that only the full-length protein inhibits lysis of sSRBCs - anywhere from 60 to $90 \%$ inhibition. The rVCP segments showed no inhibition of lysis, suggesting that the whole protein is needed to block complement activation. Although, the naturally truncated VCP homologue produced by monkeypox virus (MPV) (shown in figure 37), which lacks almost the entire fourth SCR (figures 20 and 21), has been shown to inhibit hemolysis of sSRBCs. This indicates that it is blocking the classical pathway of complement activation. 
Figure 35. Hemolysis assay of various animal sera. The ability of VCP and rVCP to block the activation of complement in various animal sera was tested. A) Silver-stained SDS-PAGE gel showing the concentration of $\mathrm{VCP}$ and $\mathrm{rVCP}$ used in the experiment. B) The ability of VCP, produced by the natural infection of mammalian cells, to inhibit the activation of complement in dog, horse, human, pig, rabbit, and rat sera was tested. The results clearly indicate that VCP possesses the greatest activity against rat sera (and pig) and the lowest activity against rabbit sera. C) The ability of rVCP, produced by the Pichia pastoris yeast expression system, to inhibit the activation of complement in dog, horse, human, pig, rabbit, and rat sera was tested. Again, the results clearly indicate that rVCP possesses the greatest activity against rat sera and the lowest activity against rabbit sera. 

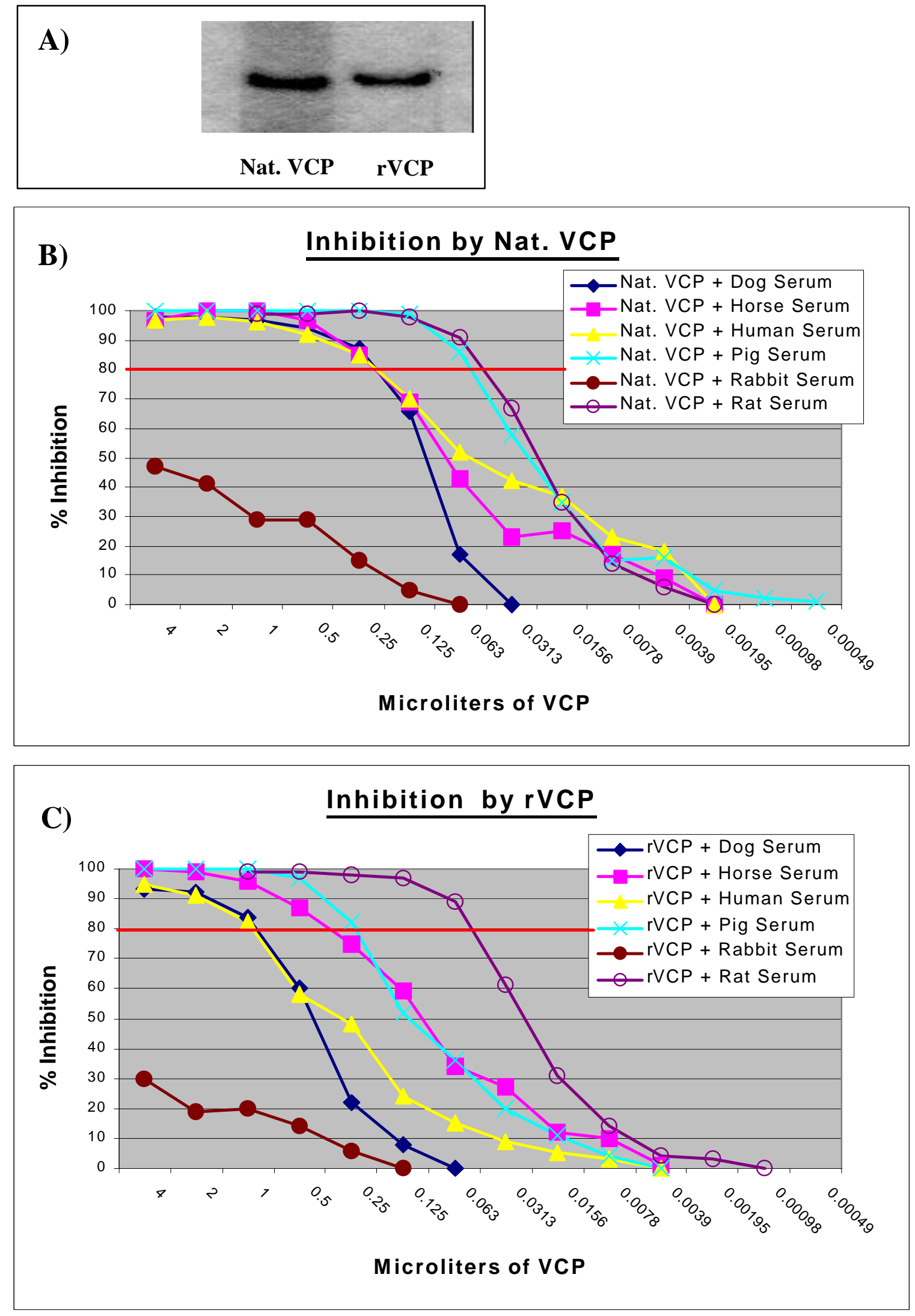
Figure 36. Hemolysis assay of rat sera using VCP and rVCP. The activity of VCP and rVCP against complement activation using rat sera is shown - data taken from figure 35. The concentration of VCP and rVCP were estimated to be the same and the activity exhibited against rat serum complement is shown to also be the same. This demonstrates that the activity of VCP and rVCP are identical against rat sera. 


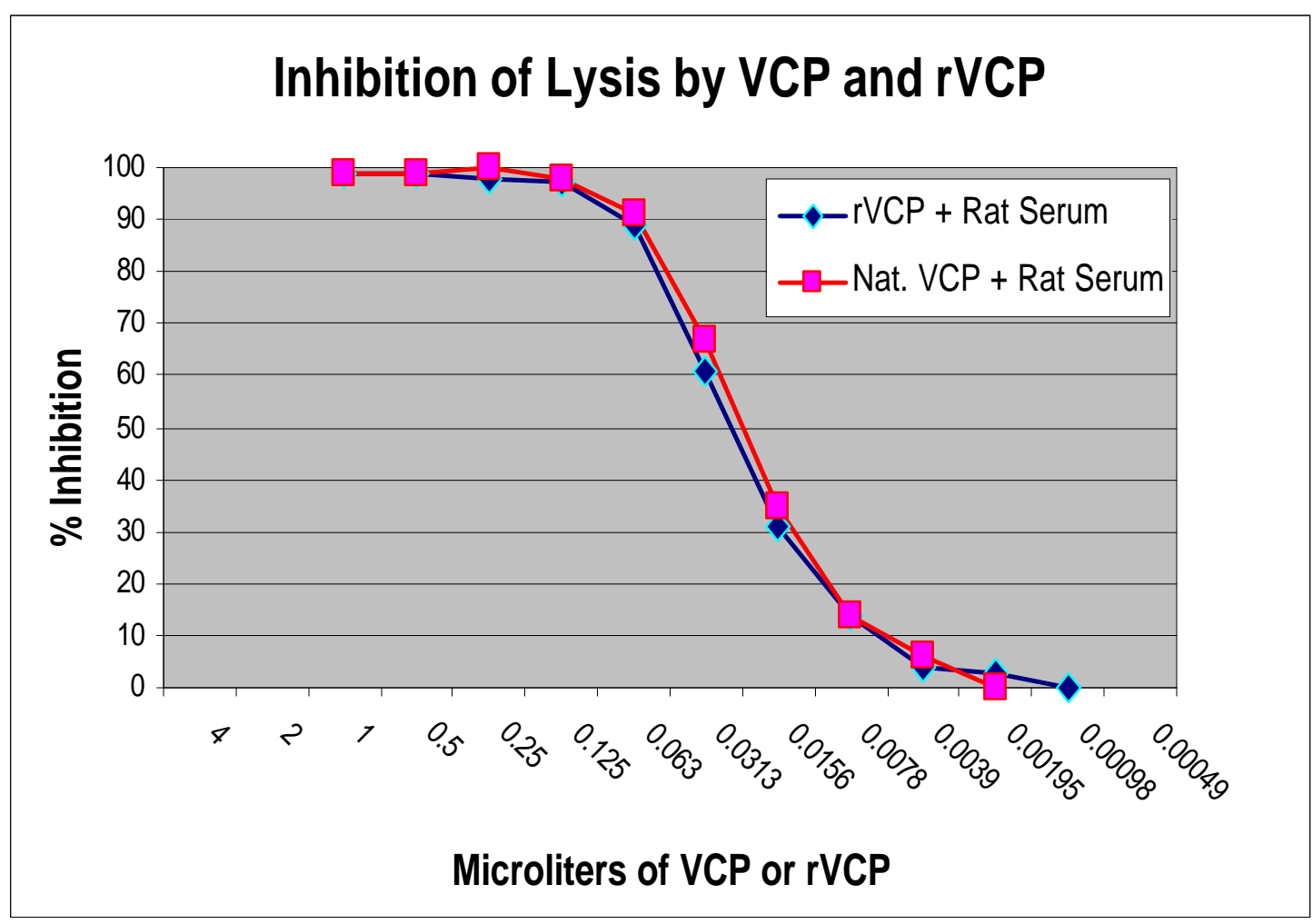


Figure 37. Structure-function summary table of VCP, VCP homologues, and rVCPs. VCP/IMP/SPICE, MPV homologue of VCP, recombinant VCP, and four recombinant segments of $\mathrm{VCP}$ are shown along with whether they are able to inhibit hemolysis of sSRBCs, bind C3b using SPR, or bind heparin using chromatography or SPR. Also listed is the number of putative heparin binding sites found on the surface of the protein. 


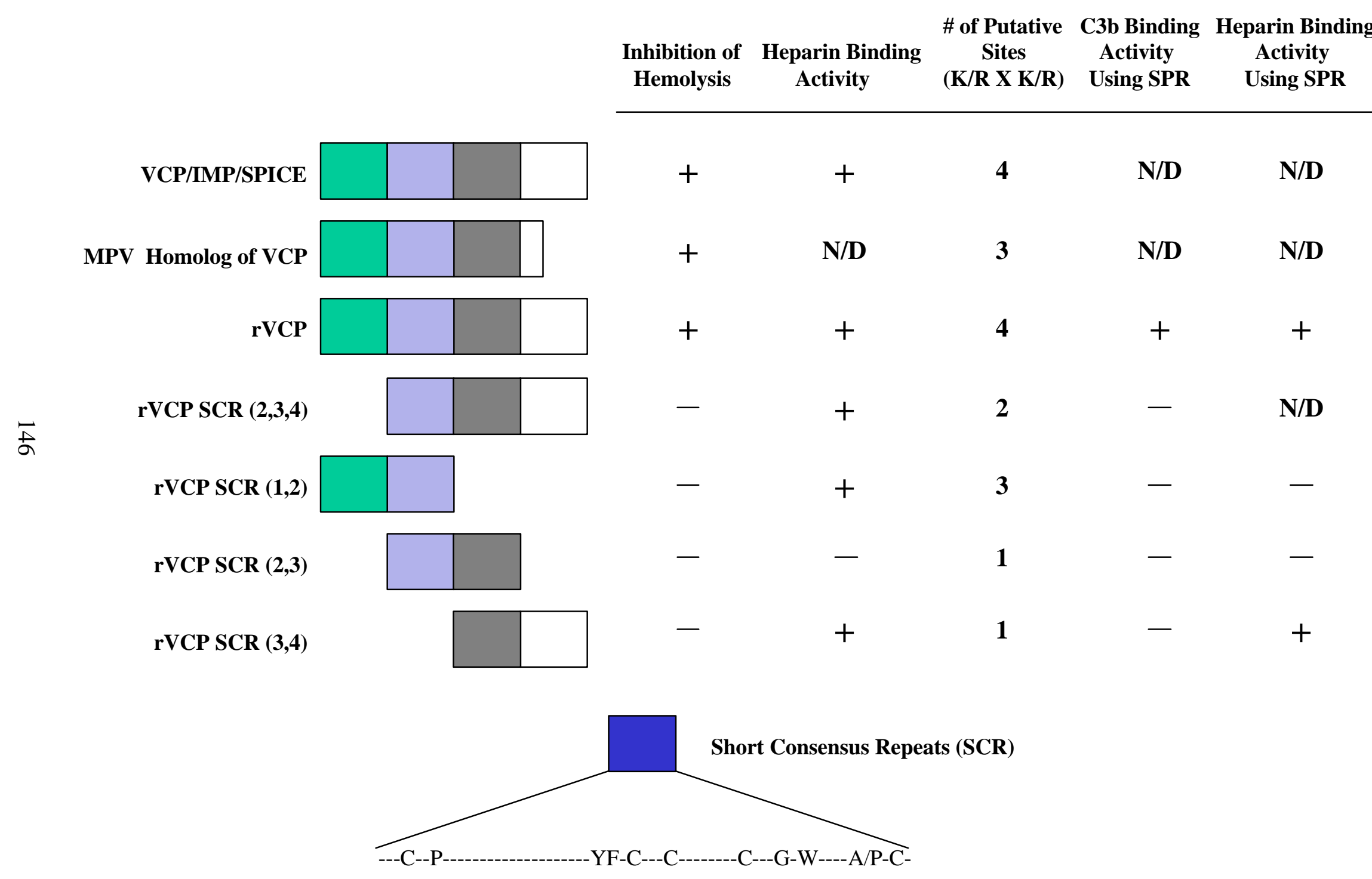




\section{Biacore Complement Binding:}

As a control to determine whether the Biacore instrument was functioning properly, rVCP was immobilized on a CM5 sensor chip by standard amine coupling. 10 $\mathrm{nM}$ of purified mono-specific chicken anti-rVCP IgY, primary antibody, was injected across the surface of the sensor chip and very strong binding was observed, see figure 38A. In addition, this primary anti-rVCP antibody was immobilized to a CM5 sensor chip by standard amine coupling procedures. $10 \mathrm{nM}$ of biotinylated goat anti-chicken IgG (Vector Laboratories), secondary antibody, was passed across the immobilized antiVCP antibody and extremely strong binding was observed, see figure 38B.

To better understand the binding of $\mathrm{rVCP}$ to $\mathrm{C} 3 \mathrm{~b}, \mathrm{C} 3$ was subjected to incomplete trypsin digestion, followed by amine coupling to a CM5 sensor chip. Using recombinantly expressed rVCP and fragments of rVCP, the location for $\mathrm{C} 3 \mathrm{~b}$ binding on rVCP was mapped - see figure 39 for the protein reagents. Interestingly, none of the recombinant proteins, representing nearly all combinations of two and three CCP modules found in rVCP, had the ability to bind. As can be seen in figure $40, \mathrm{rVCP}$ is the only protein found to bind $\mathrm{C} 3 \mathrm{~b}$ immobilized to the sensor chip. By comparing the ligand channel response to the control channel response, it is very clear that all segments of $\mathrm{rVCP}$ are unable to bind the immobilized $\mathrm{C} 3 \mathrm{~b}$. In all cases, the analyte was injected at different concentrations to confirm the results. The amplitude of binding was found to be low due to the incomplete cleavage of $\mathrm{C} 3$ to $\mathrm{C} 3 \mathrm{~b}$, resulting in less immobilized $\mathrm{C} 3 \mathrm{~b}$ on the surface of the sensor chip. In addition, the best line fitting was obtained using the heterologous ligand model, due to a mixed population of C3 and C3b (data not shown). This data suggests that rVCP has a very weak ability to interact with native $\mathrm{C} 3$ and a 
Figure 38. Control $\mathbf{1}^{\circ}$ and $2^{\circ}$ antibody binding. A) Analysis of anti-VCP $\operatorname{IgY}\left(1^{\circ}\right.$ antibody) binding to VCP. Subtracted ligand channel and control channel is shown. B) Analysis of anti-chicken $\operatorname{IgG}\left(2^{\circ}\right.$ antibody) binding to anti-VCP $\operatorname{IgY} 1^{\circ}$ antibody. Subtracted ligand channel and control channel is shown. 


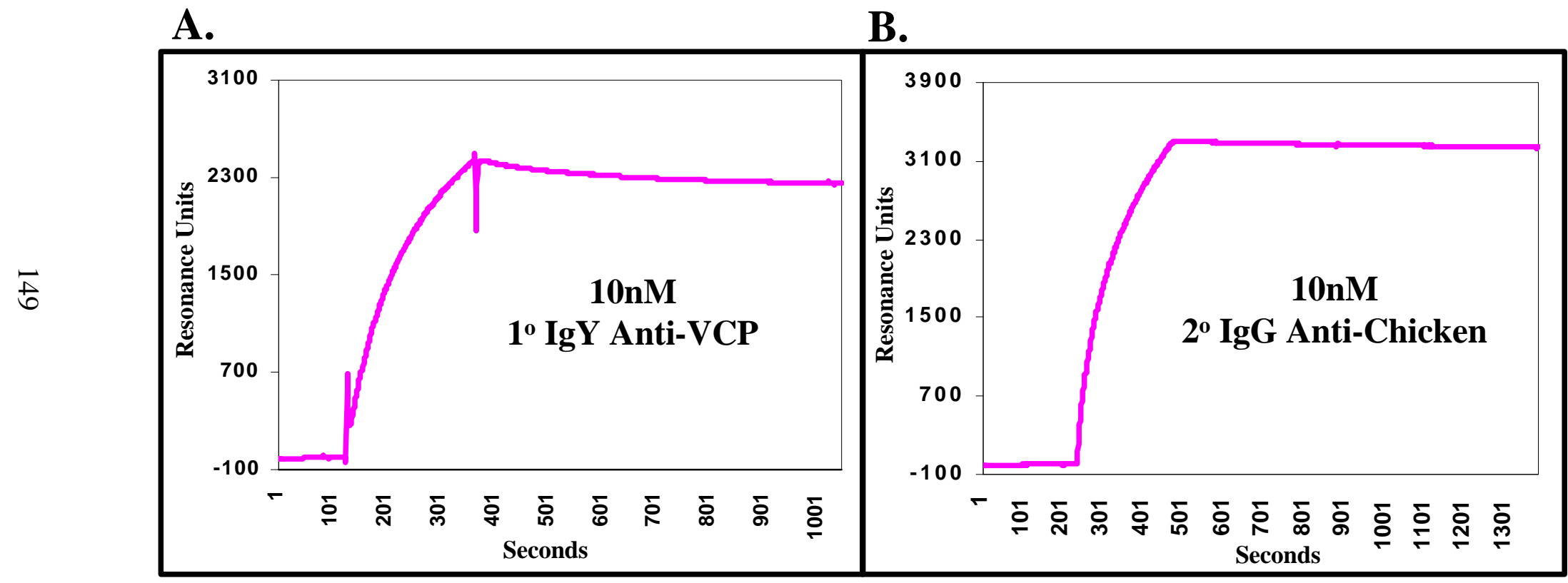


Figure 39. Coomassie blue stained SDS-PAGE of C3, C3b, and rVCP constructs. A) (1) Purified $\mathrm{C} 3 \alpha$ and $\beta$ chains before trypsinization. (2) $\mathrm{C} 3 \mathrm{~b}$ after trypsinization. Note that incomplete trypsinization resulted in approximately $80 \%$ of the $\mathrm{C} 3$ being converted into $\mathrm{C} 3 \mathrm{~b}$, leading to a mixed ligand. B) Purified $\mathrm{rVCP}$ and $\mathrm{rVCP}$ fragments (approximately $20 \mu \mathrm{g}$ ) are shown as: (1) $\mathrm{rVCP}$; (2) $\mathrm{rVCP} 1$,2; (3) $\operatorname{rVCP} 2,3$; (4) rVCP 3,4; and (5) rVCP 2,3,4. 


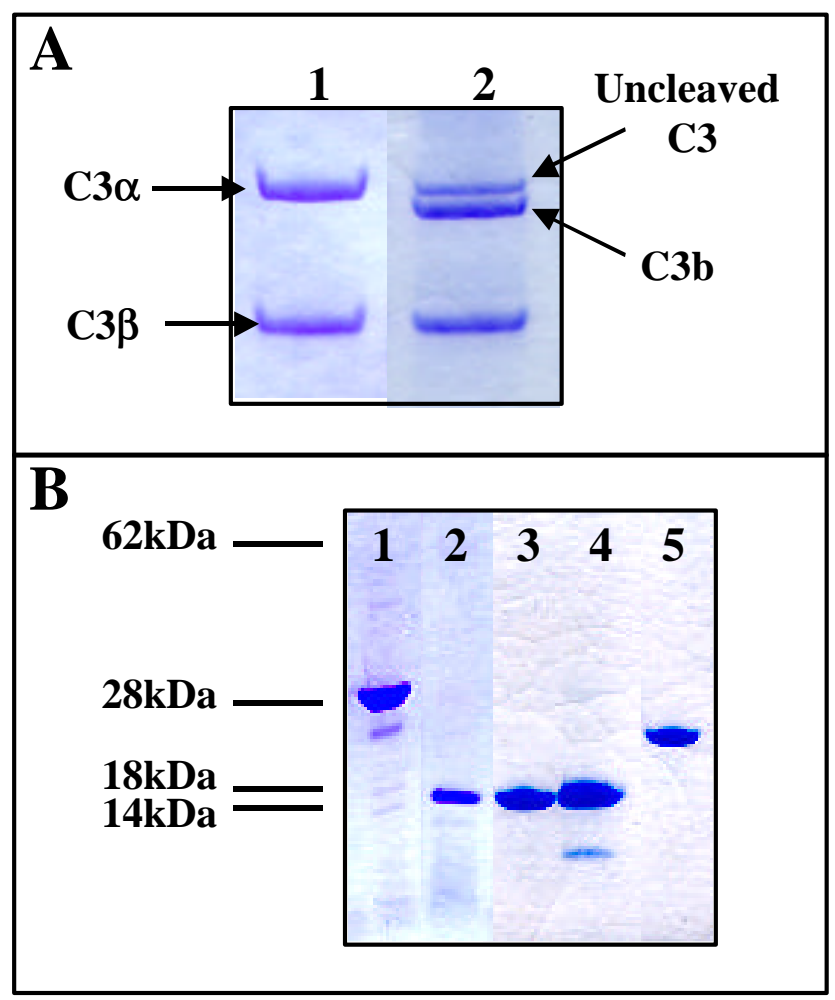


Figure 40. C3b binding of rVCP fragments. The ligand and control channel responses are shown for the binding between $\mathrm{rVCP}$, or $\mathrm{rVCP}$ fragments, and $\mathrm{C} 3 \mathrm{~b}$ immobilized to the sensor chip. Control channel response represents only bulk effects; no protein is immobilized. All proteins were tested at different concentrations and with multiple C3b coupled chips to confirm the presented results. 


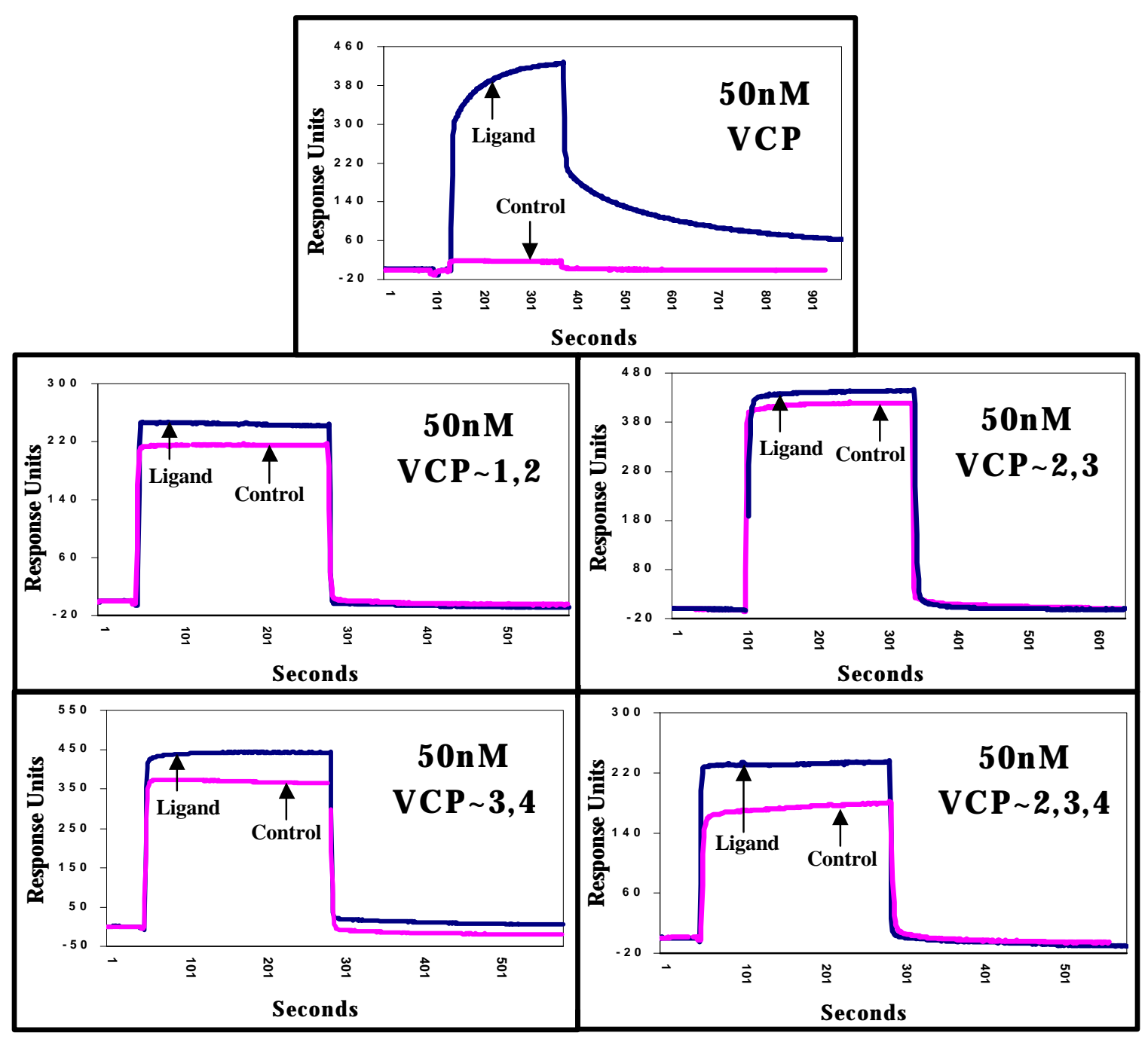


much stronger ability to interact with $\mathrm{C} 3 \mathrm{~b}$. This ability of rVCP to bind, at least weakly, to C3 is in agreement with data obtained earlier (Sahu et al., 1998). In addition, the ability of rVCP to bind methylamine treated C3 or C4 (C3-MA, C4-MA) was tested. With rVCP immobilized to the sensor chip, 80 nM C3-MA or 80 nM C4-MA was injected and binding was recorded. As can be seen in figure 41, rVCP binds strongly to both C3-MA and C4-MA. This demonstrates the ability of rVCP to bind the activated forms of the third and fourth components of the complement system. Taken together, these results suggest that VCP is the smallest unit required for binding $\mathrm{C} 3 \mathrm{~b}$ and facilitating factor I cofactor function, thus blocking activation of complement.

\section{Simultaneous Heparin and Complement Binding:}

Since rVCP is an extended molecule, and given the probable location of a heparin binding site at the extreme $\mathrm{C}$-terminal end and putative regions involved in binding complement spread throughout the remainder of this extended molecule, it might be expected that $\mathrm{C} 3 \mathrm{~b} / \mathrm{C} 4 \mathrm{~b}$ interactions and heparin binding are not mutually exclusive. Participation of common residues in heparin binding and complement inhibition in rVCP is not supported by the observation that rVCP is able to inhibit hemolysis of sSRBCs and bind heparin simultaneously (figure 42 and 43). Addition of varying amounts of soluble heparin (figure 42) or heparan sulfate to a hemolysis assay containing rVCP had no effect on the protein's ability to inhibit hemolysis (figure 43a). The hemolysis inhibition seen in the cases when only heparin or heparan sulfate is present in high concentrations is likely due to its slight ability to bind $\mathrm{C} 3 / \mathrm{C} 4$, thus inhibiting hemolysis on its own. A similar experiment employing heparin sepharose beads, used to carry rVCP into the 
Figure 41. rVCP binding to activated $\mathbf{C 3}$ and C4. rVCP was immobilized to a CM5 sensor chip. A) $80 \mathrm{nM} \mathrm{C3-MA} \mathrm{was} \mathrm{injected} \mathrm{and} \mathrm{binding} \mathrm{was} \mathrm{observed.} \mathrm{B)} 80 \mathrm{nM} \mathrm{C4-}$ MA was injected and binding was observed. In both cases, the results are shown as the subtraction of the control channel from the ligand channel. 
A. Interaction of methyl-C3 with VCP immobilized on CM5 chip

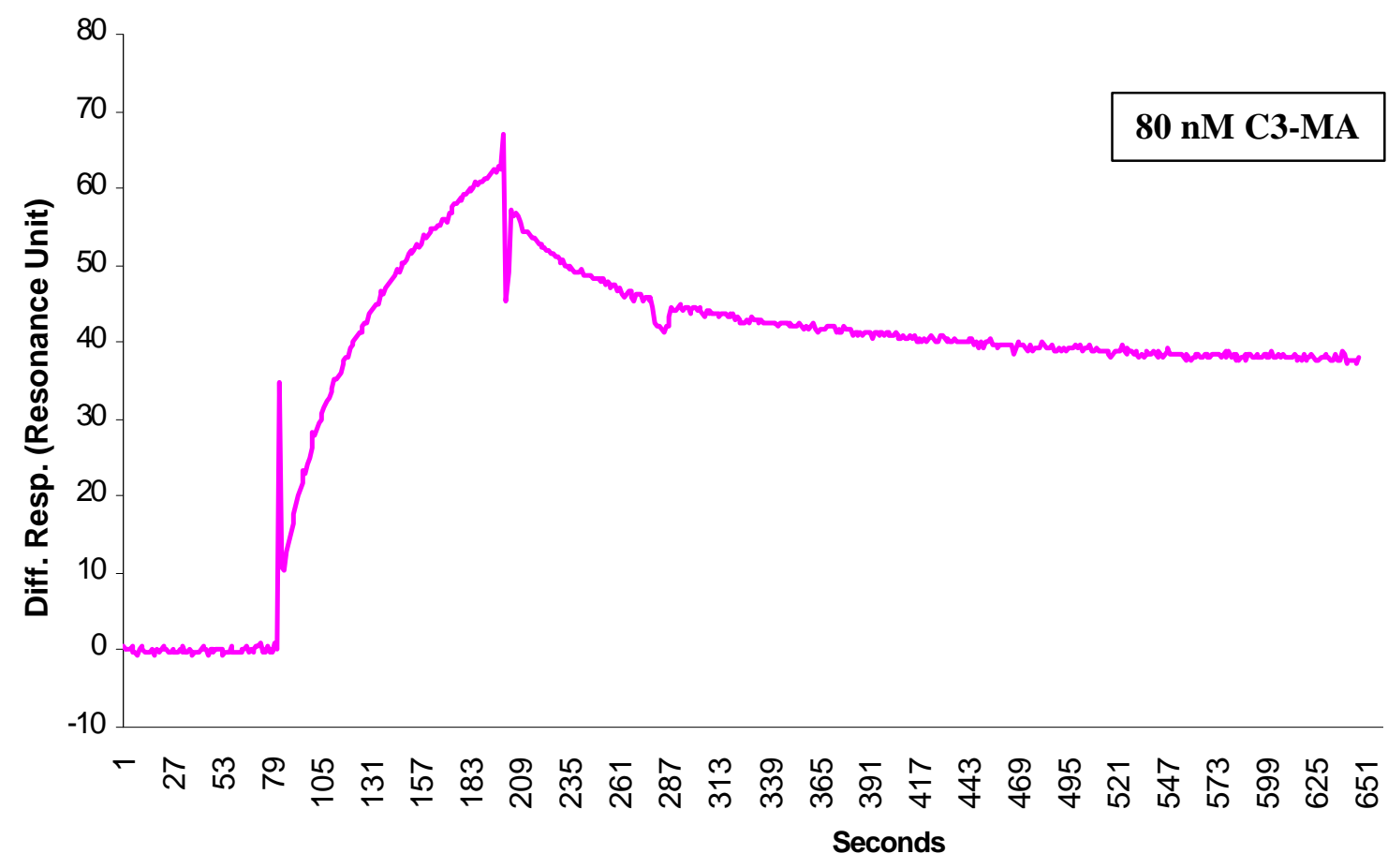

B.

Interaction of methyl-C4 with VCP immobilized on CM5 chip

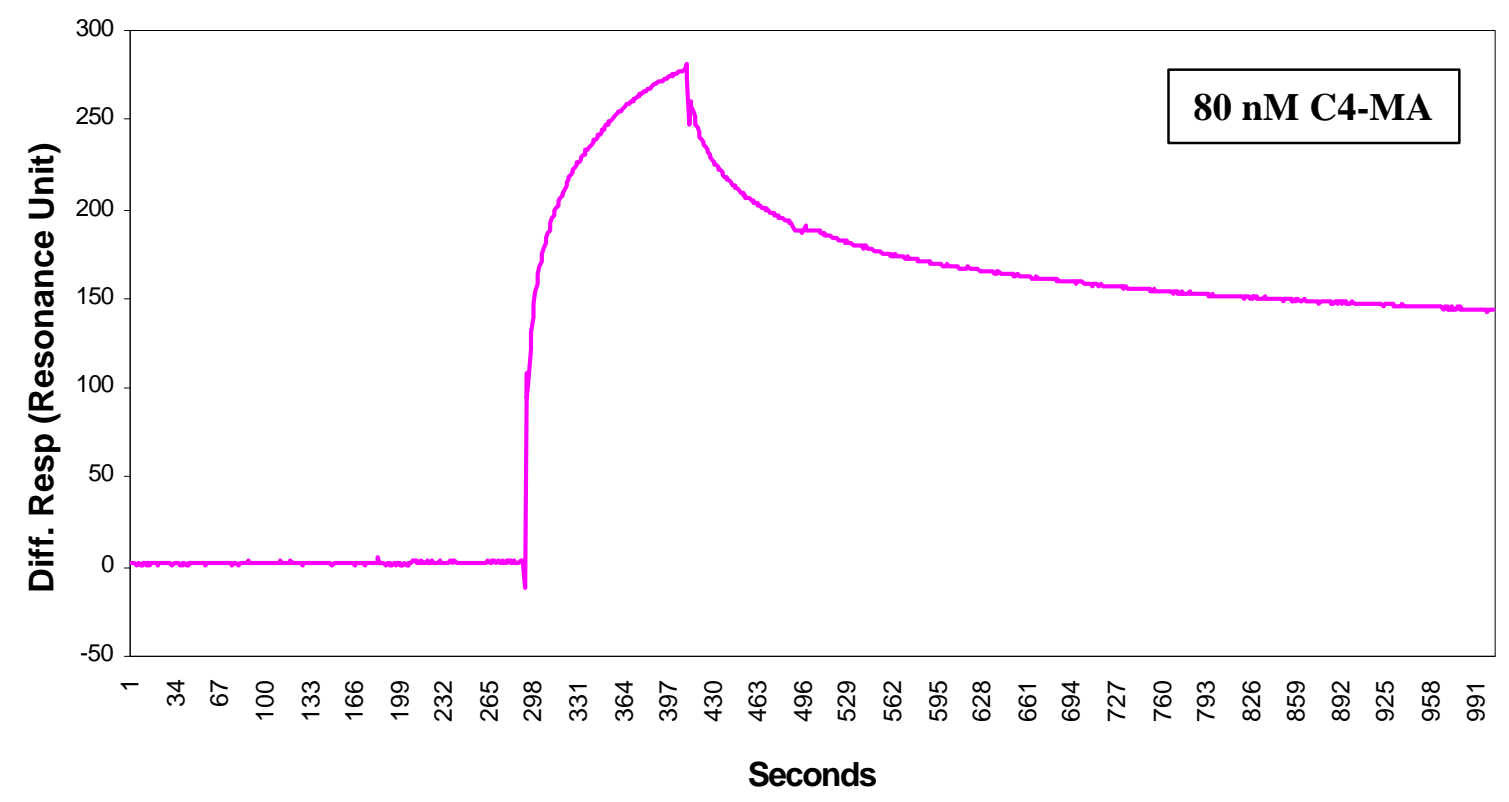


Figure 42. Simultaneous heparin binding and hemolysis inhibition by rVCP. Results of hemolysis assays performed on serially diluted samples of rVCP each containing 0.01 , 0.03, 0.05 Units of soluble heparin (filled squares, filled triangles and open circles, respectively) are compared to the sample without heparin (control, shown as filled diamonds). The degree of hemolysis inhibition caused by heparin alone is shown as asterisks. 


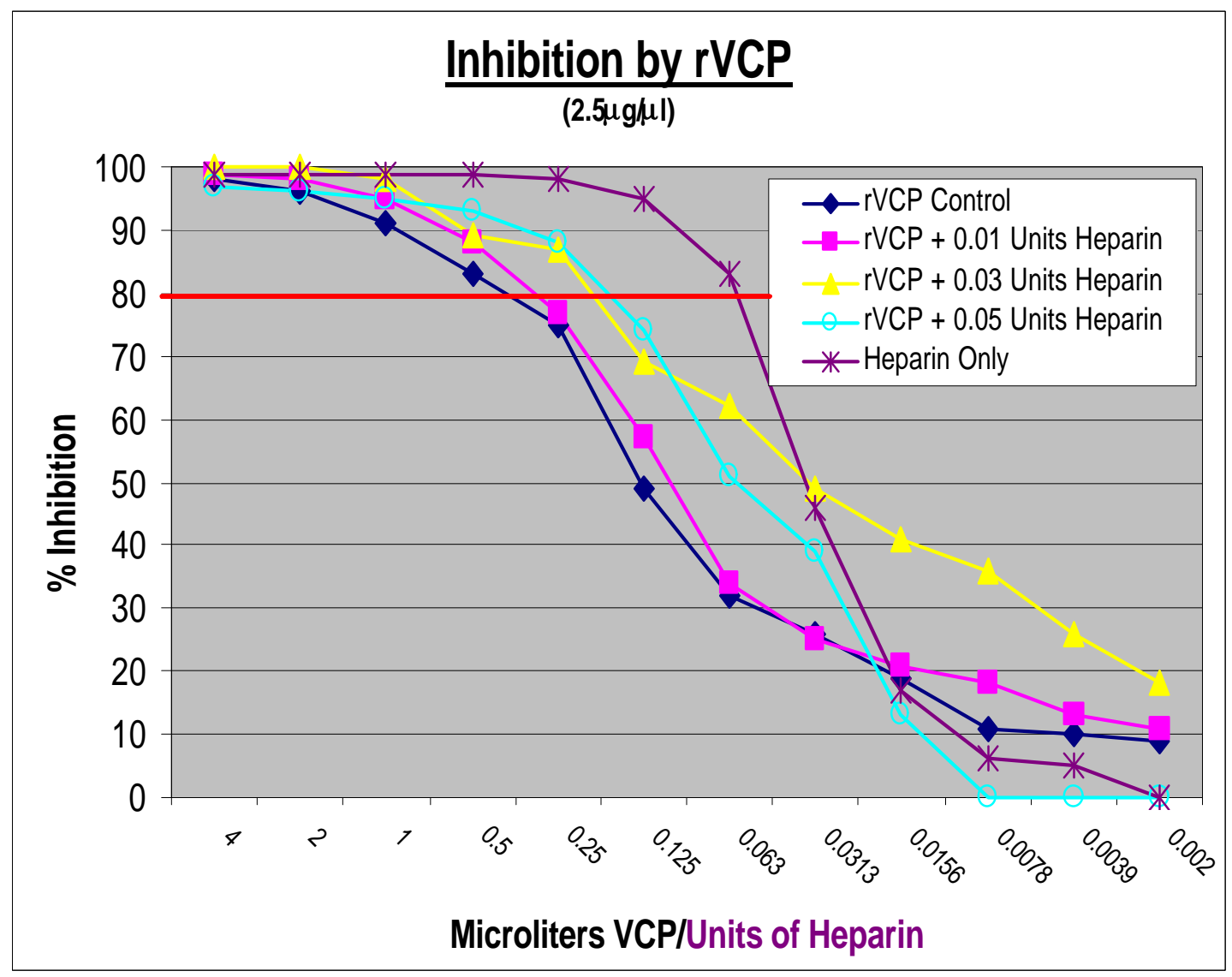


Figure 43. Simultaneous heparan sulfate binding and hemolysis inhibition by rVCP. a) Results of hemolysis assays performed on serially diluted samples of rVCP each containing $0.01,0.1,1.0 \mu \mathrm{g}$ of soluble heparan sulfate with an average molecular weight of 7,500 (filled circles, open triangles and open circles, respectively) are compared to the sample without heparan sulfate (control, shown as filled squares). The degree of hemolysis inhibition caused by heparan sulfate alone is shown as asterisks. b) The results of a hemolysis assay performed on rVCP bound to heparin sepharose beads are compared to the sample without heparin sepharose (control shown as squares). The results of a hemolysis assay performed on material that did not bind to the heparin sepharose beads (supernatant) are shown as triangles. c) Coomassie blue stained SDS-

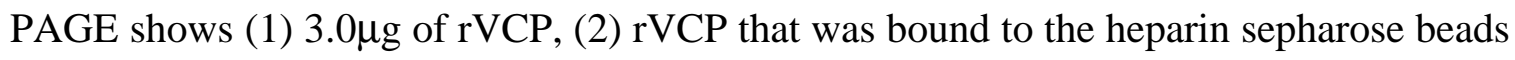
after the hemolysis assay shown above, and (3) rVCP that was contained within the supernatants after the hemolysis assay shown above. The material in lane 3 probably represents the fraction of rVCP that was incompetent in binding due to misfolding. 
a

b
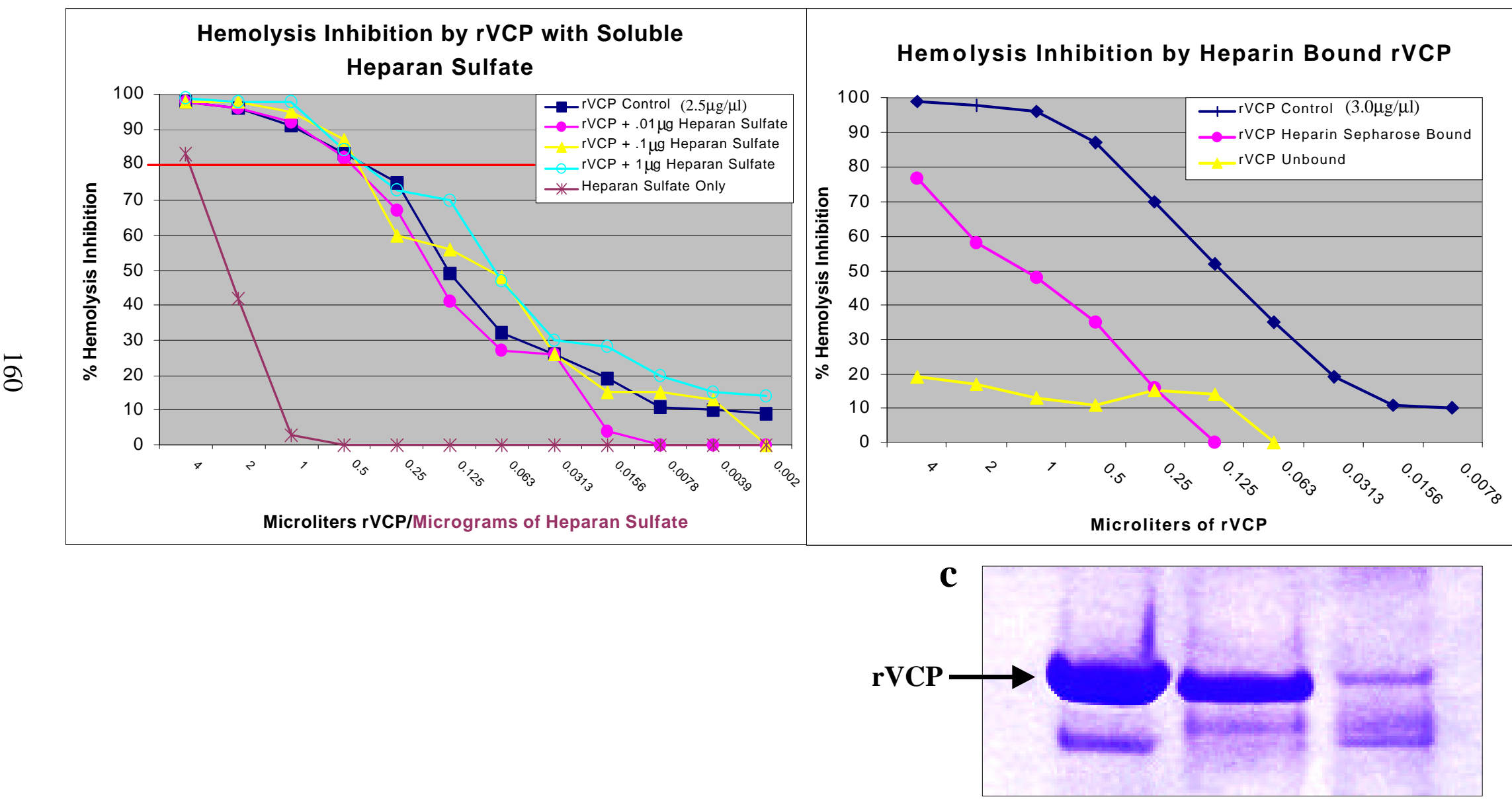

(1) (2)

(3) 
hemolysis assay, revealed that rVCP can still inhibit complement activation while remaining bound to insoluble heparin, although to a much lesser extent (figure 43b). The reaction mixture was further examined after the assay was performed in order to determine whether rVCP inhibits hemolysis while bound to heparin, or is pulled off by C3 and C4 during the reaction. Figure 43c clearly demonstrates that the hemolysisinhibiting activity could be attributed to rVCP that remained bound to the heparin sepharose beads and was not detached from the beads in the process.

To confirm the above results using SPR technology, heparin was immobilized to a CM5 sensor chip, $80 \mathrm{nM}$ of rVCP was injected, and binding was observed. Shortly after the injection of rVCP stopped, $100 \mathrm{nM}$ of C3-MA or C4-MA was injected to test if binding to rVCP could occur. As can be seen in figure 44, rVCP is able to bind complement while remaining bound to heparin immobilized to the sensor chip. These results demonstrate the ability of rVCP to perform both functions at once. With both $\mathrm{C} 3$ MA and C4-MA, strong binding was observed, indicating that rVCP is able to simultaneously bind both heparin, via its C-terminus, and complement, using the rest of its exposed surface. Using the data obtained in the above experiments, along with sequence homology data described below, a model for heparin binding and complement component binding was developed and shown in figure 45 .

\section{X-Ray Crystal Structure Homology:}

Putative sites on $\mathrm{rVCP}$, which are suspected to interact with $\mathrm{C} 3 \mathrm{~b} / \mathrm{C} 4 \mathrm{~b}$, are suggested by comparison with other complement control proteins for which extensive mutagenesis data are available. A recent study employing synthetic peptides, 
Figure 44. Simultaneous binding to heparin and complement by rVCP. A) Analysis of rVCP binding to heparin, followed by binding of methylated $\mathrm{C} 3$ to rVCP. Results are represented by subtraction of the ligand channel and the control channel. The experiment was repeated to confirm results. B) Analysis of rVCP binding to heparin, followed by binding of methylated $\mathrm{C} 4$ to $\mathrm{rVCP}$. Results are represented by subtraction of the ligand channel and the control channel. The experiment was repeated to confirm results. 


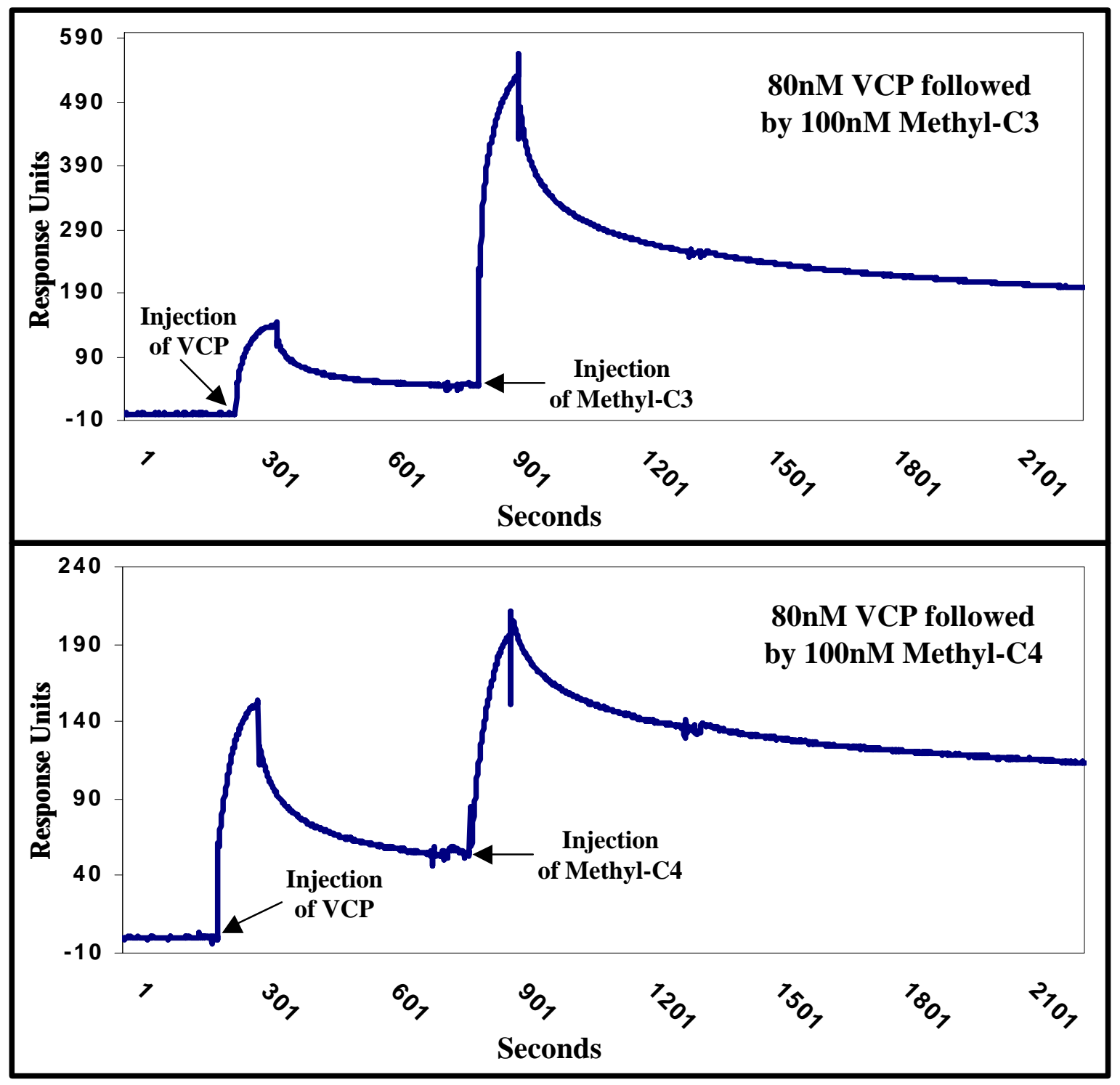


Figure 45. Model for simultaneous heparin and complement binding. Spatial relation of $\mathrm{C} 3 \mathrm{~b} / \mathrm{C} 4 \mathrm{~b}$ binding and heparin binding in $\mathrm{rVCP}$ is shown. The positive patch between modules 1 and 2 is shown with contributing basic residues labeled. Side chains of residues of putative importance for $\mathrm{C} 3 \mathrm{~b}$ and $\mathrm{C} 4 \mathrm{~b}$ binding by $\mathrm{rVCP}$ are shown as spheres on a $\alpha$-carbon trace (light blue) of rVCP. Color-coding is the same as in figure 46A. Heparin hexasacchride (carbon, brown; oxygen, red; nitrogen, blue and sulfur, yellow) derived from the complex with acidic FGF is also shown below with the four lysine residues (cyan) that modeling suggests are likely ligands. 


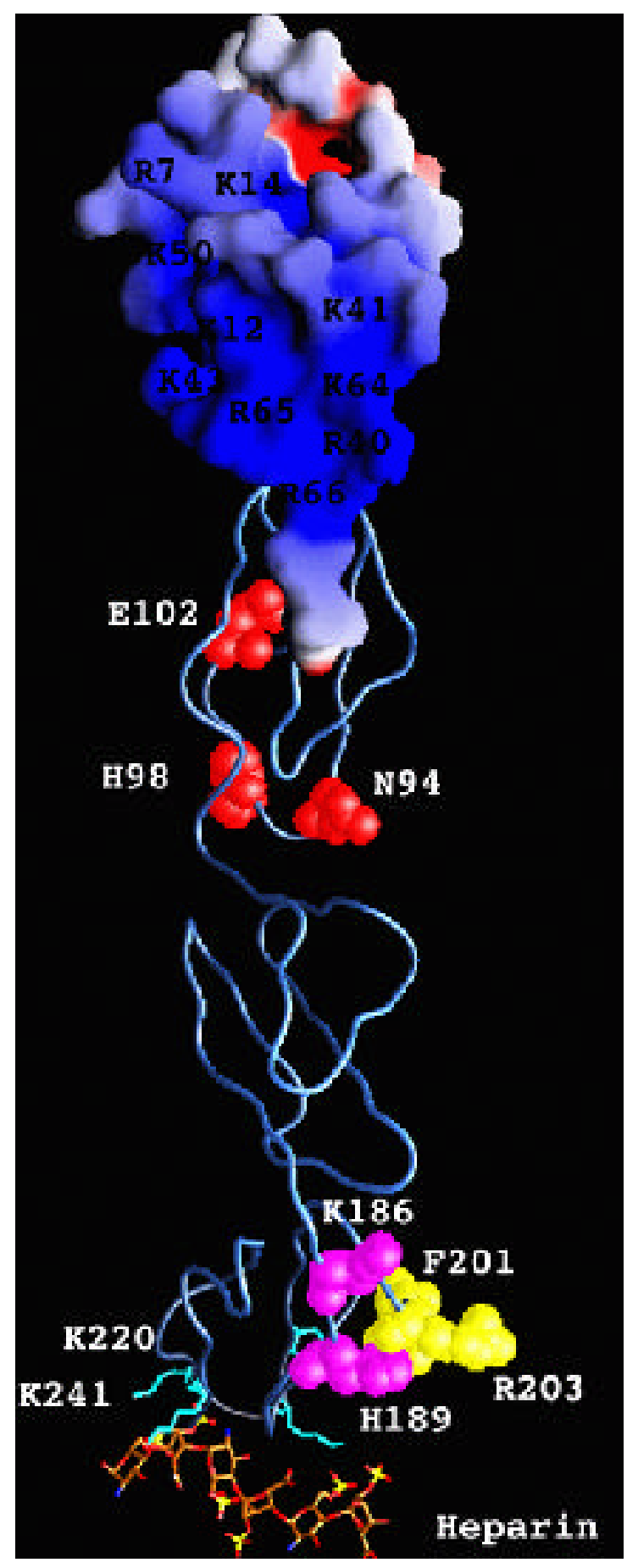


mutagenesis and epitope mapping of MCP is one of the most comprehensive (Liszewski et al., 2000). An alanine-scan of MCP from residues 94-103 (figure 46) proves to be particularly useful. The mutations Y97A, Y98A, L99A and E103A each abolished cofactor activity for C4b but not binding of C4b, while N94A, E95A, G96A and E102A decreased both $\mathrm{C} 4 \mathrm{~b}$ binding and cofactor activity, and $\mathrm{C} 3 \mathrm{~b}$ binding was affected only by E102A. The equivalent residues in VCP, also 94-103, are close in sequence (figure 46) and lie in module 2 near the interface with module 3 . Thus, by homology with the rVCP structure, it is likely that residues 94, 95, 98, 102 and 103 of MCP are involved directly, and N94, E95 and E102 directly contact C4b. It is reasonable to propose that N94, H98 and E102 (figures 45 and 46, red) play a similar role in rVCP. The same study (Liszewski et al., 2000) determined that residues in the region 191-196 of MCP were very important for C3b-binding. Substitutions of K193, R195 and F196 by alanine interfered with both C3b-binding and cofactor activity for C3b. In VCP, the equivalent region is 184-189 and lies at the C-terminus of module 3 with $\mathrm{K} 186$ and $\mathrm{H} 189$ exposed. Since K193 and F196 of MCP contact C3b, it is reasonable to propose that K186 and H189 of VCP (figures 45 and 46, magenta) play a similar role. Finally, in MCP, residues in the region 206-212, G207A, F208A, K210A, K211A and F212A are involved in C3bbinding since the mutations each significantly reduce affinity for C3b, but not C4b, F208 and K210 (figure 45, yellow) are likely to be contact points for C3b. In rVCP these residues lie within the sequence 199-205 of module 4 with F201 and R203 exposed at the surface. Although it is not unreasonable to invoke F201 and R203 (figures 45 and 46, yellow) as C3b-binding residues in rVCP, most of this module, including residues 208 
and 210 are missing from its MPV homologue and do not appear to be pivotal for this function. 
Figure 46. Putative C3/C4 binding residues in rVCP. A) Alignment of the sequences of $\mathrm{rVCP}$ and $\mathrm{MCP}$ in regions identified as potential $\mathrm{C} 3 \mathrm{~b} / \mathrm{C} 4 \mathrm{~b}$ binding sites are shown. The three sets of residues in MCP shown to decrease $\mathrm{C} 4 \mathrm{~b}$ binding (red), $\mathrm{C} 3 \mathrm{~b}$ binding (magenta and yellow) when mutated. rVCP residues in the three positions corresponding to those in MCP, which appear to be accessible for complement binding, are also shown in matching colors. B) Putative $\mathrm{C} 3 \mathrm{~b}$ and $\mathrm{C} 4 \mathrm{~b}$ binding regions of $\mathrm{VCP}$ are shown in alignment with a well-characterized C3b/C4b binding site on human MCP CCP 1 (shown outlined), along with other complement regulating proteins from different species. Noted in red are important residues, shown to be necessary for $\mathrm{C} 3 \mathrm{~b}$ binding by MCP. Colored green are highly conserved cysteine residues. The locations of each site are shown for comparison. 


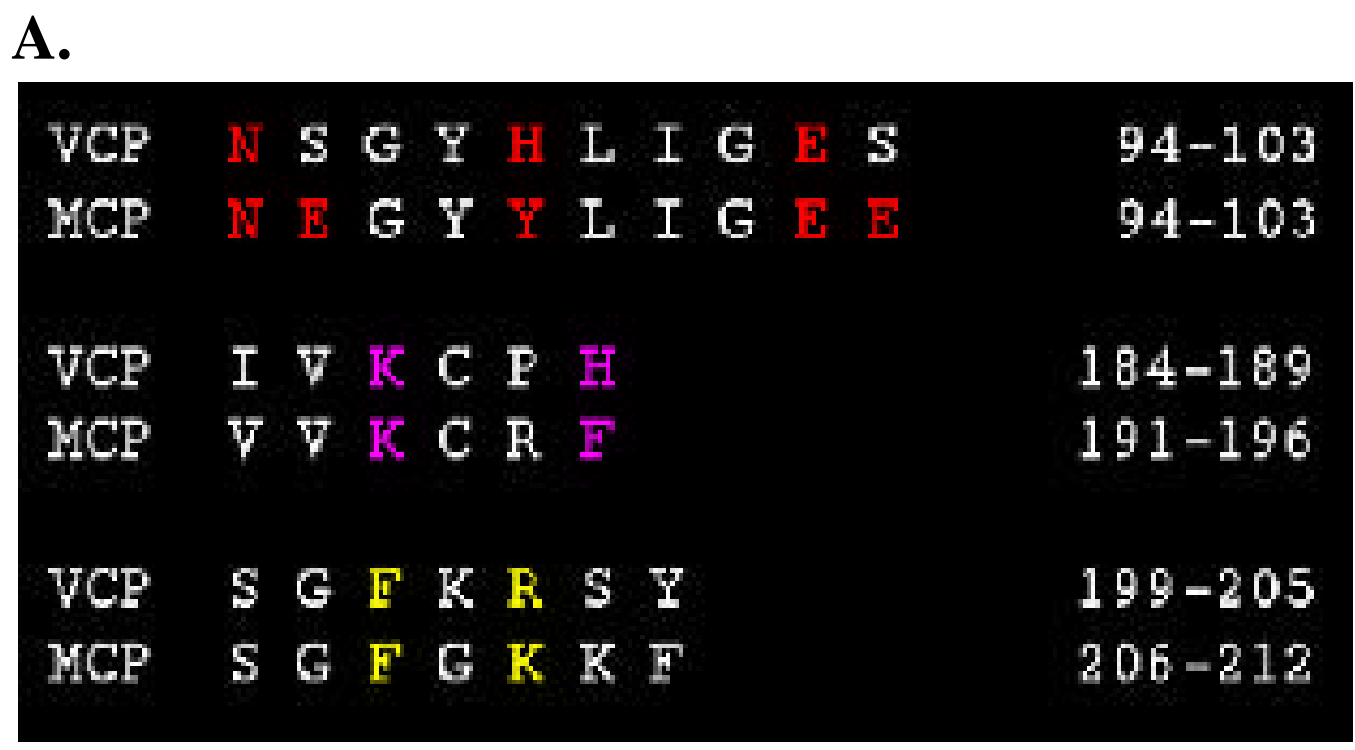

B.

Putitive C4b/C3b Binding Domains

On VCP

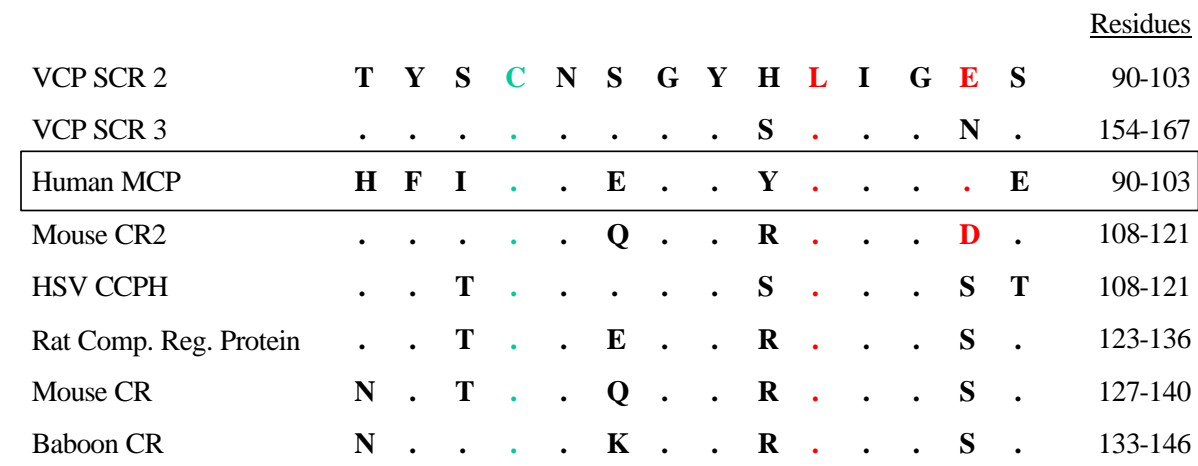

Proposed $\mathrm{C} 4 \mathrm{~b}$ binding domain of human MCP (outlined) is compared with similar sequences of other complement control proteins; conserved cysteine residues are colored green; important $\mathrm{C} 3 \mathrm{~b}$ contact residues are colored red. 


\section{$\underline{\text { Discussion }}$}

Results obtained by testing VCP's ability to inhibit the activation of complement, using various animal sera, demonstrate a significant difference in specificity. VCP was found to have very limited activity against rabbit complement, possessing 250 fold less activity than it has against rat sera (and possibly pig). It was also shown to possess moderate activity against human and baboon (data not shown) sera, having 4 to 8 fold less activity than against rat sera. Clearly, VCP shows the greatest activity against rat complement. This study does not suggest the reason for this significant difference in specificity. VCP may have a greater affinity for rat $\mathrm{C} 3 \mathrm{~b} / \mathrm{C} 4 \mathrm{~b}$, or its cofactor activity may be enhanced. The mechanism by which VCP blocks the activation of rat complement greater than human complement can be investigated further in the future by using Biacore (SPR) binding analysis. The affinity and kinetics of $\mathrm{VCP}$ with human or rat $\mathrm{C} 3 \mathrm{~b} / \mathrm{C} 4 \mathrm{~b}$ can be studied to help answer this question. The idea that VCP may be specific for rat, suggesting that a rat/rodent host coevolved with vaccinia virus, is further strengthened by studies done by Ariella Rosengard, University of Pennsylvania, on the variola virus homologue of VCP she termed SPICE, for smallpox inhibitor of complement enzymes. These studies suggest that the variola virus homologue inhibits complement activation of human sera fourfold more effectively than VCP. This suggests that SPICE is more specific for human complement due to it coevolving in a human host. This hypothesis is quite interesting considering that the difference between VCP and SPICE is only twelve residues.

In an attempt to map the location of $\mathrm{C} 3 \mathrm{~b} / \mathrm{C} 4 \mathrm{~b}$ binding and cofactor activity on $\mathrm{rVCP}$, various truncated proteins consisting of nearly all combinations of 2 and $3 \mathrm{CCP}$ 
module fragments [CCP modules 1 and 2 (rVCP 1,2), 2 and 3 (rVCP 2,3), 3 and 4 (rVCP 3,4), and 2-4 (rVCP 2,3,4)] were tested in the hemolysis assay. The results suggest that only full-length rVCP is able to inhibit complement activation in the hemolysis assay. To confirm these results using surface plasmon resonance (SPR), the recombinant truncated versions of $\mathrm{rVCP}$ were used to map the regions involved in $\mathrm{C} 3 \mathrm{~b}$ binding. Only full-length rVCP was able to bind C3b. These results help to confirm previous data showing that none of the mutant proteins were able to inhibit complementmediated lysis of sSRBCs in the hemolysis assay. The results of both studies suggest that VCP is the smallest functional unit, and, therefore, most of the exposed surface is required for binding complement, cofactor activity, and ultimately blocking complement activation. This was also suggested by previously published studies using truncated membrane bound versions of rVCP (Rosengard et al., 1999). With the origin of VCP in mind, it is easy to understand why this protein is the smallest functional unit. The size of the vaccinia virus genome, though it is quite large for a virus, is very limited in size. VCP was most likely pirated from the host, possibly as $\mathrm{C} 4 \mathrm{~b}-\mathrm{BP}$ or $\mathrm{MCP}$, and reduced to its minimal functional unit to conserve valuable genomic space. VCP does not represent the smallest $\mathrm{CCP}$ known to bind $\mathrm{C} 3 \mathrm{~b}$, only the smallest $\mathrm{CCP}$ able to bind $\mathrm{C} 3 \mathrm{~b}$ and inhibit complement activation. Surface plasmon resonance studies of fH have shown that a two CCP module fragment, SCR 19-20, was able to bind C3b (Jokiranta et al., 2001). Although this small fragment was able to bind both $\mathrm{C} 3 \mathrm{~b}$ and $\mathrm{C} 3 \mathrm{~d}$, it does not retain cofactor or decay accelerating activity (personal communication). The notion that a two $\mathrm{CCP}$ module fragment of $\mathrm{fH}$ is required to bind portions of $\mathrm{C} 3$, while all four CCPs of VCP are required, suggests that differing mechanisms of action are involved. The idea 
that VCP and fH may have different mechanisms of action to inhibit complement activation has been suggested by previous data (Sahu et al., 1998).

In order to investigate the hypothesis that VCP may inhibit complement activation while remaining bound to heparin/heparan sulfate, several experiments were employed. Various concentrations of heparan sulfate was added to a serially diluted sample of rVCP and then tested using the hemolysis assay. The ability of rVCP to inhibit complement activation was unchanged. This experiment was repeated using various concentrations of heparin with the same results. In order to be sure that rVCP was binding and remaining bound to heparin while being tested in the hemolysis assay, heparin sepharose beads were also used. rVCP bound to heparin sepharose beads was tested in various concentrations in the hemolysis assay. The results obtained by all experiments suggest that VCP is able to inhibit complement activation while remaining bound to heparin/heparan sulfate. These results were later confirmed using SPR analysis. With rVCP immobilized to the sensor chip, C3-MA or C4-MA was injected and binding between the proteins was confirmed. Next, with heparin immobilized to the sensor chip, rVCP was injected and allowed to bind before C3-MA or C4-MA was injected. Clear binding was observed between rVCP bound to heparin on the surface of the sensor chip and both C3-MA and C4-MA. These experiments imply that the protein, in addition to its ability to strongly inhibit complement activation while in soluble form, may also inhibit complement while bound to heparan sulfate proteoglycans lining the endothelial cell layer. rVCP would therefore be able to protect the infected host cell, and neighboring cells, from not only host complement attack, but also antibody binding and cell death caused by NK cells. The ability of rVCP to perform both functions at once has significant implications in our 
understanding of viral habitat formation. VCP may act in two states, the cell bound state, where it utilizes heparan sulfate to tether itself to the cell surface, and the soluble state, where it is free to diffuse into the surrounding local tissue. Therefore, VCP acting in the cell bound state may not only be involved in protecting the infected cell from the molecular and cellular interactions of the immune system, but also cell surface activation of complement. In addition, the soluble form of VCP may be able to diffuse locally, blocking complement activation in the surrounding tissue, and preventing the release of chemoattractant compounds. All together, the two states of VCP may create a habitat better suited for local spread of viral progeny and growth.

With the X-ray crystal structure of rVCP in hand (see chapter 5), the data obtained in the above experiments, as well as homology studies with other well characterized complement regulatory proteins, was used to map the locations of the complement binding regions and project them onto the structure of rVCP. MCP, whose residues involved in $\mathrm{C} 3 \mathrm{~b} / \mathrm{C} 4 \mathrm{~b}$ binding are well mapped, aligns very closely with $\mathrm{rVCP}$. Therefore, it was used to accurately predict the residues on $\mathrm{rVCP}$ involved in $\mathrm{C} 3 \mathrm{~b} / \mathrm{C} 4 \mathrm{~b}$ binding without requiring alanine-scanning mutagenisis of VCP. Taken together, the above experiments and homology data suggest that most, if not all, of the surface of $\mathrm{rVCP}$ is needed to bind $\mathrm{C} 3 \mathrm{~b} / \mathrm{C} 4 \mathrm{~b}$, as well as cofactor activity for their cleavage by factor I. VCP, made up of $4 \mathrm{CCP}$ modules, would represent the smallest functional unit required for binding and inhibiting complement activation. This knowledge could be important in the bioengineering of new therapeutic proteins capable of blocking complement activation. 


\section{CHAPTER 5}

\section{VCP STRUCTURE AND STABILITY}

\section{$\underline{\text { Introduction to CCP Structure }}$}

This chapter contains material adapted from the following publication and manuscript:

Murthy K.H., Smith S.A., Ganesh V.K., Judge K.W., Mullins N., Barlow P.N., Ogata C.M., and Kotwal G.J. Crystal structure of a complement control protein that regulates both pathways of complement activation and binds heparan sulfate proteoglycans. Cell. Jan. 26, 104:301-11, 2001.

Smith S.A., Krishnasamy G., Murthy K.H., Cooper A., Bromek K., Barlow P.N., and Kotwal G.J. Vaccinia virus complement control protein is monomeric, and retains structural and functional integrity after exposure to adverse conditions. Biochim. Biophys. Acta., In press, 2002.

As previously mentioned in chapter 4 , VCP exhibits sequence and functional similarity to the mammalian complement regulators, such as, factor $\mathrm{H}(\mathrm{fH})$, membrane cofactor protein (MCP), decay-accelerating factor (DAF), complement receptor type one (CR1), and complement $4 \mathrm{~b}$ binding protein $(\mathrm{C} 4 \mathrm{~b}-\mathrm{BP})$. $\mathrm{VCP}$ is most similar in terms of sequence to the $\mathrm{N}$-terminus of the $\alpha$-chain of $\mathrm{C} 4 \mathrm{~b}$ - $\mathrm{BP}$, sharing $24,33,35$ and 29 identical or conservatively replaced residues, out of about 60 , in modules 1, 2, 3 and 4, respectively (figure 32). VCP is the smallest, only 244 amino acids, and least complex of the complement control proteins, with no glycosylation or transmembrane domains, making it an excellent candidate for structural analysis. Structures of fragments of MCP with, two out of four CCP modules (Casasnovas et al., 1999) and fH with, one (Barlow et 
al., 1991; Barlow et al., 1992) or two (Barlow et al., 1993) out of 20 CCP modules have been published. The NMR structures of two pairs of modules of rVCP, modules 3 and 4 (rVCP 3,4) (Wiles et al., 1997) or 2 and 3 (rVCP 2,3) (Henderson et al., 2001), have also been published. None of these fragments shows complement inhibition activity or factor I cofactor activity. The structure of $b_{2}$-glycoprotein I ( $b_{2}$-GPI or apolipoprotein$\mathrm{H})$, a molecule with four CCP modules, has been reported, however, $\mathrm{b}_{2}$-GPI is not a complement inhibitor (Bouma et al., 1999; Schwarzenbacher et al., 1999). Thus, to date there has been no three-dimensional structure available of a protein or protein fragment that is able to regulate complement activation.

In all published structures of CCP modules, each folds into a compact 6-8 stranded $\beta$-structure that has a conformation similar to that originally observed in the structure of fH16 (Barlow et al., 1991). Between each module are "linkers" of varying lengths, which connect one module to another. The relative orientations of neighboring modules seem to differ from one module-pair to another quite significantly and in a manner that is not predictable from differences in primary sequences (Barlow et al., 1993; Casasnovas et al., 1999; Henderson et al., 2001; Gaboriaud et al., 2000). Despite this, bi-modular orientation preferences have been used in building structures containing three or more CCPs. Such models have been used to fulfill the need to provide a threedimensional context for functional and mutational studies of RCA proteins. Examples include models for the structures of rVCP and MCP (Liszewski et al., 2000). Similarly, homology modeling has been used to develop C3dg-binding bi-modular fragments of CR2 based on those identified on fH (Molina et al., 1995; Nagar et al., 1998; Prodinger et 
al., 1998). However, without the structure of a functional stretch of CCP modules, such models are only speculative.

The crystal structure of rVCP that is fully active both in complement inhibition and in binding heparin (in soluble form) was resolved in two different crystal forms by Dr. Krishna Murthy from the Center for Biophysical Science and Engineering, University of Alabama at Birmingham. This structure, for the first time, offers a picture of the threedimensional positioning of all the CCP domains necessary for a well-characterized complement inhibitory activity. Unlike studies of $\mathrm{CCP}$ protein fragments, this structure is not susceptible to the criticism that deletion of modules might alter orientations at inter-modular junctions. The high level of sequence similarity between VCP and the mammalian RCAs, as well as complement receptor type 2 (CR2) means that the rVCP structure will provide the richest and most reliable source of information yet. This information may be invaluable for modeling other functional stretches of three to four CCPs using homology, or for the design and interpretation of mutagenesis experiments aimed at understanding the structural basis of complement control. The structure of a fully active, complete four-domain rVCP presented here will significantly contribute to our understanding of viral and mammalian complement regulatory proteins, and ultimately many complement-mediated diseases.

Knowledge of the oligomerization state of VCP and its stability are crucial for understanding the durability of the evasion of host response mediated by VCP. Understanding the stability of VCP is also important for administration, shipping, storage, and dosage purposes. rVCP has now been shown, using gel filtration, to be a monomeric protein in solution. In order to test its thermal stability, the protein was 
examined by NMR over a range of temperatures and analyzed by differential scanning calorimetry. These studies demonstrate that the 3D structure of $\mathrm{rVCP}$ is robust and able to tolerate extreme temperatures. It was very important, however, to assess the extent to which functional activity is able to withstand high temperatures. Therefore, rVCP was subjected to a variety of harsh conditions, after which the specific activity was measured to determine whether the protein had retained its functional conformation. These included exposure to multiple freeze-thawing events and strong acids. rVCP was found to be functionally stable after exposure to severe conditions, remaining active even after boiling. The shelf-life of rVCP was also investigated and the protein was found to retain full activity at the longest time point tested. 


\section{Materials and Methods}

\section{Production and Purification of $r V C P$ :}

Samples of rVCP were produced using the Pichia pastoris yeast expression system (Invitrogen) and purified to greater than 95\% using heparin affinity chromatography (Pharmacia), described in chapter 2).

\section{X-Ray Crystal Structure of rVCP:}

The procedures for X-ray crystallography are described in chapter 3 .

\section{Shelf-life:}

Aliquots of $50 \mu \mathrm{l}$ of $\mathrm{rVCP}(2.75 \mu \mathrm{g} / \mu \mathrm{l})$ were placed in $0.65 \mathrm{ml}$ microcentrifuge tubes and stored at room temperature. At various time points, $2 \mu \mathrm{l}(5.5 \mu \mathrm{g})$ samples were tested for complement inhibition in the hemolysis assay. The experiment was performed on aliquots of a single rVCP sample.

\section{Freeze-thawing Sensitivity:}

A $0.5 \mathrm{ml}$ sample of $\mathrm{rVCP}(2.75 \mu \mathrm{g} / \mu \mathrm{l})$ was repeatedly frozen to $-20^{\circ} \mathrm{C}$ for $30 \mathrm{~min}$ and then rapidly thawed to room temperature. After various numbers of freeze-thaw events, $8 \mu \mathrm{l}$ was removed, serially diluted, and its activity tested in the hemolysis assay. The experiment was performed on a single rVCP sample. 


\section{Temperature Sensitivity:}

Aliquots of $50 \mu \mathrm{l}$ of $\mathrm{rVCP}(2.75 \mu \mathrm{g} / \mu \mathrm{l})$ were placed in $0.65 \mathrm{ml}$ microcentrifuge tubes and incubated in a heat block at various temperatures for 30 min. After incubation, $4 \mu \mathrm{l}(11 \mu \mathrm{g})$ was taken from the sample and tested in the hemolysis assay. The experiment was performed on aliquots of a single rVCP sample.

\section{pH Sensitivity:}

Several $100 \mu \mathrm{l}$ aliquots of $\mathrm{rVCP}(3.0 \mu \mathrm{g} / \mu \mathrm{l})$ were sealed in dialysis tubing and placed into flasks containing $150 \mathrm{ml}$ of water adjusted to various $\mathrm{pHs}$. After vigorous stirring for $30 \mathrm{~min}$, the dialysis tubing containing the sample of rVCP was removed and placed into a beaker with $800 \mathrm{ml}$ of water, to equilibrate the sample to a neutral $\mathrm{pH}$. After vigorous stirring for $1 \mathrm{~h}$, the sample was removed from the dialysis tubing and $4 \mu \mathrm{l}$ $(12 \mu \mathrm{g})$ was then tested by hemolysis assay. Several sentinel samples were removed early from the dialysis tubing and tested using litmus paper to ensure that the desired $\mathrm{pH}$ was achieved. The experiment was performed on aliquots of a single rVCP sample.

\section{Hemolysis Assay:}

The hemolysis assay was performed as described in chapter 2 .

\section{Gel Filtration:}

A 50 x $0.7 \mathrm{~cm}$ glass column was packed with $19 \mathrm{ml}$ Superdex 75 (Pharmacia) and equilibrated with either water or $100 \mathrm{mM} \mathrm{NaCl}$. Using blue dextran (Sigma), the column exclusion volume was determined. The positions of various gel filtration standards (4 mg 
each) (Sigma) were then determined individually by collecting numerous $100 \mu 1$ fractions, visualized by SDS-PAGE stained with coomassie blue, and quantitated by densitometry (AlphaImager 2000 software system). The position of $\mathrm{rVCP}(4 \mathrm{mg})$ was then determined, using the same procedure as the markers, and the densitometric readings superimposed with those of the markers. The whole process was performed in water and in $100 \mathrm{mM} \mathrm{NaCl}$ to prevent unwanted interactions with the column.

\section{Nuclear Magnetic Resonance (NMR):}

NMR measurements were performed by Paul Barlow from the Edinburgh Center for Protein Technology, University of Edinburgh, Edinburgh, UK, on a Varian (Palo Alto, CA, U.S.A.) Inova NMR spectrometer operating at $600 \mathrm{MHz}$ (proton frequency) in a 5 mm triple-resonance pulsed field gradient probe. The ${ }^{1} \mathrm{H}$ spectra were acquired using a pulse sequence with pulse field gradient water suppression using $0.3 \mathrm{mM}$ sample (Hwang and Shaka, 1998). Each of the spectra was acquired for $40 \mathrm{~min}$ at a range of temperatures from $37^{\circ} \mathrm{C}$ to $53^{\circ} \mathrm{C}$; an additional spectrum was measured at $37^{\circ} \mathrm{C}$ after the sample had been heated to $90^{\circ} \mathrm{C}$, as in the calorimetry measurement. Data were acquired, processed and plotted using VNMR (Varian VNMR 6.1, Varian Associates, Inc.).

\section{Differential Scanning Calorimetry (DSC):}

DSC studies were conducted by Paul Barlow from the Edinburgh Center for Protein Technology, University of Edinburgh, Edinburgh, UK, on a VP-DSC differential scanning calorimeter (Microcal, Northampton, MA, U.S.A.) with the assistance of Margaret Nutley (University of Glasgow, Glasgow, Scotland, U.K.). The cell volume 
was $1.5 \mathrm{ml}$, the rate of heating was $1^{\circ} \mathrm{C}$ per min, and excess pressure was kept equal to 80 $\operatorname{bar}\left(1 \mathrm{bar}=10^{5} \mathrm{~Pa}\right)$. Samples were equilibrated at $10^{\circ} \mathrm{C}$ and then heated to $90^{\circ} \mathrm{C}$. The partial molar heat capacity and melting curve were analyzed using standard procedures (Privalov and Potekhin, 1986). The collected data were processed using the software ORIGIN.2 (Microcal). The protein concentration was $0.016 \mathrm{mM}(0.46 \mathrm{mg} / \mathrm{ml}$; molecular mass $28.6 \times 10^{3} \mathrm{~g} / \mathrm{mol}$ ) as determined by measurements of absorbance at $280 \mathrm{~nm}$ according to a calculated extinction coefficient (Gill and von Hippel, 1989) of $44.2 \times 10^{3}$ $\mathrm{mol}^{-1} \mathrm{~cm}^{-1}$. 


\section{$\underline{\text { Results }}$}

\section{$\underline{X-R a y ~ C r y s t a l ~ S t r u c t u r e ~ o f ~} r V C P$ :}

In both the crystal forms of rVCP that were analyzed, the molecule has an elongated structure with no intramolecular contacts between non-sequential modules see figure 47. Packing of the two molecules (A and B) in the asymmetric unit of form I crystals ( $\mathrm{pH} 7.5$ ) is head-to-tail, with CCP module 4 of each molecule making contacts with module 1 of the other. In form II crystals ( $\mathrm{pH} 8.3)$ the three molecules ( $\mathrm{A}, \mathrm{B}$ and $\mathrm{C})$ in the asymmetric unit associate such that modules 3 and 4 of B interact with modules 1 and 2 of both $\mathrm{A}$ and $\mathrm{C}$. Packing interactions within the asymmetric units of the two crystal forms do not show evidence of specific oligomerization of rVCP molecules.

All five crystallographically independent molecules in the two structures are highly similar in structure as reflected in root mean square (rms) deviations between C $\alpha$ atoms in the $\beta$-strands of molecule $\mathrm{A}$ and those of $\mathrm{B}, \mathrm{C}, \mathrm{D}$ and $\mathrm{E}$ being $0.28,0.67,0.68$ and $0.69 \AA$, respectively - figure 48. At the module level, the originally observed $6 \beta$ strand topology is closely resembled by all CCP modules of both copies of rVCP within the asymmetric unit of form I crystals (Barlow et al., 1991; Barlow et al., 1992; Barlow et al., 1993). Module 1 is remarkable for its prominent "hypervariable" loop of 10 residues, K14-A23, compared to 2, 4 and 5 residues in modules 2, 3 and 4, respectively, projecting from rVCP near the N-terminal tip of the molecule. Also noteworthy in module 1 is an unusual insertion, Q42, $\mathrm{K} 43$, that forms a prominent $\beta$-bulge close to the interface with module 2. In addition, module 2 has a 5 residue insertion, L109-S114, that forms a long loop near the interface with module 1. 
Figure 47. Packing in form I and form II crystals. A) The three molecules in the asymmetric unit of form II crystals are shown with the domains color-coded as in panel B. Molecules A, B and C are labeled. Side chains of residues making polar interactions at intermolecular interfaces are shown. B) The two molecules in the asymmetric unit of form I crystals are shown. Domains 1-4 are colored red, blue, magenta and cyan respectively. Side chains of residues that form polar interactions at the two ends of the pair are also shown. Domains are marked D1-D4. 


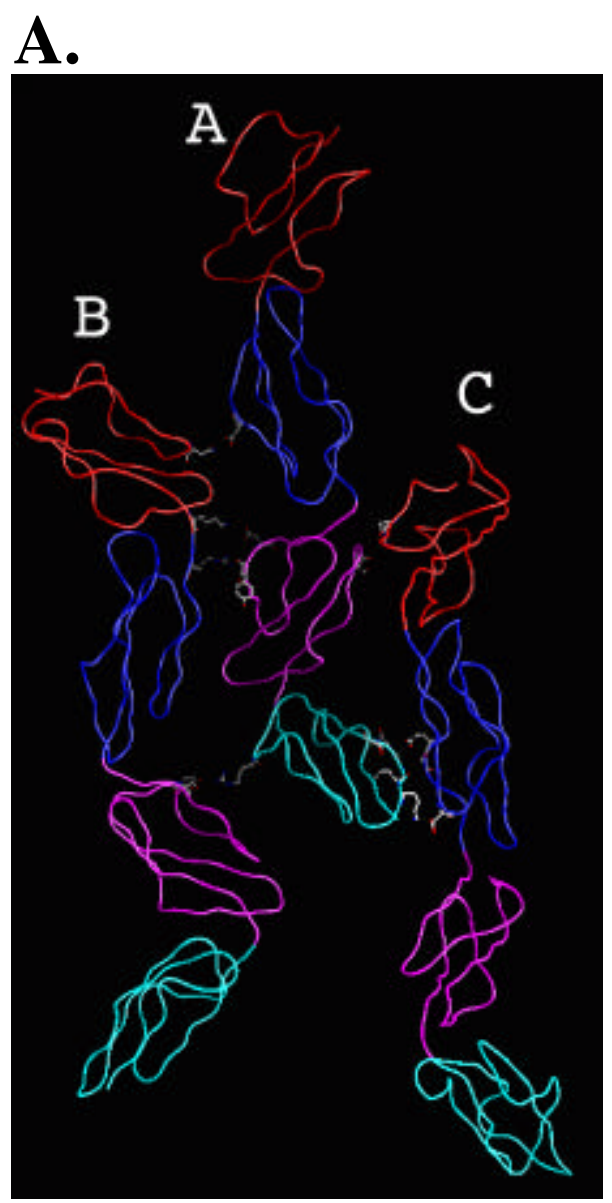

B.

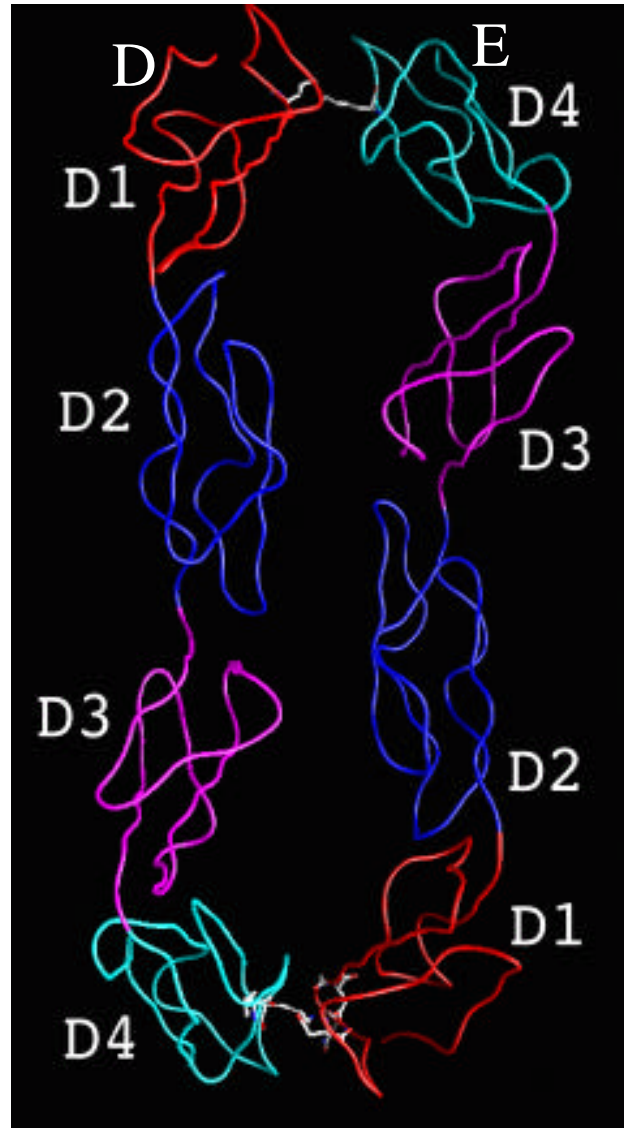


Figure 48. Five independent molecules are similar. a) Stereo pair of superposed C $\alpha$ traces of all five crystallographically independent molecules in the two crystal forms are shown. Molecule A in form I crystals is colored red, molecule B blue, and molecules C, $\mathrm{D}$ and $\mathrm{E}$ in form II crystals are colored magenta, cyan and yellow respectively. CCP domains are labeled mod1-mod4. b) A C $\alpha$ trace of molecule A from Form I crystals is shown in magenta in the same orientation as in panel a. Every $10^{\text {th }} \mathrm{C} \alpha$ has been colored yellow and numbered. The $\mathrm{N}$ and $\mathrm{C}$ terminal residues are shown in cyan and labeled. 

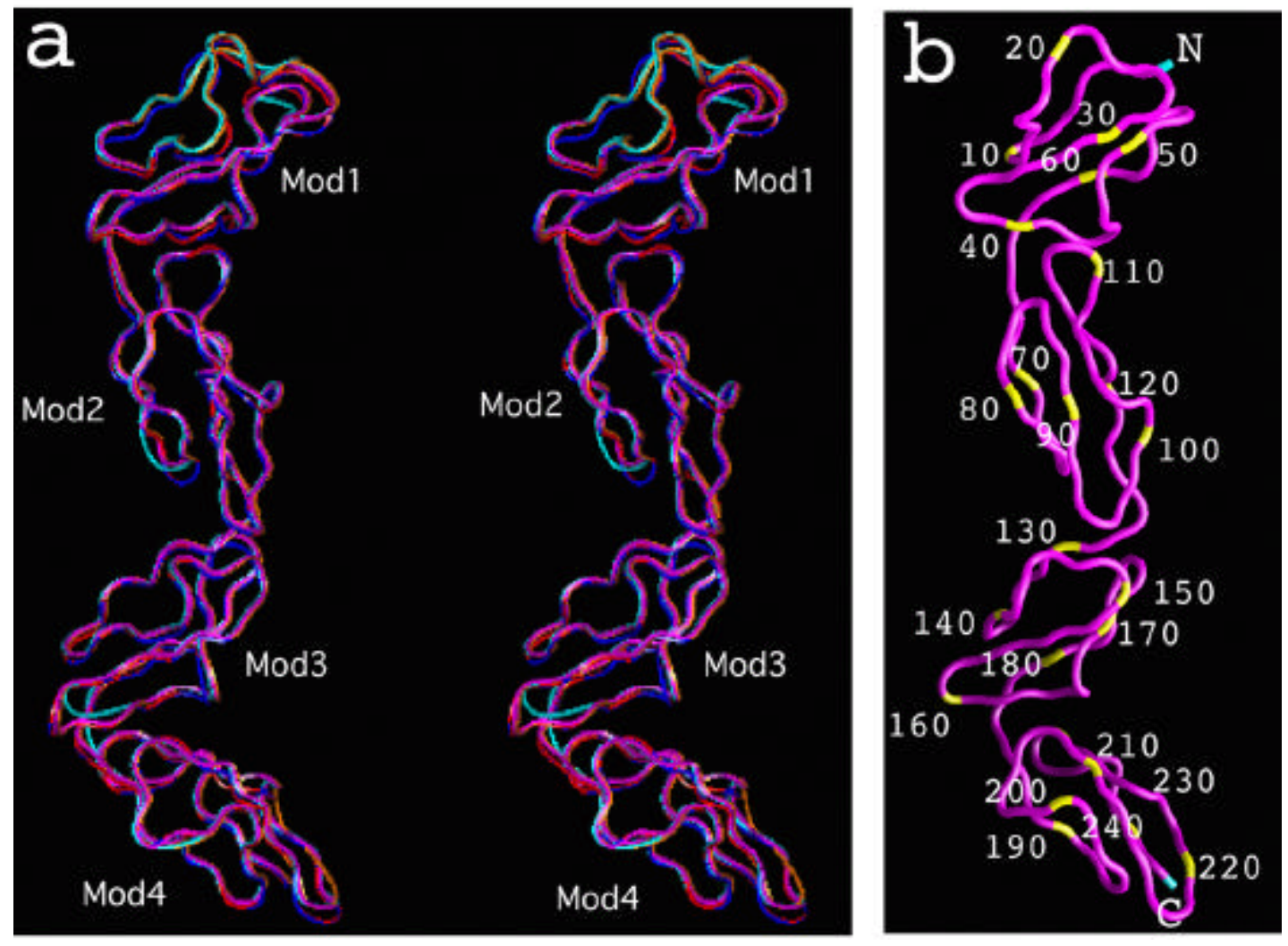


\section{VCP is a Monomer in Solution:}

In order to shed light on how VCP functions in vivo to maintain a habitat favorable to the virus, experiments were performed to determine whether it exists as a monomer or an oligomer in solution. Initial gel filtration experiments did not immediately determine whether rVCP was a monomer or multimer. As can be seen in figure 49a, one large peak and one small peak (possibly due to non-specific attachment to column matrix) can be seen in the rVCP densitometric profile. The presence of two distinct peaks is even more evident for the hemolysis activity of the fractions - figure 49b. However, when gel filtration was performed on marker proteins with and without $\mathrm{NaCl}, \mathrm{rVCP}$ was found to exist solely as a monomer in solution - see figure 50a and $\mathrm{b}$. In both cases, $\mathrm{rVCP}$ emerged from the column in approximately the same fractions as carbonic anhydrase, a $29 \mathrm{kDa}$ molecular weight marker protein. No sign of rVCP was found in the 60 or $90 \mathrm{kDa}$ range, where it may have emerged if it had been a dimer or trimer.

\section{Shelf-life and Freeze-thawing Tolerance:}

In order to test the shelf-life of rVCP, several $50 \mu \mathrm{l}$ aliquots $(2.75 \mu \mathrm{g} / \mu \mathrm{l})$ of $\mathrm{rVCP}$ (in $18 \mathrm{M} \Omega$ ultrapure water) were allowed to sit at room temperature for various periods of time prior to assay of hemolysis inhibiting activity. As shown in figure 51b, rVCP continued to remain fully active after 30 days, the longest time point tested. This demonstrates that rVCP in solution has a long shelf-life. In addition, rVCP was tested to determine its ability to resist irreversible denaturation caused by multiple freeze-thawing events. A $0.5 \mathrm{ml}$ sample $(2.75 \mu \mathrm{g} / \mu \mathrm{l})$ of $\mathrm{rVCP}$ was freeze-thawed fifty times. Figure $52 \mathrm{a}$ 
Figure 49. Gel filtration chromatography of rVCP. A) Gel filtration fractions containing rVCP (fractions 67-103) are visualized by SDS-PAGE and coomassie blue staining. Densitometry shows the existence of at least two peaks. Gel filtration chromatography was performed in water. B) Each fraction was tested for hemolysis inhibiting activity in the hemolysis assay. Due to the sensitivity of this assay, the peaks are easily visualized. The same fractions are shown from top to bottom of the figure. 


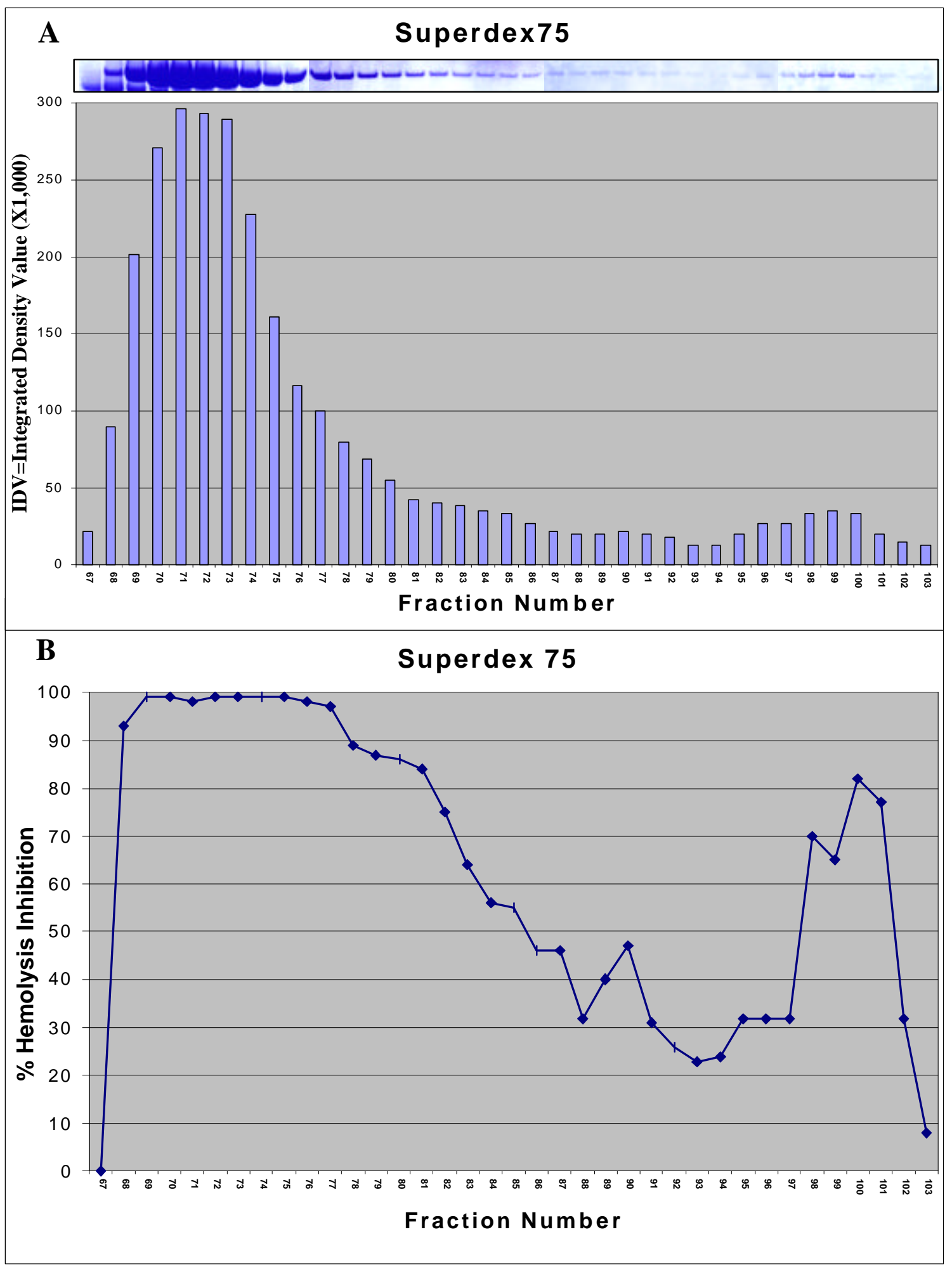


Figure 50. Gel filtration chromatography of rVCP and marker proteins. a) Overlaid densitometric results of gel filtrations conducted on rVCP and marker proteins ranging from $200 \mathrm{kDa}$ to $12.4 \mathrm{kDa}$. Column and samples were equilibrated in water. The second rVCP peak observed in figure 49 does not appear on this plot (smaller than $12 \mathrm{kDa}$ ) and is most likely an artifact. b) Overlaid desitometric results of gel filtrations conducted on rVCP and marker proteins ranging from $66 \mathrm{kDa}$ to $12.4 \mathrm{kDa}$. Column and samples were equilibrated in $100 \mathrm{mM} \mathrm{NaCl}$. 


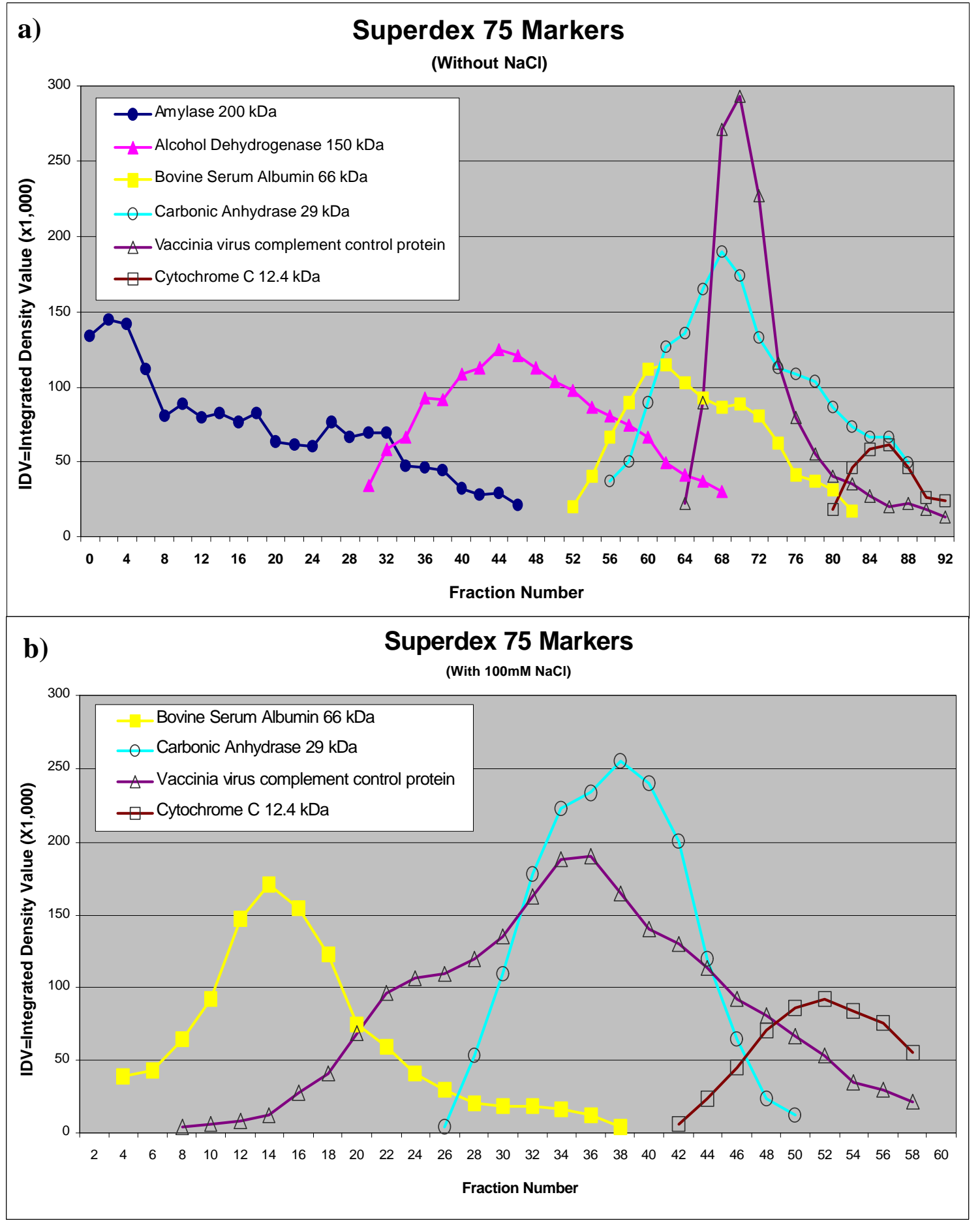


Figure 51. rVCP tolerance to extreme temperature and shelf-life. a) Hemolysis inhibiting activity of $11 \mu \mathrm{g} \mathrm{rVCP}$ taken from samples that were incubated for $30 \mathrm{~min}$ at temperatures ranging from 37 to $115^{\circ} \mathrm{C}$. b) Hemolysis inhibiting activity of $5.5 \mu \mathrm{g} \mathrm{rVCP}$ taken from samples that were incubated at room temperature $\left(\sim 25^{\circ} \mathrm{C}\right)$ for $6 \mathrm{~h}, 12 \mathrm{~h}, 24 \mathrm{~h}$, 48 h, 72 h, 1 week, or 1 month. 

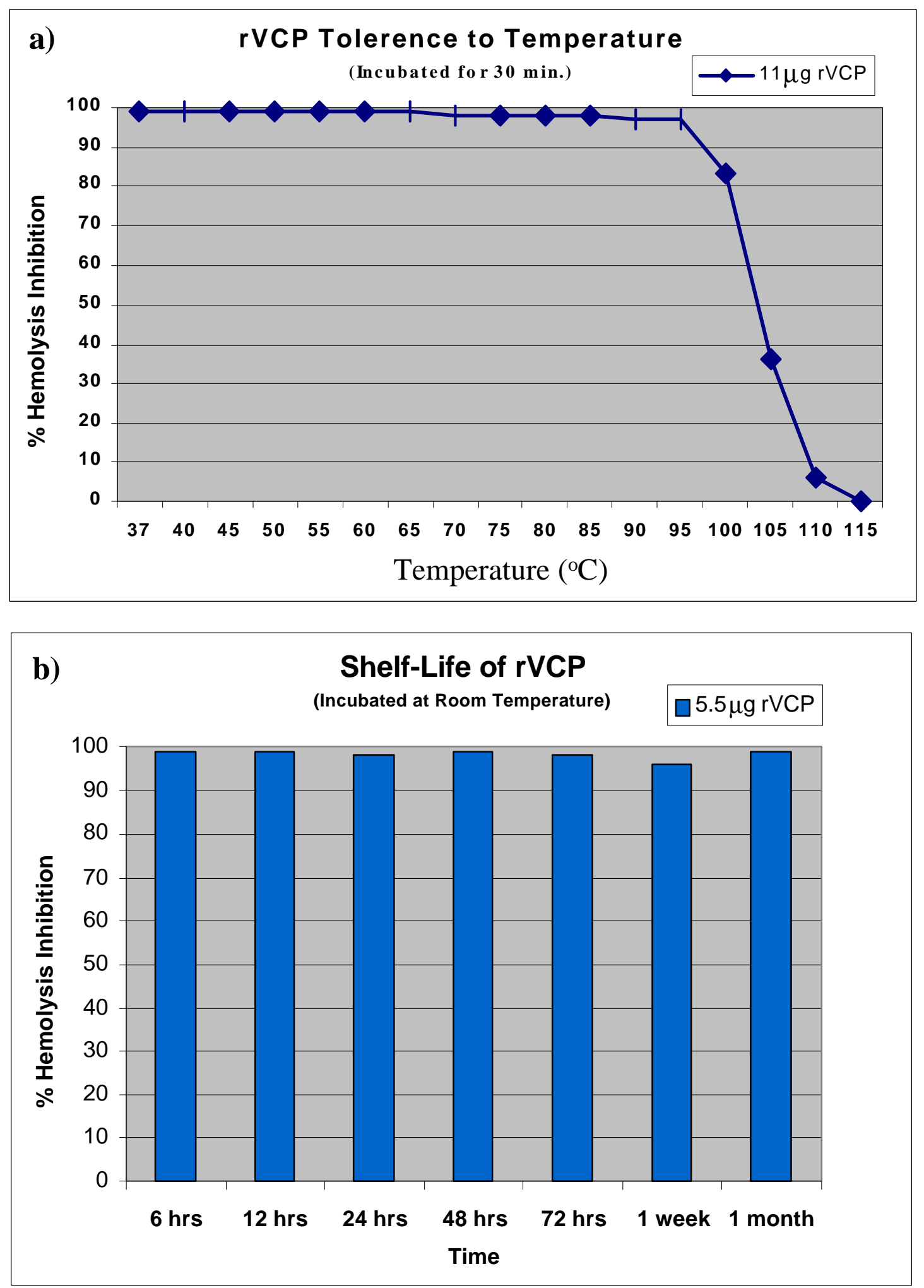
Figure 52. rVCP tolerance to freeze-thawing and $\mathbf{p H}$. a) Hemolysis inhibiting activity of serially diluted $\mathrm{rVCP}(2.75 \mu \mathrm{g} / \mu \mathrm{l})$ that has been freeze-thawed $2,5,10,25$, or 50 times. Also shown is serially diluted $\operatorname{rVCP}(2.75 \mu \mathrm{g} / \mu \mathrm{l})$ that was lyophilized and resuspended to the original concentration. b) Hemolysis inhibiting activity of $12 \mu \mathrm{g}$ rVCP taken from samples that were adjusted to a wide range of $\mathrm{pH}$ for $30 \mathrm{~min}$, and returned to neutral. 

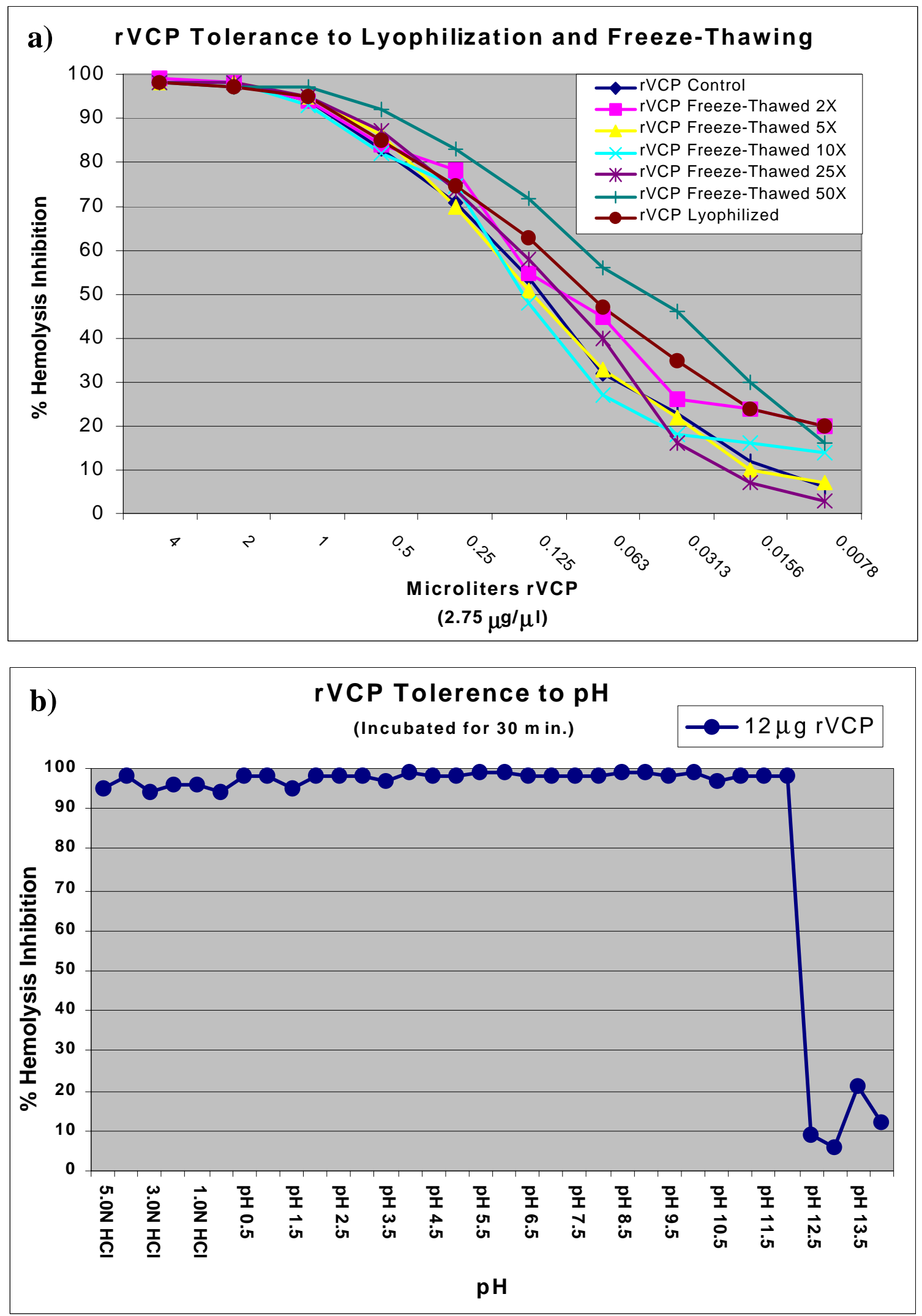
shows that rVCP is completely tolerant to freeze-thawing, maintaining complete activity after fifty events. A slight enhancement in activity appears when rVCP was tested after fifty freeze-thawing events. This is most likely due to evaporation taking place over the course of the experiment. As also shown in figure 52a, rVCP was found to be completely tolerant to lyophilization. The ability of this novel immunomodulating protein to tolerate freeze-thawing, lyophilization, and long periods of time at room temperature, will prove very valuable for shipping and storage purposes.

\section{Temperature and $p H$ Tolerance:}

To assess the extent to which functional activity is to withstand high temperatures, $50 \mu \mathrm{l}$ samples of protein in water $(2.75 \mu \mathrm{g} / \mu \mathrm{l})$ were incubated at various temperatures for 30 minutes prior to testing of their activity $\left(\right.$ at $\left.37^{\circ} \mathrm{C}\right)$ using the hemolysis assay. As can be seen in figure 51a, rVCP retains most of its activity after exposure to temperatures up to the boiling point of water. At $100^{\circ} \mathrm{C}$ only a slight white precipitate was evident. At $110^{\circ} \mathrm{C}$, however, most of the protein was irreversibly denatured and was seen to form a white precipitate. It may be possible that sample dilution during assaying allowed for a small amount of aggregated protein to re-dissolve and regain functional activity. Nonetheless, the results of these experiments reveal the high structural and functional stability of rVCP.

To assess the ability of rVCP to tolerate extremes in $\mathrm{pH}, 100 \mu \mathrm{l}$ aliquots of protein were sealed in dialysis tubing and the $\mathrm{pH}$ of the sample changed. After returning the $\mathrm{pH}$ of the sample to near neutrality, the hemolysis inhibiting activity of the protein was tested, see figure 52b. Recombinant VCP was able to withstand exposure to a pH 
well below zero, at which point the dialysis tubing was destroyed $(>5.0 \mathrm{~N} \mathrm{HCl}) . \mathrm{rVCP}$, however, was not quite as stable under basic conditions, becoming irreversibly denatured and forming a heavy white precipitate at $\mathrm{pH} 12.5$. The extreme acid stability of rVCP may be very important for administration purposes. Since rVCP should easily be able to tolerate the acidity of the stomach, oral administration of this complement inhibitor may be possible.

\section{Nuclear Magnetic Resonance (NMR) and Differential Scanning Calorimetry (DSC)}

\section{Studies:}

The thermal stability of rVCP was studied using differential scanning calorimetry. Figure 53a shows the calorimetric profile, which can be fitted with three, two-state, fully reversible transitions. That the curve may be fitted in this way implies that van't Hoff and calorimetric enthalpies are equal; attempts at fitting without this restraint produced an order of magnitude difference between the enthalpies, which could not be explained by any reasonable transition model (Privalov and Potekhin, 1986). The three fitted transitions are characterized by midpoint temperature, $\mathrm{T}_{\mathrm{m}}=58.3 \pm 0.07^{\circ} \mathrm{C}$ with enthalpy, $\Delta \mathrm{H},=71.0 \pm 0.7 \mathrm{kCal} \mathrm{mol}^{-1}, \mathrm{~T}_{\mathrm{m}}=62.68 \pm 0.09^{\circ} \mathrm{C}$ with $\Delta \mathrm{H}=84.2 \pm 0.6 \mathrm{kCal} \mathrm{mol}^{-1}$ and $\mathrm{T}_{\mathrm{m}}=68.46 \pm 0.10^{\circ} \mathrm{C}$ with $\Delta \mathrm{H}=62.4 \mathrm{e} \pm 7.1 \mathrm{kCal} \mathrm{mol}^{-1}$. As the protein consists of four modules it is possible that more transitions could be invoked, however these three were sufficient to represent the experimental profile. We are therefore unable to assign discrete transitions to individual modules. The calorimetry indicates that the thermal unfolding is to a large extent reversible as may be judged from the fitting but also from the repeated scan - see figure 53b. Although in the repeated scan there is some loss of 
Figure 53. Differential scanning calorimetry profiles. A) The experimental data points after baseline subtraction are shown as black dots. Solid thin line indicates reconstructed profile using three fitted transitions represented as dotted lines. B) The experimental data from following scan with data points represented as in A. 


\section{Differential Scanning Calorimetry}
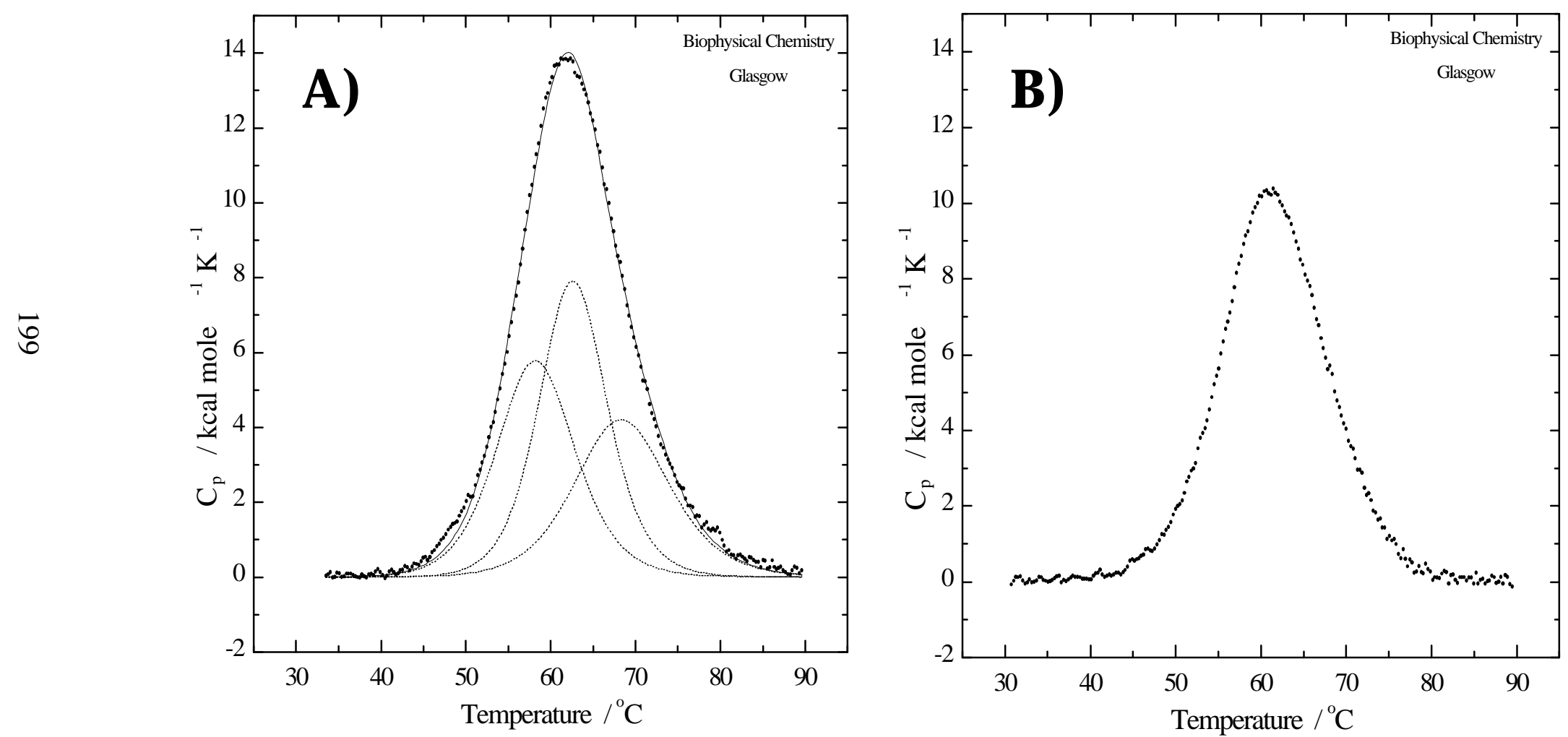
enthalpy it is consistent with most of the protein molecules refolding and just a small proportion of the population not reaching the lowest energy state. The current results may be compared with previous data reported for $\mathrm{rVCP} 2,3$, i.e. a protein fragment containing just the two central modules of VCP (Kirkitadze et al., 1999). The calorimetric profile of $\mathrm{rVCP} \sim 2,3$ showed a $\mathrm{T}_{\mathrm{m}}=57^{\circ} \mathrm{C}$ and $\Delta \mathrm{H}=86 \mathrm{kCal} \mathrm{mol}^{-1}$. The curve for $\mathrm{rVCP} 2,3$ had a distinct shoulder and could not be fitted to one or more twostate reversible transitions.

To further explore structural properties of this protein during heating and cooling, a series of $1 \mathrm{D}{ }^{1} \mathrm{H}$ NMR spectra was acquired over a range of temperatures. These show that no significant changes appear between the physiological temperature of $37^{\circ} \mathrm{C}$ and the elevated temperature of $53^{\circ} \mathrm{C}$ (the maximum that could safely be achieved in the NMR probe) - compare figure 54a and b. In contrast, NMR data obtained previously on $\mathrm{rVCP} 2,3$ showed that module 2 melted in the range $45-55^{\circ} \mathrm{C}$, although module 3 was more stable. The NMR data for $\mathrm{rVCP} \sim 2,3$ were consistent with its calorimetric profile. Clearly, module 2 is more stable in the context of full-length rVCP than in the 2,3 fragment. To investigate the structural properties of the protein after the reversible unfolding step, a spectrum at $37^{\circ} \mathrm{C}$ after heating to $90^{\circ} \mathrm{C}$ was acquired. The full ${ }^{1} \mathrm{H}$ spectrum is shown as an inset in figure 54c. All of the spectral lines in the methyl region of the ${ }^{1} \mathrm{H}$ NMR spectrum, which are sensitive to $3 \mathrm{D}$ structural integrity of the protein, are retained after heating and cooling - see figure 54c. 
Figure 54. NMR spectroscopy. a) and b) ${ }^{1} \mathrm{H}$ NMR spectra of VCP at $37^{\circ} \mathrm{C}$ and $53^{\circ} \mathrm{C}$ respectively. c) Overlaid methyl regions of ${ }^{1} \mathrm{H}$ NMR spectra of VCP as aquired before, and after heating to $90^{\circ} \mathrm{C}$ showing evidence of folded protein. Inset shows entire ${ }^{1} \mathrm{H}$ spectrum of VCP as acquired after heating to $90^{\circ} \mathrm{C}$. 


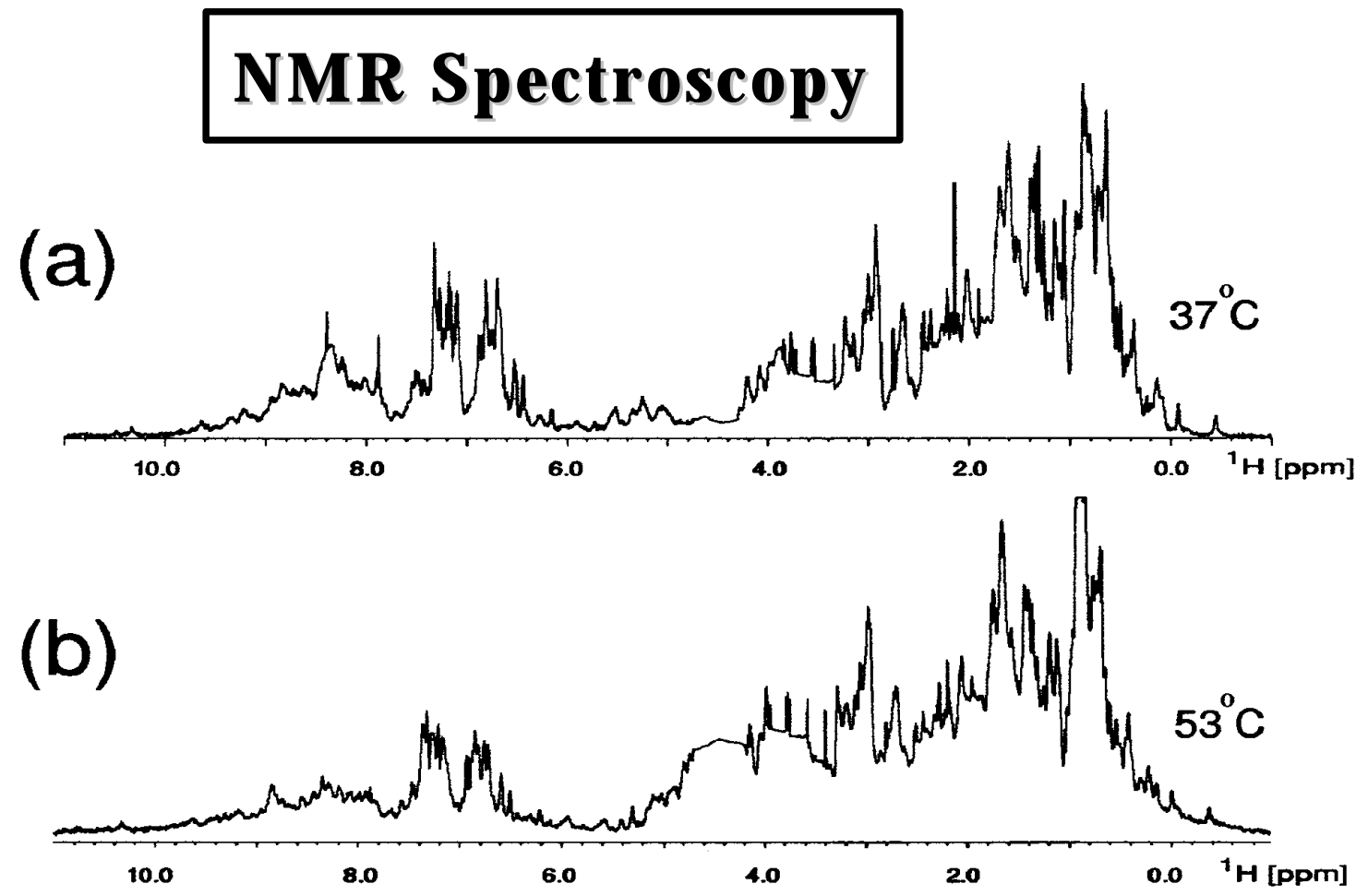

(c)

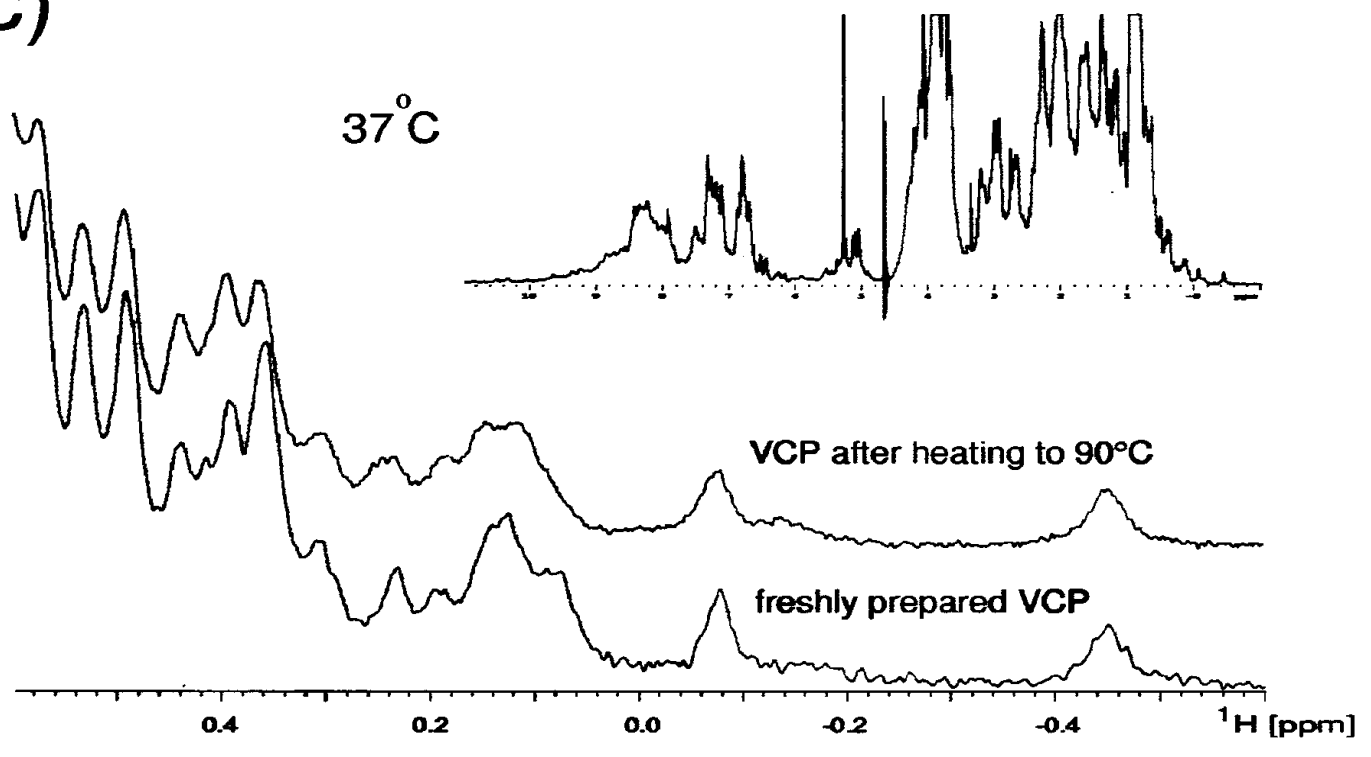




\section{$\underline{\text { Discussion }}$}

As suggested by the root mean square (rms) deviations between $\mathrm{C} \alpha$ atoms in the $\beta$-strands shown in figure 48 , the relative orientations of each of the four modules of rVCP are remarkably similar among all five molecules in the two crystal forms (2 in I and 3 in II) despite their being crystallographically independent. This suggests that this conformation is strongly preferred of rVCP in solution under the conditions used to grow the crystals, and that packing interactions are not responsible for the conformation observed. The "tilt" angles (i.e. the rotation required to align the z-axis of the inertia tensor of each module with its neighbor) between the long axes of the modules are $60^{\circ}$, $63^{0}$ and $99^{0}$ at the 1-2, 2-3 and 3-4 module junctions, respectively. However, the tilts between adjacent pairs of modules are in almost diametrically opposite directions, so that module 3 is tilted by only $10^{\circ}$ relative to module 1 and module 4 is tilted by $36^{\circ}$ relative to module 2, forming a "W" appearance. Thus, the molecule has an extended and approximately linear part consisting of modules 1-3, then a kink between modules 1-3 and module 4. Interactions at the 1-2 interface in rVCP are between mostly hydrophilic residues, those at the 2-3 interface are mixed, and those at the 3-4 interface are predominantly hydrophobic.

Figure 55a superimposes the structures of VCP and MCP 1,2 (Casasnovas et al., 1999). The arrangement of the N-terminal modules of MCP differs from that found in rVCP, providing another example of the significant variation in inter-modular orientations among this family of proteins. Knowing the crystal structure of rVCP, comparisons between the structure of intact rVCP and the solution structures of rVCP fragments, conducted previously, can be made - also shown in figure 55a. The angles 
Figure 55. Diverse orientations of modules in CCP containing proteins. The four modules of rVCP are shown in red and labeled. a) The first CCP module of MCP (gold) is superimposed over module 1 of $\mathrm{rVCP}$ using the $\beta$ sheet residues in each to calculate the transformation. Similarly, module 2 of the solution structure of $\mathrm{rVCP} 2,3$ (rose) is superimposed over that of VCP2 and module 3 of the solution structure of VCP3,4 (purple) superimposed over module 3 of rVCP. b) The first CCP module of rVCP is superimposed over module 1 of $\beta_{2}$ GPI (light blue). Modules 1-4 in each are labeled. Not shown is an additional membrane association domain beyond domain 4 in $\beta_{2} \mathrm{GPI}$. 
a

b
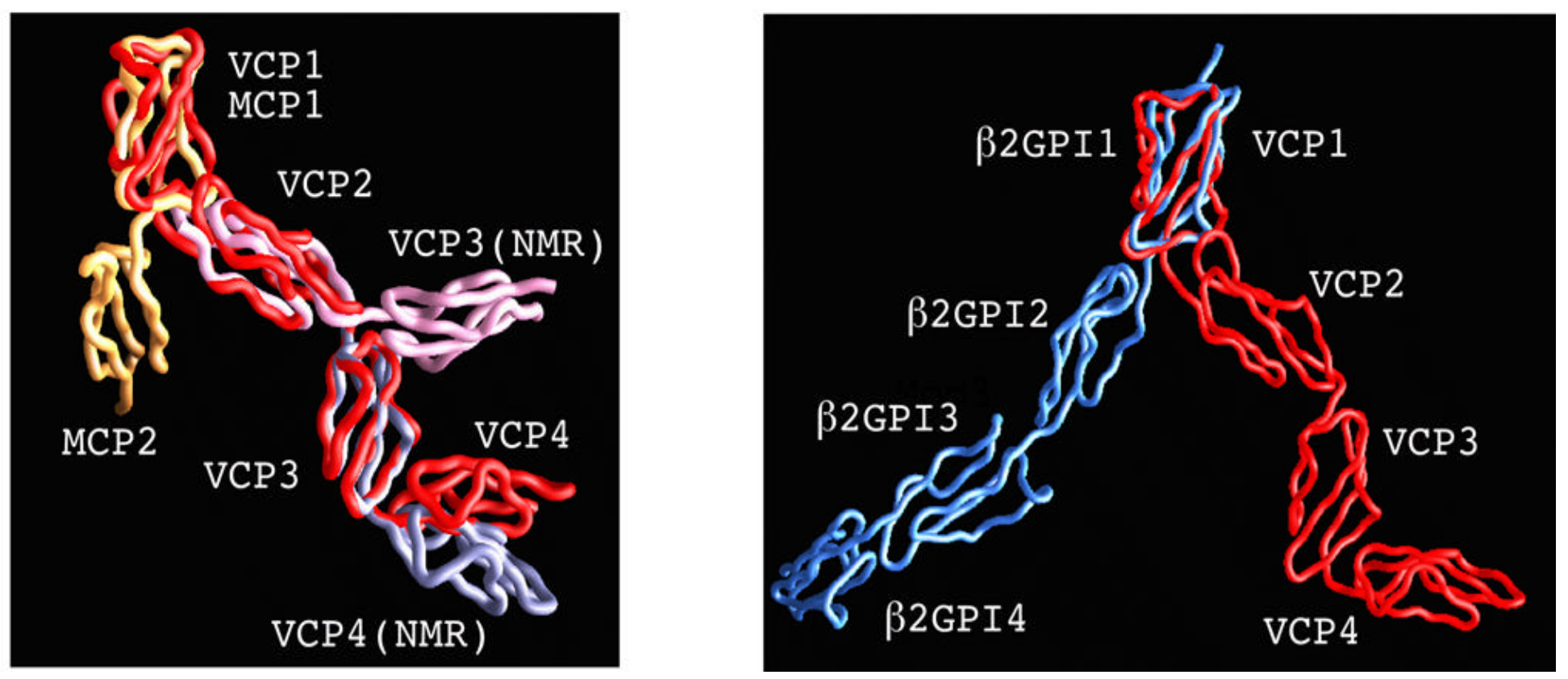
between modules 3 and 4 of the NMR structure of $\mathrm{rVCP} 3,4$ (tilt $=67^{\circ}$, twist $=81^{\circ}$, and skew $=7^{\circ}$ ) are comparable to those seen in the crystal structure (tilt $=99^{\circ}$, twist $=74^{\circ}$ and skew $\left.=-20^{\circ}\right)($ Wiles et al., 1997). The 2-3 intermodular angles within the NMR structure of $\mathrm{rVCP} 2,3$, however, are dissimilar to those in the crystal structure of intact rVCP. Moreover, approximately $281 \AA^{2}$ of surface is buried between modules 2 and 3 in the crystal form, while the junction between these two modules is not extensive in the NMR rVCP 2,3 fragment (Kirkitadze et al., 1999). In addition, NMR indicates that this pair is very flexible (Henderson et al., 2001). It is noticeable, however, that there are significant differences in the structure of module 2 between intact and truncated forms of rVCP, while modules 3 and 4 show only small insignificant differences. The notable differences observed in module 2 may be the result of the lack of module 1 , which could destabilize module 2 in $\operatorname{rVCP} 2,3$, and hence the 2,3 junction. For example, the L109S114 insertion in module 2 is a mobile loop in rVCP 2,3 but has moved by some $15 \AA$ in the crystal structure and contacts the Q42, K43 insertion of module 1. Modules 2 and 3 might also stabilize each other, which could explain the smaller differences between the arrangement of modules 2 and 4 in intact rVCP compared to the $\mathrm{rVCP} 3,4$ fragment. It is clear from these comparisons that the structure of rVCP reported here, the first of an intact complement control protein, represents a significant advance over previous structural studies based on fragments. This structure's validity does not rely on the assumption that CCP modules are structurally independent of the remainder of the protein. Therefore, this structure may be considered the most reliable basis yet for the design of mutagenesis experiments and homology modeling exercises. 
Previous data suggested that VCP might function as an oligomer in order to increase its stability and life in solution (Kotwal et al., 1990). Furthermore, dimeric and trimeric structures are observed in crystals. Current results suggest that rVCP may be found as a monomer in its soluble state, where it is able to diffuse throughout the area of viral infection, binding and destroying complement in the local surrounding tissue. rVCP also exists in the cell bound state, where it remains bound to heparan sulfate found on the infected cell, and neighboring cells' surfaces. In this state, rVCP is able to prevent complement-mediated cell lysis, as well as chemotactic migration of monocytes, NK cells and neutrophil-mediated cell killing, and antibody attachment. It is not known if rVCP oligomerizes at the cell surface. Additional gel filtration experiments are needed to determine whether rVCP can oligomerize in the presence of low molecular-weight heparin.

The ability of rVCP to resist irreversible denaturation in extremes of heat and $\mathrm{pH}$ can be attributed to its unusual architecture. The protein is composed of four modules arranged in an extended head-to-tail fashion. Each module has an elongated shape and is composed of extended regions of peptide running approximately parallel and antiparallel with the long axis, with loops and turns formed at either end of the module. The main chain makes a total of five passes up and down the module such that the $\mathrm{N}$-terminus is at one end of the long axis and the C-terminus is at the other. The extended regions are connected by networks of hydrogen-bonds (H-bonds) and over much of their length form $\beta$-strands that lie in antiparallel double or triple-stranded $\beta$-sheets - see figure 56 a. In addition to interstrand $\mathrm{H}$-bonds, two disulphide bonds within each module provide tethers, holding the module together. The disulphides involve the four absolutely 
Figure 56. Extensive H-bonding within VCP structure contributes to its stability. a) $\mathrm{C} \alpha$ trace (shown in red) of the VCP molecule crystal forms I and II (13) with main-chain H-bonds (shown in white). b) Residues $10 \AA$ around the center of each module interface are shown with H-bonds (shown in red). The main-chain hydrogen bonds were shown in white (b1) module 1 - module 2 interface (b2) module 2 - module 3 interface (b3) module 3 - module 4 interface. 


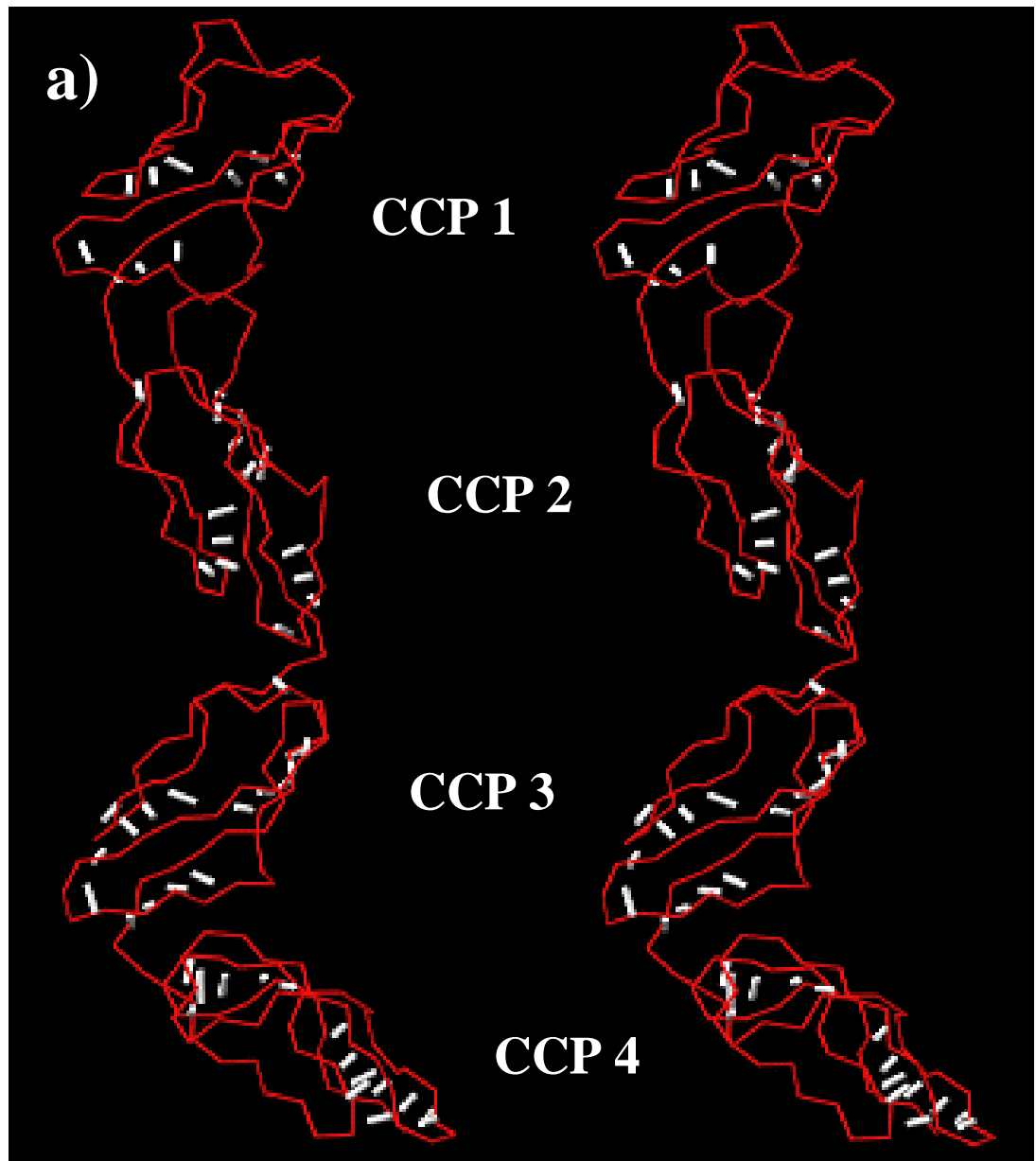




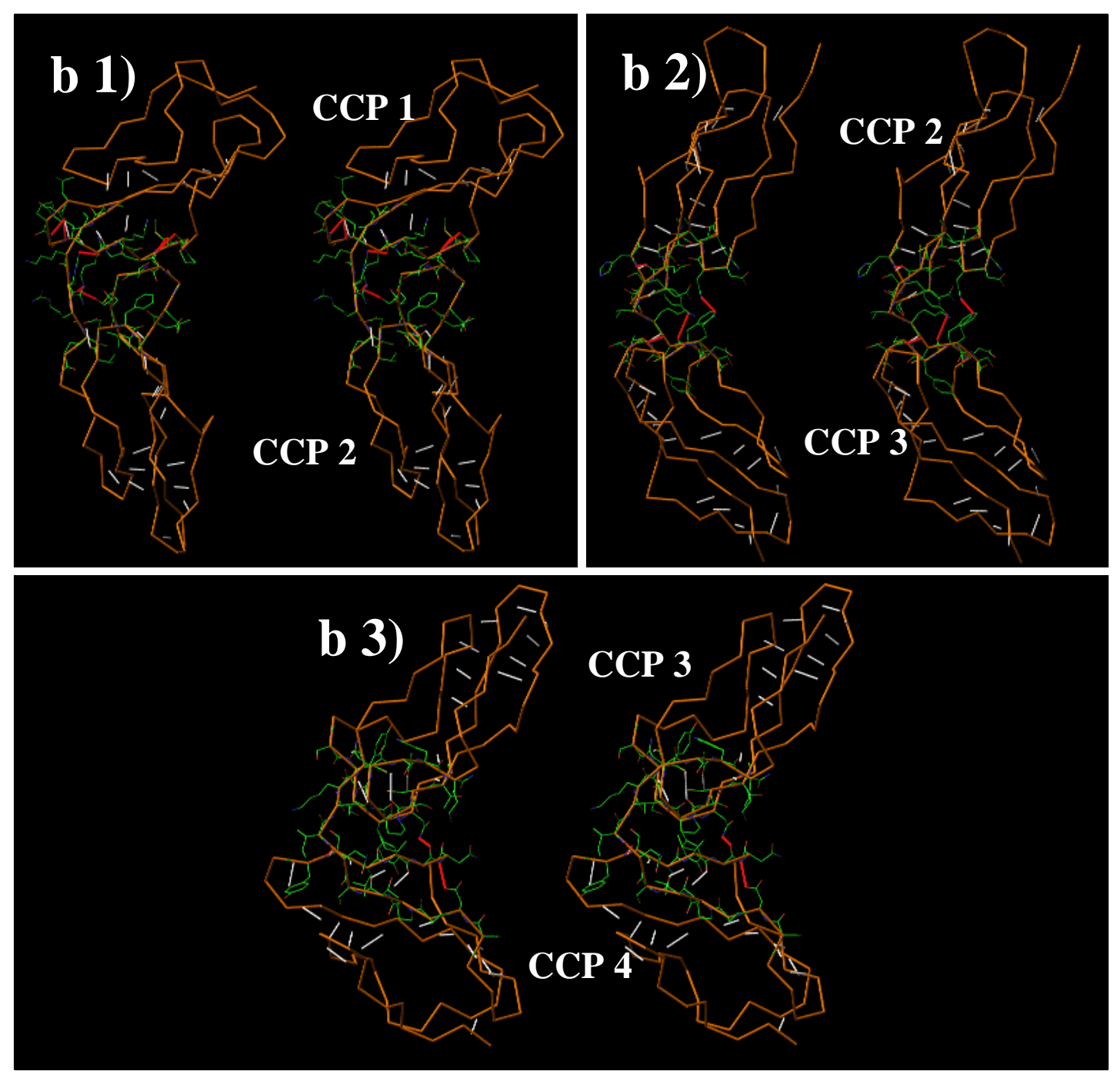


conserved $\mathrm{C}$ residues - the first $\mathrm{C}$ that is also the first residue of the $\mathrm{CCP}$ consensus sequence is bonded to the third $\mathrm{C}$, while the second $\mathrm{C}$ is bonded to the fourth $\mathrm{C}$ that is the last residue of the consensus sequence - see figure 57 for disulphide bonding pattern. The two disulphides lie as far apart as possible within the structure and form the lower and upper boundaries of the hydrophobic core. It is likely these disulphides survive elevated temperatures and their presence probably explains the reversibility of denaturation. The core almost entirely buries the side-chain of the invariant $\mathrm{W}$ residue and contains a number of highly conserved aromatic and L, I and V side-chains. Thus, each module consists of a compact hydrophobic core, bounded by two disulphides and wrapped in $\beta$-sheet. In the crystal structure (and in the average NMR structures), the CCP modules of rVCP are arranged such that the eight disulphides form a twisted ladder with roughly parallel and evenly spaced rungs.

From the crystal structure, we know that within rVCP, modules 1 and 2, and (particularly) modules 3 and 4, touch against one another. The 1-2 junction within the crystal structure exhibits many intermodular H-bonds and this could explain why module 2 is more stable (higher $\mathrm{T}_{\mathrm{m}}$ ) when module 1 is present (comparing the calorimetric profiles obtained for $\mathrm{rVCP}$ and for $\mathrm{rVCP} 2,3$ ) - see figure 56b. A similar effect is seen amongst the CCP modules of CR1 (Kirkitadze et al., 1999). In the 3-4 junction, as seen in both X-ray and NMR (Henderson et al., 2001) structures, modules make mainly hydrophobic contacts with one another and these two modules are tilted to create a significant interaction area. Module 3 may be more stable in full length rVCP than in $\mathrm{rVCP} 2,3$ but it is also possible that the melting of module 3 accounts for the first transition in VCP's calorimetric profile $\left(\mathrm{Tm}=58^{\circ} \mathrm{C}\right)$ which compares well to the $\mathrm{Tm}$ 
Figure 57. Disulphide bonding pattern within CCP modules of VCP. Sequence of the four $\mathrm{CCP}$ modules of $\mathrm{VCP}$, following signal sequence cleavage, is shown. Invariant $\mathrm{W}$ residue is shown in blue. The highly conserved $\mathrm{C}$ residues are shown in red. Disulphide bonding between CCP 1 (C21-C70 and C54-C81) CCP 2 (C86-C126 and C112-C143) CCP 3 (C148-190 and C176-201) CCP 4 (C206-248 and C234-261) are designated with brackets. 
CCP 1 CCTIPSRPINMKFKNSVETDANANYNIGDTIEYLCLPGYRKOKMGPIYAKCTGTGWTLFNOCI

CCP 1 CCTIPSRPINMKFKNSVETDANANYNIGDTIEYLCLPGYRKQKMGPIYAKCTGTGWTLFNQCI

CCP 2 KRRCPSPRDIDNGQLDIGGVDFGSSITYSCNSGYHLIGESKSYCELGSTGSMVWNPEAPICE

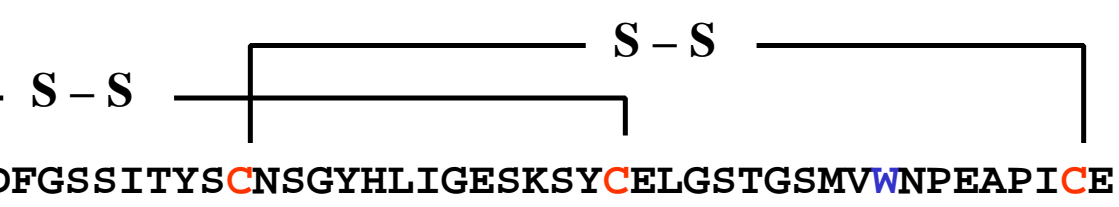

Г $\mathrm{S}-\mathrm{S} \longrightarrow \mathrm{S}-\mathrm{S} \longrightarrow$

CCP 3 sVKCQSPPSISNGRHNGYEDFYTDGSVVTYSCNSGYSLIGNSGVLCSGGEWSDPPTCQ

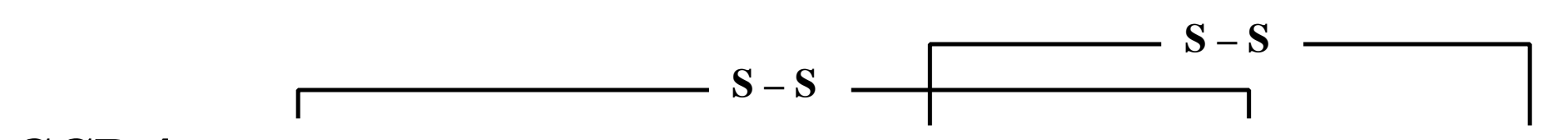

CCP 4 IVKCPHPTISNGYLSSGFKRSYSYNDNVDFKCKYGYKLSGSSSSTCSPGNTWKPELPKCVR 
$\left(57^{\circ} \mathrm{C}\right)$ of $\mathrm{rVCP} 2,3$. The $2-3$ junction itself is the smallest one and NMR studies indicate that this is a point of flexibility within the protein. Thus it is likely that module 2 derives little or no stabilization from module 3 and this would account for its poor thermal stability in $\mathrm{rVCP} 2$,3. A previous analysis of NMR relaxation times (Henderson et al., 2001) showed the backbone of module 3 to be more flexible than module 2 despite module 3 undergoing melting at a higher temperature - so it is possible that some of the stability of CCPs also derives from "give" in the structure that allows for local motion without denaturation.

Overall, VCP is a protein that, unusually, combines three properties. First, it is relatively small in terms of number of amino acid residues (and therefore is an economically encoded protein in terms of the limited size of the viral genome). Second, its highly elongated shape creates a large surface area that is available for binding to $\mathrm{C} 3 \mathrm{~b}$, C4b and proteoglycans. Lastly, as shown in the current study, it is both stable and robust, able to tolerate a range of adverse circumstances.

The consensus sequence that defines CCP modules is found several thousand times in the database (normally in tandem with other CCPs - solitary CCPs are rare). In comparison to other module-types, there is an unusual degree of variation in the spacing between the four consensus $\mathrm{C}$ residues, and in sequence apart from the consensus residues themselves. This indicates that the CCP module consensus sequence provides a highly versatile framework, tolerant of many insertions, deletions and substitutions, and it is consistent with the observations recorded here showing the CCP scaffold to be robust. Knowledge obtained from this study may be valuable in understanding the stability of other CCPs. It would also be interesting to know if other non-CCP members of this large 
SCR containing family of proteins also possess the unusual ability to retain functional activity after being subjected to extreme physical conditions. The question of whether VCP is stable having been answered, the next question is why? One of the hallmarks of the complement components is their heat stability up to $56^{\circ} \mathrm{C}$. Our study indicates that rVCP is at least as stable as the complement components. It is therefore possible that their co-existence over an extensive evolutionary period has resulted in this comparable stability. The importance of the disulfide bonds, for both stability and specific activity, is currently being investigated.

Due to the many extraordinary activities exhibited by this protein, its use as a novel immunomodulating agent is currently being investigated for treatment of many complement-mediated diseases. Determining the stability of rVCP was crucial for shipping, storage, administration, and dosage purposes as well as future proteinengineering efforts. rVCP has therapeutic potential as a means of ameliorating the complement-mediated damage associated with many medical conditions. The findings presented in this study may be important for future use of VCP in the treatment of many complement mediated diseases, such as: Alzheimer's disease, restonosis, systemic lupus erythematosus, and xenograft transplant rejections. 


\section{CHAPTER 6}

\section{PHARMACOKINETICS OF VCP}

\section{$\underline{\text { Introduction }}$}

This chapter contains material adapted from the following manuscript:

Anderson J.B., Smith S.A., Wijk R.V., Chien S., and Kotwal G.J. Vaccinia virus complement control protein inhibits hyperacute xenorejection in a guinea pig-to-rat heterotopic cervical cardiac xenograft model by blocking both xenoantibody binding and complement pathway activation. Submitted to Transpl. Immunol., 2002.

Simply stated, pharmacokinetics is the study of the process by which a drug is absorbed, distributed, metabolized, and eliminated by the body. Many routes of drug administration exist, including intravenous, oral, buccal, sublingual, rectal, intramuscular, transdermal, subcutaneous, inhalational, and topical. Ultimately, the goal of any pharmacokinetic study is to develop a means to maintain a steady-state concentration of the drug with rate of elimination equal to rate of administration, and the dosing rate must equal the clearance rate times the desired plasma concentration (Carson and Jones, 1979). Until recently, the use of proteins as potential therapeutic agents had not received much attention. However, due to the recent advances in protein purification and recombinant DNA techniques, pharmacokinetic studies of proteins are becoming much more common. The most common, by far, mode of administration used in these studies is intravenous. Unfortunately, proteins are often cleared from the blood very rapidly. This occurs most commonly via glomerular filtration in the kidneys, and the protein is excreted in the 
urine. The tendency for a protein to be eliminated from circulation by glomerular filtration depends mainly on two factors, the size of the protein and its electrostatic charge. Small proteins, less than $40 \mathrm{kDa}$, are often removed very rapidly. Likewise, the glomerular barrier also discriminates according to a protein's charge (Brenner et al., 1978). Negative charge seems to have the effect of reducing clearance of a protein via filtration. Therefore, in order to increase the half-life of a therapeutic protein in the blood, one must increase its size and/or negative charge. Many strategies have been developed to accomplish this, including: addition of polyethylene glycol (PEG) chains (PEGylation), covalent cross-linking, immunoglobulin domain fusion, and covalent fusion with albumin.

Most of the therapeutic studies that have been published in the area of CCPs involve the use of soluble complement receptor one (sCR1). sCR1 has been studied for its therapeutic use in, among other things, graft rejection, arthritis, allergic encephalomyelitis, and antigen-induced bronchoconstriction (Pratt et al., 1997; Mizuno et al., 2000; Piddlesden et al., 1994; Regal et al., 1993). These studies used different dosages and different routes of delivery. Early studies indicated that the effective intraperitoneal dose, as determined by the serum CH50, was $20 \mathrm{mg} / \mathrm{kg} /$ day (Piddlesden $e t$ al., 1994). In addition, the plasma sCR1 concentration was found to remain relatively constant from day to day. These results indicate that this dosage would result in a complement $\mathrm{CH} 50$ hemolytic activity below 5 for at least 6 days. For the transplantation studies using sCR1, an intravenous dosage of $25 \mathrm{mg} / \mathrm{kg} /$ day was used throughout the experimental time course (Pratt et al., 1996). Studies testing the ability of sCR1 to reduce the severity of experimentally induced arthritis found that, in addition to a direct 
intraarticular dose, an intravenous dose of $15 \mathrm{mg} / \mathrm{kg} / 12 \mathrm{~h}$ was very effective (Goodfellow et al., 2000). Taken together, it would seem that the half-life of sCR1 is relatively long; glomerular filtration of sCR1, therefore, would appear to be low. This is most likely due to the protein's size; possessing 20 SCRs (60-70 amino acids each), it is much too large to be easily excreted in the urine.

Several other CCPs have been used as therapeutic agents. Soluble versions of CD59 (sCD59), DAF (sDAF), and MCP (sMCP) have been investigated for use in treatment of complement-mediated disease. In addition, various chimeric fusion proteins have been created in an attempt to lengthen the serum half-life of a complement inhibiting protein. One such experiment used a chimeric protein created by fusing the active domains of MCP with a recombinant soluble form of the immunoglobulin receptor, Fc $\gamma \mathrm{RII}$ (Lanteri et al., 2000). Results indicated that this chimera possessed a much greater serum half-life than either of its components acting alone. Moreover, when less than $0.5 \mathrm{mg}$ of the protein was injected intravenously, it was found to block hyperacute rejection in a mouse (wt) to mouse (Gal o/o) heterotopic xenograft model.

Until this time, there has been no data determining the pharmacokinetics of VCP in vivo. Knowing the size and the charge of $\mathrm{VCP}$, one might predict that it would very easily be filtered by the kidneys, eliminated rapidly from the blood, and excreted in the urine. Initial in vivo experiments utilized FITC-labeled VCP injected intravenously to attempt to determine its localization. As expected, VCP was found to localize along the endothelium, near to the site of injection, most likely binding to heparan sulfate present on the endothelial cell surfaces. However, the results of this experiment did not demonstrate the intense staining that was anticipated. The cause of this was later 
revealed when FITC rVCP was found to bind heparin much less effectively than unlabeled rVCP - see figure 16. It seems clear that the label or the labeling process results in a dramatic reduction in VCP's ability to bind strongly to heparin, possibly by masking the binding sites or preventing proper folding and alignment of the sites. Using unlabeled rVCP and staining with anti-rVCP and labeled secondary antibodies solved this problem.

Single bolus injection studies were then conducted on live anesthetized rats. Various dosages and delivery locations were attempted while the pharmacokinetics was monitored by periodic collection of serum, for $\mathrm{CH} 50$ analysis, and urine, for $\mathrm{CH} 50$ and SDS-PAGE analysis. In addition, to understand how delivery could be optimized for use in long-term experiments, multiple or continuous injection strategies would be required. Therefore, various multiple and continuous intravenous dosages were attempted while serum was periodically collected for $\mathrm{CH} 50$ analysis. Finally, in an attempt to increase the serum half-life of rVCP, various strategies of protein modification were attempted. Efforts were made to covalently oligomerize rVCP, and to covalently link it to albumin, thus, in each case making the protein larger and less likely to be eliminated by glomerular filtration. Unfortunately, both attempts failed, either resulting in an inactive modified protein, or an inadequate amount of dimerized rVCP. 


\section{Materials and Methods}

\section{Single Injection Strategies:}

Pharmacokinetic studies were done with the surgical help of Jaime Anderson. In order to insure that the desired quantity of rVCP was entering the bloodstream, rVCP was injected directly into the jugular vein of anesthetized rats (anesthetized using $55 \mathrm{mg} / \mathrm{kg}$, I.P., sodium pentobarbital). A small incision was made in the neck of each rat, and the dose of rVCP was injected directly into the jugular vein making sure no leakage resulted. The small lesion was then closed with superglue and the animal was allowed to awaken. Blood was taken before the injection and periodically afterwards by cutting off the extreme tip ( $2 \mathrm{~mm})$ of the tail using a fresh scalpel blade. After the experiment was complete, the rats made a full recovery and were used in other unrelated experiments.

\section{Multiple and Continuous Injection Strategies:}

Several methods were attempted in order to administer rVCP over an extended time period. The first was to use an external syringe pump and inject rVCP into the jugular vein while the rat remained anesthetized. This method, using a Harvard Apparatus 22 syringe pump, was not practical due to the length of time the rat needed to be anesthetized. The second method that was attempted used an implantable rodent pump. This Harvard Apparatus implantable infusion pump was powered by an elastomer spring and delivered a constant rate of drug delivery. The pump was implanted subcutaneously in the rat's back, with the line running subcutaneously to the jugular vein. Although the pump allowed the rats to awaken while being studied, because this pump supplied a steady flow rate of only $0.12 \mathrm{ml} /$ day, it was also unpractical. In order to 
deliver the amount of rVCP needed to maintain the desired level in the blood, the protein concentration was much too high and resulted in a clogged line. Finally, an intermittent strategy of administration was attempted. A small I.V. port was superglued externally to the back of the rat, while the line ran subcutaneously to the jugular vein. With the rat awake, any amount of rVCP could very easily be injected as often as desired. This strategy was most effective, but required continuous attention.

\section{CH50 Hemolytic Activity Assay:}

To determine the complement activity of a serum sample, the complement hemolytic activity at 50\% (CH50) assay was employed. This assay greatly resembles the hemolysis assay, however it tests the serum complement activity as opposed to testing the ability of a protein to inhibit serum complement. Both assays use sSRBCs to accurately quantify complement activity, or the inhibition thereof. The $\mathrm{CH} 50$ assay determines the serum complement activity by identifying the quantity of a given serum needed to lyse $50 \%$ of the sSRBCs in the assay. Therefore, serially diluted serum samples were incubated with a known concentration of sSRBCs, and the \% lyses was determined by comparing the sample to a positive control, usually water lysed sSRBCs.

\section{Detection of rVCP in Urine:}

Urine samples were collected by a $25 \mathrm{G}$ urinary catheter before and after rVCP injection. All samples were then concentrated and dialyzed against water to the same final volume. First, the urine sample was centrifuged at $10,000 \mathrm{rpm}$, in order to pellet contaminating bacteria and epithelial cells. Next, each sample was filtered through a 0.2 
$\mu \mathrm{m}$ syringe filter. The samples were then placed in separate centricon concentrators and centrifuged at 7,000 rpm at $4^{\circ} \mathrm{C}$. After the samples were concentrated to the same volume $(\sim 100 \mu \mathrm{l})$, they were dialyzed by addition of $2 \mathrm{ml}$ sterile water. Finally, the urine samples were analyzed by hemolysis assay and visualized by SDS-PAGE and Coomassie blue staining.

\section{Oligomerization and Modification of rVCP:}

In order to couple rVCP to BSA or to itself, standard amine coupling procedures were used. To couple rVCP to BSA, $5 \mathrm{mg}$ of BSA was suspended in $5 \mathrm{ml}$ activation buffer: 100 mM MES, 500 mM NaCl, pH 6.0. Next, 2 mg of N-ethyl-N'(dimethylaminopropyl)-carbodiimine (EDC) and $3 \mathrm{mg}$ of N-hydroxysuccinimide (NHS) were added to the solution. The solution was then allowed to incubate at room temperature for $15 \mathrm{~min}$. In order to quench the EDC, $7 \mu \mathrm{l}$ of $\beta$-mercaptoethanol was added to the mixture. Finally, $4 \mathrm{mg}$ of $\mathrm{rVCP}$ was added to the solution and allowed to incubate at room temperature for 2 hours. After the incubation period, $0.4 \mathrm{~g}$ of hydroxylamine was added to reform the uncoupled carboxyl groups on the BSA. The mixture was then concentrated and dialyzed to eliminate all remaining chemicals. The same protocol was used to form rVCP dimers. 


\section{$\underline{\text { Results }}$}

\section{Single Bolus Injection Studies:}

In order to determine the half-life of rVCP in the serum of rats, various routes of admission and dosage strategies were investigated. Starting with a relatively high dose of $3 \mathrm{mg}(0.5 \mathrm{ml}$ total bolus), preliminary intravenous (I.V.) and intraperitoneal (I.P.) injections were attempted while the serum $\mathrm{CH} 50$ was monitored. As can be seem in figure 58a, clear complement depletion is observed by $15 \mathrm{~min}$ following I.V. injection of rVCP. This activity rapidly starts to decrease and the serum complement approaches normal levels. This decrease (lack of complement activity) is not observed when rVCP is injected into the peritoneum of the rat - see figure 58b. Only a very small amount of the protein makes it to the serum and is detected. The inability of rVCP to enter the blood from the tissue was also observed when a rat was injected subcutaneously with a large 24 $\mathrm{mg} / \mathrm{kg}$ bolus. Very little serum complement depletion is observed by the $\mathrm{CH} 50$ of this rat. The results of the above experiments determined that rVCP must be injected intravenously to work most effectively. Therefore, in order to establish the most effective I.V. dose, rats were injected with various bolus amounts of rVCP. The averaged results (2 rats each) of a single dose of $3 \mathrm{mg} / \mathrm{kg}, 6 \mathrm{mg} / \mathrm{kg}$, or $12 \mathrm{mg} / \mathrm{kg}$ are shown in figure 59. A clear difference can be seen between the slopes of the $\mathrm{CH} 50$ s for the various doses. Thus, the rate of clearance can be effectively predicted from this data. See the appendix for figures showing additional preliminary results of single animal studies.

The ability of rVCP to deplete serum complement was also investigated in baboons. Due to the extreme size of this animal, large quantities of the protein must be made and purified. Therefore, the kinetics of rVCP in baboons must be worked out using 
Figure 58. CH50 following I.V. and I.P. injection of $3 \mathrm{mg}$ rVCP. A) Complement depletion in rat injected intravenously with $3 \mathrm{mg}$ rVCP. Serum complement activity at $15 \mathrm{~min}, 30 \mathrm{~min}$, and $60 \mathrm{~min}$ is shown. Significant depletion of complement activity can be observed. B) Complement depletion in rat injected intraperitoneally with $3 \mathrm{mg} \mathrm{rVCP}$. Serum complement activity at $15 \mathrm{~min}, 30 \mathrm{~min}, 60 \mathrm{~min}$, and 12 hours is shown. No depletion of complement activity is evident. 


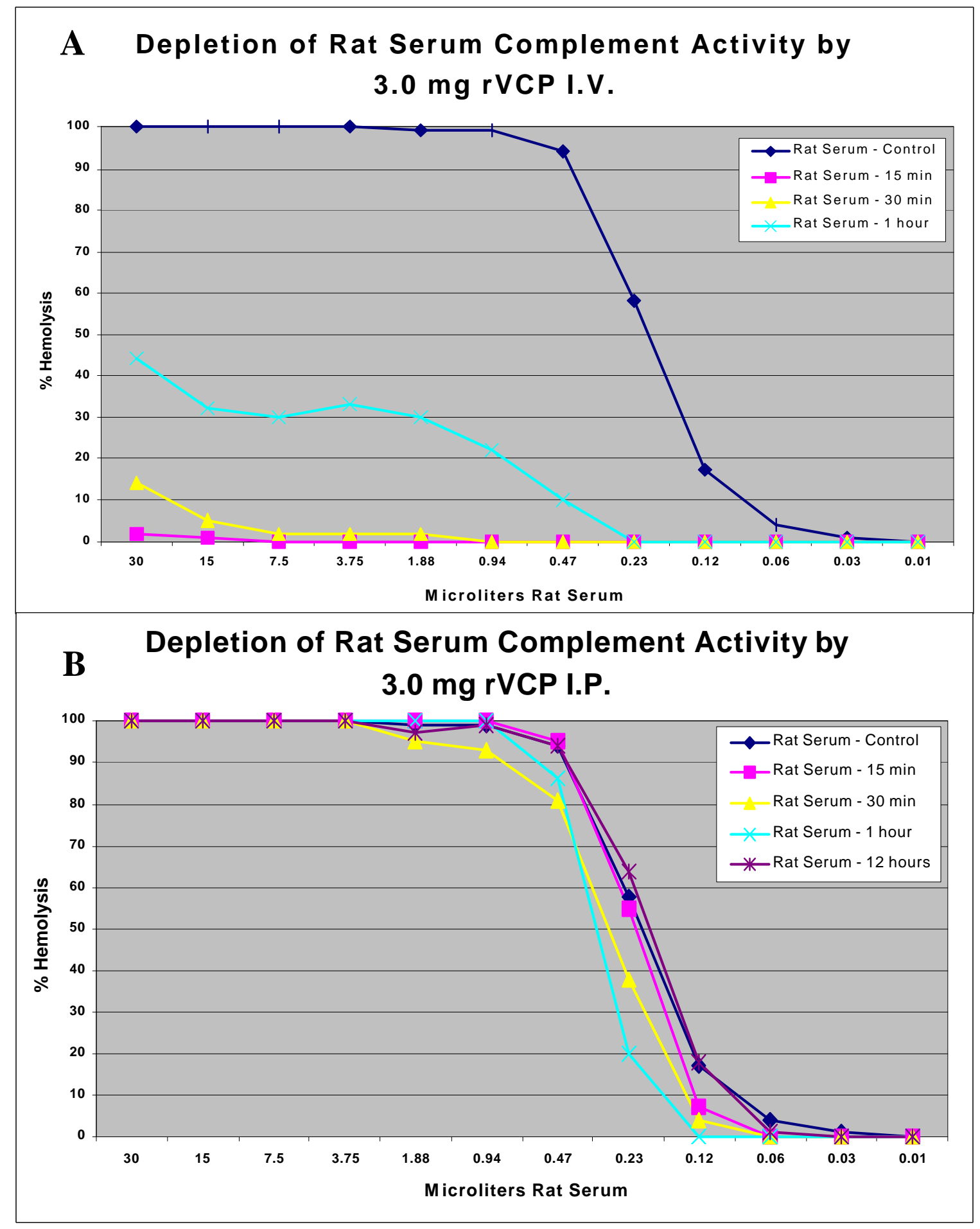


Figure 59. Serum CH50 of I.V. injected rVCP at 3, 6 and $12 \mathrm{mg} / \mathrm{kg}$. Two rats in each group were administered either 3,6 or $12 \mathrm{mg} / \mathrm{kg} \mathrm{IV}$ of $\mathrm{rVCP}$ and the serum was collected at 15 minutes intervals and assayed for $\mathrm{CH} 50$ as compared to serum control prior to rVCP administration. The pharmacokinetics of $\mathrm{rVCP}$ in rats can be accurately deduced by this data. 


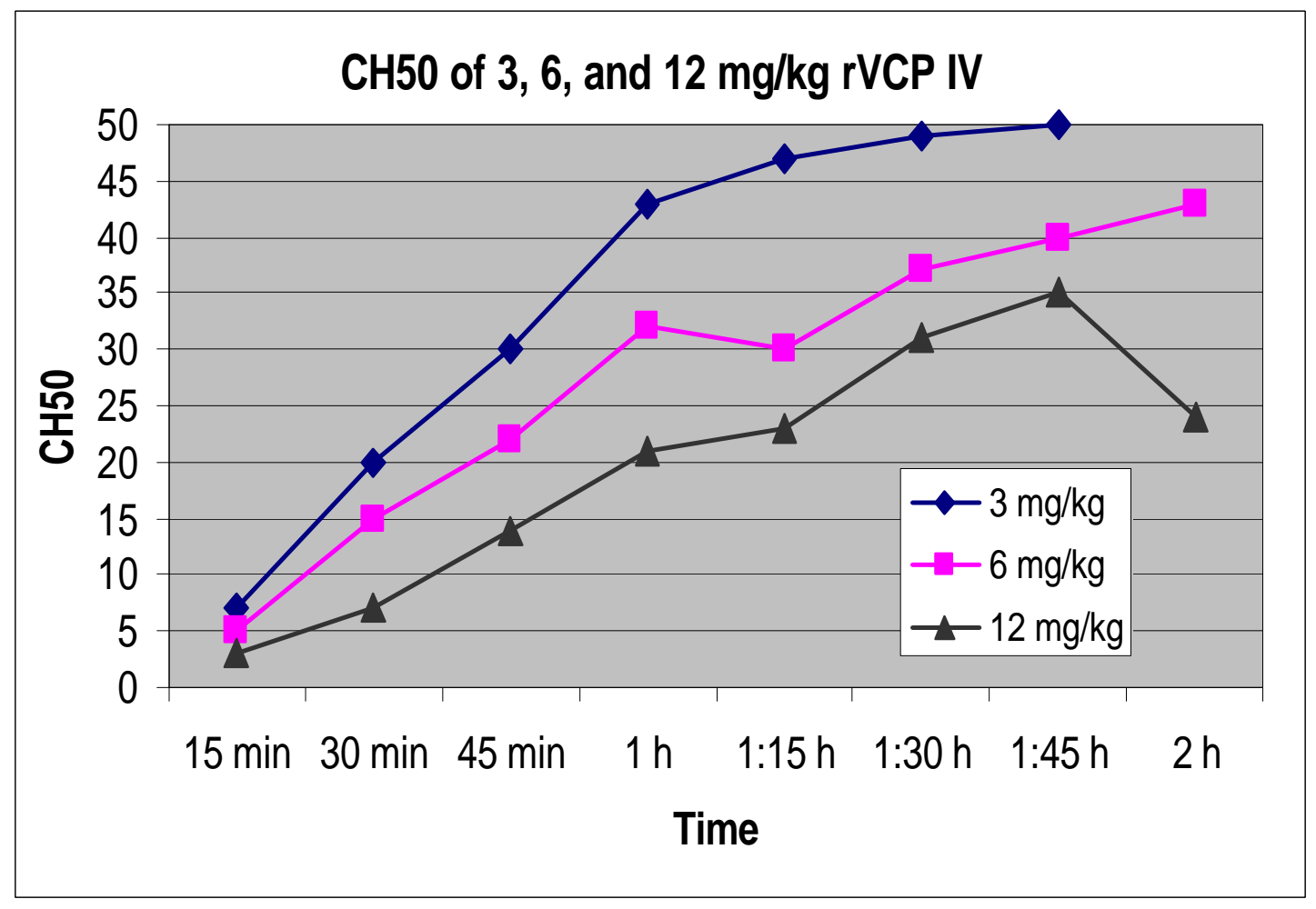


only a few experimental animals and by comparing their data with that obtained in the rat studies. The first baboon in the study (University of Cape Town, Cape Town, SA) was injected intravenously with $45 \mathrm{mg}$ rVCP bolus and the serum and urine were collected for analysis. Due to the extreme size and the small amount of rVCP that was injected $(\sim 3$ $\mathrm{mg} / \mathrm{kg}$ ), no complement activity depletion was observed at any time point, even $15 \mathrm{~min}$. The size of the I.V. dose was then increased to $9 \mathrm{mg} / \mathrm{kg}$ in hopes of seeing even a slight decrease in CH50. Baboon 479 (15.8 kg) showed a slight drop in serum CH50 at the 15 min time point. The $\mathrm{CH} 50$ was reduced to 37 (37\% lysis using the pre-treatment serum dilution that resulted in $50 \%$ lysis) by $15 \mathrm{~min}$, but returned to normal by the next time point, $30 \mathrm{~min}$. An additional baboon was injected with the same dose, $9 \mathrm{mg} / \mathrm{kg}$, but was injected using an I.V. drip. Baboon $397(17.1 \mathrm{~kg})$ received rVCP over 15 minutes in an I.V. drip, as opposed to baboon 479 who got a single bolus. As can be seen in figure 60, the serum $\mathrm{CH} 50$ of baboon 397 was much higher than that observed in baboon 479 at the 5 min time point. Baboon 397 had a $\mathrm{CH} 50$ of 30, while baboon 479 had a $\mathrm{CH} 50$ of only 5 , after $5 \mathrm{~min}$. This does not suggest that the single bolus injection strategy of administration was more effective in depletion of serum complement activity, because the drip was not completed until the 15 min time point. More animals would have to be tested in order to confirm these results. However, the data obtained by these experiments may be sufficient for determining the approximate clearance rate of $\mathrm{rVCP}$ in the baboon. See the appendix for figures showing additional preliminary results of single animal studies. 
Figure 60. Baboon 479 and 397 CH50 following 9 mg/kg I.V. rVCP. Serum samples taken from baboon 397, which received rVCP in an I.V. drip, and 479, which received rVCP as a bolus, were tested for complement activity with the CH50 assay. The CH50 was tested at time 0 and 5 min following completion of the injection. The verticle lines represent the CH50s for pre-treatment sera. 


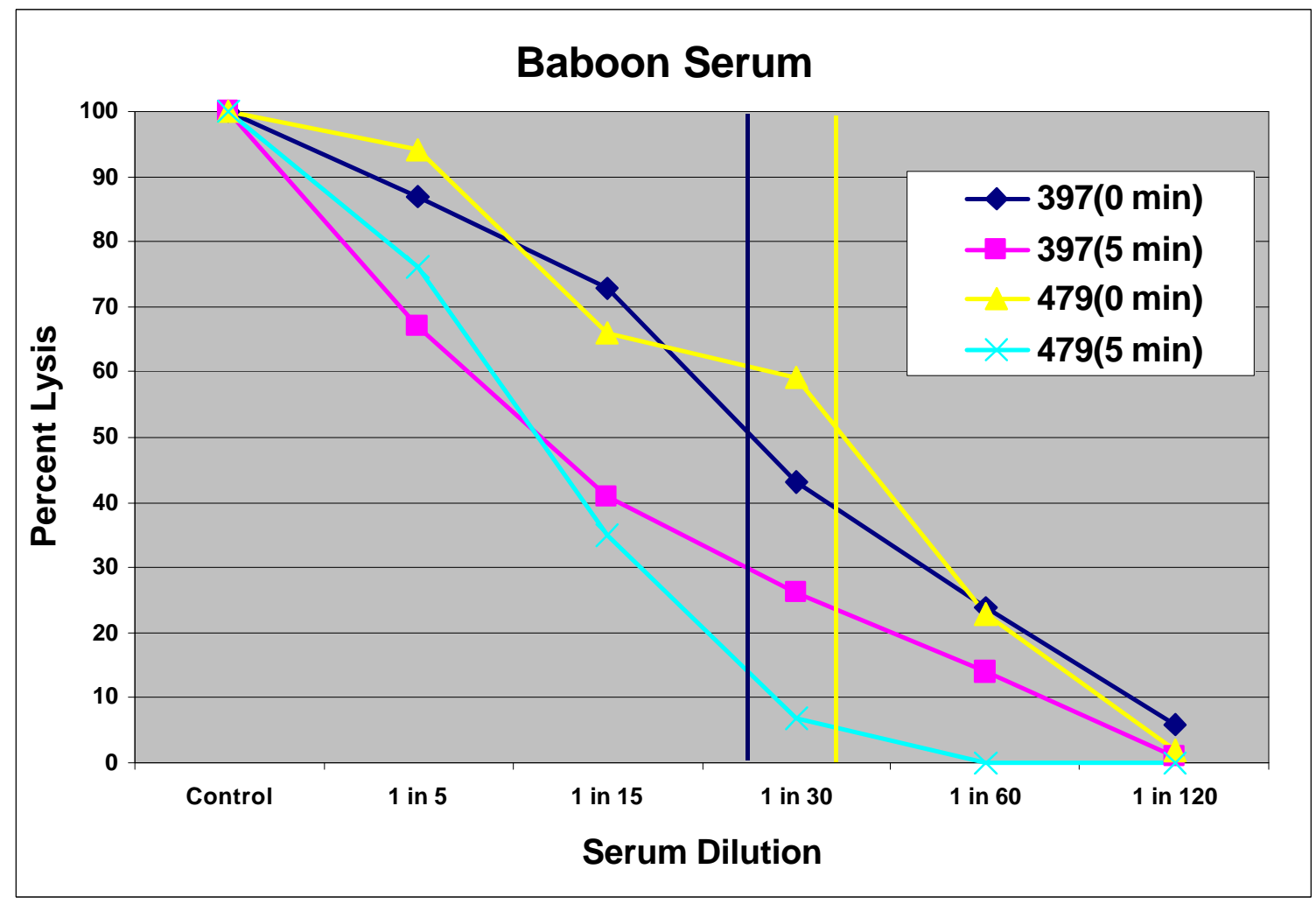




\section{Multiple and Continuous Injection Studies:}

To further exploit rVCP's therapeutic potential, it would have to be administered over an extended period of time. Therefore, preliminary attempts were made to inject rVCP I.V. continuously or intermittently for an extended time. The initial experiments were attempted using an external syringe pump. With the rat sedated, $\mathrm{rVCP}$ was pumped into the jugular vein at a continuous controlled rate, $16 \mathrm{mg} / \mathrm{kg} / \mathrm{hr}$ was injected and blood samples were taken 15, 30, and 60 min. This method worked well, controlling the complement activity by maintaining the $\mathrm{CH} 50$ below 10 . Next, an implantable pump was used in order to allow the rat to awaken. With the pump implanted subcutaneously in the back of the rat, the small injection line was sutured to the jugular vein. The type of pump used had a fixed rate of drug administration. With the injection rate of the pump in mind, rVCP was added to the pump such that a steady concentration of rVCP would be present in the blood. First, rVCP was added to the pump such that a continuous dose of 3 $\mathrm{mg} / \mathrm{kg} / \mathrm{hr}$ would be given, following an initial bolus of $6 \mathrm{mg} / \mathrm{kg}$. Complement activity was initially depleted but very slowly increased over the 6-hour time course. Although the CH50 never returned to normal levels, this demonstrates that an insufficient amount of rVCP was being injected. Therefore, a higher dose was attempted, trying a concentration of rVCP high enough to deliver $6 \mathrm{mg} / \mathrm{kg} / \mathrm{hr}$. Unfortunately, this concentration was too high and resulted in a clogged pump line. Thus, less rVCP was detected in the serum and resulted in a more rapid return of complement activity, as detected by the increase in CH50 to near normal levels. Because of these experiments, the continuous method of injection, via an implantable pump, has been set aside. A different strategy for long-term delivery had to be worked out in order to administer large 
amounts of rVCP continuously. See the appendix for figures showing preliminary results of single animal studies.

To deliver rVCP over extended periods of time without the aid of an implantable pump, a catheter technique was developed. A small I.V. catheter port was fixed to the back of the rat by superglue, and a small injection line was run subcutaneously and sutured to the jugular vein. After an initial $6 \mathrm{mg} / \mathrm{kg}$ bolus was given, $2 \mathrm{mg} / \mathrm{kg}$ was injected through the I.V. catheter every $20 \mathrm{~min}$ to sustain a concentration of $6 \mathrm{mg} / \mathrm{kg} / \mathrm{hr}$. The complement activity was depleted to below a $\mathrm{CH} 50$ of 5 after $15 \mathrm{~min}$ and was held to the same level at 6 hours. This strategy of long-term injection works well, however, it is very time consuming and not very practical. See the appendix for figures showing preliminary results of single animal studies.

\section{Presence of $r V C P$ in Urine:}

In order to investigate the mannor by which rVCP is cleared from the blood, various urine samples were taken and studied. Early animal experiments were conducted using $\mathrm{rVCP}$ that was unknowingly contaminated with endotoxin, approximately 30,000 $\mathrm{EU} / \mathrm{ml}$. Urine samples obtained from the animals injected intravenously with this rVCP revealed extremely high levels of the protein excreted into the urine. As can be seen in figure 61a, large concentrations of $\mathrm{rVCP}$ can be seen in two samples taken from rats injected with rVCP (endotoxin contaminated), but cannot be seen in the sample taken before injection. In addition, figure 61a shows urine samples taken (taken over several hours following injection) from rats and a baboon that received rVCP that was endotoxin free $(<10 \mathrm{EU} / \mathrm{ml})$. There is no visible sign of $\mathrm{rVCP}$ in the rat samples or the baboon 
Figure 61. Presence of $\mathbf{r V C P}$ in urine. A) SDS-PAGE analysis of rat urine and a baboon urine samples taken before and after administration of rVCP, contaminated with endotoxin (lanes 1-4), or endotoxin free (lanes 5-8). rVCP marker can be seen in lanes 1 and 5. Lane 2 represents urine before $\mathrm{rVCP}$ was injected while lanes 3 and 4 represent urine samples taken over 2 hours from two different rats following rVCP administration. Again, lane 6 represents urine before $\mathrm{rVCP}$ was injected while lane 7 represents urine taken for 2 hours following rVCP administration. Lane 8 shows a baboon urine sample following rVCP administration. B) Hemolysis inhibiting activity of rat urine samples taken before and after (for 2 hours) $\mathrm{rVCP}$ injection. Also shown is the hemolysis inhibiting activity of baboon urine after injection of $\mathrm{rVCP}$. As can be seen, a significant increase in hemolysis inhibition occurs after rVCP is injected, due to its presence in the urine. 


\section{rVCP with Endotoxin}

A

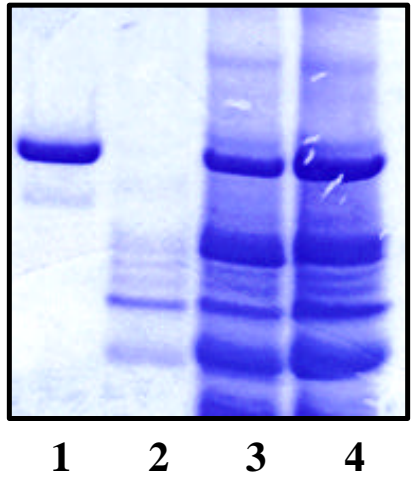

Endotoxin-free rVCP

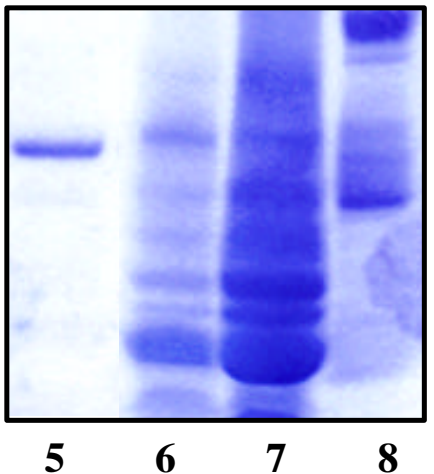

\section{B Hemolysis Inhibition by rVCP in Urine}

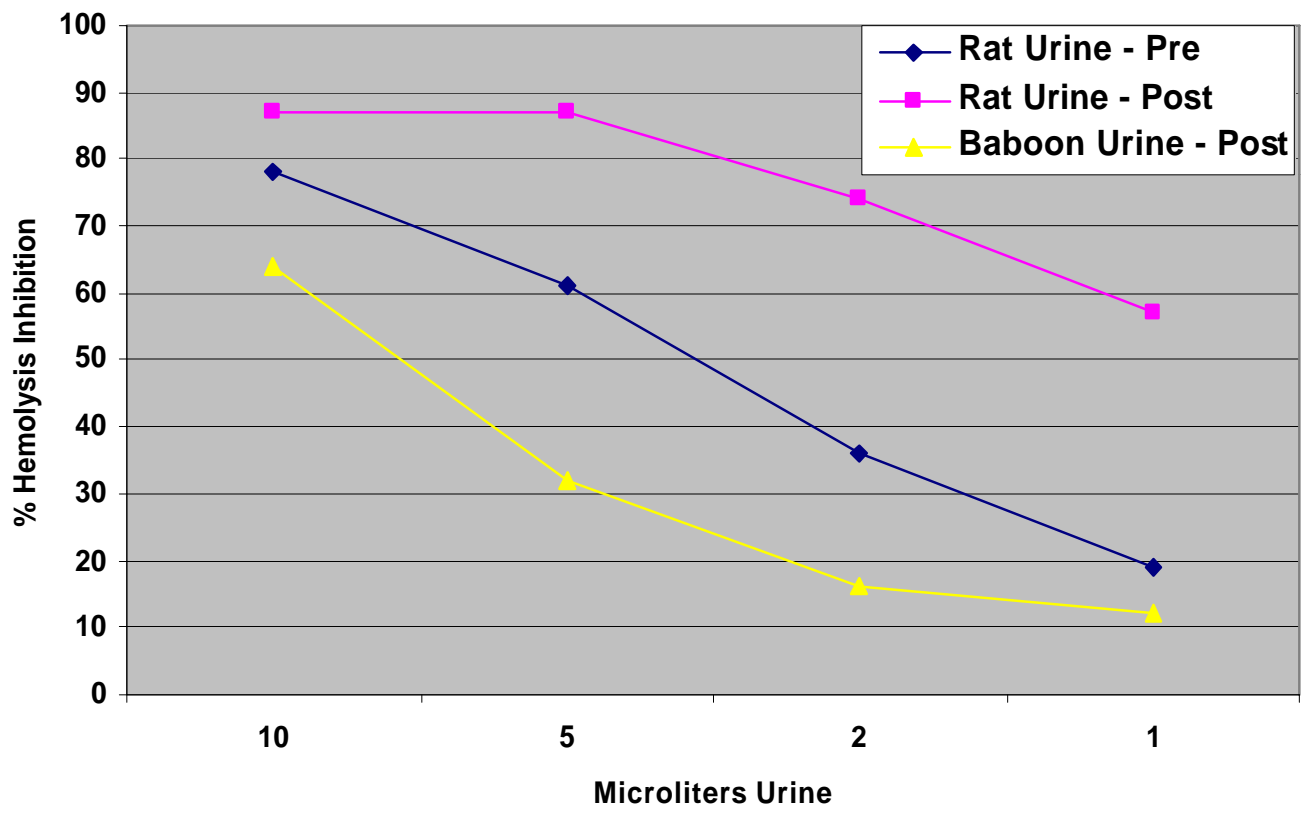


sample. Finally, the rat and baboon sample were tested for hemolysis inhibiting activity. Figure 61b shows that rat urine after rVCP (endotoxin free) was injected showed a significantly greater degree of hemolysis inhibiting activity. This can only be attributed to a small amount of active rVCP being present in the urine sample. The baboon urine sample showed no sign of rVCP by SDS-PAGE analysis or hemolysis assay.

\section{Attempts to Oligomerize and Modify rVCP:}

Due to the rapid rate at which rVCP is eliminated from the blood, most likely through glomerular filtration, attempts were made to extend the proteins half-life by modification or multimerization of rVCP. Using standard amine coupling procedures, rVCP was covalently conjugated to bovine serum albumin (BSA). As can be seen in figure 62 , rVCP coupled to BSA was purified away from uncoupled rVCP and uncoupled BSA by heparin affinity purification. Coupled rVCP-BSA bound heparin with less affinity than rVCP alone, allowing for complete separation from the unconjugated protein. Unfortunately, when rVCP-BSA was tested for hemolysis inhibiting activity it was found to be inactivated. Next, rVCP was coupled to itself by standard amine coupling procedures. As can be seen in figure 62, only a slight degree of dimerization took place. Not enough rVCP dimers were created to purify away from the uncoupled rVCP. Therefore, dimers of rVCP could not be tested for their ability to inhibit hemolysis in the hemolysis assay or if they could effectively increase the half-life of rVCP in the serum. 
Figure 62. Modification of rVCP using standard amine coupling. Heparin purification following the coupling of rVCP to BSA and rVCP to itself is shown by SDSPAGE analysis. Following rVCP coupling to BSA, heparin chromatography provides a way to purify out rVCP-BSA from both uncoupled rVCP and BSA. This is due to rVCPBSA having a lesser affinity for heparin than rVCP. However, rVCP coupled to itself can not be purified away from rVCP by this method. Thus, heparin chromatography is ineffective and gel filtration would be the most likely alternative. 

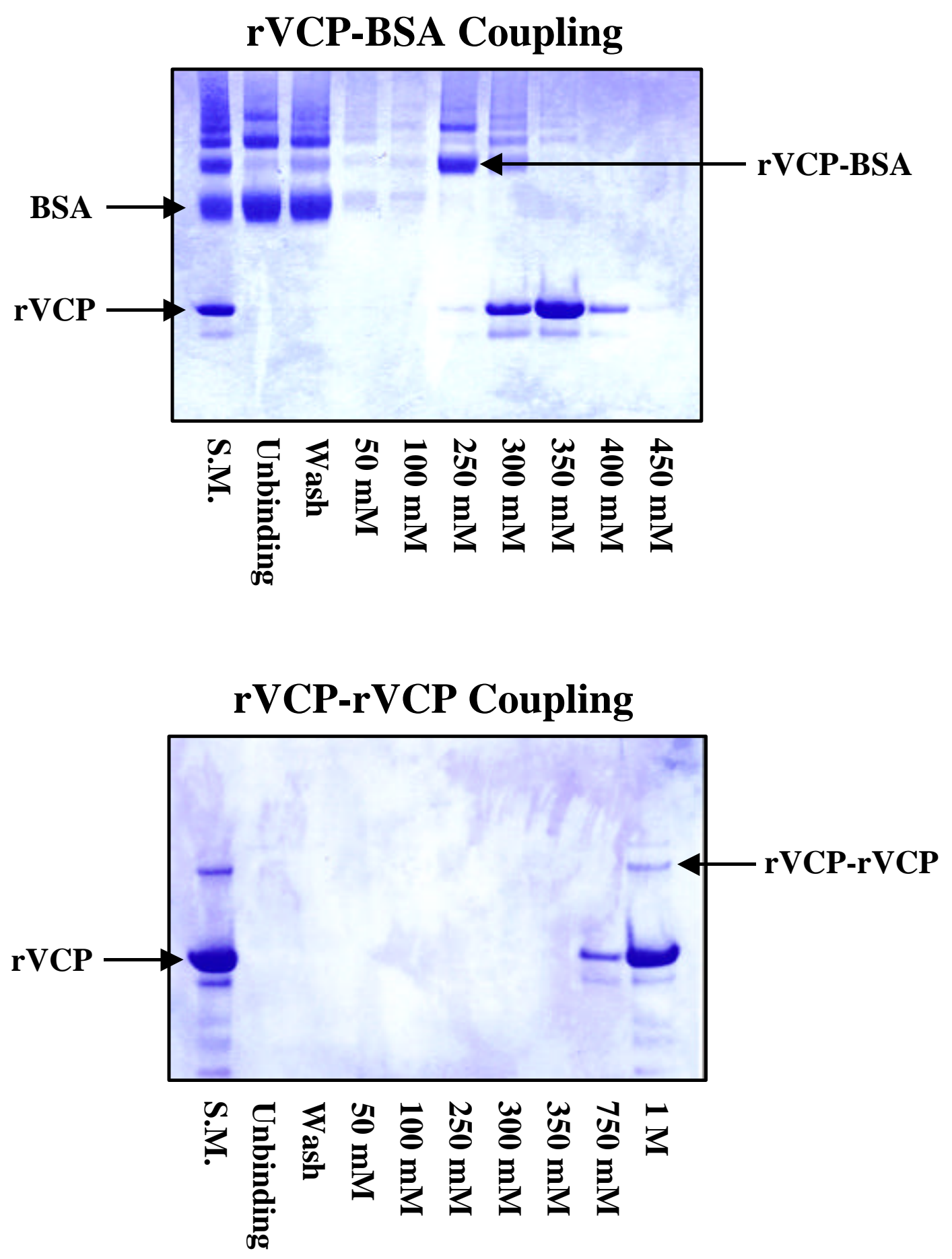


\section{$\underline{\text { Discussion }}$}

Initial experiments employed single bolus doses of rVCP delivered by various routes to try to determine the best dosage and location of delivery. It was determined that the intravenous (I.V.) route of injection was the most effective. Intraperitoneal and subcutaneous injection strategies were attempted with poor results. It is possible that the strong affinity that VCP possesses for heparin and heparan sulfate contributes to these findings. When $\mathrm{rVCP}$ is injected into these locations, it may bind to heparan sulfate proteoglycans on the surface of cells or to heparin containing mast cells. This could effectively prevent rVCP from entering the blood, even at a slow rate. Although nothing is known about what happens following rVCP binding to heparan sulfate on the surface of cells, studies are underway using electron microscopy. If the protein is internalized, and possibly degraded, it would result in the effect seen in the above experiments, where it is injected into the tissue and does not affect serum complement.

Using single bolus, continuous, and intermittent strategies of I.V. injection, the rate at which rVCP leaves the blood can be calculated relatively accurately. Using the data presented in figure 59 , for example, when $3 \mathrm{mg} / \mathrm{kg}$ is injected, the $\mathrm{CH} 50$ returns to around 20 at $30 \mathrm{~min}$ post injection. When $12 \mathrm{mg} / \mathrm{kg}$ is injected, it takes twice the amount of time before the $\mathrm{CH} 50$ reaches 20 . Therefore, it takes 4 times the amount of rVCP to double the time the protein effectively depletes serum complement activity. This data tells us a lot about the pharmacokinetics of rVCP. Obviously, rVCP is being eliminated from the blood at a very fast rate. By studying figure 59, it seems that half of the protein activity is eliminated, CH50 of 25, somewhere between 6 and $12 \mathrm{mg} / \mathrm{kg}$, closer to 6 $\mathrm{mg} / \mathrm{kg}$. Using this data one would predict that $\mathrm{rVCP}$ is eliminated from the blood at a 
rate of approximately $8 \mathrm{mg} / \mathrm{kg} / \mathrm{hr}$. Thus, $\mathrm{rVCP}$ would need to be administered continuously to be effective in sustaining a low serum $\mathrm{CH} 50$.

The studies where rVCP was injected I.V. continuously or intermittently also give us clues about the elimination rate of rVCP. Preliminary data shows that while the CH50 is immediately reduced by the $6 \mathrm{mg} / \mathrm{kg}$ bolus, it continues to rise despite a continuous infusion of $3 \mathrm{mg} / \mathrm{kg} \mathrm{rVCP}$. This demonstrates that $3 \mathrm{mg} / \mathrm{kg}$ is not sufficient for controlling complement levels in the blood. The study using a continuous infusion of 16 $\mathrm{mg} / \mathrm{kg} \mathrm{I.V}$. showed extremely reduced complement activity sustained for up to an hour. This dose was most likely "overkill" because of what was found when $6 \mathrm{mg} / \mathrm{kg}$ was injected intermittently following a $6 \mathrm{mg} / \mathrm{kg}$ bolus. Priliminary data shows that the serum CH50 was not only depleted at the early 15 min time point, but also at the latest time point of 6 hours. This study indicates that in order to maintain a $\mathrm{CH} 50$ below 5, one needs to continuously inject $6 \mathrm{mg} / \mathrm{kg} \mathrm{rVCP}$ intravenously. This supports the conclusions drawn by the bolus dose experiments, that rVCP is eliminated from the blood at a very high rate, somewhere between 6 and $12 \mathrm{mg} / \mathrm{kg} / \mathrm{hr}$. It also seems likely that the level of complement activity in the blood slowly decreases over time as rVCP is continuously administered. Therefore, it is quite possible that lower levels of rVCP would be needed at later time points and the dosage could be dropped.

The initial data collected from baboons injected intravenously with large bolus doses of rVCP shows that the protein is clearly not as effective as in rodents. This data supports conclusions drawn in chapter 4 , hypothesizing that $\mathrm{rVCP}$ is specific for the rat and therefore works most effectively against rat complement. Figure 60 shows the CH50 of a baboon injected with a $9 \mathrm{mg} / \mathrm{kg}$ bolus. After $15 \mathrm{~min}$, the $\mathrm{CH} 50$ was reduced to only 
37. The same dose if it were injected into a rat, would have eliminated complement activity, reducing the $\mathrm{CH} 50$ to 0 after $15 \mathrm{~min}$. It takes $45 \mathrm{~min}$ for the complement activity of a rat injected with only $3 \mathrm{mg} / \mathrm{kg}$ to reach $\mathrm{CH} 50$ of 37 . This clearly demonstrates the drastic in vivo difference in complement inhibiting activity possessed by rVCP between rat and baboon, and probably human. To achieve the same level of complement inhibition, one would have to inject approximately 5 times as much rVCP in a baboon as a rat. That means to sustain a $\mathrm{CH} 50$ of below 5 in a baboon, one would need to inject $30 \mathrm{mg} / \mathrm{kg} / \mathrm{hr}$ following a $30 \mathrm{mg} / \mathrm{kg}$ bolus.

To determine the means to which rVCP is eliminated from the blood and ultimately from the body, urine samples were collected and analyzed for traces of the protein. Knowing that the most likely mode of elimination of a protein of this size and charge is via glomerular filtration, large amounts of rVCP could likely be found in the urine. Early studies of rat urine revealed that very large concentrations of $\mathrm{rVCP}$ was present and could be clearly visualized by SDS-PAGE analysis. Although Western blotting would have more conclusively proven that this was in fact rVCP, the absence of the band in the urine before injection makes a strong case. These rats, however, received rVCP that possessed high concentrations of endotoxin. Interestingly, urine samples obtained from rats that received rVCP that contained no endotoxin showed very small traces of rVCP, as detected by hemolysis assay but not by SDS-PAGE analysis. The presence of the protein was determined, however, by testing the urine samples in the hemolysis assay. The urine sample after injection of rVCP possessed a significant ability to inhibit complement, when compared to a sample taken before rVCP was injected. This not only demonstrates the presence of rVCP in the urine, but also shows that at least 
some of the protein is eliminated in an active, fully functional form. It seems however, that the presence of endotoxin greatly increases the amount of rVCP being excreted in the urine. This may therefore result in a decreased half-life in the serum. Fortunately, this does not discredit the pharmacokinetic studies described above, because almost all of the studies were performed using aliquots of the same batch of protein $(16 \mathrm{mg} / \mathrm{kg}$ continuous infusion used a different batch that was not tested, figure 65 in appendix), which was tested and found to be nearly endotoxin free.

Due to the rapid elimination of rVCP from the blood, presumably via glomerular filtration, attempts were made to modify the protein to increase its serum half-life. Using standard amine coupling procedures, rVCP was covalently linked to BSA and to itself. Unfortunately, neither attempt proved fruitful. When rVCP was linked to BSA, it was easily purified, but possessed no activity and was thus useless. When rVCP was linked to itself, forming rVCP dimers in only low relative amounts, the protein resembled the monomeric protein too closely and could not be purified away from the uncoupled rVCP. In addition, the amount of rVCP dimers created was very low relative to the concentration of rVCP monomers. Theoretically, creating rVCP dimers should greatly increase the protein's half-life, by making the protein near to the size of albumin, if a method of purification could be devised, possibly using gel filtration chromatography. Despite these failed attempts at modifying rVCP, if rVCP were to be seriously considered for use as a therapeutic agent, efforts would have to be made to increase the protein's serum half-life. Other than gene therapy, which could theoretically deliver a constant dose of rVCP where it is needed, protein modification may be the best solution. As discussed in the introduction, many methods of protein modification have been employed 
to increase a proteins half-life in the serum. One effective method that was not investigated, although it has great potential, for use on rVCP, is PEGylation. This method of modification is growing in popularity due to its ability to not only result in a favorable size and charge, but also reduce the protein's antigenicity (Reddy, 2000; Mehvar, 2000). The procedure for PEG modification of proteins is relatively straightforward and has been described for many other proteins (Reddy et al., 2001; Wang et al., 2000; Hershfield et al., 1987). This procedure has significantly increased the half-life of many proteins, including several in clinical use, without affecting their activities, although, this does not necessarily mean it will work effectively for rVCP. 


\section{CHAPTER 7}

\section{FUTURE STUDIES OF VCP}

\section{$\underline{\text { Species Specificity Studies of rVCP and the Variola Virus Homologue }}$}

In order to investigate the species specificity of $\mathrm{rVCP}$ and the variola virus homologue of $\mathrm{VCP}$ using Biacore and in vitro hemolysis assays, the variola virus gene must be cloned from the viral DNA. Sergei N. Shchelkunov, a collaborator from the Department of Molecular Biology of Genomes, State Research Center of Virology and Biotechnology "Vector" Koltsovo, Novosibirsk Region, Russia, will PCR amplify the gene for cloning. The primers that will be used to amplify the variola homologue of VCP are: (sense) 5' GTA TCT CTC GAG AAA AGA TGC TGT ACT ATT CCG TCA 3' making a Xho I restriction site and (anti-sense) 5' TTA ATT CGC GGC CGC TTA GCG TAC ACA TTT TGG 3' making a Not I restriction site. Following gene amplification by PCR, the amplified product will be cloned into the Pichia pastoris yeast expression system. The same procedures and plasmids used to express rVCP (described in chapter 2) will be used for expression of variola virus VCP.

Once the variola virus homologue of VCP is in hand, the hemolysis assay will be

performed to determine whether this protein possesses greater activity against human and baboon complement than rVCP. The hemolysis assay will also be used to determine whether the variola virus homologue of VCP possesses less activity against rat and mouse complement than exhibited by rVCP. The Biacore will also be used to evaluate 
the strength of binding between $\mathrm{rVCP}$ and rat $\mathrm{C} 3 \mathrm{~b}$ or human $\mathrm{C} 3 \mathrm{~b}$ (purification and trypsinization described in chapter 4) and evaluate the strength of binding between the variola virus homologue of $\mathrm{VCP}$ and rat $\mathrm{C} 3 \mathrm{~b}$ or human $\mathrm{C} 3 \mathrm{~b}$. The results obtained using the hemolysis assay and Biacore binding analysis will determine whether these proteins interact with complement in a species-specific mannor.

It has already been shown that rVCP is much more effective in blocking rat/mouse complement than human/baboon, using the hemolysis assay (results presented in chapter 4). It is possible that the variola virus homologue possesses greater activity (possibly binding affinity) against human/baboon complement than rVCP. If the data obtained from this study matches that of the preliminary data, it will not only bring up interesting questions about host specificity but also lend valuable information for structure-function determination of the complement binding residues. If a difference in the proteins' binding kinetics to human or rat $\mathrm{C} 3 \mathrm{~b}$ is found, then the only twelve amino acid dissimilarity between rVCP and the variola virus homologue of VCP will help narrow down the important residues involved in this interaction. 


\section{rVCP Cell Binding and Kinetic Studies Using Electron Microscopy}

In order to investigate the cell binding and kinetics of rVCP, electron microscopy (EM) will be used. Brendon Price, a collaborator from the Electron Microscope Unit, University of Cape Town, Rondebosch, South Africa, will carry out the EM experiments. Recombinant VCP, expressed from the Pichia pastoris yeast expression system, will be purified by heparin affinity chromatography as described in chapter 2. Using HUVECs grown to confluency in 6-well plates, various concentrations of rVCP will be incubated with the cells for various time points. Following incubation, the cells will be fixed, embedded, processed and viewed by EM. The cells will be treated with chicken antiVCP IgY primary antibody, followed by biotinylated goat anti-chicken IgG secondary antibody and finally visualized using conjugated streptavidin- $5 \mathrm{~nm}$ gold. The position of the gold particles will be tracked to determine the location of rVCP, initially on the cell surface, and possibly within the cell at a later time point. The data obtained from this study will greatly increase our understanding of the kinetics and intracellular localization of rVCP following its binding to cell surface heparan sulfate. This data may be useful for increasing the serum half-life of rVCP in vivo as well as providing a basic understanding

of how the protein behaves after it is secreted from the vaccinia virus-infected cell during the natural infection process. 


\section{$\underline{\text { Pharmacokinetic and Xenotransplantation Studies in Baboons }}$}

To determine the pharmacokinetics of rVCP in baboons, several baboons will receive large bolus I.V. injections and the serum $\mathrm{CH} 50$ will be monitored over time. The baboons and technical assistance will be provided by Professor Del Kahn, head of the Surgical Transplant Service, University of Cape Town, Rondebosch, South Africa. As described in chapter $2, \mathrm{rVCP}$ will be expressed and purified by heparin affinity chromatography. The kinetics of rVCP in the serum, determined by $\mathrm{CH} 50$, will be compared to that observed in the rat studies (see chapter 6). The dosage of rVCP required to maintain a serum $\mathrm{CH} 50$ below 10 for an extended time (possibly several weeks) will be determined using the data collected. The dosage of the variola virus homologue of VCP required to maintain a serum $\mathrm{CH} 50$ below 10 will also be evaluated, once it is cloned and produced. An internal I.V. pump will be purchased and used to continuously deliver the required quantity of VCP for the desired time. Once a satisfactory $\mathrm{rVCP}$ regimen and delivery strategy is achieved, pig to baboon xenotransplants can be attempted. Pig livers will be transplanted into baboons, with and without rVCP. Organ survival, histology and immunohistochemistry (for VCP, C3, and $\operatorname{IgM}$ ) will be used to evaluate the efficacy of rVCP in blocking hyperacute rejection. 


\section{C3b/rVCP and Heparin/rVCP Co-crystallization Studies}

Dr. Jamie Rossjohn, St. Vincent's Institute of Medical Research, Fitzroy, Australia will perform the co-crystallization of rVCP and C3b. C3 will be purified from human serum and partially trypsinized to form $\mathrm{C} 3 \mathrm{~b}$, as described in chapter 4 .

Recombinant VCP will be produced using the Pichia pastoris yeast expression system and purified to nearly $100 \%$ by repeated heparin affinity chromatography (described in chapter 2). The resulting crystal structure will provide conclusive results about the residues involved in $\mathrm{rVCP}$ binding to $\mathrm{C} 3 \mathrm{~b}$. These results will determine whether the complement binding residues defined by the hemolysis assay, SPR binding analysis, and homology with MCP, do in fact play a role in this interaction.

Dr. Krishna Murthy from the Center for Biophysical Science and Engineering, University of Alabama at Birmingham, who performed the X-ray crystallography of rVCP, will conduct the co-crystallization of rVCP and heparin. Again, rVCP will be expressed using the Pichia pastoris yeast expression system and purified to nearly $100 \%$ repeated heparin affinity chromatography (described in chapter 2). Using low molecular weight heparin, approximately $5 \mathrm{kDa}$ molecular size (Sigma), rVCP will be cocrystallized with heparin. The resulting structure will provide conclusive results about the residues involved in $\mathrm{rVCP}$ binding to heparin. This will determine whether the heparin binding sites defined by chromatography, SPR binding analysis, and homology with FGF, do in fact play a role in this interaction. 


\section{C3b/rVCP and Heparin/rVCP Binding Kinetics Using Biacore}

Biacore kinetics analyses between $\mathrm{C} 3 \mathrm{~b}$ and $\mathrm{rVCP}$, as well as Heparin and rVCP will be performed in collaboration with Dr. Shrihari Arjunwadkar, from the Department of Microbiology, Division of Virology, University of Cape Town, South Africa. With C3b immobilized to the surface of a CM5 sensor chip, various concentrations $(0.5$ - 50 $\mathrm{nM}$ ) of rVCP will be passed over the surface and binding will be measured. The binding plots will be overlaid and fit using the BIAevaluation software package. The software will determine the $K_{a}$ and $K_{d}$ of this interaction automatically. Similarly, with low molecular weight heparin immobilized to the surface of a sensor chip, various concentrations $(0.5-50 \mathrm{nM})$ of rVCP will be injected and binding will be measured. Again, the binding plots will be overlaid and fit using the BIAevaluation software system. Again, the software will determine the $\mathrm{K}_{\mathrm{a}}$ and $\mathrm{K}_{\mathrm{d}}$ of this interaction automatically. The association and disassociation constants for rVCP binding to complement and heparin may provide very useful insights into these interactions. By studying the kinetics of mammalian complement control proteins, knowledge about the evolutionary history and the potency of VCP can be learned. 


\section{Identification of C3 Fragments Involved in rVCP Binding Using Biacore}

In collaboration with Dr. Seppo Meri from the Haartman Institute, University of Helsinki, Finland, surface plasmon resonance analysis will be used to identify the regions of $\mathrm{C} 3$ involved in binding rVCP. Human $\mathrm{C} 3$ will be purified from human serum as described in chapter 4 . The $\mathrm{C} 3 \mathrm{~b}$ fragment of $\mathrm{C} 3$ will be produced from partial trypsinization, as described in chapter 4 . The $\mathrm{C} 3 \mathrm{c}$ fragment will be purified using $\mathrm{C} 3$ and Activated Thiol Sepharose 4B (Pharmacia) as described previously (Jokiranta et al., 2001). Lastly, the $\mathrm{C} 3 \mathrm{~d}$ fragment of $\mathrm{C} 3$ will be generated and purified from $\mathrm{C} 3 \mathrm{using}$ a procedure described previously (Jokiranta et al., 2000). SPR binding studies will be performed using a Biacore 2000 instrument and the data will be analyzed using the BIAevaluation software package. C3 and each of the C3 fragments, C3b, C3c, and C3d will be immobilized to the surface of a CM5 sensor chip using standard amine coupling procedures described in chapter 4. Various concentrations of rVCP, expressed and purified by heparin affinity chromatography, will be injected over the surface of each sensor chip to determine whether binding occurs. The ability of rVCP to bind, as well as the relative strength of the binding, to $\mathrm{C} 3$ or the fragments of $\mathrm{C} 3$ will help identify the region(s) within $\mathrm{C} 3$ required for this interaction to occur. 


\section{Protein Engineering and Use of VCP as an Immunomodulatory Agent}

Complement has been shown to play an important role in many inflammatory conditions, such as Alzheimer's disease, rheumatoid arthritis, CNS injury and xenotransplant rejection. In hopes of developing a therapeutic agent for use in treating such conditions, various complement-inhibiting proteins have been studied extensively for many years. There have been proteins identified that are capable of blocking the complement cascade at nearly every step, from $\mathrm{C} 1$ (C1-Inhibitor) to the assembly of the membrane attack complex (sCD59), and a few proteins that can block at multiple steps (sCR1). Many of these are membrane bound mammalian proteins used to prevent unwanted complement activation and tissue destruction in the absence of an invading microorganism. In order to use them as a therapeutic agent, however, their transmembrane domain is removed and the protein expressed in a soluble form. These soluble proteins are often very affective in blocking complement, both in vitro as well as in vivo. However, the soluble mammalian complement control proteins are very large, generally consisting of 20 SCRs or more (>150 kDa molecular size). VCP is the smallest known complement regulatory protein, possessing a similar function as exhibited by sCR1. In addition, VCP expands its repertoire of functions by including heparin binding sites, making it both small and multifunctional.

It has been shown previously that VCP exhibits greater potency in inhibiting complement than an equal concentration of C4b-BP (Kotwal, 1994). This shows that the small structure of VCP may improve its ability to bind the complement components $\mathrm{C} 3 \mathrm{~b}$ and $\mathrm{C} 4 \mathrm{~b}$, thus improving its ability to block complement. By determining the residues involved in this interaction with complement, a better understanding of the structural 
basis for this potency may be acquired. With the residues involved in both binding complement and facilitating its cleavage to an inactivated form by factor I determined, the structure of a complement inhibitor even smaller and possibly more potent than VCP may be created by protein engineering. A short protein backbone can be created to display these critical residues in the specific spatial orientation displayed in VCP, required for complement binding. With the size of the complement inhibitor reduced, absorption into the blood may become possible, allowing for I.P., SubQ, or I.M. administration to be effective. In addition, if the synthetic protein can be made small enough, it may be able to pass across the blood brain barrier into the cerebral spinal fluid. With this being possible, damaging inflammation mediated by complement activation shortly following CNS injury may be modulated, perhaps resulting in significant neuronal sparing and return of neurological function.

The heparin binding ability of VCP endows it with many extremely novel functions, including the ability to block chemokine induced monocyte migration and preventing molecular and cellular interactions with cells. Knowing the amino acids and their spatial orientations involved in VCP's ability to bind heparin may someday allow for the development of a short synthetic peptide, able to retain the novel functions displayed by VCP. This synthetic peptide may be ten amino acids or less, and still maintain the ability to bind heparin and heparan sulfate proteoglycans, allowing for steric interference with unwanted molecular and cellular interactions with cells. A synthetic peptide with this immunomodulatory ability could have therapeutic uses for preventing molecular and cellular mechanisms of graft rejection or even autoimmune diseases. It could prove valuable in prolonging survival of xenografted organs by inhibiting cellular mechanisms 
of graft rejection associated with accelerated rejection and acute cellular rejection processes. The immune modulating ability that would be displayed by this peptide may be able to suppress an autoimmune reaction in diseases such as rheumatoid arthritis, systemic lupus erythematosus, and multiple sclerosis. However, there is no indication of how long the peptide would be effective or if any side effects would result from extended use.

By using the complement and heparin binding regions identified within VCP, small synthetic therapeutic proteins, created by protein engineering, may be developed for use against many complement-mediated conditions. This strategy would be less complex when designing a synthetic heparin binding peptide, and much more complicated when creating a synthetic complement binding protein. Due to the fact that residues involved in binding complement are spread throughout nearly the entire length of VCP, it seems very likely that the vaccinia virus has already reduce the protein to its smallest functional unit. It would therefore be exceedingly difficult to further reduce the number of amino acids making up the protein. Spatially, four SCRs may be the minimal requirement for binding $\mathrm{C} 3 \mathrm{~b}$ and $\mathrm{C} 4 \mathrm{~b}$, and facilitating their cleavage by factor I. If this were in fact the case, VCP would remain a likely candidate for therapeutic use in blocking the harmful effects of complement seen in many inflammatory diseases. 


\section{REFERENCES}

Al-Mohanna, Parhar, R., \& Kotwal, G. J. (2001) Vaccinia virus complement control protein is capable of protecting Xenoendothelial cells from antibody binding and killing by human complement and cytotoxic cells. Transplantation 71: 796-801.

Alcami, A. \& Smith, G. L. (1992) A soluble receptor for interleukin-1 beta encoded by vaccinia virus: a novel mechanism of virus modulation of the host response to infection. Cell 71: 153-167.

Barlow, P. N., Baron, M., Norman, D. G., Day, A. J., Willis, A. C., Sim, R. B., \& Campbell, I. D. (1991) Secondary structure of a complement control protein module by two-dimensional 1H NMR. Biochemistry 30: 997-1004.

Barlow, P. N., Norman, D. G., Steinkasserer, A., Horne, T. J., Pearce, J., Driscoll, P. C., Sim, R. B., \& Campbell, I. D. (1992) Solution structure of the fifth repeat of factor H: a second example of the complement control protein module. Biochemistry 31: 36263634.

Barlow, P. N., Steinkasserer, A., Norman, D. G., Kieffer, B., Wiles, A. P., Sim, R. B., \& Campbell, I. D. (1993) Solution structure of a pair of complement modules by nuclear magnetic resonance. J. Mol. Biol. 232: 268-284.

Beaud, G. (1995) Vaccinia virus DNA replication: a short review. Biochimie 77: 774779.

Berger, J. M. (1998) Structure of DNA topoisomerases. Biochim.Biophys.Acta 1400: 318.

Blackmore, T. K., Hellwage, J., Sadlon, T. A., Higgs, N., Zipfel, P. F., Ward, H. M., \& Gordon, D. L. (1998) Identification of the second heparin-binding domain in human complement factor H. J. Immunol 160: 3342-3348.

Blom, A. M., Webb, J., Villoutreix, B. O., \& Dahlback, B. (1999) A cluster of positively charged amino acids in the C4BP alpha-chain is crucial for $\mathrm{C} 4 \mathrm{~b}$ binding and factor $\mathrm{I}$ cofactor function. J. Biol. Chem. 274: 19237-19245.

Bouma, B., de Groot, P. G., van den Elsen, J. M., Ravelli, R. B., Schouten, A., Simmelink, M. J., Derksen, R. H., Kroon, J., \& Gros, P. (1999) Adhesion mechanism of human beta(2)-glycoprotein I to phospholipids based on its crystal structure.

EMBO J. 18: 5166-5174. 
Brenner, B. M., Hostetter, T. H., \& Humes, H. D. (1978) Glomerular permselectivity: barrier function based on discrimination of molecular size and charge. Am. J. Physiol 234: F455-F460.

Cardin, A. D., Demeter, D. A., Weintraub, H. J., \& Jackson, R. L. (1991) Molecular design and modeling of protein-heparin interactions. Methods Enzymol. 203:556-83: 556-583.

Cardin, A. D. \& Weintraub, H. J. (1989) Molecular modeling of proteinglycosaminoglycan interactions. Arteriosclerosis 9: 21-32.

Carson, E. R. \& Jones, E. A. (1979) Use of kinetic analysis and mathematical modeling in the study of metabolic pathways in vivo. Applications to hepatic organic anion metabolism. (First of two parts). N. Engl. J. Med. 300: 1016-1027.

Casasnovas, J. M., Larvie, M., \& Stehle, T. (1999) Crystal structure of two CD46 domains reveals an extended measles virus-binding surface. EMBO J. 18: 2911-2922.

Chung, C. S., Hsiao, J. C., Chang, Y. S., \& Chang, W. (1998) A27L protein mediates vaccinia virus interaction with cell surface heparan sulfate. J. Virol. 72: 1577-1585.

Daly, J. \& Kotwal, G. J. (1998) Pro-inflammatory complement activation by the A beta peptide of Alzheimer's disease is biologically significant and can be blocked by vaccinia virus complement control protein. Neurobiol. Aging 19: 619-627.

Deane, D., McInnes, C. J., Percival, A., Wood, A., Thomson, J., Lear, A., Gilray, J., Fleming, S., Mercer, A., \& Haig, D. (2000) Orf virus encodes a novel secreted protein inhibitor of granulocyte- macrophage colony-stimulating factor and interleukin-2. $J$. Virol. 74: 1313-1320.

DiGabriele, A. D., Lax, I., Chen, D. I., Svahn, C. M., Jaye, M., Schlessinger, J., \& Hendrickson, W. A. (1998) Structure of a heparin-linked biologically active dimer of fibroblast growth factor. Nature 393: 812-817.

DuBose, D. A. \& Haugland, R. (1993) Comparisons of endothelial cell G- and F-actin distribution in situ and in vitro. Biotech. Histochem. 68: 8-16.

Faham, S., Hileman, R. E., Fromm, J. R., Linhardt, R. J., \& Rees, D. C. (1996) Heparin structure and interactions with basic fibroblast growth factor. Science 271: 11161120.

Fleming, S. B., Haig, D. M., Nettleton, P., Reid, H. W., McCaughan, C. A., Wise, L. M., $\&$ Mercer, A. (2000) Sequence and functional analysis of a homolog of interleukin-10 encoded by the parapoxvirus orf virus [In Process Citation]. Virus Genes 21: 85-95. 
Flodgaard, H., Ostergaard, E., Bayne, S., Svendsen, A., Thomsen, J., Engels, M., \& Wollmer, A. (1991) Covalent structure of two novel neutrophile leucocyte-derived proteins of porcine and human origin. Neutrophile elastase homologues with strong monocyte and fibroblast chemotactic activities. Eur. J. Biochem. 197: 535-547.

Gaboriaud, C., Rossi, V., Bally, I., Arlaud, G. J., \& Fontecilla-Camps, J. C. (2000) Crystal structure of the catalytic domain of human complement c1s: a serine protease with a handle. EMBO J. 19: 1755-1765.

Gill, S. C. \& von Hippel, P. H. (1989) Calculation of protein extinction coefficients from amino acid sequence data. Anal. Biochem. 182: 319-326.

Goodfellow, R. M., Williams, A. S., Levin, J. L., Williams, B. D., \& Morgan, B. P. (2000) Soluble complement receptor one (sCR1) inhibits the development and progression of rat collagen-induced arthritis. Clin. Exp. Immunol 119: 210-216.

Graham, K. A., Lalani, A. S., Macen, J. L., Ness, T. L., Barry, M., Liu, L. Y., Lucas, A., Clark-Lewis, I., Moyer, R. W., \& McFadden, G. (1997) The T1/35kDa family of poxvirus-secreted proteins bind chemokines and modulate leukocyte influx into virusinfected tissues. Virology 229: 12-24.

Gresham, H. D., Matthews, D. F., \& Griffin, F. M., Jr. (1986) Isolation of human complement component C3 from small volumes of plasma. Anal. Biochem. 154: 454459.

Henderson, C. E., Bromek, K., Mullin, N. P., Smith, B. O., Uhrin, D., \& Barlow, P. N. (2001) Solution structure and dynamics of the central CCP module pair of a poxvirus complement control protein. J. Mol. Biol. 307: 323-339.

Hershfield, M. S., Buckley, R. H., Greenberg, M. L., Melton, A. L., Schiff, R., Hatem, C., Kurtzberg, J., Markert, M. L., Kobayashi, R. H., Kobayashi, A. L., \& . (1987) Treatment of adenosine deaminase deficiency with polyethylene glycol-modified adenosine deaminase. N. Engl. J. Med. 316: 589-596.

Hessing, M., Vlooswijk, R. A., Hackeng, T. M., Kanters, D., \& Bouma, B. N. (1990) The localization of heparin-binding fragments on human $\mathrm{C} 4 \mathrm{~b}$-binding protein. J. Immunol 144: 204-208.

Hollinshead, M., Vanderplasschen, A., Smith, G. L., \& Vaux, D. J. (1999) Vaccinia virus intracellular mature virions contain only one lipid membrane. J. Virol. 73: 1503-1517.

Howard, J., Justus, D. E., Totmenin, A. V., Shchelkunov, S., \& Kotwal, G. J. (1998) Molecular mimicry of the inflammation modulatory proteins (IMPs) of poxviruses: evasion of the inflammatory response to preserve viral habitat. J. Leukoc. Biol. 64: $68-71$. 
Hwang, T. L. \& Shaka, A. J. (1998) Multiple-pulse mixing sequences that selectively enhance chemical exchange or cross-relaxation peaks in high-resolution NMR spectra. J. Magn. Reson. 135: 280-287.

Johnson, G. P., Goebel, S. J., \& Paoletti, E. (1993) An update on the vaccinia virus genome. Virology 196: 381-401.

Jokiranta, T. S., Hellwage, J., Koistinen V., Zipfel P. F., \& Meri, S. (2000) Each of the three binding sites on factor $\mathrm{H}$ interacts with a distinct site on C3b. J. Biol. Chem. 275:27657-27662.

Jokiranta, T. S., Westin, J., Nilsson, U. R., Nilsson, B., Hellwage, J., Lofas, S., Gordon, D. L., Ekdahl, K. N., \& Meri, S. (2001) Complement C3b interactions studied with surface plasmon resonance technique. Int. Immunopharmacol. 1: 495-506.

Kettle, S., Alcami, A., Khanna, A., Ehret, R., Jassoy, C., \& Smith, G. L. (1997) Vaccinia virus serpin B13R (SPI-2) inhibits interleukin-1beta- converting enzyme and protects virus-infected cells from TNF- and Fas- mediated apoptosis, but does not prevent IL1beta-induced fever. J. Gen. Virol. 78 (Pt 3): 677-685.

Kirkitadze, M. D., Henderson, C., Price, N. C., Kelly, S. M., Mullin, N. P., Parkinson, J., Dryden, D. T., \& Barlow, P. N. (1999) Central modules of the vaccinia virus complement control protein are not in extensive contact. Biochem. J. 344 Pt 1: 167175.

Kotwal, G. J. \& Moss, B. (1988) Vaccinia virus encodes a secretory polypeptide structurally related to complement control proteins. Nature 335: 176-178.

Kotwal, G. J. \& Moss, B. (1989) Vaccinia virus encodes two proteins that are structurally related to members of the plasma serine protease inhibitor superfamily [published erratum appears in J Virol 1990 Feb;64(2):966]. J. Virol. 63: 600-606.

Kotwal, G. J., Isaacs, S. N., McKenzie, R., Frank, M. M., \& Moss, B. (1990) Inhibition of the complement cascade by the major secretory protein of vaccinia virus. Science 250: $827-830$.

Kotwal, G. J. (1994) Purification of virokines using ultrafiltration. Am. Biotechnol. Lab. 12: 76-77.

Kotwal, G. J. (1997) Microorganisms and their interaction with the immune system. $J$. Leukoc. Biol. 62: 415-429.

Kotwal, G. J., Miller, C. G., \& Justus, D. E. (1998a) The inflammation modulatory protein (IMP) of cowpox virus drastically diminishes the tissue damage by downregulating cellular infiltration resulting from complement activation. Mol. Cell Biochem. 185: 39-46. 
Kotwal, G. J., Reynolds, D. N., Keeling, K. L., Howard, J., \& Justus, D. E. (1998b) Vaccinia virus complement control proteinis a virokine with lysozyme-like heparin binding activity: possible implications in prolonged evasion of host immune response. In: Proc.10th Int.Congress of Immunol. (Monduzzi Editore Publishing, ed.), pp. 315320.

Kotwal, G. J. (2000) Poxviral mimicry of complement and chemokine system components: what's the end game? Immunol. Today 21: 242-248.

Lalani, A. S., Graham, K., Mossman, K., Rajarathnam, K., Clark-Lewis, I., Kelvin, D., \& McFadden, G. (1997) The purified myxoma virus gamma interferon receptor homolog M-T7 interacts with the heparin-binding domains of chemokines. J. Virol. 71: 4356-4363.

Lanteri, M. B., Powell, M. S., Christiansen, D., Li, Y. Q., Hogarth, M., Sandrin, M. S., Mckenzie, I. F., \& Loveland, B. E. (2000) Inhibition of hyperacute transplant rejection by soluble proteins with the functional domains of CD46 and FcgammaRII. Transplantation 69: 1128-1136.

Lian, R. H., Kotwal, G. J., Wellhausen, S. R., Hunt, L. A., \& Justus, D. E. (1996) IFNgamma-induced MHC class II gene expression is suppressed in endothelial cells by dextran sulfate. J. Immunol. 157: 864-873.

Liszewski, M. K., Leung, M., Cui, W., Subramanian, V. B., Parkinson, J., Barlow, P. N., Manchester, M., \& Atkinson, J. P. (2000) Dissecting sites important for complement regulatory activity in membrane cofactor protein (MCP; CD46). J. Biol. Chem. 275: 37692-37701.

Lomas, D. A., Evans, D. L., Upton, C., McFadden, G., \& Carrell, R. W. (1993) Inhibition of plasmin, urokinase, tissue plasminogen activator, and $\mathrm{C} 1 \mathrm{~S}$ by a myxoma virus serine proteinase inhibitor. J. Biol. Chem. 268: 516-521.

Loparev, V. N., Parsons, J. M., Knight, J. C., Panus, J. F., Ray, C. A., Buller, R. M., Pickup, D. J., \& Esposito, J. J. (1998) A third distinct tumor necrosis factor receptor of orthopoxviruses. Proc. Natl. Acad. Sci. U.S.A. 95: 3786-3791.

Margalit, H., Fischer, N., \& Ben Sasson, S. A. (1993) Comparative analysis of structurally defined heparin binding sequences reveals a distinct spatial distribution of basic residues. J. Biol. Chem. 268: 19228-19231.

Marino, M., Friedlander, J. A., McCluskey, R. T., \& Andrews, D. (1999) Identification of a heparin-binding region of rat thyroglobulin involved in megalin binding [In Process Citation]. J. Biol. Chem. 274: 30377-30386.

McKenzie, R., Kotwal, G. J., Moss, B., Hammer, C. H., \& Frank, M. M. (1992) Regulation of complement activity by vaccinia virus complement-control protein. $J$. Infect. Dis. 166: 1245-1250. 
Mehvar, R. (2000) Modulation of the pharmacokinetics and pharmacodynamics of proteins by polyethylene glycol conjugation. J. Pharm. Pharm.Sci. 3: 125-136.

Meri, S. \& Pangburn, M. K. (1994) Regulation of alternative pathway complement activation by glycosaminoglycans: specificity of the polyanion binding site on factor H. Biochem.Biophys. Res. Commun. 198: 52-59.

Miller, C. G., Justus, D. E., Jayaraman, S., \& Kotwal, G. J. (1995) Severe and prolonged inflammatory response to localized cowpox virus infection in footpads of C5deficient mice: investigation of the role of host complement in poxvirus pathogenesis. Cell Immunol. 162: 326-332.

Miller, C. G., Shchelkunov, S. N., \& Kotwal, G. J. (1997) The cowpox virus-encoded homolog of the vaccinia virus complement control protein is an inflammation modulatory protein. Virology 229: 126-133.

Mizuno, M., Nishikawa, K., Morgan, B. P., \& Matsuo, S. (2000) Comparison of the suppressive effects of soluble CR1 and C5a receptor antagonist in acute arthritis induced in rats by blocking of CD59. Clin. Exp. Immunol 119: 368-375.

Molina, H., Perkins, S. J., Guthridge, J., Gorka, J., Kinoshita, T., \& Holers, V. M. (1995) Characterization of a complement receptor 2 (CR2, CD21) ligand binding site for C3. An initial model of ligand interaction with two linked short consensus repeat modules. J. Immunol 154: 5426-5435.

Moss, B. (1996) Poxviridae: The Viruses and Their Replication. In: Fundamental Virology (Bernard N.Fields, David M.Knipe, \& Peter M.Howley, eds.), pp. 11631197. Lippincott-Raven.

Nagar, B., Jones, R. G., Diefenbach, R. J., Isenman, D. E., \& Rini, J. M. (1998) X-ray crystal structure of C3d: a C3 fragment and ligand for complement receptor 2. Science 280: 1277-1281.

Nash, P., Barrett, J., Cao, J. X., Hota-Mitchell, S., Lalani, A. S., Everett, H., Xu, X. M., Robichaud, J., Hnatiuk, S., Ainslie, C., Seet, B. T., \& McFadden, G. (1999) Immunomodulation by viruses: the myxoma virus story. Immunol. Rev. 168:103-20: 103-120.

Pangburn, M. K., Pangburn, K. L., Koistinen, V., Meri, S., \& Sharma, A. K. (2000) Molecular mechanisms of target recognition in an innate immune system: interactions among factor $\mathrm{H}, \mathrm{C} 3 \mathrm{~b}$, and target in the alternative pathway of human complement. $J$. Immunol 164: 4742-4751.

Piddlesden, S. J., Storch, M. K., Hibbs, M., Freeman, A. M., Lassmann, H., \& Morgan, B. P. (1994) Soluble recombinant complement receptor 1 inhibits inflammation and demyelination in antibody-mediated demyelinating experimental allergic encephalomyelitis. J. Immunol 152: 5477-5484. 
Pratt, J. R., Harmer, A. W., Levin, J., \& Sacks, S. H. (1997) Influence of complement on the allospecific antibody response to a primary vascularized organ graft. Eur. J. Immunol 27: 2848-2853.

Pratt, J. R., Hibbs, M. J., Laver, A. J., Smith, R. A., \& Sacks, S. H. (1996) Effects of complement inhibition with soluble complement receptor-1 on vascular injury and inflammation during renal allograft rejection in the rat. Am. J. Pathol. 149: 20552066.

Press, E. M. \& Gagnon, J. (1981) Human complement component C4. Structural studies on the fragments derived from $\mathrm{C} 4 \mathrm{~b}$ by cleavage with $\mathrm{C} 3 \mathrm{~b}$ inactivator. Biochem. J. 199: 351-357.

Privalov, P. L. \& Potekhin, S. A. (1986) Scanning microcalorimetry in studying temperature-induced changes in proteins. Methods Enzymol. 131: 4-51.

Prodinger, W. M., Schwendinger, M. G., Schoch, J., Kochle, M., Larcher, C., \& Dierich, M. P. (1998) Characterization of C3dg binding to a recess formed between short consensus repeats 1 and 2 of complement receptor type 2 (CR2; CD21). J. Immunol 161: 4604-4610.

Quan, L. T., Caputo, A., Bleackley, R. C., Pickup, D. J., \& Salvesen, G. S. (1995) Granzyme B is inhibited by the cowpox virus serpin cytokine response modifier A. $J$. Biol. Chem. 270: 10377-10379.

Reboul, A., Thielens, N., Villiers, M. B., \& Colomb, M. G. (1979) Purification of human complement subcomponent C4. C4 cleavage by C1s. FEBS Lett. 103: 156-161.

Reddy, K. R. (2000) Controlled-release, pegylation, liposomal formulations: new mechanisms in the delivery of injectable drugs. Ann. Pharmacother. 34: 915-923.

Reddy, K. R., Wright, T. L., Pockros, P. J., Shiffman, M., Everson, G., Reindollar, R., Fried, M. W., Purdum, P. P., III, Jensen, D., Smith, C., Lee, W. M., Boyer, T. D., Lin, A., Pedder, S., \& DePamphilis, J. (2001) Efficacy and safety of pegylated (40-kd) interferon alpha-2a compared with interferon alpha-2a in noncirrhotic patients with chronic hepatitis C. Hepatology 33: 433-438.

Regal, J. F., Fraser, D. G., \& Toth, C. A. (1993) Role of the complement system in antigen-induced bronchoconstriction and changes in blood pressure in the guinea pig. J. Pharmacol. Exp. Ther. 267: 979-988.

Reynolds, D. N., Keeling, K. L., Molestina, R., Srisatjaluk, R., Butterfield, J. H., Ehringer, W., Justus, D. E., \& Kotwal, G. J. (2000) Heparin binding activity of vaccinia virus complement control protein confers additional properties of uptake by mast cells and attatchment to endothelial cells. In: Advances in Animal Virology, Oxford and IBH, New York (S. Jameel \& Lambris, J. D., eds.), pp. 337-342. 
Rosengard, A. M., Alonso, L. C., Korb, L. C., Baldwin, W. M., III, Sanfilippo, F., Turka, L. A., \& Ahearn, J. M. (1999) Functional characterization of soluble and membranebound forms of vaccinia virus complement control protein (VCP). Mol. Immunol 36: 685-697.

Sahu, A., Isaacs, S. N., Soulika, A. M., \& Lambris, J. D. (1998) Interaction of vaccinia virus complement control protein with human complement proteins: factor I-mediated degradation of $\mathrm{C} 3 \mathrm{~b}$ to $\mathrm{iC} 3 \mathrm{~b} 1$ inactivates the alternative complement pathway. $J$. Immunol. 160: 5596-5604.

Sali, A. \& Blundell, T. L. (1993) Comparative protein modelling by satisfaction of spatial restraints. J. Mol. Biol. 234: 779-815.

Schlessinger, J., Plotnikov, A. N., Ibrahimi, O. A., Eliseenkova, A. V., Yeh, B. K., Yayon, A., Linhardt, R. J., \& Mohammadi, M. (2000) Crystal structure of a ternary FGF-FGFR-heparin complex reveals a dual role for heparin in FGFR binding and dimerization. Mol. Cell 6: 743-750.

Schwarzenbacher, R., Zeth, K., Diederichs, K., Gries, A., Kostner, G. M., Laggner, P., \& Prassl, R. (1999) Crystal structure of human beta2-glycoprotein I: implications for phospholipid binding and the antiphospholipid syndrome. EMBO J. 18: 6228-6239.

Sendak, R. A. \& Bensadoun, A. (1998) Identification of a heparin-binding domain in the distal carboxyl- terminal region of lipoprotein lipase by site-directed mutagenesis. $J$. Lipid Res. 39: 1310-1315.

Senkevich, T. G., Bugert, J. J., Sisler, J. R., Koonin, E. V., Darai, G., \& Moss, B. (1996) Genome sequence of a human tumorigenic poxvirus: prediction of specific host response-evasion genes. Science 273: 813-816.

Sharma, A. K. \& Pangburn, M. K. (1996) Identification of three physically and functionally distinct binding sites for $\mathrm{C} 3 \mathrm{~b}$ in human complement factor $\mathrm{H}$ by deletion mutagenesis. Proc. Natl. Acad. Sci.U.S.A 93: 10996-11001.

Shchelkunov, S. N., Safronov, P. F., Totmenin, A. V., Petrov, N. A., Ryazankina, O. I., Gutorov, V. V., \& Kotwal, G. J. (1998) The genomic sequence analysis of the left and right species-specific terminal region of a cowpox virus strain reveals unique sequences and a cluster of intact ORFs for immunomodulatory and host range proteins. Virology 243: 432-460.

Smith, V. P., Bryant, N. A., \& Alcami, A. (2000) Ectromelia, vaccinia and cowpox viruses encode secreted interleukin-18- binding proteins. J. Gen. Virol. Pt 5:1223-30.

Spriggs, M. K., Hruby, D. E., Maliszewski, C. R., Pickup, D. J., Sims, J. E., Buller, R. M., \& VanSlyke, J. (1992) Vaccinia and cowpox viruses encode a novel secreted interleukin-1- binding protein. Cell 71: 145-152. 
Tack, B. F., Harrison, R. A., Janatova, J., Thomas, M. L., \& Prahl, J. W. (1980) Evidence for presence of an internal thiolester bond in third component of human complement. Proc. Natl. Acad. Sci. U.S.A 77: 5764-5768.

Tewari, M., Beidler, D. R., \& Dixit, V. M. (1995) CrmA-inhibitable cleavage of the 70$\mathrm{kDa}$ protein component of the U1 small nuclear ribonucleoprotein during Fas- and tumor necrosis factor- induced apoptosis. J. Biol. Chem. 270: 18738-18741.

Turner, P. C., Sancho, M. C., Thoennes, S. R., Caputo, A., Bleackley, R. C., \& Moyer, R. W. (1999) Myxoma virus Serp2 is a weak inhibitor of granzyme B and interleukin1beta-converting enzyme in vitro and unlike CrmA cannot block apoptosis in cowpox virus-infected cells. J. Virol. 73: 6394-6404.

Upton, C., Macen, J. L., Schreiber, M., \& McFadden, G. (1991) Myxoma virus expresses a secreted protein with homology to the tumor necrosis factor receptor gene family that contributes to viral virulence. Virology 184: 370-382.

Upton, C., Macen, J. L., Wishart, D. S., \& McFadden, G. (1990) Myxoma virus and malignant rabbit fibroma virus encode a serpin-like protein important for virus virulence. Virology 179: 618-631.

Upton, C., Mossman, K., \& McFadden, G. (1992) Encoding of a homolog of the IFNgamma receptor by myxoma virus. Science 258: 1369-1372.

Vacheron, F., Rudent, A., Perin, S., Labarre, C., Quero, A. M., \& Guenounou, M. (1990) Production of interleukin 1 and tumour necrosis factor activities in bronchoalveolar washings following infection of mice by influenza virus. J. Gen. Virol. 71: 477-479.

Wang, Y. S., Youngster, S., Bausch, J., Zhang, R., McNemar, C., \& Wyss, D. F. (2000) Identification of the major positional isomer of pegylated interferon alpha- $2 \mathrm{~b}$.

Biochemistry 39: 10634-10640.

Wiles, A. P., Shaw, G., Bright, J., Perczel, A., Campbell, I. D., \& Barlow, P. N. (1997) NMR studies of a viral protein that mimics the regulators of complement activation. J. Mol. Biol. 272: 253-265.

Wilton, S., Mohandas, A. R., \& Dales, S. (1995) Organization of the vaccinia envelope and relationship to the structure of intracellular mature virions. Virology 214: 503511.

Wong, P., Hampton, B., Szylobryt, E., Gallagher, A. M., Jaye, M., \& Burgess, W. H. (1995) Analysis of putative heparin-binding domains of fibroblast growth factor-1. Using site-directed mutagenesis and peptide analogues. J. Biol. Chem. 270: 2580525811.

Xiang, Y. \& Moss, B. (1999) IL-18 binding and inhibition of interferon gamma induction by human poxvirus-encoded proteins. Proc. Natl. Acad. Sci. U.S.A. 96: 11537-11542. 


\section{APPENDIX}


Figure 63. Rat serum CH50 following 24 mg/kg SubQ bolus. Following a subcutaneous bolus dose of $24 \mathrm{mg} / \mathrm{kg}$, the complement activity was monitored periodically by CH50 assay. The CH50 was measured every half-hour for four hours. As can be seen, no evident change in complement activity is evident. The verticle black line represents $\mathrm{CH} 50$. 


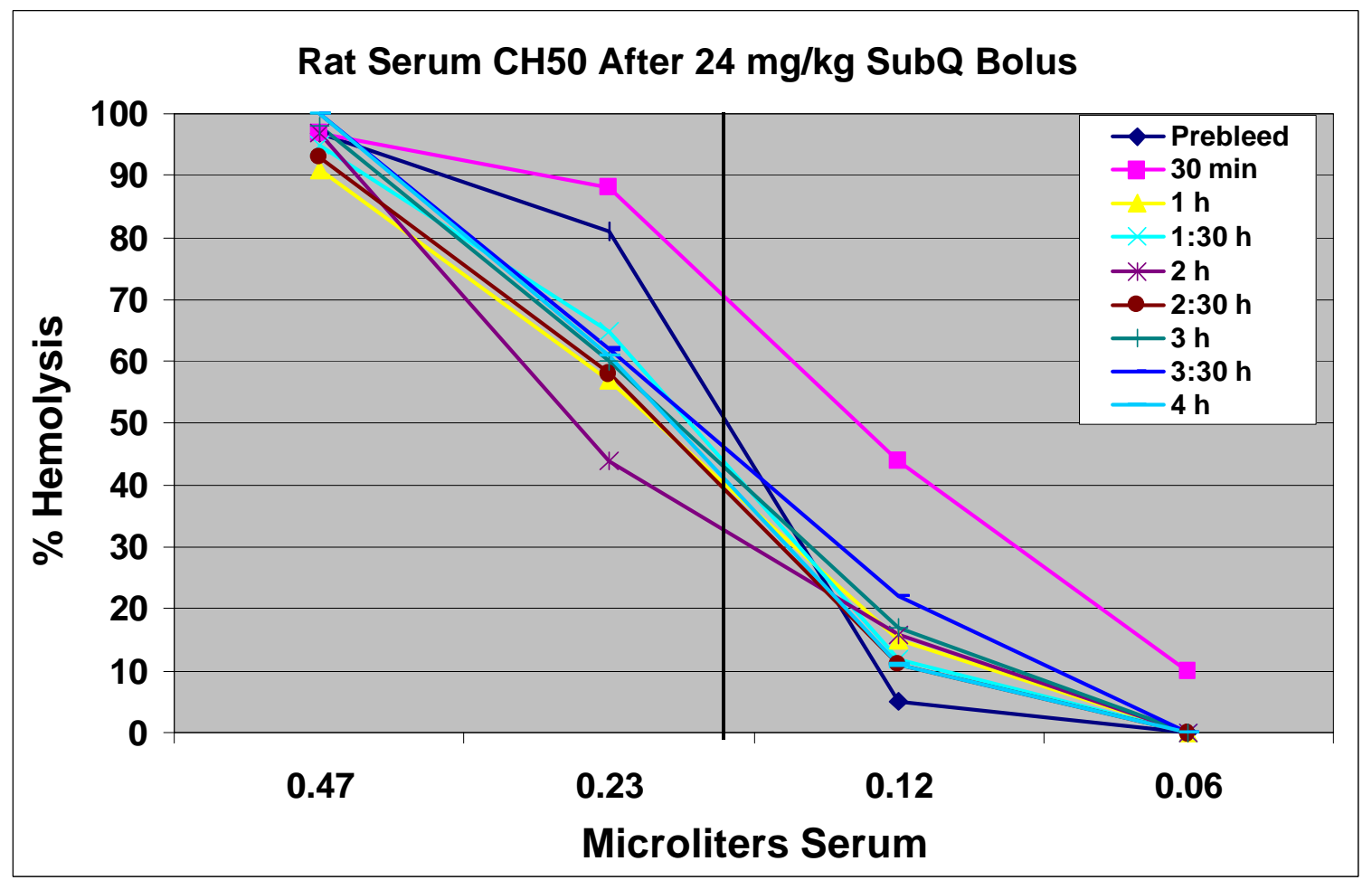


Figure 64. Baboon 479 CH50 following $9 \mathrm{mg} / \mathrm{kg}$ I.V. bolus. Serum samples were tested for complement activity by CH50 assay. CH50 activity was determined for 15 min, $30 \mathrm{~min}, 60 \mathrm{~min}$, and $120 \mathrm{~min}$. As can be seen, only a slight decrease in complement activity can be seen after 15 min. The verticle black line represents CH50 for pretreatment serum. 


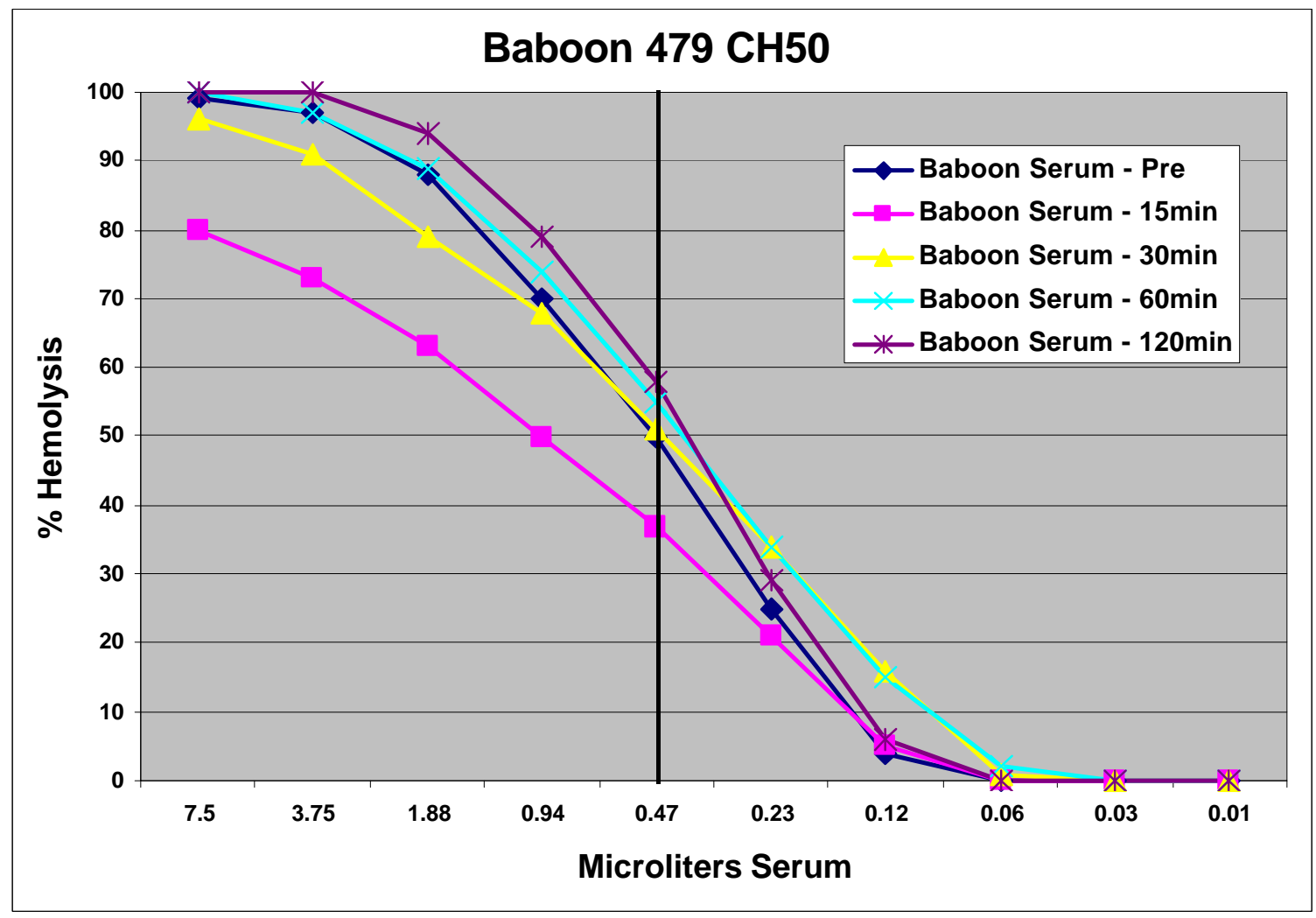


Figure 65. Rat serum CH50 following continuous $16 \mathrm{mg} / \mathrm{kg} / \mathrm{hr}$ via catheter. A rat was administered $\mathrm{rVCP}$ at $16 \mathrm{mg} / \mathrm{kg} / \mathrm{hr}$ by continual infussion and the serum $\mathrm{CH} 50$ was evaluated at 15 minute intervals for one hour. The verticle black line represents CH50 for pre-treatment serum. 


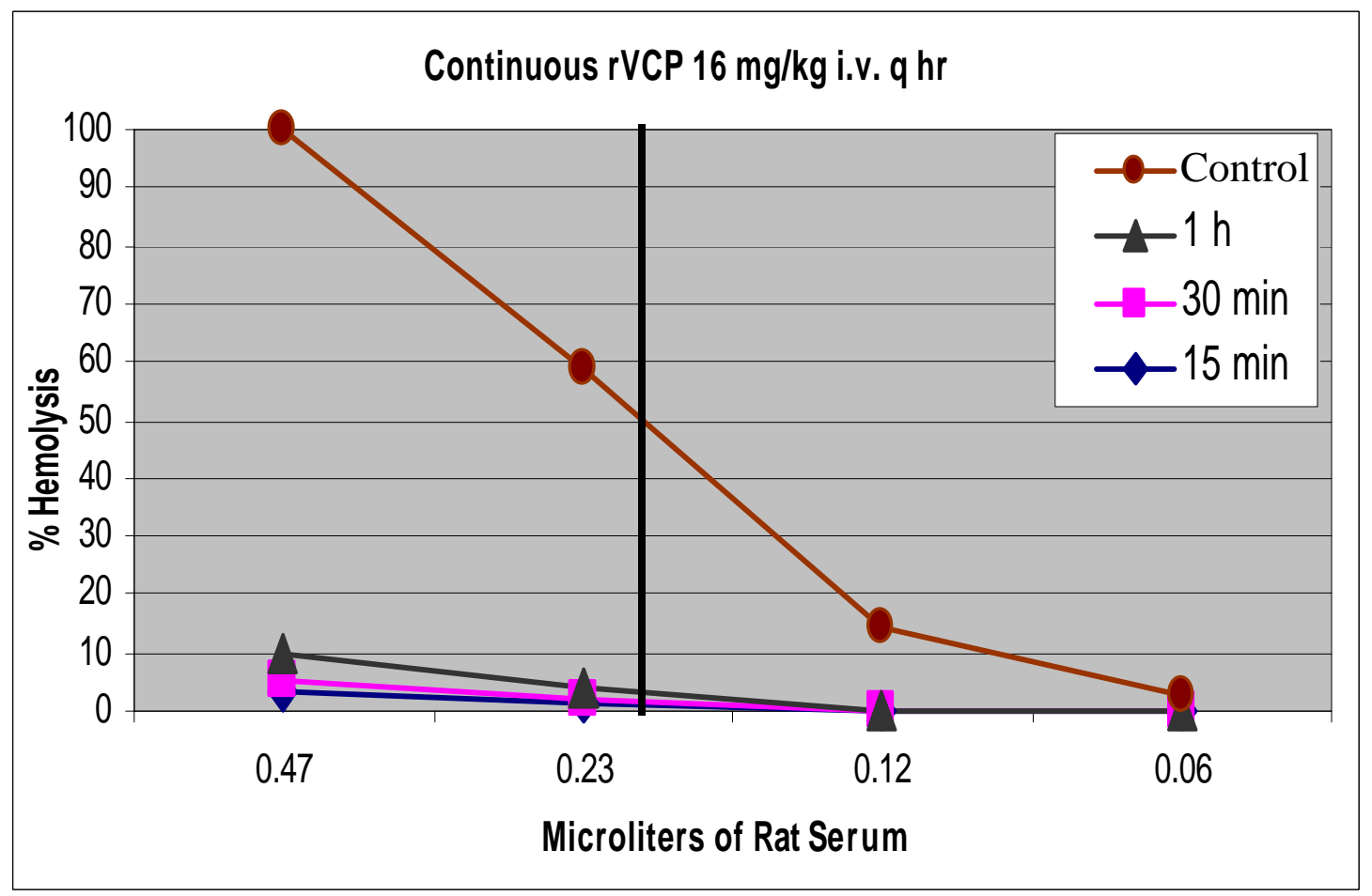


Figure 66. Rat serum CH50 following $6 \mathrm{mg} / \mathrm{kg}$ I.V. $+3 \mathrm{mg} / \mathrm{kg} / \mathrm{hr}$ via pump. Following the bolus injection, serum CH50 was assayed at 15 min and every hour for 6 hours. As can be seen, the initial $\mathrm{CH} 50$ was reduced to near 0 , but slowly returned toward normal levels. The verticle black line represents $\mathrm{CH} 50$ for pre-treatment serum. 


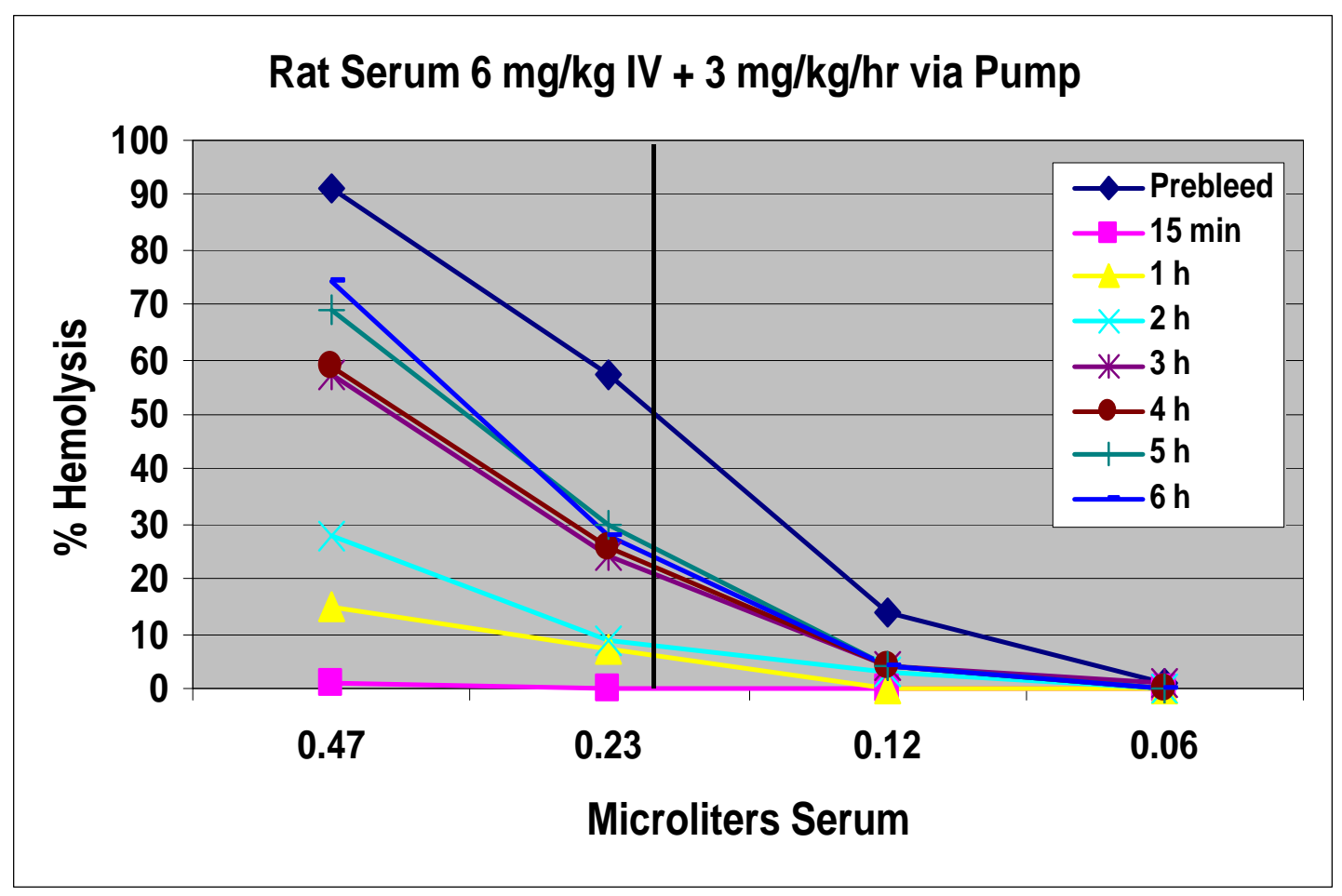


Figure 67. Rat Serum CH50 following $6 \mathrm{mg} / \mathrm{kg}$ I.V. $+6 \mathrm{mg} / \mathrm{kg} / \mathrm{hr}$ via pump. Following the bolus injection, serum CH50 was assayed at 15 min and every hour for 3 hours. The initial $\mathrm{CH} 50$ was reduced to near 0, but quickly returned toward normal levels. This was attributed to clogging of the injection line from using too high a concentration of rVCP. The verticle black line represents $\mathrm{CH} 50$ for pre-treatment serum. 


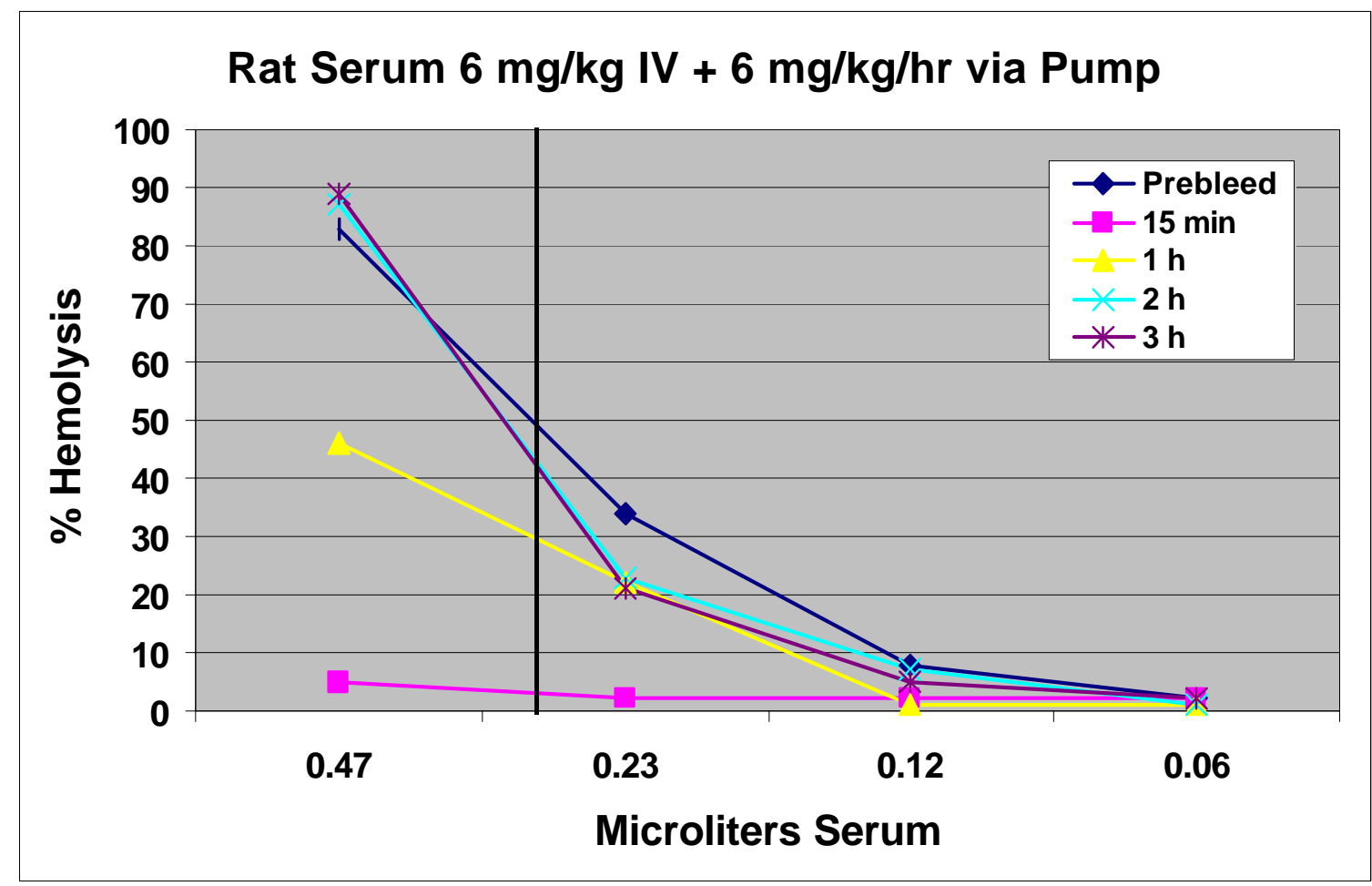


Figure 68. Rat Serum CH50 following $6 \mathrm{mg} / \mathrm{kg} \mathrm{I.V.}+6 \mathrm{mg} / \mathrm{kg} / \mathrm{hr}$ via catheter. Following the bolus injection, serum $\mathrm{CH} 50$ was assayed at $15 \mathrm{~min}$ and at 6 hours. The CH50 was reduced to near 0 , and remained that way for at least 6 hours. The verticle black line represents $\mathrm{CH} 50$ for pre-treatment serum. 


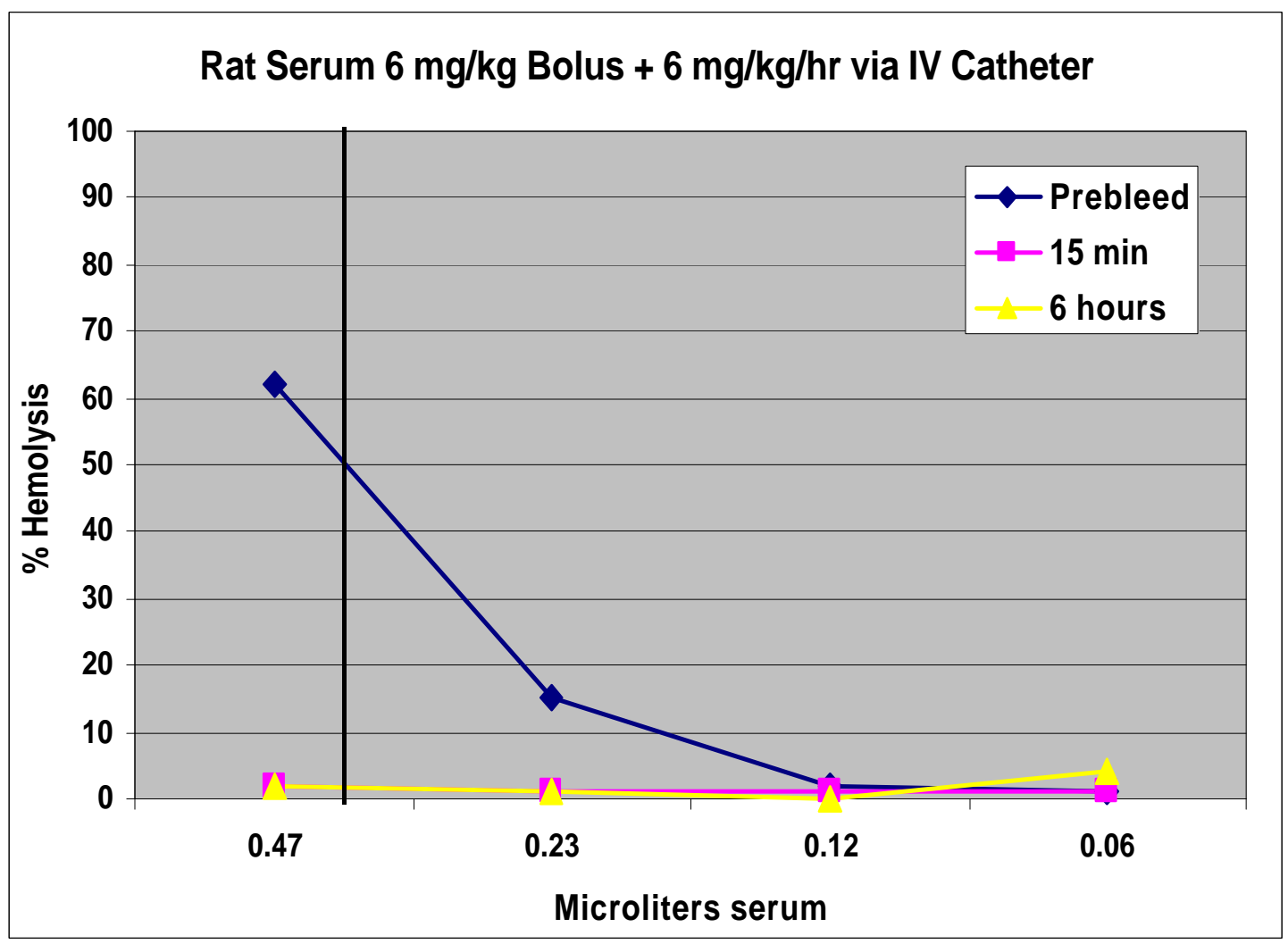




\title{
CURRICULUM VITAE
}

\author{
Scott Alan Smith \\ 1432 S. $3^{\text {rd }}$ St. Apt. 34, Louisville, Kentucky 40208 \\ (502) 634-2780, sasmit85@ hotmail.com
}

\section{Education}

Graduate: $\quad$ University of Louisville, School of Medicine, Department of Microbiology \& Immunology

Louisville, KY

Ph.D. in Microbiol. \& Immunol., 2002 GPA: 3.93

Undergraduate: $\quad$ California University of Pennsylvania

California, PA

B.S. in Biology, Pre-medicine, $1997 \quad$ GPA: 3.91

Dean's List: Fall 1995, 1996, 1997; Spring 1996, 1997, 1998; Summer 1996, 1997

Community College of Allegheny County (CCAC)

Pittsburgh, PA

A.S. in General Studies, $1995 \quad$ GPA: 3.88

Dean's List: Fall 1993, 1994; Spring 1994, 1995; Summer 1994, 1995

\section{Positions Held}

Ph.D. Candidate, University of Louisville, School of Medicine, Department of Microbiology \& Immunology, Louisville, KY, 2000-2002

Graduate Teaching Assistant, Department of Microbiology \& Immunology,

University of Louisville, School of Medicine, Louisville, KY, 1999-2001

Research Assistant, Department of Virology, University of Cape Town, Cape Town, South Africa, May 2001-July 2001

\section{Professional Services}

Health Sciences Center-Graduate Student Association Representative for

Department of Microbiology \& Immunology, University of Louisville, School of Medicine, 1999-2000 
Microbiology and Immunology Student Organization (MISO) Representative, Department of Microbiology and Immunology, University of Louisville, School of Medicine, Louisville, KY, 2000-2002

\section{Professional Societies}

International Society for Leukocyte Biology, 1999-2000

Honors and Awards

- National Dean's List, 1994-1995

- Phi Theta Kappa International Honors Society, 1994

- Beta Beta Beta National Honorary Biological Society, 1997

- Academic Excellence in the Biological Sciences Award, 1998

- University of Louisville School of Medicine Student Travel Award, 1999

- University of Louisville Graduate School Travel Award, 1999

- University of Louisville Graduate Student Council Travel Award, 1999

- First place winner of Research! Louisville Graduate Student Division, 2000

- Center for Genetics and Molecular Medicine (CGeMM) fellowship, 2001

\section{Bibliography}

\section{Peer-Reviewed Research Articles:}

Smith S.A., N.P. Mullin, J. Parkinson, S.N. Shchelkunov, A.V. Totmenin, V.N. Loparev, R. Srisatjaluk, D.N. Reynolds, K.L. Keeling, D.E. Justus, P.N. Barlow, and G.J. Kotwal. Conserved surface-exposed K/R-X-K/R motifs and net positive charge on poxvirus complement control proteins serve as putative heparin binding sites and contribute to inhibition of molecular interactions with human endothelial cells: a novel mechanism for evasion of host defense. J. Virol. Jun. 74(12):5659-66, 2000 [Cover of J. Virol. Oct. 74(20), 2000].

Murthy K.H., Smith S.A., Ganesh V.K., Judge K.W., Mullins N., Barlow P.N., Ogata C.M., and Kotwal G.J. Crystal structure of a complement control protein that regulates both pathways of complement activation and binds heparan sulfate proteoglycans. Cell. Jan. 26, 104:301-11, 2001.

Hicks R.R., Keeling K.L., Yang M., Smith S.A., and Kotwal G.J. Vaccinia virus complement control protein enhances functional recovery following traumatic brain injury. J. Neurotrauma, Jun. 19(6):705-14, 2002.

Smith S.A., Krishnasamy G., Murthy K.H., Cooper A., Bromek K., Barlow P.N., and Kotwal G.J. Vaccinia virus complement control protein is monomeric, and retains structural and functional integrity after exposure to adverse conditions. Biochim. Biophys. Acta., Jul. 29;1598(1-2):55-64, 2002. 
Anderson J.B., Smith S.A., and Kotwal G.J. Vaccinia virus complement control protein inhibits hyperacute xenorejection. Transplant Proc., Jun. 34(4):1083-5, 2002.

Anderson J.B., Smith S.A., Wijk R.V., Chien S., and Kotwal G.J. Vaccinia virus complement control protein inhibits hyperacute xenorejection in a guinea pig-to-rat heterotopic cervical cardiac xenograft model by blocking both xenoantibody binding and complement pathway activation. Transpl. Immunol., In Press, 2002.

Anderson J.B., Smith S.A., Wijk R.V., Chien S., and Kotwal G.J. Vaccinia virus complement control protein inhibits hyperacute xenorejection in a mouse-tosensitized rat heterotopic heart xenograft model by blocking both xenoantibody binding. Transplant Proc., In Press, 2002.

Smith S.A., Arjunwadkar S., Krishnasamy G., Judge K., Murthy K.H., Pugh D.R., and Kotwal G.J. Mapping of regions within the vaccinia virus complement control protein involved in binding to key complement components and heparin using surface plasmon resonance. Submitted to Biochim. Biophys. Acta., 2002.

Billings B.B., Smith S.A., Lahiri D., and Kotwal G.J. Vaccinia virus encodes a neurovirulent virokine that acts as an enhancer of viral replication (EVR) in vivo in brain: Implications for a safe smallpox and a potential HIV vaccine. Submitted to Nature Neuroscience, 2002.

\section{Peer-Reviewed Review Articles:}

Smith S.A., and Kotwal G.J. Virokines: novel immunomodulatory agents. Exp. Opin. Biol. Ther., 1(3):343-357, 2001.

Smith S.A., and Kotwal G.J. Vaccinia virus. In: Encyclopedia of Molecular Medicine. John Wiley \& Sons Inc., Vol. 5, pp. 3324-3330, 2001.

Smith S.A., and Kotwal G.J. Immune response to poxvirus infections in various animals. Crit. Rev. Microbiol., 28(3):149-186, 2002.

\section{Abstracts:}

Smith S.A., N.P. Mullin, J. Parkinson, S.N. Shchelkunov, A.V. Totmenin, V.N. Loparev, R. Srisatjaluk, D.N. Reynolds, K.L. Keeling, D.E. Justus, P.N. Barlow, and G.J. Kotwal. Surface-exposed K/R-X-K/R sites and overall positive charge on poxviral complement control proteins contribute to heparin binding and to additional functional features. Presented at the Society for Leukocyte Biology $15^{\text {th }}$ International Congress, Cambridge, United Kingdom, September 22-26, 1999 abstract \#LB15-52.

Keeling K.L., Hicks R.R., Yang M.Y., Smith S.A., Kotwal G.J. Role of complement in traumatic head injury. The $6^{\text {th }}$ Annual Kentucky Spinal Cord and Head Injury Research Symposium, Lexington, KY, June 12-14, 2000. Program and Abstracts. 
Smith S.A., N.P. Mullin, R. Srisatjaluk, D.E. Justus, P.N. Barlow, and G.J. Kotwal. Surface-exposed K/R-X-K/R sites and overall positive charge on poxviral complement control proteins contribute to heparin binding and to additional functional features. Presented at the University of Louisville Student Research Day, Louisville, KY, November 15, 1999. Research! Louisville 1999 Poster Abstracts, abstract \#GR21.

Keeling K.L., Hicks R.R., Yang M.Y., Smith S.A., Reynolds D.N., Kotwal G.J. Evaluation of the vaccinia virus complement control protein in modulation of inflammation in a rat head injury model. The XIIIth International Poxvirus-Iridovirus Symposium, Montpellier, France, September 2-6, 2000. Program and Abstracts, p.117, abstract \#P67.

Kotwal G.J., Smith S.A., F. Al Mohanna, R. Purpar, R. Srisatjaluk, D.E. Justus, N.P. Mullin, J. Parkinson, and P.N. Barlow. Heparin binding activity (due to putative $\mathrm{K} / \mathrm{R}-\mathrm{X}-\mathrm{K} / \mathrm{R}$ sites) of vaccinia virus complement control protein confers numerous functions with tremendous implications in viral evasion. The XVIIIth International Complement Workshop, Salt Lake City, Utah, July 23-27, 2000. Program and Abstracts, p.76, abstract \#223.

Smith S.A., Murthy K.H., Barlow P.N., and Kotwal G.J. The 3-D crystal structure analysis of vaccinia virus complement control protein reveals a heat stable structure with distinct binding sites for complement components and heparan sulfate proteoglycans. Presented at the University of Louisville Student Research Day, Louisville, KY, October 30, 2000. Research! Louisville 2000 Poster Abstracts, abstract \#GR23 (First place).

Smith S.A., Murthy K.H., Barlow P.N., and Kotwal G.J. The 3-D crystal structure of vaccinia virus complement control protein reveals a stable structure with distinct binding sites for complement components and heparan sulfate proteoglycans. Presented at the $32^{\text {nd }}$ Annual Midwest Student Biomedical Research Forum, Omaha, Nebraska, February 16 and 17, 2001. Program and Abstracts, p.26, abstract \#P25.

Smith S.A., and Kotwal G.J. Vaccinia virus complement control protein is functionally stable under adverse physical conditions due to structural features: structure-function relationships of a novel viral immunomodulator with therapeutic potential. Presented at the $32^{\text {nd }}$ Annual Midwest Student Biomedical Research Forum, Omaha, Nebraska, February 16 and 17, 2001. Program and Abstracts, abstract \#O45.

Hicks R.R., Keeling K.L., Yang M.Y., Kozawa S., Smith S.A., and Kotwal G.J. Vaccinia virus complement control protein attenuates cognitive deficits following traumatic brain injury in rats. The $11^{\text {th }}$ International Congress of Immunology, Stockholm, Sweden, July 22-27, 2001. Scand. J. Immunol. 2001 Jul-Aug; 54 (S1): 110. 